CAMBRIDGE

TECHNICAL

SERIES 


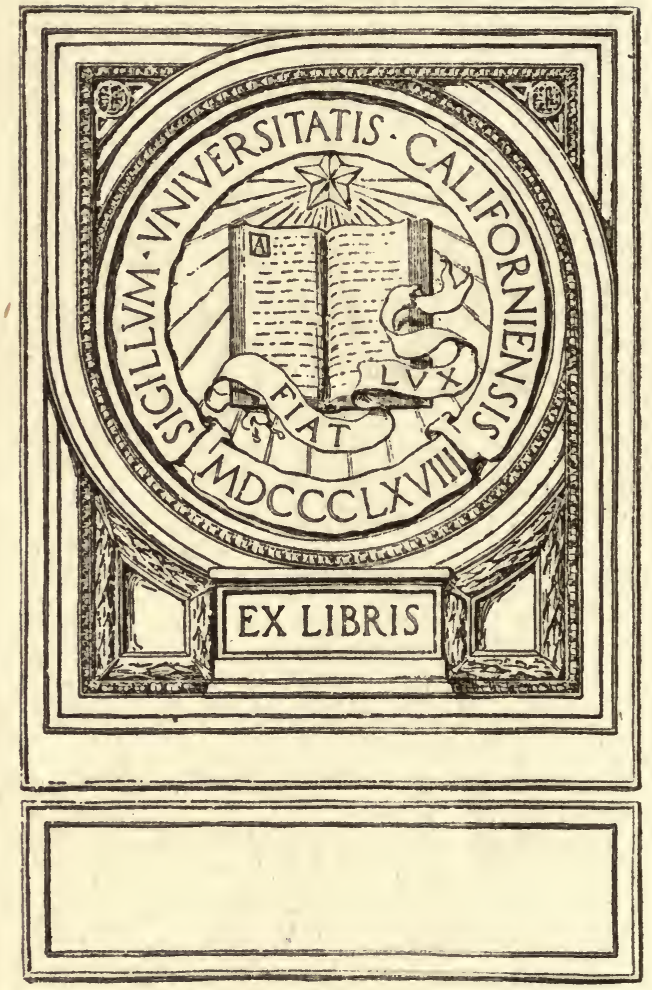



. 
The Cambridge Technical Series General Editor: P. Abbott, B.A.

\section{DOMESTIC SCIENCE}




\section{CAMBRIDGE UNIVERSITY PRESS}

C. F. CLAY, MaNager

Zlonton: FETTER LANE, E.C.

gedinburgh: 100 PRINCES STREET

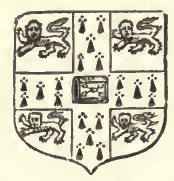

few Gork: G. P. PUTNAM'S SONS

bombay ano calcutta: MACMILLAN AND CO., ILTD.

Taronto: J.LM. DENT AND SONS, LTD.

Tokng: THE MARUZEN-KABUSHIKI-KAISHA 


\section{DOMESTIC SCIENCE}

BY

\section{CHARLES W. HALE, A.K.C.}

Science Master at the Sloane School, Chelsea;

Late Lecturer and Demonstrator in Chemistry at the

South-Western Polytechnic;

Examiner in Domestic Science to the National; Gloucester, Leicester and Sheffield Domestic Training Centres

PART I

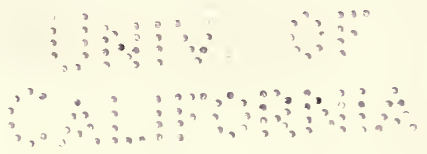

\section{Cambridge :}

at the University Press

I 9 I 5 
in Part II. Special attention has been paid to those portions of the subject which experience has shown are usually imperfectly grasped-perhaps on account of the difficulty of providing the student with ready examples of domestic application - and the theoretical side of the subject, e.g. the ideas of "energy" and of "molecular action", is treated more fully than is usual in works of similar character.

Parts I and II will provide sufficient material for a course of at least two years for pupils in secondary schools, and will be found to cover the requirements of many examinations, such as the Oxford Senior Local and those held under the auspices of the Board of Education at the various Domestic Training Centres.

The author's thanks are due to Messrs J. and A. Churchill for permission to reproduce Figs. 20, 27, 79, and 95, and to Messrs Macmillan and Co. for Figs. 21 and 87. For many valuable criticisms and useful suggestions, received from the General Editor of the series while the book was in the press, the writer tenders his grateful thanks.

\section{CHARLES W. HALE.}

Chelsea,

March, 1915.

Note.-The London County Council accepts no responsibility for the author's opinions and conclusions. 


\section{CONTENTS}

CHAP.

PAGE

I. Measurement of Length . . . 1

II. Measurement of Area and Volume . . 21

III. Measurement of Weight _ . . . 43

IV. The Relation between Weight and Volume 63

V. The Constitution of Matter . . . 70

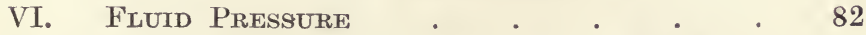

VII. Centre of Gravity . $\quad$ - . $\quad$. 108

VIII. Work, Power, and Energy. The Nature OF HEAT . . . . . . 118

IX. Temperature. Thermometers . . . 135

X. Heat as a Quantity - . . $\quad 159$

XI. The Propagation of Heat . • . 182

XII. Heat and Energy. The Kinetic Theory • 200

XIII. Air. Its Nature And Chinf Constituents 206

XIV. Minor Constituents of AIR . . 223

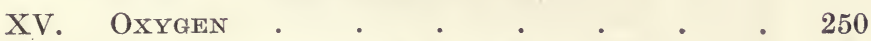

XVI. Physical Properties of Water . . 264

XVII. The Chemical Relations of Water . 280

XVIII. Chemical Classification • • • • 306

Answers to Questions involving calculations 317

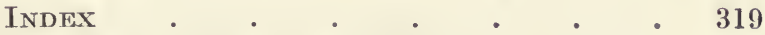




\section{LIST OF ILLUSTRATIONS}

FIG.

PAGE

1. Scale showing inches and centimetres . . 5

2. Measurement of length with scale of inches . 6

3. Method of graduation and eross section of scale . 9

4 Measurement of curved lines . . . . 14

5. Inside and outside calipers . . . . $\quad$. 17

6. Method of using simple vernier . . . . 19 .

7. Comparison of square foot and square decimetre 22

8. Areas of simple rectilinear figures . . . 26

9. Area of irregular figure by use of squared paper 29

10. Relation of area of circle to square of radius . 32

11. Comparison of eubic inch with cubic centimetre 34

12. Volume of rectangular parallelopiped . . 35

13. Volume of rectangular slab . . . . $\quad$. 36

14. Triangular and hexagonal prisms . . . 37

15. Right circular cylinder . . . . . $\quad$. 37

16. Graduated vessels for measurement of capacity 38

17. Method of reading graduated vessels . . 40

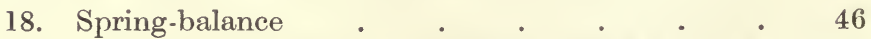

19. Apparatus for illustration of Principle of Moments 48

20. Simple chemical balance . . . . . 51

21. Method of holding pipette . . . . 58

22. Method of using burette . $\quad$. $\quad$. 59

23. Steelyard . . . . . . . . 60

24. Density flasks . . $\quad . \quad$. $\quad . \quad$. 67

25. Apparatus for determination of fluid pressure at different depths in a liquid . . . 84 
FIG.

PAGE

26. Simple hydraulic press . . . . . $\quad . \quad 87$

27. Method of weighing a solid in a liquid . $\quad 89$

28. Hydrometer . $\quad$ • $\quad$. $\quad$. $\quad$ - 92

29. Apparatus for illustrating variations of barometric pressure . . .6 .67

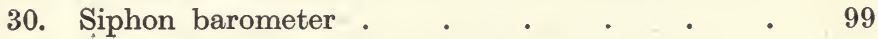

31. Apparatus for demonstrating Boyle's Law . 102

32. Centre of gravity of a plane figure . . . 109

33. Positions of varying stability of a thin lath . 112

34. Expansion of a bar of metal . . . . 124

35. Gravesande's ball and ring . . . . 126

36. Unequal expansions of different liquids . . 127

37. Expansion of a gas . . . . . . 128

38. $\mathrm{S}$ and $\mathrm{X}$ pieces for strengthening brickwork - 132

39. Method of softening capillary tubing • . 137

40. First stage in making thermometer . . 138

41. Method of filling thermometer . . . 139

42. Thermometer after sealing . . . . 140

43. Determination of freezing-point on thermometer 141

44. Checking boiling-point on thermometer . . 143

45. Simple mercury gauge for increasing pressure on boiling liquid. . . . . 144

46. Boiling water under reduced pressure . $\quad 145$

47. Clinical thermometer . . . . . 149

48. Index of minimum thermometer . . . 150

49. Index of maximum thermometer . . . 151

50. Six's maximum and minimum thermometer . 152

51. Simple form of calorimeter . $\quad$. $\quad$. 169

52. Determination of latent heat of steam . . 170

53. Contraction in volume of ice on melting . 174

54. Determination of specific gravity of air . $\quad 176$

55. Ingenhaus's conductivity apparatus . . 184

56. Water a poor conductor of heat . . . 185 
FIG.

57. Thermos flask $\quad . \quad$. $\quad$. $\quad$. $\quad$. $\quad$. 186

58. Convection currents in a liquid . . . 187

59. Convection currents in a gas . . . . $\quad$. 189

60. Effect of nature of surface on heat absorption . 195

61. Candle burning with limited air-supply . . 207

62. Candle burning with increased air-supply . 207

63. Burning candle in closed vessel . . . 209

64. Deflagrating-spoon . . . . . . 210

65. Burning phosphorus in closed vessel . . 211

66. Holder for stick phosphorus . . . . 213

67. Composition of air by slow oxidation of phosphorus 214

68. Composition of air by rusting of iron . . 215

69. Preparation of phosphorus pentoxide . . 216

70. Heating copper in exhausted vessel . . 219

71. Composition of air by passage over heated copper 220

72. Increase of weight due to combustion . . 228

73. Illustration of Principle of Indestructibility of Matter . . . . . . 230

74. Aspiration of air through lime-water . . 232

75. Curve showing hygrometric state of atmosphere 236

76. Wet and dry bulb hygrometer . . . 237

77. Chemical hygrometer . . . . . 240

78. Oxygen a waste product of plant life . . 243

79. Determination of volume of gases dissolved in water . . . . . 245

80. Lavoisier's experiment on the composition of air 251

81. Laboratory preparation of oxygen . . . 254

82. Burning coal-gas in oxygen . . . . 256

83. Burning steel in oxygen . . . . . 259

84. Evaporation to dryness in glass vessel . . 265

85. Distillation of tap-water . . . . . 267

86. Distillation of brine with Liebig condenser - 268

87. Still with worm-condenser . . . . 270 
FIG.

PAGE

88. Alum crystals . . . . . . 272

89. Potassium chlorate crystals . . . . 274

90. Method of folding filter-paper . . . . 275

91. Action of steam on heated iron . . . 282

92. Electrolysis of acidulated water . . . 284

93. Laboratory preparation of hydrogen . . 285

94. Filling balloon with hydrogen . . . 287

95. Water produced by combustion of hydrogen . 288

96. Explosion of mixture of hydrogen and air . 290

97. Anomalous expansion of water . . . 292

98. Kipp's generator . • . . • . 295

99. Composition of water by passage of hydrogen over heated copper oxide $\quad . \quad 296$

100. U-tube eudiometer . $\quad$. $\quad$ • $\quad$. 299

101. U-tube eudiometer with vapour-jacket . . 301

102. Potassium sulphate and potassium bisulphate crystals . . . . . . . 314 


\section{CHAPTER I}

\section{MEASUREMENT OF LENGTH}

1. Exact measurement is the foundation of science, hence our first concern will be to consider how measurements are usually stated, and to follow this by the description of some simple measuring instruments and how to use them.

In speaking of any measurement we use two words -a name and a number. The name is that of some chosen measurement with which the other measurement is compared, and is termed the " unit" measure. The number tells us how many times the actual measurement in question contains the unit measure. Let us suppose the measurement we wish to make is that of the length of the edge of a table. We may use as our unit of length that of a page of this book. By comparing this length in some suitable manner with that of the table-edge, we may find that the table is eight times as long as the book. We express this by the statement that the edge of the table is eight pagelengths long. The unit measure is here a "pagelength", and the number "eight" is the number of times the length of the table-edge contains the unit. Should another table of the same length be required, however, it would greatly surprise a cabinet-maker to be asked for a table eight page-lengths long. $\mathrm{He}$ would probably ask "How long is the page that you 
mean ? since all wovks do not have pages of the same length. On the other hand, if asked to make a table 6 feet long, he would not make any demur.

2. Units such as the foot, adopted by common consent, derive their utility from the greater convenience which arises from their general use. The chief units thus adopted are termed "Standard" units. In the British Isles the standard unit of length is the yard. At the Standards Office of the Board of Trade is kept a bar of bronze with a gold plug near each end. On each plug is engraved a fine transverse line. These lines are parallel to one another, and the legal yard is defined as "the distance between the centres of these lines when the bar is at a temperature of $62^{\circ} \mathrm{F} . "$ (The reason for mentioning the temperature will be understood after reading Chapter vIII.) Accurate copies of the standard are kept in all large towns, and by means of these the correctness of the yard-measures in actual use by tradesmen may be tested.

3. It usually happens that, when the length of an article is determined by measurement with a yardmeasure, its length is not found to be an exact number of yards. Hence yard-measures, tapes, carpenter's rules, and other instruments for measuring length, have marks upon them which divide the measure into a definite number of equal parts. The parts of the yard in common use-the foot and the inch-are called "submultiples" of the yard. The separate inches are generally subdivided into halves, quarters, eighths, or even smaller fractions of an inch. For measuring lengths which are much greater than the yard, units containing a given number of yards, such as the furlong and mile, are used. These are termed "multiples" of the standard unit. 
4. In dealing scientifically with any subject, simplicity is one of the most important points to keep in view. Hence for all scientific work, and in many countries for general use as well, a system of units has been adopted which does not involve much of the unnecessary labour, e.g. in memorising tables, and in making lengthy calculations, required by the British system. This is the "Metric " or "Decimal" system, introduced in $\mathbf{1 7 9 5}$ by the newly-born French Republic. It is based on the system of numeration in common use among civilised peoples, in which the relative positions of the figures in a number indicate their respective values. Thus 5555 expresses the sum of 5 thousands, 5 hundreds, 5 tens, and 5 ones, each 5 having a distinct value dependent on its place. Fractions of the unit are shown by placing a dot after the figure in the unit's place in the number, e.g. 5.5 denotes the sum of 5 units and 5 tenths of one unit. The standard of length chosen by the French Government was the Metre. This is defined as " the distance between the ends of a bar of platinum, preserved in the Court of Archives at Paris, when the bar is at the temperature of melting ice, i.e. $0^{\circ} \mathrm{C}$." The metre is longer than the yard, being $39 \cdot 37 \ldots$ inches. The multiples and submultiples of the metre are arranged in accordance with the decimal system so that each is exactly one-tenth of that next higher in value. Thus the table of metric measures of length is as follows:

10 millimetres $(\mathrm{mm})=$.1 centimetre.

10 centimetres $(\mathrm{cm}) \quad=$.1 decimetre.

10 decimetres $(\mathrm{dm}) \quad=$.1 METRE.

10 metres $(\mathrm{m}) \quad=$.1 decametre.

10 decametres $(\mathrm{Dm})=$.1 hectometre.

10 hectometres $(\mathrm{Hm})=$.1 kilometre ( $\mathrm{Km}$.$) .$ 
The prefixes milli-, centi-, and deci- are derived from the Latin, and are used to signify $\frac{1}{1000}, \frac{1}{100}, \frac{1}{10}$, respectively, while deca-, hecto-, and kilo- are from the Greek, and imply 10, 100, 1000.

5. Examination of the above table reveals two points in which it is superior to the British table. The name of one measure only has to be learnt and the names of the derived units are obtained by the addition of suitable prefixes to the name of the standard unit. One factor-10-is used throughout, whereas in the British table each new unit involves the memorising of a new factor. A further advantage of the Metric system which is not brought out by the consideration of a single table is that the same set of prefixes is used in other tables, thus further lessening the tax upon the memory. Other points of advantage will be pointed out as they arise in later work.

6. The value of a length expressed in terms of any one unit may readily be found in terms of any other, all that is necessary being the removal of the decimal point a certain number of places to the left or right, as the case may be, together with the addition, in some cases, of a number of zeros. Thus, 3.405 hectometres is evidently equivalent to 0.3405 kilometres or to 340,500 millimetres.

Exercises for Student.

1. Write down the number of centimetres in $(a) 48.07$. kilometres; (b) 6.0103 metres; (c) $0.17 \mathrm{~mm}$.

2. Write down the number of kilometres in $(a) 870.6$ millimetres ; (b) 4670800 metres.

3. 1 metre $=39 \cdot 37 \ldots$ inches. How many yards are there in 10 kilometres? How many kilometres are approximately equivalent to 62 miles? 
7. For making measurements of short lengths instruments called scales are used. These are flat pieces of steel or wood which have straight parallel edges, along which are marked graduations corresponding to certain of the smaller measures of length. Thus, in the scale of a part of which Fig. 1 is a diagram, the top edge is divided into inches by the long marks numbered 1, 2, 3, 4, and these are further divided into halves and tenths of inches by the shorter lines. Along the lower edge is a scale of centimetres subdivided into half-centimetres and millimetres. The

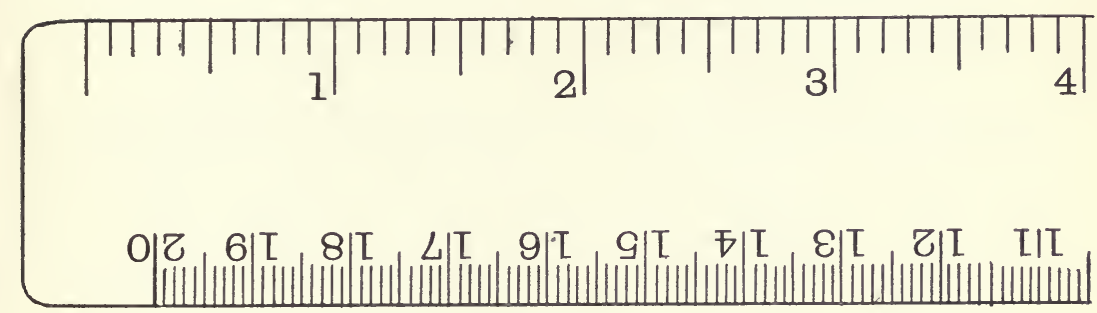

Fig. 1.

scale represented is made of wood, and it will be noticed that the last mark is not placed at the end of the scale. This is done to allow ready measurement to be made starting from the end mark, or "zero", even when the corners of the scale have been rounded off by rubbing while in use. With steel scales this precaution is less necessary, and the graduations on them frequently start at one end of the scale.

For measuring cloth, a tape-measure is generally employed. This cannot be used for exact measurements, as the material of which it is made readily stretches, or in damp weather, shrinks, and hence the 
true length of a piece of cloth is not that recorded by the tape. This is of special importance where material has to be cut out to fit, as in the case of window-blinds. The writer knows of a case in which a large number of windows were carefully measured by a skilled workman, and blinds made for them from his measurements. When these were delivered it was found, on fixing them up, that they were all about one inch too narrow. The blind-maker had used in measuring out the blind-stuff a 60 -inch tape which was too short by $1 \frac{3}{1}$ inches, while the workman had used a two-foot boxwood rule with a brass-bound edge which was, of course, a much more accurate instrument.

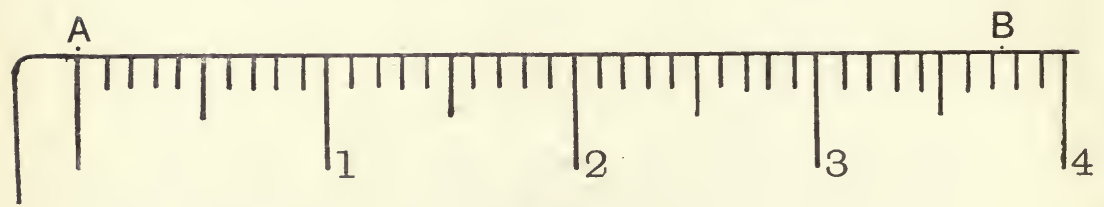

Fig. 2.

8. We will now show how to measure a short distance accurately by means of such a scale as that shown in Fig. 1.

Experiment 1. With a finely-pointed pencil make two small dots a few inches apart on a sheet of white paper. Letter these $\operatorname{dots} A$ and $B$.

Place the scale with the end mark on the inch scale at $A$ (see Fig. 2) and carefully adjust the edge of the scale (which should be thin) so that the point $B$ just touches the edge as in the figure. In judging whether $A$ is exactly opposite the last division of the scale, the eye should be placed vertically above the point $A$, and in reading off the measurement at $B$, 
the eye must also be vertically over point $B$. In the figure it will be seen that $B$ lies between the 3 rd and 4th inch marks, and also between the 7th and 8th marks (counting from the figure 3 ) which divide the 4 th inch into 10 equal parts. That is to say, the distance from $A$ to $B$ (or, as it is frequently termed, the distance $A B$ ) is between $3 \frac{7}{10}(3 \cdot 7)$ and $3 \frac{8}{10}(3 \cdot 8)$ inches. Let us now imagine the distance between the 7th and 8th subdivisions to be further divided into 10 equal parts. Each of these imaginary parts would be $\frac{1}{10}$ of $\frac{1}{10}$, or $\frac{1}{100}$ inch. From the 7 th mark to where the point $B$ touches the edge of the scale, we should estimate to be about four of our imaginary $\frac{1}{100}$ inch divisions. This amount must be added to $3 \frac{7}{10}$ inches. Hence the reading on the scale at $B$, and therefore the total distance from $A$ to $B$, is

$$
3 \frac{7}{10}+\frac{4}{100}=3 \frac{74}{100}(3 \cdot 74) \text { inches. }
$$

To verify this result a second determination of the distance from $A$ to $B$ should be made thus: place the scale so that the mark numbered " 1 " is at $A$, adjust till $B$ is on the edge of the scale, take the reading on the scale at $B$, and subtract one inch from this reading. A third measurement should also be made by placing the zero of the scale at $B$, and reading off the measurement at $A$.

Repeat the above measurements, using the edge of the scale divided into centimetres, estimating parts of a millimetre by the method given for estimating a fraction of an inch less than $\frac{1}{10}$ inch.

Tabulate your results, and find the mean (or average) value for the length $A B$ in inches by dividing the sum of the three measurements obtained by the number of measurements made. Similarly find the mean value of the length of $A B$ in centimetres. 
As an interesting exercise, calculate, from the mean results obtained, the number of centimetres in one inch by dividing the length of $A B$ in centimetres by its length in inches, and vice versa, the number of inches in one centimetre. The degree of correctriess of the results thus calculated will afford a measure of the accuracy with which the length $A B$ has been determined.

9. This simple experiment has been very fully described in order that the student may clearly understand how an experiment which is to yield satisfactory and accurate results is to be carried out. An account of what is done should be written out in a manuscript book, and the following is suggested as a suitable method of entry :

M. JoNES.

\section{Experiment 1.}

$18.7 . ' 14$.

Object. To measure the distance between two points in inches and in centimetres, and to find

(a) the number of centimetres in an inch;

(b) the number of inches in a centimetre.

Apparatus. A boxwood scale, of which the method of graduation and the cross section are shown in the diagram.

Method and Observations. Marked two small dots on the sheet of paper provided. Placed the end mark of the scale so that it coincided as closely as possible with one dot and adjusted till the other dot was just touching the edge of the scale. Took the readings on the scale opposite each dot.

Repeated this operation twice with the dots opposite different graduations on the scale. 
Similarly took readings, using the edge of the scale graduated in centimetres.

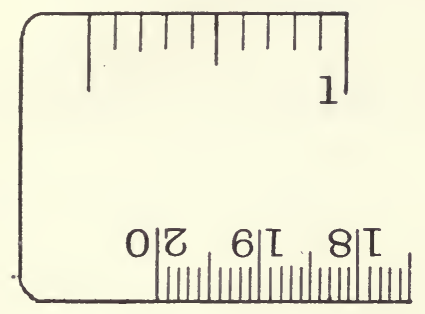

Method of Graduation.

Fig. 3.

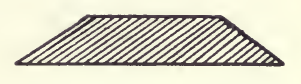

Cross section of scale.

Estimated hundredths of an inch and tenths of a millimetre by eye. The readings and results are given below.

$\begin{array}{cccc}\begin{array}{c}\text { Number of } \\ \text { measurement }\end{array} & \begin{array}{c}\text { Reading on } \\ \text { scale at } A\end{array} & \begin{array}{c}\text { Reading on } \\ \text { scale at } B\end{array} & \begin{array}{c}\text { Length of } \\ A B\end{array} \\ 1 & 0.00 \mathrm{in} . & 3.74 \mathrm{in} . & 3.74 \mathrm{in} . \\ 2 & 3.75 \mathrm{in} . & 0.00 \mathrm{in} . & 3.75 \mathrm{in} . \\ 3 & 1.00 \mathrm{in} . & 4.74 \mathrm{in} . & 3.74 \mathrm{in} . \\ 4 & 0.00 \mathrm{~cm} . & 9.50 \mathrm{~cm} . & 9.50 \mathrm{~cm} . \\ 5 & 9.53 \mathrm{~cm} . & 0.00 \mathrm{~cm} . & 9.53 \mathrm{~cm} . \\ 6 & 1.00 \mathrm{~cm} . & 10.51 \mathrm{~cm} . & 9.51 \mathrm{~cm} .\end{array}$

Results. Mean value of the length of $A B$ in inches

$$
=\frac{3 \cdot 74+3 \cdot 75+3 \cdot 74}{3}=3 \cdot 74 \ldots \text { inches. }
$$

Mean value of the length of $A B$ in centimetres

$$
=\frac{9 \cdot 50+9 \cdot 53+9 \cdot 51}{3}=9 \cdot 51 \ldots \text { centimetres. }
$$

Hence the number of centimetres in one inch

$$
=\frac{9 \cdot 51}{3 \cdot 74}=2 \cdot 54 \ldots
$$


and the number of inches in one centimetre

$$
=\frac{3 \cdot 74}{9 \cdot 51}=0 \cdot 394 \ldots
$$

10. The following points should be noted with respect to the suggested method of entry:

(a) The object of the experiment is placed first. To do an experiment merely because so directed in a text-book, or by a teacher, is of little service, and unless it is clearly seen with what object in view the given experiment is to be performed but little advantage is likely to accrue. To discover this object, it is best to read carefully through all the directions concerning the experiment, together with such parts of the immediate context as refer to matters directly connected with it.

(b) In describing apparatus, simple outline sketches are of the greatest value for two reasons :

(1) The drawing of a simple sketch frequently saves the writing of a long wordy description, while at the same time giving a clearer idea of the actual apparatus than could be afforded by any verbal account, however full.

(2) In attempting such sketches, the attention is focused more intently on the objects sketched than is the case when merely writing out a description, and this is of great service in fixing clearly in the mind the exact nature of the apparatus employed.

The student is advised to make simple outline diagrams rather than pretty pictures. The extra time and labour involved in making the latter may be more profitably made use of in perfecting the execution of the experiment itself. Many of the diagrams given in 
this book are of similar character to those suggested to be done by the student.

(c) The description of the experiment is best written in the past tense. On no account should the wording of the text-book be slavishly followed, and it is sometimes of service to read through the directions in the text-book and then to perform the experiment from memory, writing out an account of it entirely in one's own words. Further reference to the textbook should only be made in order to guard against the omission of any important portion of the work.

(d) Where a number of readings, or other observations of similar character, are made, they should be tabulated as affording a readier means of comparison of any variations noted.

(e) In stating numerical results, attention should always be paid to the accuracy with which measurements have been made, and no result should be so stated as to suggest that a greater accuracy has been attained than that of the least accurate measurement made. Thus, in the experiment as written out in paragraph 9 , the mean result of the measurement of $A B$ in inches is given as 3.74 inches, although the result of dividing the sum of the measurements by 3 gives $3 \cdot 7433 \ldots$ Here our measurements were made to the nearest hundredth of an inch, and hence our result must also be stated with that degree of accuracy. To say that the length of $A B$ is 3.743 inches would mean that it is not less than 3.7425 and less than 3.7435 inches, i.e. that the maximum error of measurement is $0.0005 \mathrm{in}$. and hence that the whole measurement is correct within $\frac{1}{2000}$ in. We cannot by the unaided eye measure length with such accuracy as this. That $A B$ is not less than 3.735 inches and less than 3.745 inches 
(i.e. that our measurement $3 \cdot 74$ inches is correct to $\frac{1}{200}$ or 0.005 inch) is a more probable statement.

Too much emphasis cannot be laid upon the importance of this point-that in all experiments involving measurement, every care should be taken in the statement of results that full regard is paid to the degree of accuracy of measurement of that quantity which is least susceptible of accurate estimation.

Where a measurement made is of less magnitude than that of the unit involved, it is usual to write its value in a decimal form, putting a zero in the units' place, e.g. a line less than an inch in length may be measured and its length said to be $0.86 \ldots$ inch.

11. The above method of entry may appear at first sight somewhat lengthy and troublesome, but experience shows that only by making certain that the work done can be described clearly and in full, is it feasible to train one's powers so that what has been done is retained and may be reproduced satisfactorily. Space and time may of course be saved in later work by making reference to that previously done. Thus, should a scale similar to that described above form part of the apparatus of a later experiment, all that it will be necessary to enter concerning it under the heading of apparatus will be: "A scale of inches and centimetres as described in Experiment 1". Similarly, the measurement of a straight line will not be again described fully, but may simply be said to be performed "as in Experiment 1".

12. As a further exercise in the use of a scale, the student should try

Experiment 2. Draw a line $C D 14.7 \mathrm{~cm}$. long, using the same scale as in Experiment 1. Measure 
the length of this line in inches, making at least three different determinations. Find the mean value of the length in inches, and then calculate

(a) the number of millimetres in one inch,

(b) the number of inches in one decimetre.

Compare the results obtained for $(a)$ and $(b)$ with those obtained in Experiment 1 for the number of centimetres in an inch and the number of inches in a centimetre.

13. Hitherto we have considered only the measurement of straight lines. Curved lines must be measured by special methods, such as those described in the following experiments.

Experiment 3. Draw a circle $B E F$ with a radius of 5 centimetres by means of a pair of compasses provided with a sharply pointed pencil. Draw any radius $A B$ (Fig. 4). By means of a millimetre scale set the points of a pair of dividers so that they are $2 \mathrm{~mm}$. apart. To test the accuracy of the setting, place one point of the dividers at $A$, and bring the other into contact with the line $A B$ at $C$. Keeping this latter point in position, remove the first from $A$ and rotate the dividers about $C$ until the point originally at $A$ is in contact with $A B$ at $D$. Continue this "stepping" along $A B$ until $B$ is reached. If the dividers have been correctly set, exactly 25 steps will be required to reach $B$. Should this not be the case, reset the dividers and once more step out a radius of the circle, repeating this test till satisfied with the accuracy of the setting. Next step out the circumference $B E F$ with the dividers as set, beginning at $B$. Note the number of steps required to go completely 
round the circumference. If we assume that each little bit of circumference measured by one step of the dividers is straight ${ }^{1}$, the number of steps multiplied by 2 is the number of millimetres in the circumference of the circle.

Repeat this measurement, having first set the points of the dividers $5 \mathrm{~mm}$. apart, and compare the length thus obtained with that previously found.

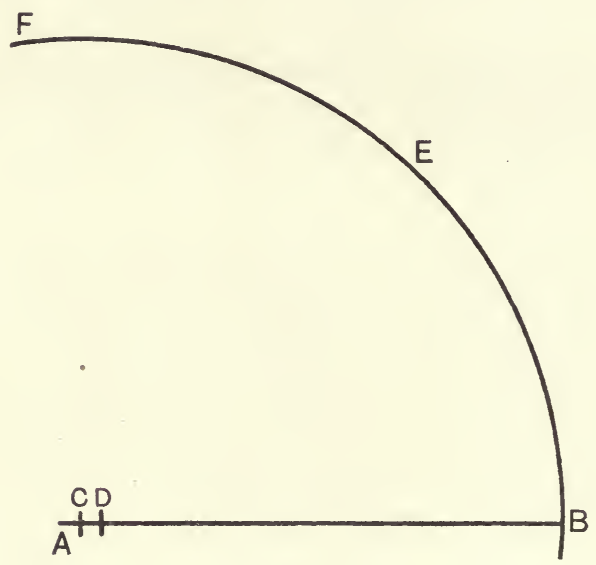

Fig. 4.

Exercise for Student.

How do you account for any difference noted ?

Draw two other circles with radii $4 \mathrm{~cm}$. and $6 \mathrm{~cm}$. respectively. Measure the lengths of their circumferences as above.

1 This assumption involves an error which is appreciable unless the length of a "step" is very small when compared with the length of the circumference, or, in other words, unless the number of steps required to go completely round the circumference is very large. 
As a check on the accuracy of your working, divide the length of the circumference obtained in each determination by the diameter of the circle.

Tabulate your measurements and values of the ratio $\frac{\text { circumference }}{\text { diameter }}$ and note that the latter all approximate to the same value. With very careful measurement, the average value will probably be about $3 \cdot 14 \ldots$ (to 5 places of decimals it is $3 \cdot 14159 \ldots$, but the method of measurement used is not sufficiently accurate to make it worth while to carry the result beyond two figures after the decimal point). This ratio is denoted by the Greek letter $\pi$ (pronounced "pie"). Hence it is usual to state that "the length of the circumference of a circle is $\pi$ times the length of its diameter", or that "the ratio of the length of the circumference of a circle to that of its diameter is $\pi$ ".

This method of measurement may be applied to curved lines other than circles, care being taken in every case that the length of "step" is made very short compared with the length of the curve to be measured.

Experiment 4. To measure the length of the circumference of a bottle, the following method may be used. Procure a supply of stout thread-or, better, of fine iron wire as used for binding a bunch of flowersand wind this five times around the circumference of the bottle so that the successive strands touch one another throughout their length, and the coil is parallel to the bottom of the bottle. The wire should be pulled sufficiently tight to lie in close contact with the exterior of the bottle. Bend the wire sharply at two points, one marking the beginning, the other the end, of the 
coil of five strands. Remove the wire, straighten it carefully by gentle stretching, and measure the distance between the two marks by bringing the wire in contact with the edge of a metre scale. The length read off is evidently five times that of the circumference of the bottle. Find the length of the circumference by division, and calculate the length of the diameter, making use of the result obtained in the previous experiment. Confirm the values obtained by performing two more determinations of the length of the circumference at the same part of the bottle, making coils of three and seven turns respectively.

The value found for the diameter may be readily checked by measuring it directly with a pair of calipers (see Fig. 5 B). Open the instrument so that the points of the jaws are separated by a distance slightly greater than the length of the diameter as found by calculation. Place the points on the circumference of the bottle in the region where the coil was applied and move the calipers to and fro on the surface, adjusting the distance between the points meanwhile so that they just slip over the outside of the bottle with the line between the points parallel to its base. Remove the. calipers, place the points of the jaws alongside the metre scale, and read off the distance between them. Make two further determinations, placing the jaws in each case at different parts of the circumference. Find the mean of the three values obtained and compare it with the mean of those already found.

In this experiment no allowance has been made for the thickness of the wire. Should this be considerable as compared with the diameter of the bottle, the length of the diameter as determined must be corrected by subtracting the thickness of the wire. 
Exercise for Student.

What corresponding correction must be made in the length of the circumference as found by experiment?

The above method, or simple modifications of it, may be used in finding the lengths of various parts of objects made up of curved surfaces, and for determining other lengths when the latter do not readily admit of direct measurement.

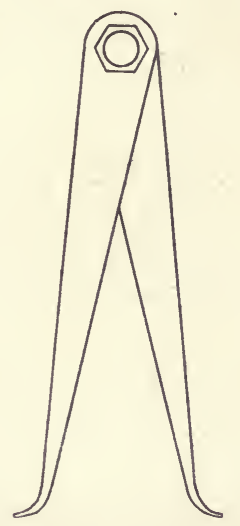

A

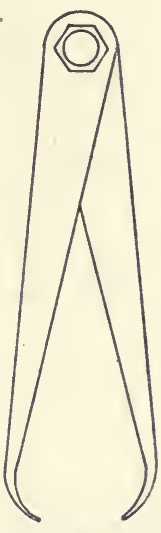

B

Fig. 5 .

Fig. $5 \mathrm{~A}$ shows the calipers arranged with the jaws crossed so that the points are directed outwards instead of inwards. Thus arranged, the instrument is of considerable service in determining the internal diameter of a vessel.

Experiment 5. Arrange the calipers as in Fig. 5 A. Place the points of the jaws just within the mouth of an ordinary tumbler, and adjust the distance between

H. D. S. 
them so that when the instrument is held by the hinge between the thumb and forefinger and twirled slightly; the points slide easily on the surface of the tumbler, yet still remain in contact with the glass. Remove the calipers and determine the distance between the points with a scale. Make two more measurements of the internal diameter of the tumbler at its mouth, arranging the jaws of the calipers along a different diameter in each case. Find the mean internal diameter from your results.

Next determine similarly the mean internal diameters $(a)$ midway between the top and bottom of the tumbler, $(b)$ at the bottom of the tumbler.

\section{Exercise for Student.}

Devise two distinct methods of finding the thickness of the glass of the tumbler by means of the calipers. Carry out both methods and compare the results obtained.

Greater accuracy of measurement may be attained by the use of calipers provided with a scale, and capable of more delicate adjustment by means of a screw-thread; for greater ease of reading, the scale is provided with a second small sliding-scale similar to that described in the next paragraph.

14. Some difficulty will probably have been experienced in estimating tenths of a millimetre by eye as suggested in Experiment 1. Where such accuracy in reading a finely divided scale is required, a small subsidiary scale, known as a "vernier", is used in order to relieve the strain upon the eyes involved in closely examining small scale-divisions. A simple form of vernier is illustrated in Fig. 6. $A B$ represents the vernier, which is attached to a scale showing centimetres and millimetres in such a way that it slides 
freely along the edge of the latter. It consists of a scale $0.9 \mathrm{~cm}$. long, divided into ten equal parts. Suppose we wish to know the reading on the large scale at a given point, such as $C$, to the nearest tenth of a millimetre. The zero mark of the vernier is adjusted so as to coincide with $C$ as in the figure. The reading is evidently between 18.1 and $18.2 \mathrm{~cm}$. To obtain a measurement to $0.01 \mathrm{~cm}$., examine the graduations of vernier and scale and note where one graduation mark on the vernier is as nearly as possible in the same straight line as one of the marks on the scale. In the figure this occurs at the 7 th vernier

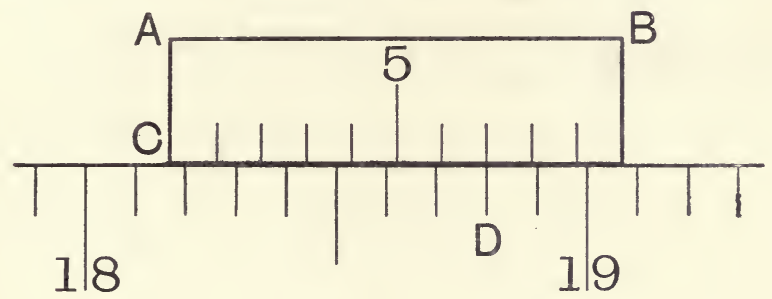

Fig. 6.

division at point $D$. This shows that $0.07 \mathrm{~cm}$. must be added to $18.1 \mathrm{~cm}$. to obtain the reading at $C$ to $0.01 \mathrm{~cm}$. This reading is consequently $18.17 \mathrm{~cm}$.

That this is the ease is rendered evident by the following considerations. Each vernier division is equal to $0.9 \mathrm{~mm}$., while each of the small divisions of the scale is $1.0 \mathrm{~mm}$. Hence the 6 th vernier division is $0.01 \mathrm{~cm}$. from the first scale-division to the left of $D$, the 5 th is $0.02 \mathrm{~cm}$. from the 2 nd scale-division to the left, and so on. Finally, the zero mark of the vernier at $C$ is $0.07 \mathrm{~cm}$. from the 7 th division on the scale to the left of $D$, i.e. from the division which shows $18.1 \mathrm{~cm}$., and 
therefore $0.07 \mathrm{~cm}$. must be added to $18.1 \mathrm{~cm}$. to obtain the reading at $C$ correct to $0.01 \mathrm{~cm}$.

The vernier described above is of the type known as "forward reading", i.e. the numbers of the divisions of the vernier and those of the scale both increase in value from left to right, and the reading of the vernier is taken in the same direction as that of the scale. A vernier $1 \cdot 1 \mathrm{~cm}$. long, with ten equal divisions as before, is said to be "backward-reading". In this case, the zero of the vernier scale is at the right-hand end, and the numbers of its divisions increase in value from right to left, so that when the zero is placed in the required position, the reading on the vernier is obtained by looking along the scale in the opposite direction to the usual. The latter type possesses the advantage of a more open scale, the slightly larger divisions upon it being more readily distinguished, and hence causing less eye-strain. This may be still further obviated by reading the vernier with the help of a lens of low power.

Many other forms of vernier are used in connection with various scientific instruments, but the consideration of their methods of construction and their uses is beyond the scope of our work. Practice in reading a simple vernier may be obtained by examination of those which may be found attached to the scales of an ordinary standard barometer.

\section{QUESTIONS}

1. The radius of a penny is $0 \cdot 6^{\prime \prime}$ and that of a 10 -centime piece is $1.5 \mathrm{~cm}$. By how many $\mathrm{mm}$. is the circumference of a penny longer than that of a 10-centime piece?

2. A 60 -inch tape-measure is actually $60 \frac{3}{4}$ inches long. What would be the error in the length of a cricket pitch, which should 
be $22 \mathrm{yds}$. long, if the pitch were actually laid out by means of such a tape-measure?

3. A tennis court is $78 \mathrm{ft}$. long and $36 \mathrm{ft}$. wide. How many metres does the marker travel in marking out the lines around the outer edge of the court? If the radius of the marking-wheel is $3 \frac{1}{2}$ in., how many times does the wheel revolve in whitening one long edge ?

4. The minute hand of a clock is $4^{\prime \prime}$ long and the hour hand $23_{4}^{\prime \prime}$. In how many hours will the tip of the minute hand have travelled a mile and how many times must the hour hand travel round the clock-face before its tip has covered the same distance?

5. An ebony ruler is $10^{\prime \prime}$ long and has a diameter of $1^{\prime \prime}$. How many feet of string must be wound around the ruler, with the coils just touching, to cover it with a layer of string from end to end, the diameter of the string being $0^{\circ} 1^{\prime \prime}$ ?

6. Write a clear description of the method you would adopt in determining the thickness of the material of a coffee-pot.

7. What is a "vernier"? How would you construct a vernier for use with a foot-rule divided into tenths of an inch, so as to obtain measurements to the nearest hundredth of an inch ?

\section{CHAPTER II}

\section{MEASUREMENT OF AREA AND VOLUME}

15. Before dealing with the units of area, and the methods used in measuring areas, we must know exactly what is meant by an area. The area of a figure is the size of the surface bounded by the line or lines which make up the sides of the figure. In this book we shall only deal with areas all points of which lie in the same plane, i.e. with areas marked out on a flat surface. Thus each square in Fig. 7 is a plane figure, as long as the page is kept flat, but if the page 
is bent in any way so as to distort either square, the latter ceases to be a plane figure.

16. In measuring small areas, the units generally adopted are the square inch and the square centimetre in the British and Decimal systems respectively. By a square inch (or square centimetre) is meant an area equal to that contained within a square the
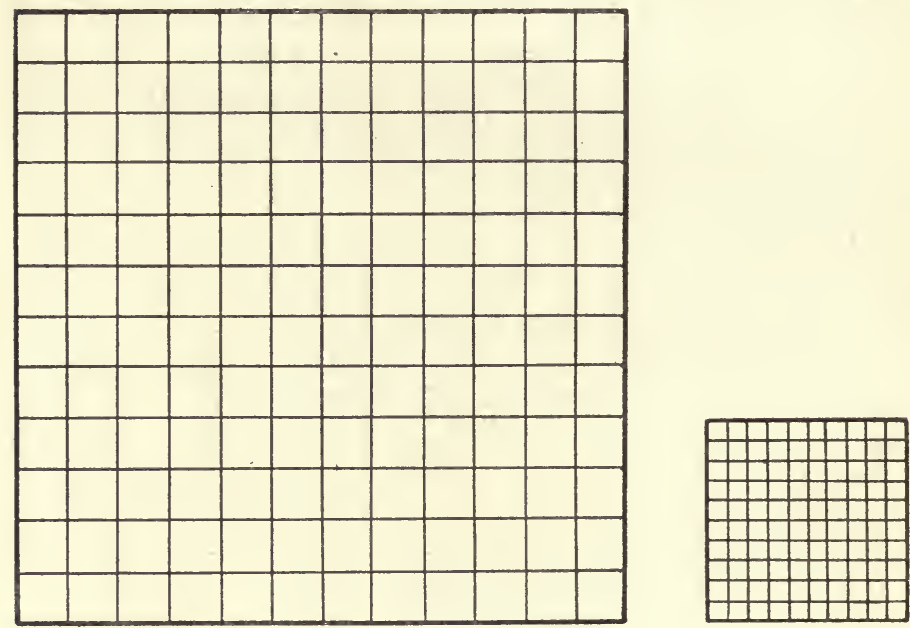

Fig. 7.

length of the side of which is one inch (or one centimetre). Since 1 foot $=12$ inches, and 1 decimetre $=$ 10 centimetres, it may readily be shown that 1 square foot, i.e. an area equal to that contained within a square the length of the side of which is 1 foot, contains 144 square inches, and 1 square decimetre is equal to 100 square centimetres. Thus Fig. 7 represents a square foot divided into square inches and a square decimetre divided into square centimetres, both being drawn to 
the same scale, and each square being $\frac{1}{25}$ its actual size.

The chief units of area in use in our country are given in the following table, together with their relations to one another and to the square inch.

$$
\begin{aligned}
& \text { Table of Measures of Areas. (British System.) } \\
& \begin{aligned}
144 \text { square inches (sq. in.) } & =1 \text { sq. foot. } \\
9 \text { sq. ft. (or } 1296 \text { sq. in.) } & =1 \text { sq. yard. } \\
4840 \text { sq. yd. (or } 6,272,640 \text { sq. in.) } & =1 \text { acre. } \\
640 \text { acres (or } 4,014,489,600 \text { sq. in.) } & =1 \text { sq. mile. }
\end{aligned}
\end{aligned}
$$

Let us compare this with the table of areas arranged on the decimal system, treating the square centimetre as the unit corresponding to the square inch, thus :

Table of Measures of Areas. (Decimal System.)

$$
\begin{aligned}
& 100 \mathrm{sq} . \mathrm{mm} . \quad \ldots \quad \ldots \quad \quad \ldots \quad \ldots=1 \mathrm{sq} . \mathrm{cm} \text {. } \\
& 100 \text { sq. cm. } \quad . \quad \quad \ldots \quad \ldots \quad \quad . . \quad=1 \text { sq. dm. } \\
& 100 \text { sq. dm. (or 10,000 sq. cm.) .. }=1 \text { sq. m. } \\
& 100 \text { sq. m. (or 1,000,000 sq. cm.) .. }=1 \text { sq. Dm. } \\
& 100 \text { sq. Dm. (or 100,000,000 sq. cm.) =1 sq. Hm. } \\
& 100 \text { sq. Hm. (or 10,000,000,000 sq. cm.) =1 sq. Km. }
\end{aligned}
$$

Note. In measuring land, the square decametre $(=100$ sq. metres $)$ is termed the "are", and 100 square decametres make 1 "hectare".

On examining the numbers expressing the ratios between successive units in each of the above tables, and also the numbers connecting each unit in the first table with the square inch and in the second table with the square centimetre, the overwhelming simplicity of the Decimal system as compared with our own will at once be seen. 
17. The method used to determine the area of a given figure depends to some extent upon the nature of the figure. If the figure is regular or symmetrical in outline a few simple measurements of certain lengths in it will usually afford sufficient data from which to obtain its area by easy calculation. The areas of certain well-known regular figures may be found by calculation as described below.

(a) Area of rectangle (oblong).

A rectangle is a four-sided rectilinear (straightlined) figure having all its angles right angles.

(The square is a special example of a rectangle in which all the sides are equal.)

The figure $A B C D$ in Fig. 8 is a rectangle. The side $A B$ represents $6.00 \mathrm{~cm}$., and the side $B C$ represents $4.00 \mathrm{~cm} .{ }^{1}$ The lines drawn on the figure divide it up into little squares, the sides of which represent $1.00 \mathrm{~cm}$. If we count the squares, we shall find that the total number in the figure is 24 . Hence the area (or quantity of surface enclosed within the rectangle) is 24.0 square centimetres and this number is readily obtained by multiplying 4 by 6 . From consideration of this and similar examples, we may deduce the following simple rule :

To find the area of a rectangle, measure the lengths of two adjacent sides, using the same unit of length for each. Multiply together the number of units of length in the one side by the number of units of length in the other side. The result will be the number of units of area in the rectangle, each unit being equal

1 The actual lengths of the lines in the figure as printed are one-half the values given above. All other lengths mentioned in paragraph 17 are reduced in like ratio. 
in area to the square which has as its side the unit of length employed.

Example. The adjacent sides of a rectangle are respectively $1.50 \mathrm{in}$. and $2.00 \mathrm{in}$. in length. The area of the rectangle is $(1.50 \times 2.00)$ sq. in. $=3.00$ square inches.

Note that we do not say that the area is $1.5 \mathrm{in} . \times$ $2 \cdot 0$ in. Numbers, but not lengths, may be multiplied together.

Such an expression as "Multiply together the lengths of two adjacent sides" is, however, commonly used, but must always be looked upon as simply an abbreviated method of writing "Multiply together the number of units of length in one side by the number of units of length in an adjacent side".

(b) Area of parallelogram.

A parallelogram is a four-sided rectilinear figure which has its opposite sides parallel.

The area of a parallelogram is found by multiplying the length of one side (such as $E F$, Fig. 8) by the length of the perpendicular drawn to it from any point on the opposite side.

Example. The area of the parallelogram $E F G H$ (Fig. 8$)$ is $(1.40 \times 4.60)$ sq. cm. $=6.44$ sq. $\mathrm{cm}$.

That this rule is correct may be readily seen if we cut off the triangular portion $F K G$, and place it at the opposite end of the parallelogram in the position shown by the dotted lines $E L, L H$, when the parallelogram becomes a rectangle $E F K L$ of which the area is, by our previous rule, equal to the product $E F \times F K$.

(c) Area of triangle.

A triangle is a three-sided rectilinear figure. The area of the triangle $M N O$ (Fig. 8) is evidently half the area of the parallelogram $M N O Q$, and is therefore 
half the product of the lengths of the side $N O$ and the perpendicular $M P$. Hence the rule :

To find the area of a triangle, draw from any of its angular points a straight line perpendicular to the

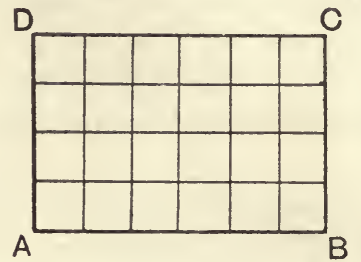

(1)

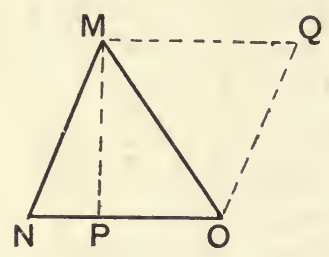

(3)

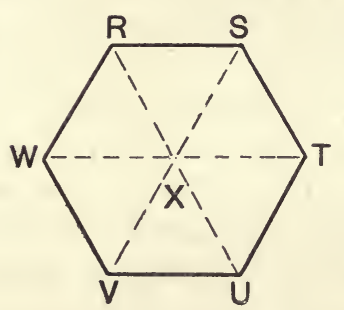

(5)

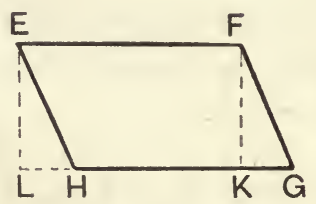

(2)

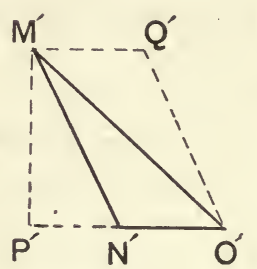

(4)

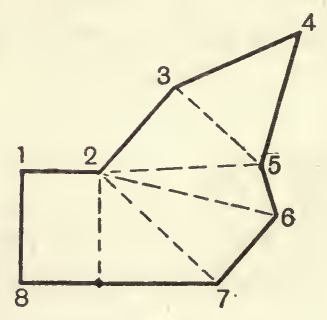

(6)

Fig. 8.

opposite side, or, if necessary, to the opposite side produced. Measure the length of this perpendicular and of the side to which it is perpendicular; half the product of these two lengths is the area of the triangle. 


\section{Example :}

(1) In the triangle $M N O$ (Fig. 8) the perpendicular $M P$ (usually called the "height") from $M$ (the " apex.") on NO represents $3.60 \mathrm{~cm}$., while NO (the "base") represents $4.00 \mathrm{~cm}$. Hence the area of the triangle is $\left(\frac{1}{2} \times 3.60 \times 4.00\right)$ sq. $\mathrm{cm} .=7.20 \mathrm{sq} . \mathrm{cm}$.

(2) In the triangle $M^{\prime} N^{\prime} O^{\prime}$ the height $M^{\prime} P^{\prime}$ represents $3.60 \mathrm{~cm}$. and the length of the base $N^{\prime} O^{\prime}$ represents $2 \cdot 20 \mathrm{~cm}$. Hence the area of the triangle $M^{\prime} N^{\prime} O^{\prime}$ is $\left(\frac{1}{2} \times 3.60 \times 2.20\right)$ sq. cm. $=3.96$ sq. $\mathrm{cm}$.

There are other special rules for finding by measurement and calculation the areas of certain other rectilinear figures, but, generally speaking, it is best to find the area of any such figure by dividing it into simpler figures, e.g. parallelograms or triangles, by diagonally drawn lines, finding separately the area of each of these component figures, and adding the areas thus obtained.

Thus the area of the regular hexagon RSTUVW (Fig. 8) is evidently six times that of the triangle $R S X$, and that of the irregular figure 12345678 is the sum of the areas of the smaller figures into which it is divided. by the dotted lines.

For practice in the above rules, the student should do

Experiment 6. Find by actual measurement and calculation the areas in square inches and in square centimetres of the regular hexagon and of the irregular figure shown in Fig. 8, (5) and (6).

From the results of the determinations made in each case, calculate the number of square centimetres in one square inch, and the number of square inches in one square centimetre.

18. In all cases already considered the sides of 
the figures have been straight lines. We will now describe a method of finding the area of any plane figure, whatever the nature of its sides.

Experimunt 7. Fig. 9 shows a rectangle 6.00 in. long and $4.00 \mathrm{in}$. broad, divided up by thick lines into squares each side of which is half an inch long, these being further divided by fainter lines into small squares of which the length of side is one-tenth $(0 \cdot 1)$ inch. Thus the whole rectangle is divided up into small squares the area of each of which is

$\left(\frac{1}{10} \times \frac{1}{10}\right)$ sq. in. $=\frac{1}{100}$ sq. in. $=0.01$ sq. in.

(To verify this, count the number of little squares in the square $A B C D$, which has sides $1 \mathrm{in.} \mathrm{long,} \mathrm{and}$ hence is $1 \mathrm{sq}$. in. in area.)

In the middle of the squared paper an irregular figure $M N O P Q R$ has been drawn. To find the area of this figure we count the number of little squares enclosed within the boundary of the figure. Since the boundary cuts many of the little squares into two or more parts, we must find in some way the areas of the little pieces of the figure thus cut off. This may be done by estimating by eye what fraction of a whole square each little piece is, and finding the sum of all the fractions. Adding this sum to the total number of complete small squares within the figure gives as a result the area of the figure, expressed in hundredths of a square inch. Dividing this result by 100 , the area in square inches is obtained.

It is easier in general, however, to count each fraction of a square larger than half a square as a whole square, and to ignore parts of less area than half a square. Should a square be cut exactly in half it may be counted as half a square. In doing this, we assume that the error 


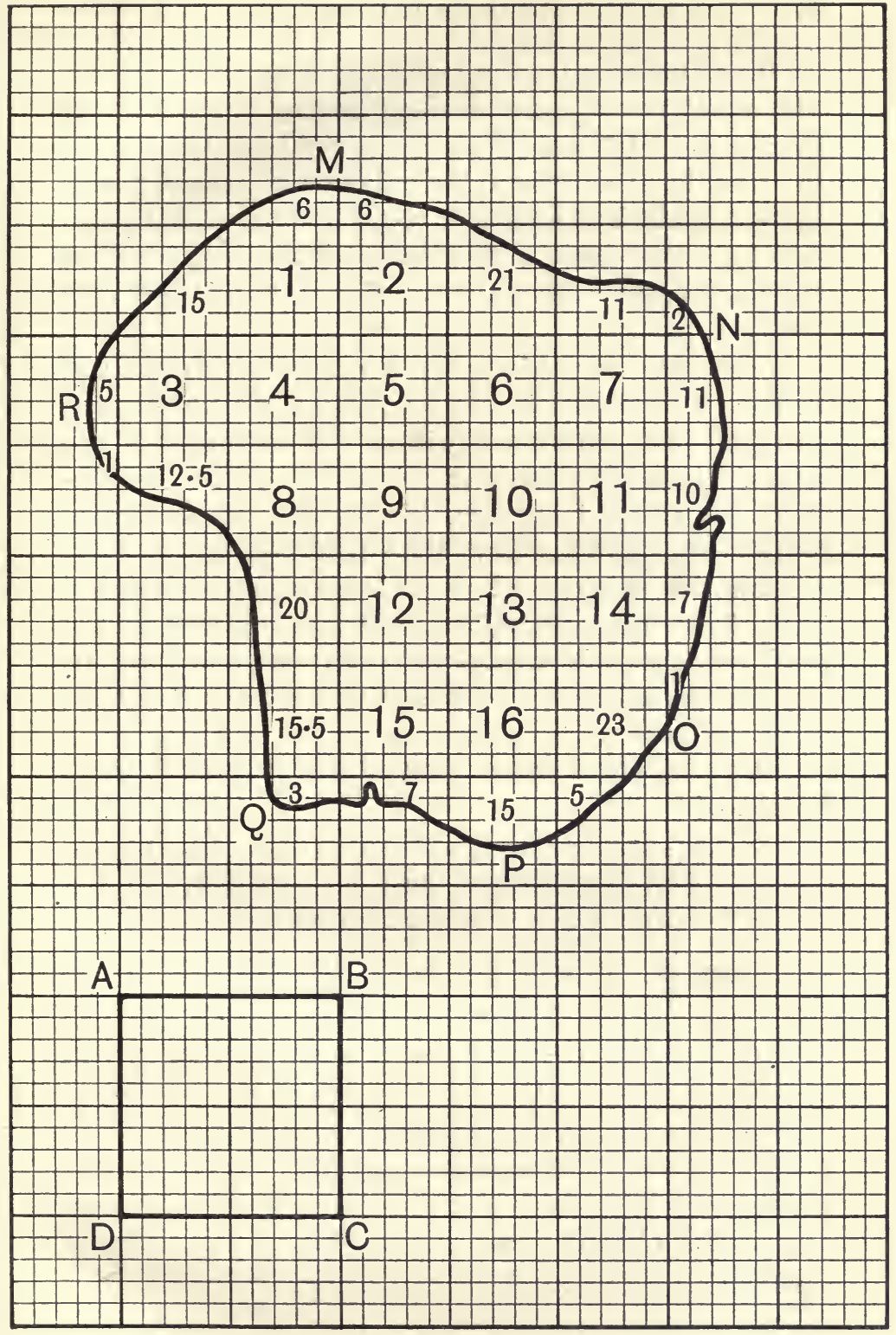

Fig. 9. 
made in counting the larger fractions as whole squares will be balanced by that made by leaving out of account the smaller fractions. This will not of course be always the case, but the error in the value of an area obtained by this method of counting, where the paper is divided into squares which are small, and the area itself is large compared with the area of 1 square, is not very great.

Example. In the irregular figure $M N O P Q R$ (Fig. 9) there are 16 complete large squares, each containing 25 small ones, contained within the boundary of the figure. These are numbered 1 to 16 (No. 8 is considered a complete square, because the small portion outside the figure is less than half a small square). Within the portions of large squares also contained in the figure are marked in smaller type the numbers of small squares each such portion contains, the fractions of small squares being estimated by the second method described above.

Thus the whole area of the figure $M N O P Q R$ is equal to that of

$$
\begin{aligned}
& (16 \times 25)+6+6+15+21+11+2+5+11 \\
& \quad+1+12 \cdot 5+10+20+7+15 \cdot 5+23 \\
& +1+3+7+15+5=597 \text { small squares. }
\end{aligned}
$$

Hence the area of the figure $M N O P Q R$ is

$$
(597 \times 0.01) \text { sq. in. }=5.97 \text { sq. in. }
$$

For practice in this method of estimating areas, perform the two simple experiments which follow.

ExPERIMENT 8. On a sheet of squared paper divided as described above, draw with a pair of compasses a circle as large as the paper will allow. Draw any radius of this circle, and on this radius describe a square. (See Fig. 10.) 
Find the area of the square in square inches by the method of measurement and calculation given. Find its area also by the method of counting the squares, and compare the results obtained.

(Note. Should there be any serious discrepancy between the results obtained by the two methods, the student is advised to test the accuracy of the division of the squared paper used by comparing the lengths of the sides of the large and small squares respectively with $0.5 \mathrm{in}$. and $0.1 \mathrm{in}$. as given by a wellmade scale of inches divided into tenths. A small error in these lengths will cause, when squared and multiplied by the number of squares within the figure, a very considerable error in the value obtained for the area.)

Experiment 9. Find the area in square inches of the circle described in Experiment 8 by counting the squares contained within its boundary. (It will be sufficient to count the squares in one quarter-circle or "quadrant", and multiply the number thus obtained by 4 .)

Find the ratio between the area of the circle and the area of the square on its radius, by dividing the former by the latter. Repeat the experiment by drawing on another sheet of paper a second circle slightly smaller than the first, finding its area as before, and determining the ratio of the area of this second circle to that of the square on its radius. It will be found that the value of this ratio, if the experiment be carefully done, is approximately $3 \cdot 14$. If the experiment be repeated many times, each time with a circle of different radius, it will be found that the ratio area of square on radius is always close to $3 \cdot 14$. 


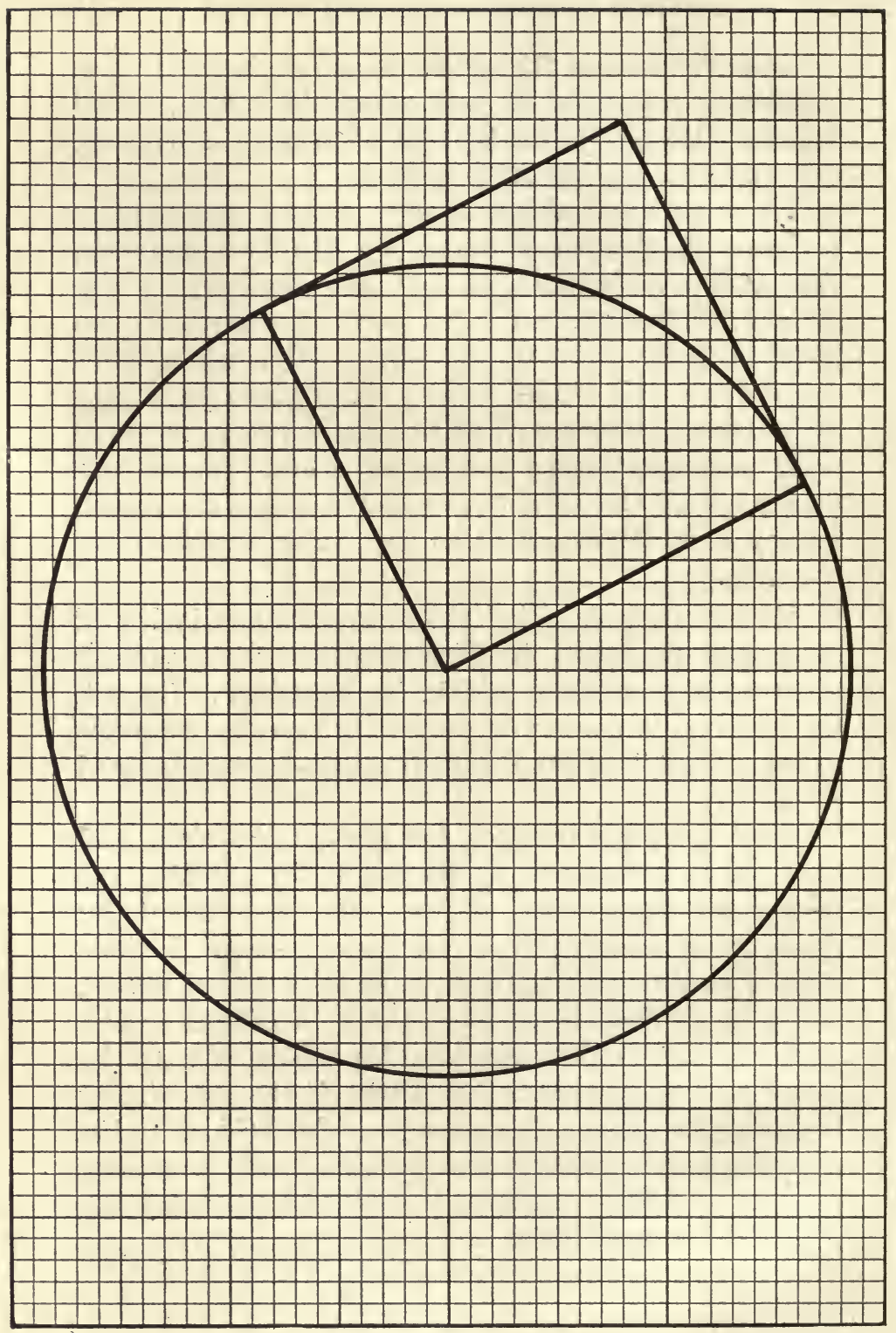

Fig. 10. 
This is the same ratio which we obtained in Experiment 3 , and called $\pi$.

From these results, the following rule for finding the area of a circle is obtained:

Measure the length of the radius, square this, i.e. multiply the number of units of length in the radius by itself, and multiply the result by $\pi$.

Example. The radius of a circle is 6.40 centimetres. Its area is consequently

$$
(6.40 \times 6.40 \times 3 \cdot 14) \mathrm{sq} . \mathrm{cm} .=129 \mathrm{sq} . \mathrm{cm} . \text { (nearly) } .
$$

Another method of finding the areas of irregular or curvilinear figures will be described in a later chapter dealing with the use of the balance.

Note. Instead of the squared paper divided into quarters and hundredths of a square inch as described above, paper similarly divided into square centimetres and square millimetres may be used. The areas may thus be obtained in square centimetres instead of in square inches. The use of such paper has, however, the great drawback that the counting of very small squares is a somewhat trying task, more especially to anyone suffering from defective vision.

19. The measurement of volume, i.e. the quantity of space occupied by a body, is carried out on principles similar to those governing the measurement of area. Thus the unit of volume is a cube, the length of each edge of which is unity. (A cube is a solid figure with six faces, each of which is a square.) The British units in common use are the cubic inch, cubic foot and cubic yard, while in the Metric System, the cubic centimetre and cubic metre are usually employed. In measuring a brick, its volume is generally stated in cubic inches or cubic centimetres; the volume of air 
in a room is usually expressed in cubic feet or cubic metres; the amount of material required to build a dam is given in cubic yards or cubic metres.

When dealing with the interior volume, or "capacity", as it is termed, of measuring vessels, an entirely different set of units is made use of in Britain. We ask for a pint or a quart of milk, not for a certain number of cubic inches of that fluid. No simple relation exists between the cubic inch and the pint, 1 pint containing $34 \cdot 66$... cubic inches. In the Metric System the unit of capacity is known as the "litre", and in this case there is a very simple relation between the ordinary
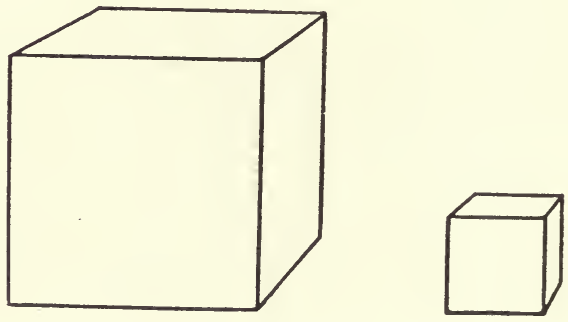

Fig. 11.

unit of volume and that of capacity, since 1 litre is exactly 1 cubic decimetre. It will be readily seen, therefore, that the measurement and comparison of volumes, when using the latter system, is much more easily carried out than is the case with the British System, thus emphasising what has already been written under this head when dealing with the measurements of length and area.

The comparative sizes of the cubic inch and the cubic centimetre are shown in Fig. 11. 
20. The volume of a rectangular slab may be found by performing

Experiment 10. From a bar of soap or a lump of plasticine cut out carefully a brick-shaped slab $6 \mathrm{~cm}$. long, $4 \mathrm{~cm}$. wide and $3 \mathrm{~cm}$. deep. (Or procure a small cardboard box of these or similar internal dimensions, pack it tightly with plasticine and then carefully remove the cardboard faces.) Mark off the consecutive centimetres along the four long edges of the slab and join the marks by parallel lines. (See Fig. 12.) Carefully

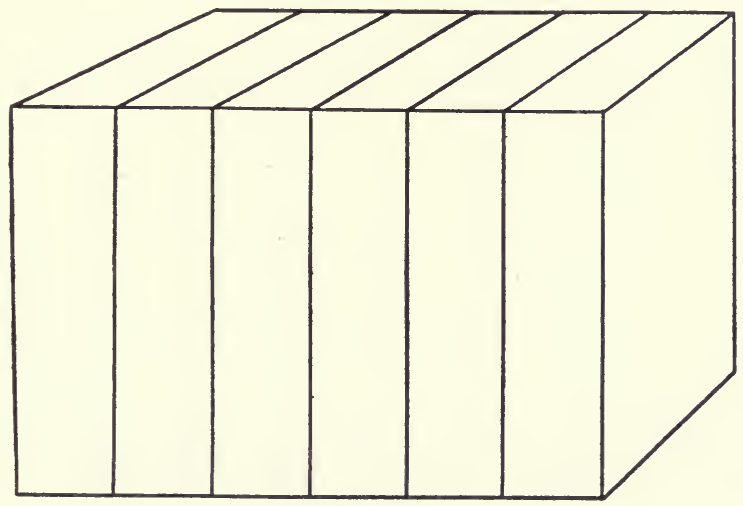

Fig. 12.

cut the slab along these lines. This is most readily done with a length of fine wire after the fashion of a grocer cutting cheese. The six smaller slabs thus formed have evidently the same total volume as that of the original slab. Mark out one face of one of the smaller slabs in square centimetres as shown in Fig. 13. Cut the slab along the lines thus obtained. It will be noted that each of the portions thus formed is a cube and that its edge is $1 \mathrm{~cm}$. long, i.e. it has a volume of 
1 cubic centimetre (c.c.). The whole of the last divided slab has a volume of 12 c.c. and, since the original slab consisted of six such smaller slabs, its volume must be $6 \times 12=72$ c.c.

By preparing other figures of similar shape; but of different sizes, and measuring their volumes by this method, it will be easy to see that the general rule for determining the volume of brick-shaped bodies

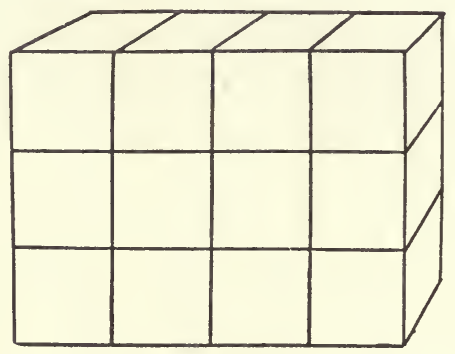

Fig. 13.

-or rectangular parallelopipeds, as they are termed in geometry-is as follows :

Find the area of one face of the body and multiply this by the length of any edge at right angles to that face.

21. The rules for finding by calculation the volumes of some other simple solid figures of regular shape may be summarised thus:

1. The Rectangular Prism. This is the name given to any solid figure with two end-faces at right angles to the remaining rectangular faces, which may number three or more. Fig. 14 gives representations of a triangular and a hexagonal rectangular prism. A brick is another familiar example. To find the volume of any such figure, determine the area of either end-face 
-usually termed the "base "-and multiply it by the length of the edge of any one rectangular face which is perpendicular to the base. This latter length is called the "height" of the prism.

2. The Cylinder. This is really a rectangular prism with a circular base. Its volume is also found by multiplying the area of the circular base by the height of the cylinder. In this case the height is found by measuring the length of any straight line on the surface of the cylinder perpendicular to the base.
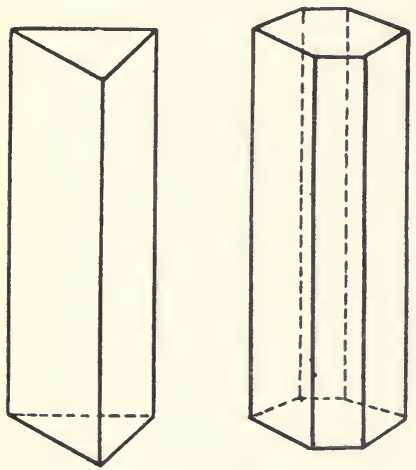

Fig. 14.

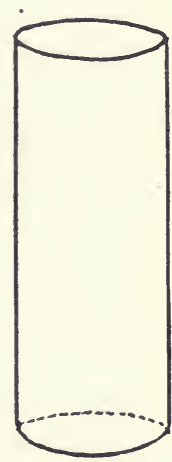

Fig. 15.

3. The sphere. The volume of a sphere may be found by multiplying the cube of its radius by $\frac{4}{3} \pi$.

4. The Pyramid. Multiply the area of the base by the height, i.e. the length of the perpendicular from the apex upon the plane containing the base, and divide the product by 3 .

5. The Cone. This is simply a pyramid with a circular base, and the rule given above applies.

To facilitate the solution of problems dealing with the volumes of the above solids, the following formulae may be of service : 


$$
\begin{array}{rlrl}
\text { Volume of } & \text { Prism } & =b h ; \\
, & & \text { Cylinder } & =\pi r^{2} h ; \\
, & & \text { Sphere } & =\frac{4}{3} \pi r^{3} ; \\
, & & \text { Pyramid } & =\frac{1}{3} b h ; \\
, & & \text { Cone } & =\frac{1}{3} \pi r^{2} h .
\end{array}
$$

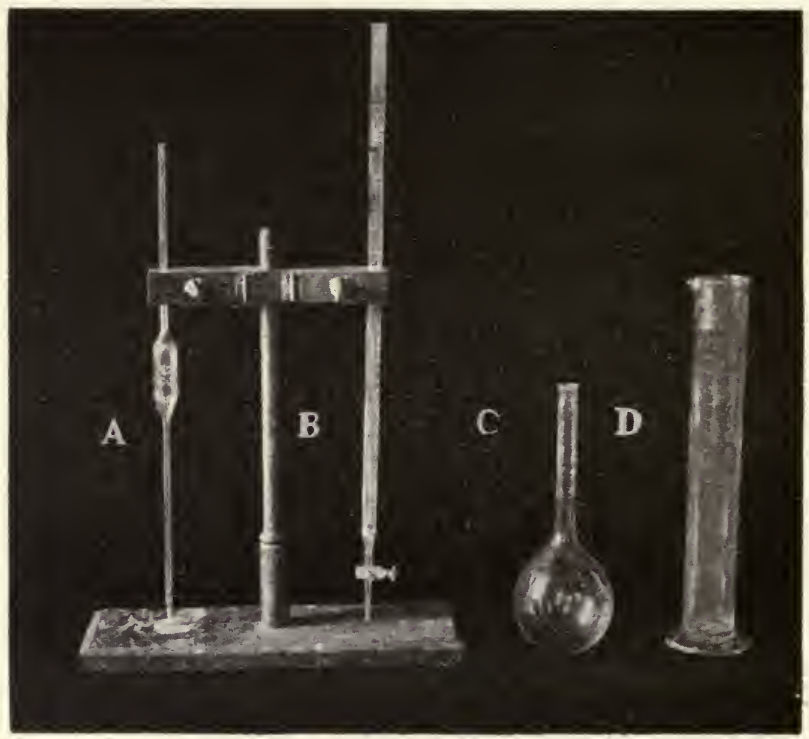

Fig. 16.

(The significations of the symbols $b, h, r$ may be readily gathered from the preceding statements concerning the volume of each figure.)

The volumes of solids of irregular shape, such as a lump of coal or a glass stopper, are usually very difficult to determine by direct measurement. Other methods of finding the volumes of such bodies are given later. 
22. The volumes of liquid bodies are generally determined by using measuring vessels of suitable size, which are graduated in different ways, according to the purpose for which they are intended.

$A$ in Fig. 16 represents the "pipette". This is used when a given volume, consisting of a whole number of c.c., such as 5, 10, 20 or 50 c.c., is required to be delivered into another vessel. One graduation mark only is needed, situated on the upper part of the stem. The pipette, when filled with water to this mark, will deliver the volume of liquid marked on its bulb. This should be allowed to run out freely and the small quantity left in the jet should be permitted to remain there, as this is allowed for when graduating the instrument.

By means of $B$, the "burette", volumes involving fractional portions of a cubic centimetre may be measured, the graduations on the body of the burette usually showing cubic centimetres and tenths of a cubic centimetre. Burettes are constructed to deliver total volumes of $10,25,50$ or 100 c.c.

The measuring flask, $C$, holds the number of c.c. marked on it when filled to the mark on its neck. Flasks of varying sizes are in common use, those most frequently met with being constructed to hold 50, 100, $200,250,500$ or 1000 c.c. Some flasks will be noticed as having two marks on the neck. When filled to the lower mark the flask contains the volume marked on its body. When filled to the upper mark the volume marked on it may be poured from it. The volume between the two marks is the allowance made for the drops of liquid which remain clinging to the interior of the flask when it is used to pour out a quantity of liquid. 
The measuring cylinder, $D$, may be of different capacities, varying in general from 10 to 1000 c.c. It is graduated in cubic centimetres, the actual value of each graduation depending on the size of the vessel.

Similar measuring vessels may be obtained, graduated in accordance with the requirements of the British units of capacity, but of these we will only consider one, the pint measure, graduated to show submultiples of a pint. Here again we find that the British arrangement is complex, since the pint is graduated in ounces. One pint of pure water at $62^{\circ} \mathrm{F}$. weighs 20 ounces and the measure is marked to show these and fractions, such as $\frac{1}{4}$ and $\frac{1}{2}$, of one ounce.

23. Experiments involving the use of the vessels mentioned will be described in later chapters and for the present we will be content to show how the large measuring cylinders may be of service in some cases in determining the volumes of irregular solids.

Experiment 11. Partly fill a litre cylinder with water. Read carefully from the scale on the outside of the vessel the number of c.c. of water in the cylinder. It is well to examine the scale before doing this in order to note the exact value of each scale-division. Thus, in Fig. 17, there are 10 divisions between the 500 and 600 c.c. marks. Each of these represents therefore 10 c.c. The level of the liquid surface is curved upward near the inner wall of

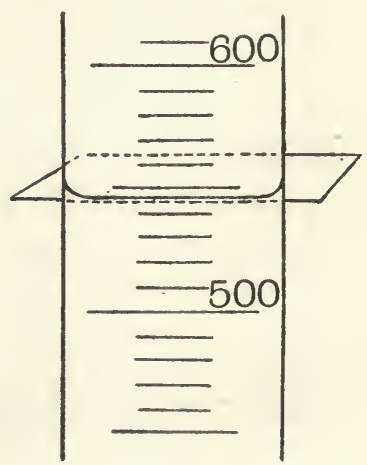

Fig. 17. the containing vessel and the 
reading must be taken at the lowest point of this curved surface, which is called the "meniscus". Should the lower edge of the meniscus not be sharply defined, a white card should be held obliquely behind the cylinder, with its lower edge just below the meniscus, when the latter will stand out sharply (see Fig. 17). The reading as shown in the figure is 547 c.c., the units figure being estimated by imagining each scaledivision to be further divided into 10 equal parts, each representing l c.c.

Drop a lump of coal or other irregularly-shaped solid object - which must be made of material impervious to, denser than, and not acted upon by, waterinto the cylinder. The level of the water will rise and, after ascertaining that no air-bubbles are clinging to the immersed body, take the reading at the new level. Suppose this to be 682 c.c. It is evident that the solid has displaced a bulk of water equal to its own and that its volume is consequently $(682-547)=135$ c.c.

In the case of solids acted upon by water, a similar method of finding the volume may be employed, substituting for water a liquid which does not affect the solid.

Experiment 12. Find the volume of a large lump of crystallised copper sulphate (bluestone or blue vitriol) by the method of the previous experiment, using paraffin oil instead of water.

A further method of determining indirectly the volume of a solid body is described in Chapter vI. 


\section{QUESTIONS}

1. Which square has the greater area-one with sides half an inch long or one with an area of half a square inch ?

2. A bagatelle board is made up of a reciangular portion and a semi-circular end. The extreme length of the board is $8 \mathrm{ft}$. and the width is $1 \mathrm{ft} .8 \mathrm{in}$. How many square feet of cloth would be required to cover the board?

3. Describe two methods of finding the area of a regular fivepointed star.

4. A " piece" of wall-paper is $12 \mathrm{yd}$. long and 21 in. wide. How many pieces would be required to cover the walls of a room $16 \mathrm{ft}$. long, $12 \mathrm{ft}$. wide and $9 \mathrm{ft}$. 6 in. high ?

5. What would be the cost of covering the floors of the following parts of a house with cork linoleum at $3 s .3 d$. a square yard allowing $10 \%$ loss for fitting?

$\begin{array}{ll}\text { Hall } & 12 \mathrm{ft} \text {. by } 5 \mathrm{ft} .6 \mathrm{in} . \\ \text { Passage } & 15 \mathrm{ft} \text {. by } 2 \mathrm{ft} .9 \mathrm{in} \text {. } \\ \text { Kitchen } & 13 \mathrm{ft} \text { by } 11 \mathrm{ft} .8 \mathrm{in} .\end{array}$

6. A carpet $16 \mathrm{ft} .6$ in. long and $13 \mathrm{ft} .6 \mathrm{in}$. wide costs $\mathfrak{L} 6.17 s .6 d$. What was the price per sq. yd. ?

7. An ordinary brick measures $9^{\prime \prime}$ by $4 \frac{1}{2}$ " by $2 \frac{1}{4}$. . How many bricks would be required to build a wall $30 \mathrm{yd}$. long, $10 \mathrm{ft}$. high and $18 \mathrm{in}$. thick ?

8. Find the number of litres of air in a room 12.8 metres in length, 9.3 metres in width and 3.5 metres high.

9. A cubic foot of water weighs $62.4 \mathrm{lb}$. What is the total weight of water in a reservoir, expressed in tons, the dimensions of the reservoir being: Length, 35 yd.; Breadth, 22 yd. 2 ft.; Depth, $10 \mathrm{ft}$. ?

10. The diameter of a cylindrical tank is $5 \mathrm{ft} .6 \mathrm{in}$. and its depth is $4 \mathrm{ft} .9 \mathrm{in}$. If a cubic foot of water weighs 1000 ounces, find the weight of water the tank will hold, expressed in tons. 


\section{CHAPTER III}

\section{MEASUREMENT OF WEIGHT}

24. Before considering the subject proper of this chapter, we will deal with the meanings of some words in common use, with especial reference to their application in scientific work. First on our list we will put the word "matter". Much learning has been expended on the exact definition of matter, but for our purposes it will be sufficient to consider that "Matter includes everything that we can handle". Thus iron, water, air are all forms of matter, since we can handle them, weigh them, and preserve them for further examination. On the other hand thoughts, heat, and sleep are not composed of matter, since we cannot so treat them.

The word "substance" means "kind of matter". Thus iron, water, and air are substances and when we say that iron is a hard substance we mean that all pieces of iron are hard, not simply that one special sample exhibits this quality. When we wish to speak of a special piece of any substance we call it a "body". Thus we say that a $56 \mathrm{lb}$. iron weight is a hard body, made of the substance iron.

Each body that exists contains a certain quantity of matter. For example, the $56 \mathrm{lb}$. weight mentioned above contains a certain quantity of iron, and, as long as the weight is protected from the action of substances or forces which may damage the iron in any way, the quantity of iron present in the weight does not alter, as far as we are able to judge in the present 
state of our knowledge. The "quantity of matter" contained in any given body is termed its "mass" and the unalterability of this mass is usually expressed by the phrase "the mass of a body is constant".

25. We all know that a body, left unsupported in the air, moves rapidly towards the Earth's surface and finally comes to rest on that surface. It is probably not so generally known that, while the body is travelling towards the Earth, the Earth is at the same time moving towards the body. This is actually the case, the rates at which the Earth and the body move being inversely proportional to their masses, whence it results that, the Earth's mass being so much greater than that of the body, the former's rate of movement is so insignificant that we cannot realise it.

Similarly any two bodies would move toward one another if causes preventing this motion did not intervene. We call "that which produces (or tends to produce) motion in a body", a force, and the mutual tendency of all bodies to move towards one another is said to be produced by the force termed "gravitation" or, more briefly, by the "force of gravity". To the famous philosopher, Sir Isaac Newton, we owe the discovery that the force of gravity is universal, and it was the application of his discovery to astronomical problems that first gave scientists a clear insight into the means by which the heavenly bodies are made to preserve their relative positions with respect to one another.

The universality of gravity must be clearly grasped. If two objects-say two footballs-having no initial motion were subject to no force save their own mutual gravitational attraction, they would move towards one another till they touched, and would remain in contact. 


\section{Measurement of Weight}

That they do not do so when placed near each other is a matter of everyday experience. The mutual pull between the Earth and each ball is so much greater than the pull of the balls on one another that their movement towards each other is entirely checked, and thus the existence of the gravitational force between them is completely masked. Experiments have been devised, however, which clearly show that force is exerted between bodies independently of the force exerted upon them by the Earth, but the consideration of such experiments is outside the scope of this book and the student must take the universal distribution of the force of gravity as proved, without requiring actual demonstration.

26. Since the pull of the Earth upon a body is so much greater and more important than any other force due to gravity, it is quite usual to speak of the "force of gravity" as if it applied solely to the mutual force exerted between the Earth and each object on or near its surface. We may measure the magnitude of the force of gravity in the case of any particular body by balancing it against another force. The spring-balance is a familiar instrument designed to this end. It consists essentially of a tightly-coiled metallic spring to which is attached at its upper end a ring by which it may be held in a vertical position, while at the lower end is a hook or a scale-pan by means of which the force of gravity exerted on a body attached thereto may be caused to stretch the spring. The stretching is resisted by the force produced by the tendency of the coil to resume its original shape, and, for amounts of stretching which are too small to distort the spring permanently, the increase of length is proportional to the stretching force. Thus, if one 
cubic inch of lead be placed in the pan, the spring will lengthen to a certain extent, and this length may be noted by marking the distance a metal indicator connected with the lower end of the spring moves along a vertical scale attached to the ring. If a second cube of lead, similar in all respects to the first, be now placed in the pan, the further increase in length of the spring will be found to be equivalent to that first noted. That is to say, the pull of the Earth on two cubic inches of lead is twice as great as its pull on one cubic inch. Three cubes will cause three times the elongation produced by one cube, four cubes four times, and so on. Each cube contains the same quantity of matter, i.e. has the same mass, and hence we see that the force exerted between the Earth and any given body is proportional to the mass of the latter-a body of twice the mass of another being pulled twice as hard towards the Earth's surface, one of ten times the mass ten times as strongly, and so on.

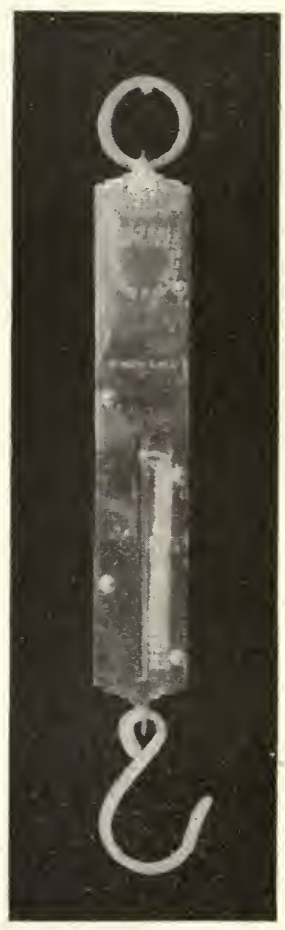

Fig. 18.

The pull exerted by the Earth upon a body in virtue of its mass is called its "weight", and the above experiment shows that, at any given point on the Earth's surface, the weight of a body is proportional to its mass, and vice versa. 
27. It is interesting to note that, although the mass of a body is constant, and its weight at any place is proportional to its mass, the weight of a body is not constant, but undergoes slight variations when its situation on the Earth's surface is changed. At the Poles a body would weigh about one-half per cent. more than at the Equator, e.g. a body weighing $16 \mathrm{lb}$. as shown by a spring-balance at either Pole, would only indicate about $15 \mathrm{lb} .15 \mathrm{oz}$. when similarly tested at the Equator. In explanation of this we may briefly say that it is due to the combined effect of two causes : (a) The Polar radius of the Earth is less than the Equatorial. Thus the distances between the centres of gravity of the body and the Earth vary, and the force exerted between two bodies is increased when the distance between their centres of gravity is lessened (see Ch. vI). (b) At the Equator the Earth's surface is rotating at over 1000 miles an hour and bodies resting on, or situated near, its surface tend to leave that surface on account of what is usually termed "centrifugal force", thus appearing slightly lighter than in higher latitudes where the rate of rotation, and hence the centrifugal force, is less.

28. Let us now suppose that, instead of placing a cubic inch of lead on the pan of our spring-balance, we gradually pour tea into the pan. The spring will stretch as before, and, when a certain quantity of tea has been poured in, the elongation produced will be exactly the same as that produced by the leaden cube. From what has already been said, it is clear that the weight of the tea now in the pan is equal to that of one cubic inch of lead. Applying the principle that, at any given place, the weight of a body is proportional to its mass, we arrive at the conclusion that the masses 
of the tea and the lead are also equal. The volumes occupied by the two bodies are very different but the "quantity of matter" in each is the same. We are thus led to the idea that, if we have a method of comparing the weights of any two bodies, we are at the same time able to compare their respective masses. This point is of importance since, when we purchase tea, sugar, butter and other commodities by weight, as we usually term it, we are not primarily concerned with the pull exerted between the Earth and the article purchased, but with the quantity of the article which we obtain. The instrumentin general use for the comparison of weights - and consequently of masses - is the balance. Many forms of this exist but the principle upon which the working of each depends is the same in all cases.

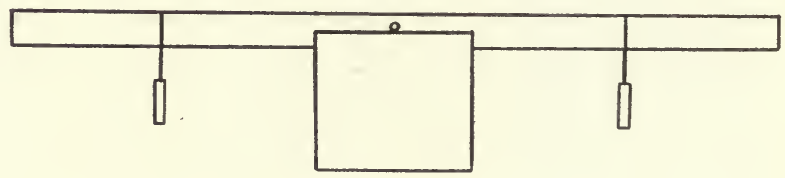

Fig. 19.

Experiment 13. Bore a hole through a long wooden rod-a boxwood metre scale will do excellently - carefully at right angles to its length and midway between the two ends. Its diameter should be such that a knitting-needle, when pushed through, fits closely. Support the ends of the needle upon two thick wooden blocks-or large books-and note whether the rod remains in a horizontal position. If it does not, fold a piece of lead foil-the sort in which chocolates are wrapped will serve-around the end which is higher, and, by sliding the foil along the rod, find a position 
for it such that, when the rod is again left free to move, it takes up a horizontal position ${ }^{1}$.

Tie a fine thread of cotton around a new penny and make a loop in the free end of the thread of sufficient size to slide freely along the rod. Hang the penny from the rod at any point on one arm. On the other arm hang another new penny, similarly tied with an equal length of the same sort of thread. Arrange the position of the second penny so that the rod is again horizontal. Measure carefully the distance from the centre of the top edge of the rod above the needle to the point from which each penny is suspended. Note and compare the measurements obtained. (If a metre scale be used the lengths required may be readily read off directly from the graduations of the scale.)

Move the first penny to a new position on the rod and adjust the position of the second till the rod is once more horizontal. Note and compare the distances from the centre of the rod to the point of suspension of each penny.

Next hang two pennies, using a double allowance of thread, on the rod in place of the second penny, leaving the first on the other arm. Adjust till horizontality is attained and note and compare the distances measured as before. Repeat the above operations, using varying numbers of new pennies on each arm of the rod.

On carefully comparing the results obtained, it will be at once seen that the further a penny is hung from the point of support at the centre of the rod (usually termed the "fulcrum ") the greater is its

1 Greater delicacy of adjustment may be attained if two thin glass plates be interposed between the ends of the needle and the upper faces of the blocks. 
power of turning the rod about the fulcrum. For instance, if one penny be placed on one arm $20 \mathrm{~cm}$. from the centre, it will be found to balance two placed $10 \mathrm{~cm}$. from the centre. That is to say, the turning power of each of these latter pennies is only one-half that of the single penny situated twice as far from the fulcrum. Again, if we put penny No. $140 \mathrm{~cm}$. from the middle of the rod, it will be found that we require four pennies on the other arm at $10 \mathrm{~cm}$. from the centre to balance it, i.e. by doubling its distance from the point about which the rod turns we have doubled its turning-power.

The turning-power of a force exerted in any similar manner to that illustrated in the above experiment is termed the " moment" of the force about the turningpoint, and the conclusion which may be drawn from such results as the above is generally summarised as follows :

When the moments of two forces tending to turn a body in opposite directions about a given fulcrum are equal, the body remains at rest.

This is the simplest case of a general principle of much wider application, known as the "Principle of Moments", and may be put in a brief mathematical form thus :

1st force $\times$ its distance from fulcrum

$$
=2 \text { nd force } \times \text { its distance from fulcrum. }
$$

29. We are now ready to understand why an ordinary chemical balance, or the less delicate instrument known as a "pair of scales", may be used to determine the weight and hence the mass of a body. Essentially a balance consists of a "beam", able to swing freely about its middle point, with pans attached 
to its ends in which the bodies to be weighed may be placed. In a simple chemical balance, as shown in Fig. 20, the beam is made of brass and turns upon one edge of a polished triangular prism of agate-a hard stone-which is supported upon a flat surface of the same material when the balance is in use. Near the ends of the beam are two similar "knife-edges" of agate, with their edges directed upwards, parallel to, and equidistant from, the middle knife-edge. Upon these are hung the pans of the balance by means of

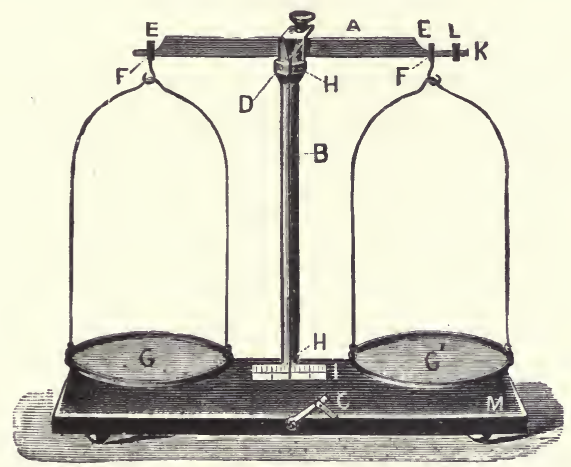

Fig. 20.

stirrups, the upper inside surfaces of which are also made of agate. Depending from the middle of the beam in front of the stout central support is a long pointer, the lower end of which moves over a graduated scale attached to the wooden base of the balance. In front of the base is a milled brass head or a small brass handle which may be turned from left to right and vice versa. This works the "arrestment" of the balance, a mechanical arrangement whereby the knifeedges on the beam are kept out of contact with the centre agate plane and the inner portions of the stirrups, 
when the balance is not in use. From one end of the beam-in some forms of balance from each end-projects a thin brass rod with a fine screw-thread upon its surface. A milled nut travels along this rod. Such a balance as has been described is usually kept within a wooden case with glass sides, in order to protect its parts from the action of damp air and to keep them as free as possible from dust.

The balance is used to determine equality of masses. Any body placed in one pan is attracted downwards by gravity with a force proportional to its mass. If another body or bodies be placed in the other pan and it be found that the beam of the balance comes to rest in a horizontal position, we know, since the arms of the beam of the balance are of equal length, that the force with which the contents of the second pan is attracted downwards is equal to that acting on the first body, in accordance with the Principle of Moments. Since these forces-the weights of the respective bodiesare equal, we infer that the masses of the bodies in the pans are also equal.

30. In order to simplify measurements of weight, units of weight have been adopted, just as is the case of the other classes of measurements previously dealt with. The British standard unit, the "pound", may be defined as "the weight of a piece of platinum, weighed in a vacuum at the temperature of melting ice, which is kept in the Standards Department of the Board of Trade". The need for specifying the exact conditions under which the weighing must take place will be grasped when the chapters dealing with displacement of fluids and the effect of heat on bodies have been read. Multiples and submultiples of the standard unit are given in the subjoined table. 


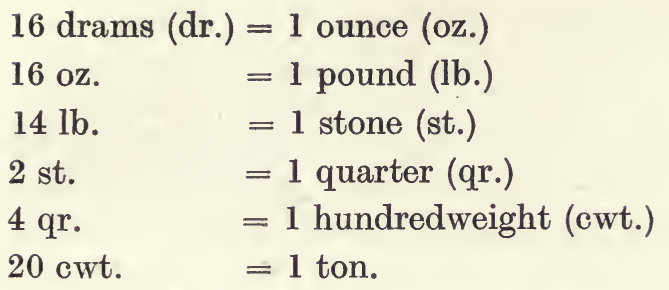

The standard unit of weight in the Metric System is that of the "kilogram", a lump of platinum kept at the Archives in Paris. This was constructed so as to have the same weight as a cubic decimetre of pure water when weighed at its temperature of maximum density-about $3.9^{\circ} \mathrm{C}$. The thousandth part of a kilogram is termed the "gram" and the Metric table of weights is as follows :

$\begin{array}{ll}10 \text { milligrams (mg.) } & =1 \text { centigram } \\ 10 \text { centigrams } & =1 \text { decigram } \\ 10 \text { decigrams } & =1 \text { gram }(\mathrm{g} .) \\ 10 \text { grams } & =1 \text { decagram } \\ 10 \text { decagrams } & =1 \text { hectogram } \\ 10 \text { hectograms } & =1 \text { kilogram }(\mathrm{Kg} .) \\ 10 \text { kilograms } & =1 \text { myriagram. }\end{array}$

One kilogram $=2 \cdot 2 \mathrm{lb}$. (approximately).

It will be again observed that the Metric table excels in simplicity as compared with our own, and further, that a fairly simple relation has been established between the units of length and of weight by the adoption of a standard substance-pure waterin determining the standard of weight. No such simple relation was established in the British System, although it is useful to remember that a cubic foot of water weighs almost exactly 1000 ounces. 
Since weight is directly proportional to mass, it is unnecessary to adopt a special system of units of mass, and mass is consequently generally expressed in the same units as weight, viz. pounds and kilograms. To avoid confusion, however, it must be clearly understood that the term pound, when used as a unit of weight, denotes a force, while when used as a mass unit, it indicates only a definite quantity of matter.

31. Practice in the use of the balance will be provided in the experiments which follow.

Experiment 14. Examine carefully the box of weights supplied with a balance such as that shown in Fig. 20. This usually contains the following weights :

50 g., 20 g., 20 g., 10 g. ; 5 g., 2 g., 2 g., 1 g.

0.5 g., $0 \cdot 2$ g., 0.2 g., $0 \cdot 1$ g. ; $0 \cdot 05$ g., 0.02 g., $0 \cdot 02$ g., $0 \cdot 01$ g. With a balance and box of weights of this character, bodies weighing up to 100 grams may be weighed, and the error involved in weighing should not usually be greater than that of the smallest weight, $0.01 \mathrm{~g}$. or $10 \mathrm{mg}$., if the balance is in good order.

Turn the arrestment so that the pans of the balance are set swinging. This is called "putting the balance into action". Note the number of divisions over which the end of the pointer swings on each side of the centre division of the scale. The lengths of swing gradually lessen till the beam comes to rest. To avoid the waste of time involved in waiting till this occurs it is sufficient to note whether the number of scale-divisions passed over on each side of the centre is approximately the same. Thus, if the reading on the right of the middle of the scale be 7 divisions, that on the left 6.5 , followed by another on the right of 6 divisions, the balance is in good working order and needs no special adjustment 
before use. Should the number on one side, however, exceed that on the other by more than 2 or 3 divisions, the milled nut at the end of the beam is turned on its rod till the pointer swings evenly on each side of the centre of the scale.

\section{Exercise for Student.}

In which direction must the nut at the right-hand end of the beam travel if the pointer swings too far to the right, showing that the left side of the balance is slightly the heavier? Apply the Principle of Moments.

While adjusting the balance, and while adding bodies to, or removing them from, the pans, always put the balance out of action. This precaution prevents undue wear and tear of the knife-edges, upon the proper condition of which the accuracy of the weighings obtained largely depends.

When the balance is in proper adjustment, place a penny on the left-hand pan. Open the box of weights and remove from it, with the forceps supplied with the box, a weight estimated to be greater than that of the penny. Place this on the right-hand pan and gently raise the arrestment with the left hand. Note the direction of swing of the pointer. If the weight prove too heavy, put the balance out of action, remove the weight, and replace it by the next smaller weight. Again raise the arrestment and note the indication of the pointer. Continue adding weights to, or removing them from, the right-hand pan, as directed by the pointer indications, dealing with them in the descending order of magnitude in which they are arranged in the box, till the smallest weight is reached, and the pointer swings nearly evenly on each side of the middle point of the scale. If the balance is cased, the front should now be lowered, the arrestment again 
raised, and a reading of the pointer indications made while the balance is thus protected from the effects of accidental currents of air. Should this second reading show that the weight has been obtained as nearly as can be effected with the balance and weights used, add up the weights on the pan, note the total and then replace them in order in their proper compartments in the box, checking the total weight found while so doing. Close the front of the balance and the lid of the box when the weighing is finished.

Experiment 15. Place a $1 \mathrm{oz}$. weight on the lefthand pan of the balance and find its weight in grams by the method of the previous experiment. From the result find by calculation the weight of 1 kilogram in pounds and compare your value with that shown under the table of Metric weights previously given.

The balance may be used to determine indirectly the areas of irregular or curvilinear figures such as those of which the areas have been found by other methods in previous experiments, or to check the results obtained for regular figures by calculation from their dimensions.

EXPERIMENT 16. Obtain some tough millboard of medium thickness and of good quality, or some thin sheet zinc. Cut carefully from one of these media a square of definite area, say $4 \mathrm{sq}$. in. or $25 \mathrm{sq}$. cm. Weigh this as accurately as possible and from the result determine the weight of $1 \mathrm{sq}$. inch or $1 \mathrm{sq}$. $\mathrm{cm}$. of the material used. Trace the outline of an irregular figure, of which the area is to be found, on the millboard or zinc. Cut out the figure as neatly as you can and weigh it. Assuming that the weights of the figures are proportional to their areas, the area of the figure 
in sq. in. or sq. cm. may be obtained with a fair degree of exactness by dividing the weight of the figure by the weight of unit area as previously determined. Compare this with the area as found for the same figure by the method of counting the squares described in Experiment 7.

\section{Exercise for Student.}

What must be assumed with respect to the nature of the millboard or zinc used for carrying out this experiment?

32. The accuracy of graduation of the pipettes, burettes, or other measuring-vessels used for the determination of liquid volumes may be tested by means of a good balance.

Experiment 17. Place a light glass vessel, such as a beaker, on the left-hand pan of the balance. On the other pan place a similar vessel. If they do not exactly balance one another, gradually add small leaden shot, or clean dry sand, to the pan on which the lighter beaker stands until an exact balance is obtained. This operation is known as "counterpoising" and is generally resorted to when the actual weight of a containing vessel or other body is not required to be definitely known for the purposes of an experiment. The second beaker may be dispensed with, if desired, and dry sand or shot alone used in effecting the counterpoise. Remove the first beaker from the pan to the working-bench. Insert the jet of a 10 c.c. pipette into a quantity of distilled water in another vessel, place the mouth at the upper end of the pipette, and suck gently till the water has risen above the graduation mark on the stem of the instrument. Remove the end from the mouth and quickly place the 
forefinger over the open end (see Fig. 21). Leaving the jet of the pipette just below the surface of the water, slightly release the pressure of the finger, thus allowing the water-level to sink slowly to the graduation mark. When the lowest point of the meniscus touches themark, press the finger firmly on the opening at the upper end of the pipette, raise the latter from the water, and insert its lower end in the counterpoised beaker. Remove the finger and the water will run into the beaker. Do not blow out the last drop but allow the pipette to drain in a vertical position with its jet

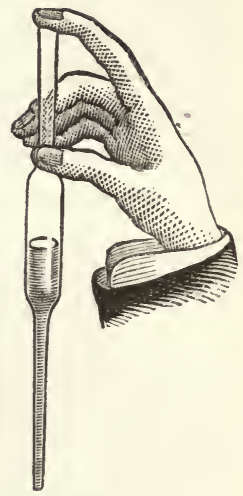

Fig. 21. against the wet side of the beaker for 15 seconds. Place the pipette on the bench and transfer the beaker and its contents to the left-hand scale-pan. Put a $10 \mathrm{~g}$. weight on the right-hand pan and raise the arrestment. This weight should be found to bring about an almost exact balance. Should it not do so, find the weight required to effect a balance. If it be found to vary from $10 \mathrm{~g}$. by more than $03 \mathrm{~g}$., the pipette is probably improperly graduated. Measuring-vessels for liquids are usually graduated to deliver the volume marked on them when the contained liquid is at a temperature of either $15^{\circ} \mathrm{C}$. or $60^{\circ} \mathrm{F} .10$ c.c. of water at this temperature weigh slightly less than $10 \mathrm{~g}$.- - to be exact $9.99125 \mathrm{~g}$. The type of balance supposed to be used will not show. such small differences of weight as ${ }^{\cdot} 00125 \mathrm{~g}$. so that, for our purposes, the weight of the water should be $9.99 \mathrm{~g}$. Since, however, we are not using a very expensive balance nor very accurately adjusted weights, a pipette which delivers a quantity of water at $15^{\circ} \mathrm{C}$., 
the weight of which lies between 9.97 and $10.01 \mathrm{~g}$., would not be considered to require re-graduation.

Similarly test the accuracy of graduation in the case of 5 c.c., 20 c.c., 25 c.c., and 50 c.c. pipettes.

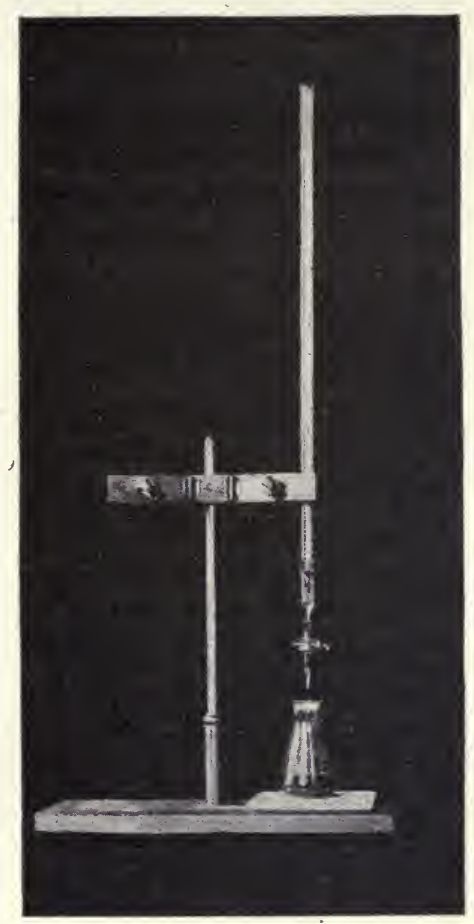

Fig. 22.

Experiment 18. To check the accuracy of graduation of a burette, counterpoise a beaker sufficiently large to hold all the liquid in the burette. Fill the burette with distilled water till the water-level is above the zero mark, open the tap and allow water to run out till the lower edge of the meniscus just reaches the 
topmost graduation. Clamp the burette in an upright position in a suitable stand (Fig. 22). Place the beaker under the tap and allow exactly 5 c.c. of water to run from the burette into the beaker. Close the tap and weigh the contents of the beaker as in the last experiment. Add water from the burette, 5 c.c. at a time, weighing the contents of the beaker after each addition, till the lowest graduation mark on the burette is reached. A properly graduated instrument should show equal increments of weight for each successive.quantity run out, and the weight of each such quantity should be very approximately $\mathbf{5}$ grams.

\section{Exercises for Student.}

1. How would you propose to test the accuracy of graduation of a flask constructed to hold 50 c.c. of liquid ? Carry out the test by the method you suggest.

2. How would you graduate a flask so that it shall contain 80 c.c. of water at ordinary temperatures? Obtain a flask of suitable size and carry out the graduation by your suggested method.

3. How would you check the accuracy of graduation of such a flask as that mentioned in the previous exercise?

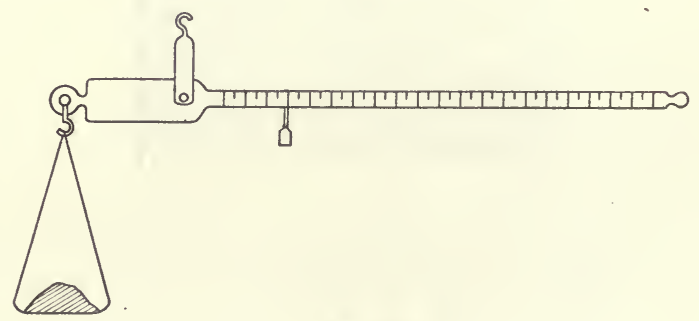

Fig. 23.

33. A weighing-machine, such as one of those used for finding the weight of a person commonly seen in 
chemists' shops, affords another example of the application of the Principle of Moments. It is constructed on the model of the simple steelyard, of which an illustration is shown in Fig. 23. It resembles an ordinary balance in that it has a beam pivoted about one point but this point is not situated at the centre of the beam, being much nearer to one end than the other. Let us suppose that the point about which the beam turns is $2^{\prime \prime}$ from one end of the beam and $20^{\prime \prime}$ from the other. Applying the Principle of Moments, it is evident that a weight of $1 \mathrm{lb}$. attached to the end of the longer arm will balance a weight of $10 \mathrm{lb}$. at the other end of the beam. When placed halfway along the longer arm, the $1 \mathrm{lb}$. weight will balance $5 \mathrm{lb}$. at the other end, and so on. The.longer arm of the beam is usually graduated in such a way that the weight of the object attached to the end of the shorter arm may be read directly from the scale when an exact balance has been effected, by noting the position of the weight which slides along the longer arm. In the weighing-machines used to determine the weights of heavy objects, a set of weights, suspended from the end of the long arm, is used in conjunction with the sliding weight. They are marked with values which bear the same ratio to their actual weights as the length of the longer arm bears to that of the shorter. Large machines of this character may be found in market-places, where they are of use in weighing cattle, loads of hay, and similar heavy bodies. 


\section{QUESTIONS}

1. One arm of a weighing-machine is $10 \mathrm{ft} .6 \mathrm{in}$. long and the other $1 \mathrm{ft}$. What would be the actual weights of the lumps of iron used with this machine marked " 10 cwt.", " $56 \mathrm{lb."} \mathrm{?}$

2. In an ordinary steelyard a weight of $1 \mathrm{oz}$. balances a weight of $1 \mathrm{lb}$. suspended from the end of the shorter arm. The length of the long arm is $12^{\prime \prime}$. Find the length of the short arm.

3. A $56 \mathrm{lb}$. weight is suspended from a stout wooden beam at a distance of $2 \mathrm{ft} .6 \mathrm{in}$. from the centre of the beam, which is supported at its middle point so that it is free to turn about that point. Where must a weight of $35 \mathrm{lb}$. be hung on the other half of the beam in order to maintain the beam in a horizontal position ?

4. Find by calculation the number of ounces in 6 kilograms and the number of grams in a hundredweight.

5. An ordinary brick measures $9^{\prime \prime} \times 4 \frac{1}{2}{ }^{\prime \prime} \times 2 \frac{1}{4}$ and is twice as heavy as the same volume of water. If a cubic foot of water weighs $62.4 \mathrm{lb}$., what is the weight of a brick?

6. A sheet of cardboard $7^{\prime \prime}$ long and $5^{\prime \prime}$ wide weighs $22 \cdot 78 \mathrm{~g}$. A circle cut from the same sample of cardboard weighs $34.60 \mathrm{~g}$. Find the radius of the circle.

7. 50.0 c.c. of clay weigh $95.0 \mathrm{~g}$. What weight of clay is contained in a cylinder of that material $50.0 \mathrm{~cm}$. long with a diameter of $5 \cdot 0 \mathrm{~cm}$. ?

8. Give a detailed description of the method you would adopt in order to find as exactly as possible the weight of 1 inch of a sample of fine copper wire by means of a balance which is only capable of weighing bodies under $25 \mathrm{~g}$. in weight to the nearest centigram.

9. A see-saw is formed by balancing a plank $12 \mathrm{ft}$. long about its centre on a wooden trestle. A child weighing $60 \mathrm{lb}$. sits on one end of the plank, and is balanced by a man weighing 10 st. Where must the man be seated? 


\section{CHAPTER IV}

\section{THE RELATION BETWEEN WEIGHT AND VOLUME}

34. It is a matter of common knowledge that a pound of lead occupies less space than a pound of sugar and that an ordinary brick weighs much less than a lump of iron of the same size. We usually express our realisation of these facts by such phrases as "Lead is heavier than sugar " or " Bricks are lighter than iron". In this chapter we deal with this matter from the standpoint of science by making exact measurements of the relation which exists between the volume of a body and its weight. We shall learn that the above expressions are somewhat loose in wording and should more strictly be stated thus: "Lead is denser than sugar"; "Bricks are less dense than iron".

Experiment 19. Carefully determine the volumes in c.c. of three solid figures made of different materialssay lead, iron, and wood-by one of the methods previously given. Weigh each body as described in the last chapter. Find the weight in grams of 1 c.c. of each substance by dividing the weight of each body by the corresponding volume. Compare the results obtained in this manner for the weights of equal volumes of the three substances.

Repeat the experiment with three different solid figures, each made from one of the three materials used previously. Find the weight of 1 c.c. of each substance as before and compare the results obtained with those previously determined.

If the experiment has been carried out with sufficient care, it will be noted that the weight-and hence also 
the mass-of 1 c.c. of each material is practically the same for both examples and is evidently independent of the size and shape of the particular body dealt with. A corresponding statement to this will still hold good if our measurements of volume and weight are expressed in different units, e.g. cubic inches and ounces, or cubic feet and kilograms.

Thus, if our three figures were made of wood, iron, and lead as suggested, our results will be something like this :

1 c.c. of lead has a mass of $11 \cdot 2 \mathrm{~g}$.

$\begin{array}{llll} & \text { iron } & & 7 \cdot 8 \mathrm{~g} . \\ & \text { wood } & & 0 \cdot 70 \mathrm{~g} .\end{array}$

If our measurements be made in cubic inches and ounces, we shall have :

$1 \mathrm{cu}$. in. of lead has a mass of $6.47 \mathrm{oz}$.

$\begin{array}{llll}, & \text { iron } & & 4.51 \mathrm{oz} . \\ , & \text { wood } & , & 0.404 \mathrm{oz} .\end{array}$

In cubic feet and kilograms we should obtain: $1 \mathrm{cu}$. ft. of lead has a mass of $317 \mathrm{Kg}$.

$\begin{array}{llll}" \text { iron } & & 221 \mathrm{Kg} . \\ " & \text { wood } & , & 19 \cdot 8 \mathrm{Kg} \text {. }\end{array}$

To denote the mass of unit volume of a substance the name "density" is used. Thus the density of lead may be variously stated as $11 \cdot 2$ g. per c.c., $6 \cdot 47 \mathrm{oz}$. per cu.in., or $317 \mathrm{Kg}$. per cu. ft. In each case the units of mass and of volume must be clearly stated and it will be noted that the numerical portion of the statement varies with each choice of units made.

Exercise for Student.

Determine by calculation

(a) The density of lead in grams per cu. dm.

(b) " $\quad$ iron in kilograms per c.c.

(c) " wood in lb. per cu. yd. 
35. In practice it is usual to compare the densities of all substances with that of a standard substance, the standard in general use being the density of pure water. In the last chapter we learnt that the weight of 1 c.c. of pure water was 1 gram; hence the density of water is $1 \mathrm{~g}$. per c.c. The densities of lead, iron, and wood, as given above, are respectively $11 \cdot 2,7 \cdot 8$, and 0.7 times the density of water. To the numbers $11 \cdot 2,7 \cdot 8$, and $0 \cdot 7$, when used in this connection, a special term is applied. They are called the "specific gravities" of lead, iron, and wood. This name is not happily chosen. It may be paraphrased as "special. weights". A specific gravity is not, however, a weight but simply a number, and a much more apt designation for it is "relative density". Thus we may define the specific gravity or relative density of a substance as "the number of times that the density of the substance is as great as the density of pure water", or as "the ratio between the density of the substance and that of pure water".

36. The specific gravity of a substance is sometimes confused with its density. This confusion should not arise, however, if it be remembered that the specific gravity is merely a number, being the value of a ratio, e.g. the specific gravity of iron is $7 \cdot 8$; while the density is a measure of the mass of unit volume of the substance and requires for its complete statement that mention be made of the names of both the unit of mass and of volume involved, e.g. the density of iron is $7 \cdot 8 \mathrm{~g}$. per c.c., or $4.51 \mathrm{oz}$. per cu. in.

\section{Exercise for Student.}

Why is the number of grams per c.c. in the statement of the density of a substance the same as the number which denotes its specific gravity?

H. D. S. 
37. A further method of determining the density or the specific gravity of solid substances will be found fully described in Chapter vi. We will next consider a simple method of determining the density of a liquid substance.

Experiment 20. Counterpoise carefully (p. 577) a small long-necked flask with a narrow strip of paper gummed along its neck, a horizontal line being drawn across the paper at any point. Pour some of the liquid of which the density is required into the flask till the lower edge of the meniscus just touches the mark on the paper strip. Weigh the liquid by adding weights to the other pan. Pour out the liquid and wash out the flask with several successive quantities of tap-water to ensure that no appreciable amount of the liquid originally present remains in the flask. Rinse the flask out twice with small quantities of distilled water, pour in distilled water till the mark on the neck is reached as before, and wipe the outside of the flask quite dry. Weigh the distilled water.

Since $1 \mathrm{~g}$. of distilled water at ordinary temperatures occupies almost exactly 1 c.c., the number of grams of water contained in the flask is the same as the number of c.c. in the interior volume of the flask up to the mark. The liquid and the water both occupied this volume, hence the volume of the liquid is known. The mass of the liquid having been already determined, its density is a matter of simple calculation.

\section{Exercise for Student.}

What is the specific gravity of the liquid of which you have just determined the density ?

38. To save time in the determination of liquid densities, small flasks are constructed to hold, at ordinary temperatures, a known weight-say 10, 25, 
or $50 \mathrm{~g}$. of water. In using these, the weighing of the distilled water may be dispensed with, provided that the flasks are carefully made. These vessels are called "density flasks" or "pyknometers" (Gr. pyknos, dense) and two common forms are shown in Fig. 24.

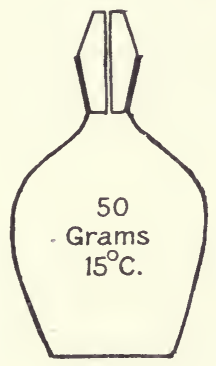

A

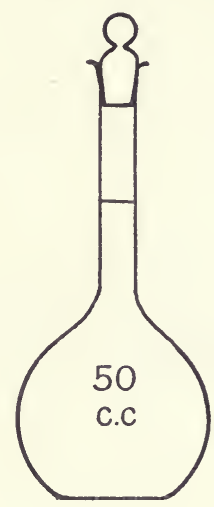

B

Fig. 24.

Of these $A$ represents the better form but its use demands more careful manipulation. If such a flask is available, the student should perform

Expertment 21. Weigh the flask empty, after first making sure that it is quite clean and dry, both within and without. . Completely fill it with the liquid to be dealt with. Holding the flask with a clean duster, push the perforated stopper home. Remove the excess of liquid from the outside of the flask with the duster, care being taken to leave the hole in the stopper quite full of liquid and to avoid touching the flask directly with the warm hand. Weigh the flask and its contents. Subtracting the weight of the empty flask from the weight of flask and liquid gives the weight of liquid 
required to exactly fill the flask. From the weight of the water marked on the exterior of the flask the interior volume may be found and the.density of the liquid may thus be readily ascertained. As a check on the accuracy of the value marked on the flask, it may be rinsed out thoroughly with tap-water followed by distilled water and then filled with distilled water and weighed. If the water is at or very near the temperature at which the flask is supposed to contain the weight marked on it, the number of grams of water it holds should be the same as the number of grams marked on the flask.

Other methods of determining the density of a liquid will be described in later chapters.

39. The density of a gas, such as air or carbon dioxide, cannot be accurately determined by any simple means. A method which gives roughly approximate results for air will be found fully described in Chapter $\mathrm{x}$. Experiment and further consideration of this point may be postponed till that stage of the work is reached. The density of air being known, the density of other gases may be found by such methods as that described in Chapter $\mathrm{x}$.

40. The approximate specific gravities of some common substances are given in the table below:

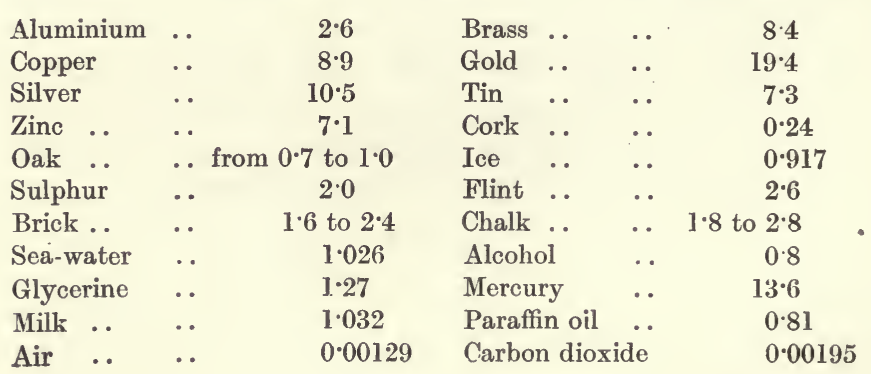




\section{QUESTIONS}

1. Which has the greater weight - a cube of silver of which the edge is $3 \mathrm{~cm}$. long or one of aluminium with an edge $5 \mathrm{~cm}$. in length ?

2. A cubic foot of water weighs $1000 \mathrm{oz}$. What is the weight of $100 \mathrm{cu}$. in. of ice?

3. Find the volume of a kilogram of mercury. What volume of glycerine would have the same weight as the mercury?

4. A density flask filled with water weighs $71 \cdot 67 \mathrm{~g}$. Filled with linseed oil, the combined weight of the flask and oil is $68.65 \mathrm{~g}$. If the weight of the empty flask is $21.35 \mathrm{~g}$., find the specific gravity of the oil.

5. What weight of mercury would be required to fill a glass tube 1 metre long with a bore of $1 \mathrm{~cm}$. ?

6. A rectangular box with square ends has sides twice as long as the ends. It holds $3200 \mathrm{~g}$. of alcohol. Find its length, breadth, and depth in centimetres.

7. A cubic centimetre of hammered platinum weighs $21 \cdot 25 \mathrm{~g}$. What volume of air has the same weight?

8. How many gallons of water could be contained in a tank $12 \mathrm{ft}$. long, $5 \mathrm{ft}$. wide and $6 \mathrm{ft}$. deep ?

9. 7500 c.c. of water are frozen solid. What volume of ice is produced? What is the weight of the ice?

10. Correct, where necessary, the following statements :

(a) Ice is lighter than water.

(b) 1.037 is a high value for the density of milk.

(c) The specific gravity of chalk is $2 \cdot 3$ grams per cubic centimetre, and is less than that of sulphur. 


\section{CHAPTER V}

\section{THE CONSTITUTION OF MATTER}

41. In the previous chapters the words "solid", "liquid", and "gas" have been used freely, and there has probably been no difficulty in understanding what was implied by these terms. It will be well, however, to take into consideration the distinguishing features of these three " states of matter "- as they are generally called-and to deal with one or two important properties peculiar to those states, before proceeding further.

Our common knowledge and some simple experiments will provide us with the necessary information on which to base clear ideas of what is meant by each of the above terms.

We all know that a solid body does not require the support of a containing vessel in order to maintain its shape, and that, unless the body is acted upon by some external force, it retains its size and shape indefinitely. It may be readily believed that, if a penny and a written description of its shape and size be sealed up in a glass vessel, an investigator examining it a thousand years hence would find that the description still held good.

Thus our first point with respect to the nature of solids is that they possess shapes and sizes which are only changed by the application of force.

EXPERIMENT 22. The spring of a spring-balance is another example of a solid body. It has a definite shape when the balance is not in use. Examine the spring of such a balance and describe its shape. Hang 
a suitable weight, say one-half the value of the greatest weight shown on the scale of the balance, from the lower end of the instrument and note the reading of the indicator on the scale. Describe the new shape taken up by the spring. Attach the upper end of the balance to a support and allow the weight to hang from it for a quarter of an hour. Take readings of the position of the indicator every three minutes. With a well-made spring, no measurable alteration of the reading will be noted. Measure the increase in length of the spring due to the attachment of the weight. Repeat the experiment, using another weight. Note the relation between the increase of length caused in this case and that caused by the former weight, and compare it with the ratio between the weights used.

In this experiment, the spring is changed in shape by the action of a force, the amount of change is definite for a given force and does not alter appreciably when the time during which the force acts is prolonged. Further, the amount of change produced is directly proportional to the force producing it.

In common with all other bodies, solids have definite masses, as already indicated in a previous chapter. The chief characteristics of a solid body may be summarised thus :

It has a definite mass; it retains its shape without lateral support; its volume and shape are in general not easily altered except by the action of a considerable force; when its shape is changed under the action of a force, the change produced is definite and dependent upon the force applied.

42. Let us next examine the qualities of a liquid.

ExPERIMENT 23. Half fill a small flask with water and cork it securely. Gum a strip of paper to the 
outside of the flask and mark on it the position of the upper surface of the liquid when the flask is standing in an upright position on the bench. Note the shape of the water occupying the lower part of the flask and the position of its free surface with respect to that of the Earth. Weigh the flask and its contents. Turn the flask so that it rests on its side instead of on its base. Note again the shape of the water and the position in which its free surface settles. Test whether any change in mass has accompanied the change in position of the flask. Replace the flask in the upright position and note whether the level of the watersurface has varied with reference to the mark on the gummed strip. Keep the flask and its contents securely corked for a week and again examine the position of the water-level and redetermine the mass of the flask and contained water.

As a result of the above investigation, we shall be led to the conclusions that a liquid has a definite mass ; that its volume does not vary when no external force is applied and the temperature is constant, but that its shape varies readily under the action of a force, i.e. a given mass of liquid has no definite shape of its own, but takes that of the portion of the vessel containing it. Further, its upper surface, if free (i.e. not in contact with the interior of the vessel), is always parallel to that of the Earth, or, in other words, lies in a horizontal plane ${ }^{1}$.

\section{Exercise for Student.}

What was the force which caused the water to change its shape when the flask was tilted?

1 I.e. except for the meniscus referred to above and the related convexity of surface when the containing vessel cannot be wetted. 
A further characteristic of liquid bodies which serves to distinguish them from solids is the fact that a given mass of liquid requires lateral support, i.e. it must be put into a vessel with sides sufficiently strong to support its weight. Unless thus supported, the liquid spreads itself under the influence of the force of gravity as a thin layer over the surface of whatever solid body is situated immediately beneath it. This point of distinction between solids and liquids is sometimes expressed by the statement that the smallest particles or molecules (see later portion of this chapter) of which a solid body is composed possess the property of cohering together firmly enough to afford each other mutual support, while, in the case of the molecules of a liquid, the cohesion between them is not sufficient to bring about this result.

43. A gas resembles a liquid in many of its properties, but has no free surface. Any quantity of a gas, however small, when introduced into a previously empty vessel, immediately extends in all directions to the furthest limits of the vessel, and a sample of the contents, taken from any region within the vessel, will be found to give the characteristic reactions of the gas introduced. Further, if the vessel be transparent, no free surface may be seen marking off the limit of the gas, as is the case with a liquid. A gaseous body, in common with all others, does, however, possess a definite mass. With regard to change of shape, it is a matter of common knowledge that a gas offers less resistance to deformation than a liquid, e.g. it is easier to move the hand backward and forward in air than in water.

The considerable similarity in behaviour of liquids and gases has led to their being classed together under the general name of "fluids". 


\section{Exercise for Student.}

From the information already supplied, make a summary of the general properties of fluids.

44. Solids may be further distinguished from fluids by the fact that the latter in most cases intermingle freely with one another by the process termed "diffusion", irrespectively of their densities. All gases exhibit this property, but some pairs of liquids, such as olive oil and water, do not, for other reasons, mix to any appreciable extent.

The following experiment should only be performed under the immediate supervision of a teacher.

Experiment 24. Dip a long glass tube of narrow bore into a bottle of bromine. (This is a liquid of a very dark red-brown colour, which gives off readily large quantities of dark orange-coloured vapour. This vapour is exceedingly harmful to the membranes of the throat and lungs and must on no account be breathed.) Close the top of the tube with the forefinger and remove the lower end of the tube from the liquid. Let the liquid run out of the tube by slightly releasing the pressure of the finger, till only enough is left to fill about an inch of the tube. Press the finger firmly on the upper end of the tube, cautiously remove the tube from the bottle, and lower it carefully to the bottom of a tall glass cylinder. Remove the finger when the lower end touches the bottom and let the liquid run out. Remove the tube and drop it gently into a large vessel of cold water. Put the stopper into the bottle of bromine. Cover the top of the cylinder with a greased glass plate and put it in a fume-chamber, or, if that be not available, on the sill outside a window. The liquid bromine soon becomes a gas, which is much 
denser than air and at first spreads over the bottom of the cylinder. Leave the apparatus untouched for some time, examining the appearance of its contents at intervals of 5 minutes. In spite of the considerable difference in the densities of the two gases, air and bromine, it will be found that the bromine vapour rises slowly in the cylinder, becoming less pronounced in colour as it does so on account of admixture with the air. If the duration of the experiment be sufficiently prolonged, the bromine, as shown by its colour, will eventually reach the top of the vessel, and the contents of the latter will be of a uniform pale brown tint.

Caution. When the experiment is finished, remove the glass plate from the top of the cylinder in the open air, and turn the vessel upside down at arm's length. The bromine vapour will fall from the cylinder. When washing the cylinder and glass tube in readiness for future use, be careful to keep the nose and eyes away from the sink till it is certain that no traces of bromine are left.

ExPERIMENT 25. Half fill a small glass dish with concentrated hydrochloric acid solution. Add sufficient litmus solution to a quantity of water to make the liquid of a deep blue tint. Fill a test-tube with this blue liquid, cover the mouth of the tube with the thumb, and invert the tube in the acid. Gently withdraw the thumb under the surface of the acid, and fix the tube in position by means of a clamp. The mouth of the tube should be about half an inch below the surface of the acid. The strong acid will not damage the skin if it be washed off at once. Allow the apparatus to stand in a quiet place for a day or two, examining the appearance of the blue liquid at 
intervals of about two hours during the daytime. It will be found that the lower portion becomes reddened by the acid and that the action of the acid gradually extends upwards till the whole of the liquid is red, in spite of the considerable difference in density of the acid and the litmus solution.

These experiments illustrate the diffusive power of fluid bodies, a power only possessed by solids to an extremely limited extent. That solids do diffuse, at a rate which is very small indeed compared with that at which liquids and gases intermingle, seems to be shown by such phenomena as the absorption of charcoal (carbon) by hot iron when the carbon is closely packed around it, with the consequent formation of steel, and the finding of appreciable traces of gold in the upper layers of a leaden bar to the top of which a piece of gold has been applied under pressure for some years.

45. When scientists discover such interesting facts as those we have illustrated in the experiments on diffusion, they try to form an idea of the probable cause of the observed phenomena. ("Phenomenon" simply means an "appearance", i.e. something seen or observed.) This generally involves the making of some supposition or assumption with regard to the nature of the bodies under examination and such an assumption is termed a "hypothesis". Hypotheses of this character are usually incapable of direct experimental proof, but are frequently of the greatest service in helping us to form a mind-picture of what may be going on during an experiment, and are further of value in that their consideration may suggest that other properties, hitherto unsuspected, are possessed by the substances dealt with. The existence or otherwise of such properties may be tested by further 
experiments, thereby widening our general knowledge, and, should new facts be discovered by this means, our belief in the stability of the hypothesis becomes more assured.

The hypothesis framed to account for such phenomena as the diffusion of fluid bodies is termed the "Kinetic Molecular Hypothesis". In this it is assumed that each form of matter consists of extremely minute particles called " molecules" (little masses). In solids, these are supposed to be comparatively closely packed, yet still to be separated from one another by distances which are large when compared with the space occupied by the matter in the molecule ; in liquids, the packing is usually less close; while in gases the molecules are very widely separated. Further, each molecule is supposed to be in a state of constant movement.

The movements of the molecules of a solid substance are of a comparatively restricted character. They are supposed to travel in limited paths around certain definite points in the solid and they are never far distant from these points. A mutual attractive forcetermed cohesion-exists between the molecules and prevents them from separating from one another except under the action of a considerable external force. A solid body consequently offers considerable resistance to change of shape.

In a liquid substance the molecules have partially lost their cohesive power, although this is still quite appreciable and prevents the volume of a liquid body from being readily affected by the application of external force. The freedom of movement of the molecules is, however, more pronounced than is the case with those of solids. They interchange positions without difficulty, sliding over and around one another, 
and their movements are not confined to any particular neighbourhood. The shape of a given mass of liquid is therefore easily altered by external agencies.

The molecules of gaseous substances have almost entirely lost the property of cohesion. They are supposed to move in straight lines with great rapidity, colliding frequently with one another and the walls of the containing vessel. The motion of any given molecule is practically unaffected by the presence of adjacent molecules. Their great freedom of motion in all directions carries them into all parts of any containing vessel, however large, the lack of cohesion between them permitting of their ready separation from one another.

Let us now apply this hypothesis to the explanation of what has been observed in studying gaseous diffusion. The molecules of bromine and those of the various constituents of the air being in a state of constant movement in all directions as described above, some molecules of bromine, by virtue of this motion; will leave the topmost layer of vapour and mingle with the molecules in the layer of air immediately above. On the other hand, some of the molecules in the lowest layer of air will leave that region and pass into that part of the jar formerly occupied by bromine. This process of intermingling will be comparatively slow, in spite of the rapidity with which the molecules move, for the collisions of the latter with one another and with the inside of the jar will cause them to be deflected into new paths with great frequency, and thus only part of the time will be occupied in moving in directions which will carry them into new regions. If a sufficient interval of time be allowed to elapse, however, the continuance of the intermingling process will at length 
result in the complete admixture of the bromine and air with one another, a result which our observations have shown us is actually achieved, although the density of bromine is more than 5 times that of air.

A precisely similar explanation of the phenomenon of liquid diffusion is afforded by the Kinetic Hypothesis.

Exercise for Student.

Suggest two reasons, based on the Kinetic Hypothesis, for gaseous diffusion being a more rapid process than liquid diffusion.

The above is naturally a very incomplete presentation of the Kinetic Hypothesis, and one other point deserves attention. The molecules of solids, liquids, and gases are not assumed to be all of similar size and mass. Thus the molecules of water are supposed to be larger than those of steam (= gaseous water) and we have some evidence that they are 3 to 4 times as large. On the other hand, the molecules of water are probably considerably smaller than those of ice $(=$ solid water).

46. Many interesting experiments may be performed which lend support to the idea of the existence in gaseous substances of molecules moving at rates which depend upon the density of the gas, the slower molecules being those of the heavier gases. One such experiment will next be described.

Experiment 26. Procure a glass tube about $18^{\prime \prime}$ long and of $\frac{1}{2}$ " bore. Make a cream of plaster of Paris and water in a shallow vessel, such as a saucer, and dip the end of the tube into this cream to a depth of about $\frac{1}{4}$. Fix the tube in an upright position with its end still in the cream, by means of a clamp and retort stand, and leave it till the plaster has set. Remove it and carefully cut away the excess of plaster 
from the end of the tube, leaving a plug about $\frac{1}{4}$ thick. . Cover this end of the tube, clamped in an upright position, with a large test-tube. Pass a piece of narrow indiarubber tubing, connected to the gas-supply, up the tube till the end of the rubber tube is nearly in contact with the plaster plug. Turn the gas-cock full on and allow the gas to pass for half a minute. Very slowly withdraw the rubber tube, and, when the open end of it reaches the lower end of the glass tube, close this end of the latter with the thumb. Turn off the gas and lower the end of the glass tube, still closed by the thumb, into a vessel of water coloured with litmus. Remove the thumb under water and clamp the glass tube in a vertical position with its lower end about $\frac{1}{2}$ " below the surface of the water. Remove the test-tube from the upper end. After a few minutes the water will be found to have risen in the tube above the level of that outside the tube and this rise will continue for some time, the water inside the tube sometimes reaching a point several inches above the level of the water in the outer vessel. Then a gradual fall occurs until the water-levels inside and outside the tube are the same.

As far as the eye can judge, the plaster of Paris plug is a continuous solid substance, but it really consists of innumerable very small particles separated by minute channels. The molecules of the constituents of the coal-gas inside the tube and of the air outside can traverse these channels readily, and do so, but at different rates. The molecules of the less dense constituents of the coal-gas escape from the tube through the plug much more rapidly than the air molecules pass inward. The result is that the total quantity of gaseous matter inside the tube is lessened and its pressure on the water-surface (see Chapter vI) decreased 
in proportion, so that the pressure of the atmosphere on the surface of the water in the outer vessel, which pressure remains practically unaltered throughout the experiment, is able to overcome that on the water inside the tube and thus forces some of the water from the vessel into the tube.

\section{Exercises for Student.}

1. Try to discover the reason for covering the top of the tube while it was being filled with coal-gas.

2. Assuming the Kinetic Hypothesis, account for the gradual fall of the water inside the tube after the maximum height reached is attained.

47. In the earlier part of this chapter, three different states of matter, solid, liquid, and gaseous, were considered and their more important properties dealt with. It must not be inferred that all kinds of matter may be classified rigidly under these three heads. In scientific work much classification is done, with a view to rendering large numbers of individual facts - seemingly isolatedeasier to grasp, and many definitions are framed, in order to give clearness and exactness to the various ideas concerned. The reader must be warned, however, that, although the boundaries of classes may be made as definite as possible and the definitions worded as clearly as may be, it is rarely the case that no overlapping occurs in classification or that a definition covers completely every example that may come within its terms. Thus, although our arrangement of bodies into the three groups above named is exceedingly convenient, any given body does not of necessity fall into one of those groups. Putty and pitch would probably be classified as solids from a cursory examination, since one can handle lumps of these substances without requiring a containing vessel. Yet, when left in a vessel for a sufficient length of time, they generally assume the shape of its interior and present a horizontal free surface, in this behaviour resembling liquids. Again, an experiment may be arranged in which a liquid is heated in a stout glass tube under pressure. At a certain temperature and under a certain pressure, H.D. S. 
which are known as the "critical" temperature and pressure of the liquid, it no longer presents a distinct free surface denoting the boundary between liquid and vapour, and the whole tube is filled with a substance which is in an intermediate state between the liquid and gaseous states, known as the "critical state". Any reduction of either pressure or temperature causes the immediate appearance of both liquid and gas simultaneously in the tube, as shown by the formation of two distinct layers separated by the horizontal free surface of the liquid.

\section{QUESTIONS}

1. Why is the smell of coal-gas frequently noticeable in all parts of a room in which there is a very small leak in the gas-fittings ?

2. Carbon dioxide is 22 times as dense as hydrogen. A mixture of equal volumes of these two gases is passed slowly along the stem of a long clay pipe and the gas issuing from the end is collected. What change will have been effected in the composition of the mixture by this treatment? Give reasons for your answer.

3. Why do you consider glass to be a solid and treacle to be a liquid?

\section{CHAPTER VI}

\section{FLUID PRESSURE}

48. In Chapter III it was stated that the force of gravitational attraction is universal and that the existence of this force is made evident to our senses by the behaviour of a body left unsupported in the air. The force with which the body and the Earth 
mutually attract one another causes them to move towards each other, the motion of the unsupported body being readily followed by the eye. A balloon, however, although unsupported by any visible means, does not fall to the Earth's surface, but even moves during part of its flight in the opposite direction. Similarly a cork dropped into water does not fall to the bottom of the liquid. In these cases a force is brought into play which is strong enough to overcome the force of gravity, and the consideration of the nature and magnitude of this force-exerted in common by all fluids - such as the air and the water in the examples above quoted-will form the subject of this chapter.

49. The way in which a fluid substance makes its presence felt in the case of a body in contact with it is by exerting what is termed a pressure upon all parts of the surface of the body which touch the fluid. When a $56 \mathrm{lb}$. weight is placed upon the ground, that portion of the ground upon which it rests is said to be under a pressure of $56 \mathrm{lb}$. If the area of the base of the weight be 28 sq. in., each sq. in. of the ground beneath it supports $\frac{56}{28}=2 \mathrm{lb}$. weight. In this case we call the pressure one of " $2 \mathrm{lb}$. per sq. in." and similarly we generally measure pressure by the magnitude of the force exerted upon unit area. We will next investigate the nature of the pressure exerted by fluids, by means of some simple experiments.

Experiment 27. Remove the brass plate from a "deflagrating spoon". Measure its diameter in $\mathrm{cm}$. and calculate its area. Pass a length of fine string through the hole in the centre of the plate and push in a tight-fitting cork, so that the string is held firmly and the arrangement is watertight. Weigh the disc, 
cork, and string. Place the disc on one end of a cylindrical Argand lamp chimney with the string inside the chimney, and hold the disc firmly against the end of the chimney by means of the string. Lower the chimney, disc end downwards, into a deep vessel of water, as shown in Fig. 25. When the disc is some inches below the surface of the water, release the string. It will be found that the disc does not fall from the end of thechimney. (If it does, the end of the chimney does not fit the

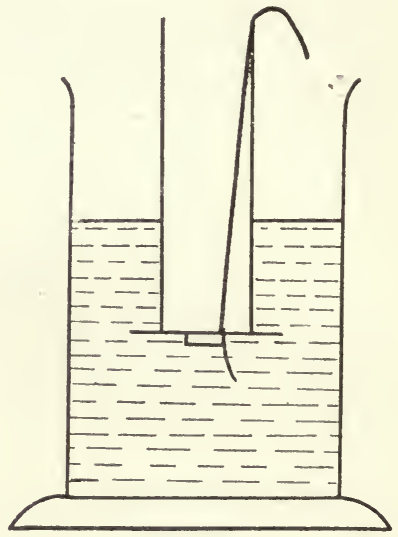

Fig. 25. upper surface of the disc tightly enough to form a watertight seal. In this case, the end of the chimney requires grinding to a flat surface by rubbing it, with the chimney in a vertical position, upon the ground glass surface of one of the plates used to cover gas-jars, a little fine emery powder mixed with oil being used as a grinding medium.)

Evidently a force is acting upwards upon the lower surface of the disc, pressing it against the end of the chimney, and overcoming the force of gravity which otherwise would cause the disc to fall. Carefully raise the chimney, with the string still loose, and it will be found that, at a certain point, the disc falls off, this being evidently due to the force of gravity being now slightly greater than the upward force. Note the position of the lower end of the chimney when this occurs, and measure the depth the end is then immersed 
below the water-surface. The magnitude of the upward pressure-usually called the " upthrust "- at this depth is found by obtaining the quotient of the weight of the disc, cork, and string previously found by the area of the disc. Calculate this and express your result as a pressure in grams per sq. $\mathrm{cm}$.

\section{Exercise for Student.}

What evidence did you obtain while performing this experiment of the existence of this upward pressure ?

Remove the chimney and disc from the water, dry them as completely as possible, and replace the disc on the end of the chimney. On the upper surface of the disc place weights equivalent to the weight of the disc, etc., as already determined, and again lower the arrangement to the bottom of the vessel. Loosen the string and slowly raise the chimney and its contents till the plate again drops off. Measure the depth of the end of the chimney below the surface of the water when this happens. Calculate the pressure at this depth.

On comparing the second depth with that first obtained, it will probably be found that the second is practically double the first. Since the pressure in the second case is also double that in the first, we are led to the conclusion that the upthrust varies directly with the depth of immersion. This conclusion should be tested by repeating the experiment, placing different weights on the upper surface of the brass disc, and noting whether the depth at which the disc falls off agrees with that required by the above statement.

50. To obtain some further information with respect to fluid pressure, measure the external diameter of the 
lamp chimney, and calculate the volume of the portion of the chimney immersed when the plate falls off in each determination made. This volume also represents approximately that of the water displaced by the apparatus at the instant the plate falls, if we neglect the amount displaced by the cork and disc. Since 1 c.c. of water weighs 1 gram, the weight of water displaced in each case is easily found. Compare these weights with the total weight of the disc, etc. used in the corresponding determination, and it will be noticed that, allowing for errors of experiment, the weight of water displaced is equal to the total pressure in each case.

For the above experiment there can be no pressure of the water in an up and down direction on the vertical walls of the lamp chimney. The whole upward pressure of the water on the immersed body is therefore that which we have measured on the horizontal disc. The results which we have found may be proved to be true in all cases, namely, that:

(a) The pressure on a surface immersed in a fluid varies directly with the depth of immersion;

(b) The total upward pressure of a fluid on an immersed body of any shape is equal to the weight-of the fluid displaced by the body.

The cylinder closed with a disc has been chosen for experiment because it has only vertical sides and a bottom surface; in the case of a fully immersed body with a top surface also under water, the total upward pressure is the difference between the upward pressure on the bottom surface and the downward pressure on the top surface. Since the top surface is at a less depth than the bottom surface, by $(a)$ above the downward pressure on the top is less than the upward 
pressure on the bottom; and the difference between them is equal to the weight of the water displaced.

\section{Exercise for Student.}

Show that the last statement is true in the case of a cube displacing 1000 c.c. whose top surface is $10 \mathrm{~cm}$. below the surface of the water. Calculate first the weight of water supported by the top surface; then, from $(a)$ above, deduce the upward pressure on the bottom surface.

51. A further and very important point concerning the nature of fluid pressure is that it is exerted equally in all directions. It is not an easy matter to arrange

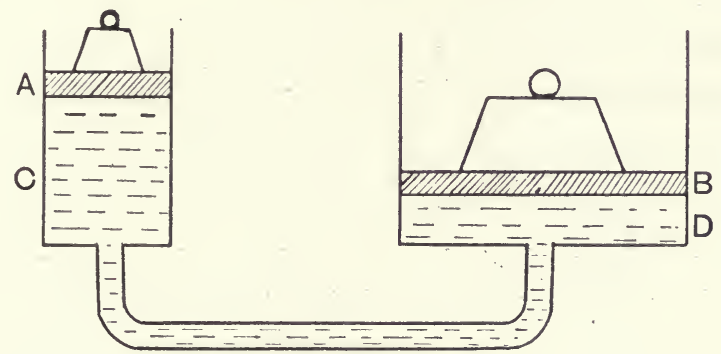

Fig. 26.

a simple experiment whereby this equality of pressure may be shown by actual measurement, but reference may be made to well-known facts in support of this idea. Thus we know that, if a rubber ball be squeezed between the thumb and forefinger, the whole of the exterior surface of the ball becomes harder to the touch. Compressing the ball at two points has decreased the volume, and hence increased the pressure, of the air within the ball. The new pressure, instead of being exerted only at the points of squeezing, is distributed evenly over the entire surface of the ball. An application of this principle is afforded by the hydraulic press, 
a diagrammatic representation of which is shown in Fig. 26. $A$ and $B$ are watertight pistons of different diameters which slide freely in the tubes $C$ and $D$. The entire space between the under surfaces of the two pistons is filled with water. The weights of the two pistons are so arranged that, when no extra load is placed on either piston, the lower surfaces of the pistons are at the same level. If now a weight of $1 \mathrm{lb}$. be placed on the top of $A$, this will cause increased downward pressure on the water-surface in contact with it, and, unless a corresponding increase in the upward pressure of the fluid on $A$ is effected, it is found that the piston $A$ will move downwards and $B$ will move upwards. That is to say, a downward pressure below $A$ has been converted into an upward pressure on $B$. Weights may now be placed on top of $B$ in order to balance the upward pressure on it. It will be noted, however, that a weight of $1 \mathrm{lb}$. is not sufficient to effect a balance, but that a considerably greater weight must be used in order to bring the apparatus to rest. Thus, suppose that the area of the lower surface of $A$ is $10 \mathrm{sq} . \mathrm{cm}$., while that of $B$ is $2000 \mathrm{sq} . \mathrm{cm}$., it is found that a.weight of $200 \mathrm{lbs}$. must be placed on $B$ to equalise the pressure of $1 \mathrm{lb}$. on $A$.

\section{Exercise for Student.}

Using the definition of pressure given previously, try to explain why the weight on $B$ must be $200 \mathrm{lb}$.

52. We will next attempt some experiments in which the effect of the upthrust on solid bodies denser than water, when they are immersed in that liquid, is considered.

Experiment 28. Obtain two bodies of equal volume, made of different materials, e.g. two cylinders 
of brass and iron respectively. Weigh each cylinder carefully, having previously attached a length of about $12^{\prime \prime}$ of stout thread to each. Over the lefthand pan of the balance place a bridge-a flat board supported on two blocks of wood of equal height will do-of such a height that the pan may move freely beneath it (this bridge is shown in Fig. 27). On the bridge place a vessel of water large enough to hold the cylinders without danger of their coming in contact with the inner wall of the vessel. Suspend each cylinder in turn from the stirrup at the left-hand end of the beam by means of the thread, and weigh each body when entirely submerged in the water and out of contact with the vessel. The upthrust on the cylinders will cause their weights, when in the liquid, to appear to be less than when they were weighed in the air. Note and compare the apparent losses of weight.

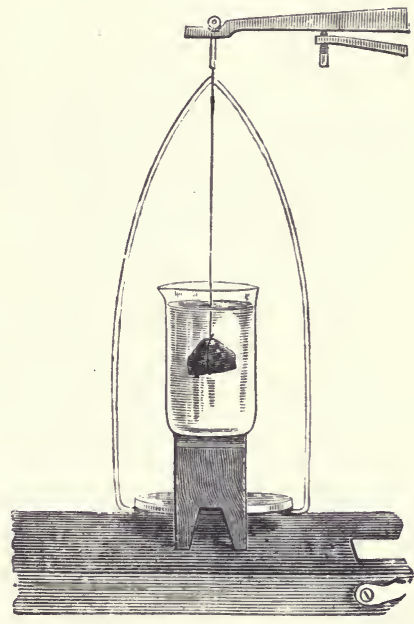

Fig. 27.

Repeat these operations with another cylinder made of one of the materials previously used, but of different volume.

The apparent loss of weight in each case is caused by the upthrust of the liquid on the solid. Determine the volume of each body used and hence find the weight of the water displaced by it. Compare the weight of the displaced water in each case with the corresponding 
apparent loss of weight of the solid body. If the experiment has been carefully performed, these quantities should be equal.

The result of this experiment is put into the form of a general statement as follows :

The apparent loss of weight suffered by a body as the result of immersion in a liquid is equal to the weight of the liquid displaced by the immersed body.

The above statement is known as the "Principle of Archimedes", having been first enunciated by that philosopher.

\section{Exercise for Student.}

In the above experiment water was the liquid used. Devise and carry out another experiment whereby the validity of the Principle of Archimedes may be tested in the case of some other liquid, e.g. methylated spirit.

53. The above-named principle is of great service in making determinations of the value of many quantities which are not readily found with accuracy by other means. The volume and specific gravity of an irregular solid body may be found as shown in

Experiment 29. Find the weight of the solid, say a lump of marble, in the ordinary way. Determine its apparent loss of weight when under water, as described in Experiment 28. Since this loss is equal to the weight of the displaced water, the volume of the displaced water is easily found, and hence the volume of the body which displaced it.

The determination of the density, or of the specific gravity, of such an irregular solid only requires one further step to be made in the argument. Thus, to find the density of marble, the weight of the marble obtained in the above experiment has only to be divided by the volume found as shown. 


\section{Exercise for Student.}

Using the values found for the weight and volume of the lump - of marble in Experiment 29, write out fully the various steps of the argument by which you consider the specific gravity of marble may be obtained therefrom.

Experiment 30. Weigh a solid body, such as the glass stopper of a bottle, in air, in.water, and in another liquid-say paraffin oil. Note the apparent loss of weight of the stopper when weighed in the water and also in the paraffin. Apply the Principle of Archimedes to the problem of finding the specific gravity of the paraffin oil from the measurements made.

54. The Principle of Archimedes does not apply directly to the case of bodies floating in a liquid, since the weight of any such body is entirely supported by the upthrust of the liquid and consequently it appears to lose all its weight under these conditions, whatever its volume may be. A similar principle may be deduced as the result of a simple experiment.

Experiment 31. Procure a flat-bottomed tube, such as is commonly used for the preservation of chemical specimens, and pour into it small leaden shot in sufficient quantity to keep it, when it is floated in water, in an upright position. Cork the tube, and float it thus prepared, in a vessel of water. Note carefully the height to which the liquid rises on the outside of the tube. Remove the tube from the water and dry it. Find by calculation from the external dimensions of the part of the tube which was under water, the volume, and hence the weight, of water displaced by the immersed portion. Weigh the tube and shot, and compare this weight with that of the water displaced by the apparatus.

Repeat the operation with a larger quantity of shot 
in the tube so that it floats with a larger portion of its volume immersed. Find the weight of displaced water and the weight of the dry tube and shot as before. Again compare these weights.

It will be found that the weight of the solid body is equal to that of the volume of water displaced by it. Put into the form of a general statement this is known as the "Principle of Floating Bodies", which is as follows :

The weight of the liquid displaced by a body floating in a liquid is equal to the weight of the body.

\section{Exercise for Student.}

Devise and carry out an experiment to test the applicability of the Principle of Floating Bodies to a liquid other than water.

55. Instruments have been devised whereby advantage may be taken of the above principle to determine the specific gravity of a liquid in a very simple manner. They are termed "hydrometers", and Fig. 28 shows the appearance of one of the ordinary forms. $B$ is a long cylindrical bulb of glass. $C$ is a smaller bulb sealed to the lower end of $B$, and containing a quantity of small leaden shot or some mercury. $A$ is a long stem of narrow bore, in which is a paper scale. This scale is so graduated that the specific gravity of any liquid, in which the hydrometer will float at such a depth that the level of the liquid is at some point on the stem, may be read directly from the scale. Hydrometer scales usually have a limited range in order to secure a greater degree of accuracy in the measurement obtained and to do away with the great length of

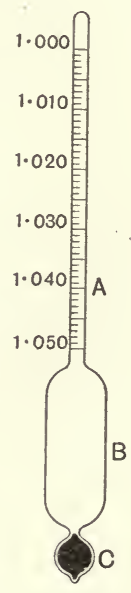

Fig. 28. 
stem which would be necessary to provide for the inclusion of the greatest and least values likely to be met with, while at the same time giving readings sufficiently exact for general purposes. Thus a set of hydrometers with the following ranges would be capable of utilisation for finding the-specific gravity of most liquids :

$0.700-0.800 ; 0.800-0.900 ; 0.900-1.000 ; 1.000-$ $1 \cdot 200 ; 1 \cdot 200-1 \cdot 400 ; 1.400-1 \cdot 600 ; 1 \cdot 600-2.000$.

As an example of the use of one of these instruments, we will describe the determination of the specific gravity of salt solution in

Expertment 32. Make a $10 \%$ solution of common salt by dissolving $50 \mathrm{~g}$. of salt in water and making the total volume of the solution up to 500 c.c. ${ }^{1}$ Pour this liquid into a tall glass jar. Select a hydrometer with a range from $1 \cdot 000$ to $1 \cdot 100$ or $1 \cdot 200$. Float this in the salt solution, taking care that it floats freely, out of contact with the inner wall of the jar. Examine the part under the liquid to see that no air-bubbles are clinging to the hydrometer. Remove any that are noticed with a long-handled camel-hair brush. When the hydrometer is floating steadily, take the reading at the level of the liquid. It will be noted that the liquid creeps up the stem, forming a curved surface similar to that assumed in the burette and other measuring vessels. Allowance must be made for this effect of capillary attraction by adding a correction-usually onehalf the value of a single scale-division-to the reading taken at the point where the liquid surface appears actually to meet the stem. Or, if preferred, the reading

${ }^{1}$ Strictly, $50 \mathrm{~g}$. of salt should be dissolved in $450 \mathrm{~g}$. of water. The above is, however, the conventional laboratory method, and will be adopted throughout this book. 
may be taken in a jar of such a size that, when the hydrometer is floating in the liquid, the liquid surface is at the top of the jar, and the reading at the general level of the liquid may be taken through the meniscus. This is not very easy to do with accuracy and cannot, of course, be done in the case of an opaque liquid, and the allowance of half a scale-division is sufficiently exact for all practical purposes. Test the accuracy of the graduation of the hydrometer by finding whether, when floated in distilled water, the scale-reading is exactly 1.000. A further check may be made by finding the specific gravity of the salt solution by a different method, such as by the use of a density flask, and noting whether the two results agree.

\section{Exercise for Student.}

Prepare a solution of salt with a specific gravity of 1.010 by means of the hydrometer.

56. The lactometer is simply a form of hydrometer in which the scale has a range which makes the instrument suitable for finding the specific gravity of milk. A common range is from $1 \cdot 015$ to $1 \cdot 040$, and the markings on the scale usually consist of the last two figures only of the specific gravity, the 1.0 being understood as to be added to all readings taken with the instrument. Thus a reading of 32.5 on the scale would show a milk of specific gravity $1 \cdot 0325$. The value of the information obtained by means of the lactometer in judging of the genuine character of the sample of milk tested will be treated fully in Part II.

57. In the statement of the Principle of Archimedes already given, the case of a body immersed in a liquid alone receives recognition. It has been previously mentioned that all fluids-both liquid and gaseous - exert pressure on bodies in contact with them. 
From this similarity of behaviour of liquids and gases it is to be expected that bodies weighed in a gas also suffer an apparent loss of weight, this loss being caused by the partial support of the body by the pressure of the gas. This loss actually takes place, and its magnitude may be found by determining the weight of the body in a vacuum and also its weight when weighed in an atmosphere of the gas. This experiment is rather beyond the scope of our work, but, as a result of carefully carried out experiments, it has been shown that the Principle of Archimedes holds good for gases as well as liquids.

\section{Exercise for Student.}

Frame a statement of the Principle of Archimedes which shall include the case of gases as well as that of liquids.

All weighings are ordinarily carried out in aira gas-and, as a consequence, the weights obtained are slightly smaller than the actual weights of the bodies concerned. By "actual weight" is intended the total pull of the Earth upon the body weighed. The weight of a litre of air is about 1.293 grams. Since the loss of weight is equal to the weight of displaced gas, it is easy to calculate the error in the weight of a body weighed in air, if the volume of the body be known.

\section{Exercise for Student.}

Why are the weights of the Standard Pound and Kilogram defined as obtained in a vacuum?

The error involved in weighing the comparatively small bodies dealt with in our work, due to the upthrust of the air, is negligible when compared with other sources of error in the weighings. It must be remembered, however, that, in all scientific work of the highest character, allowance is always made for the errors in 
weighing in air due to the volumes of air displaced by both the objects weighed and the weights used.

58. We live in a gaseous atmosphere and as a result are under pressure due to the weight of that atmosphere every moment of our lives. That we.are not ordinarily conscious of this pressure is explained by the fact that our bodily structure is specially adapted for existence under the average fluid pressure of the air. The troubles of balloonists and mountain-climbers at high altitudes, as well as those of divers in a divingbell and workers in the caissons used in tunnelling, are caused by the great differences of air-pressure that they are called upon to experience. The magnitude of the pressure of the air is generally measured by methods illustrated in the succeeding experiments.

Experiment 33. Obtain three glass tubes, closed at one end, and about $36^{\prime \prime}$ in length. Fill the first with water, put the forefinger over the open end, and invert the tube with its end under the surface of some water in a beaker or other suitable vessel. Remove the finger and clamp the tube in an upright position with its open end still under the water-surface. Similarly fill a second tube with glycerine and invert it in a vessel containing that liquid. Fill the third tube with mercury and invert it in a shallow bowl of mercury. It will be noticed that the tubes containing water and glycerine remain completely filled with liquid, while in the case of the mercury the tube is only partly filled with that liquid, the surface of the mercury in the tube standing about 30 inches higher than the level of the liquid in the outer vessel.

It is evident that the weight of the liquid in each tube tends to cause the liquid to fall out of the tube into the vessel below. The force which counterbalances 
this tendency in each case is the pressure of the air on the surface of the liquid in the outer vessel. This pressure is transmitted evenly throughout the liquid in all directions, as has been previously explained, and at the point in the tube at the same level as the surface of the liquid in the vessel, we have the two forcesthe weight of the liquid in the tube acting downwards and the transmitted pressure of the air acting upwardsexactly counteracting each other's effects. That this is the true explanation of this phenomenon was tested by Pascal. He caused a tube filled with mercury and inverted as above to be carried from the foot to the top of the Puy de Dôme, one of the lofty peaks of the Auvergne Mountains. He argued that, if the column of mercury was really supported by the pressure of the superincumbent air, the height of the column supported at the top of the mountain should be less than that at the bottom, since there must be less air above the surface of the mercury in the outer vessel at the top of the mountain than at the bottom, and consequently it must possess less weight and exert less pressure. Great was his satisfaction when it was reported to him that the mercury behaved exactly as he had expected, the height of the column supported becoming gradually less as the apparatus was carried higher. We may not repeat this classic experiment with convenience, but we may easily investigate the effect of removing some of the air from the outer vessel by performing

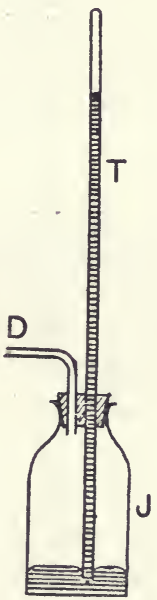

Fig. 29.

Experiment 34. Fit up a piece of apparatus similar to that illustrated in Fig. 29. $J$ is a wideH. D. S. 
mouthed bottle, fitted with a tight-fitting rubber stopper, bored with two holes. One of these holes admits a glass tube $T$, similar to those used in the preceding experiment, filled with, and inverted over, mercury in the bottle, and the other a short length of glass tubing $D$, bent at a right angle. The outer end of $D$ is attached to an air-pump-or to a water-pump as commonly used by chemists to hasten filtration. The level of the mercury in the tube is noted and the pump is gently worked. The height of the column of mercury in the tube will be seen to diminish gradually as the air is removed by the action of the pump from the vessel. This diminution will continue till the limit of the efficiency of the pump is attained, when the mercury will remain at a more or less constant level. Pinch the rubber connection to the pump tightly and disconnect the pump from the other end of the connection. Open the rubber tube by releasing the pressure of the fingers on it.

\section{Exercise for Student.}

Explain as clearly as you can why the mercury level in the tube changes during this experiment.

59. The original experiment with the tube of mercury is due to Torricelli and the experiment is often referred to as the "Torricellian experiment", while the apparatus is called the "Torricellian barometer" (Greek, baros, weight, and metron, measure). A barometer is then an instrument whereby the pressure of the atmosphere is measured by balancing it against the action of another force-the force in this case being the weight of the column of mercury. Other forms of this instrument exist, of which the $\mathrm{U}$-tube barometer, or siphon barometer, is the most commonly occurring 
example. This consists of a long glass tube bent round on itself so that one limb of the tube is much longer than the other. The longer limb is closed and is nearly full of mercury, which also occupies a portion of the shorter limb, as shown in the accompanying figure. The tube is filled with mercury in such a way that the space above the liquid in the long limb is empty, as is also the case with the Torricellian form of the instrument, this empty space being generally known as the "Torricellian vacuum". Thus the surface of the mercury in the long arm of the barometer is under no pressure save that of the exceedingly small quantity of mercury vapour which volatilises into the space at the top of the tube. On the other hand, the free surface of the mercury in the short limb is subject to the pressure of the atmosphere. The distance between the levels of the liquid measures the magnitude of the air-pressure and is found to vary from day to day. To enable this distance to be measured, a scale of centimetres and millimetres is etched on the short limb and on the upper part of the long one. Both scales are numbered from a zero

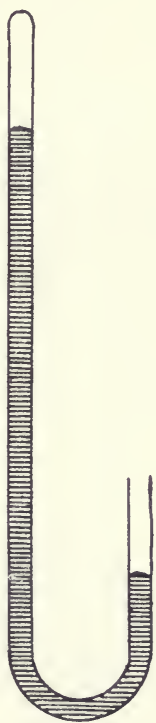

Fig. 30 . point about midway between them, the numbers on the scale on the long arm reading upwards and those on the other arm downwards. Thus the height of the mercury column supported by the pressure of the atmosphere is the sum of the readings taken at the levels of the mercury. 
Exercise for Student.

Why is it necessary to have two scales on which to take readings ?

60. In the more elaborate instruments, such as Fortin's standard barometer, arrangements are made whereby the variations of level of the surface of the mercury exposed to the air, due to the changes in the atmospheric pressures, are compensated for by bringing this surface back to a definite position before readings are taken. Thus only one scale is required situated beside the upper level of the mercury.

Hall barometers are commonly of the U-tube type, but are provided with simple mechanism, actuated by the fluctuations in level of the mercury in the open limb, whereby a long pointer is made to travel round a circular scale, graduated in inches and decimal fractions of an inch. By this means readings of the height of the mercury column may be made directly from the circular scale.

61. A barometer of quite a different type is the "Aneroid". In this form the variations of air-pressure are utilised to alter the volume of a box made of metal, with a thin corrugated lid. The air having been removed from the metal box, the lid is kept from collapsing by a strong spring; the movements of the lid of the box inwards or outwards, corresponding to increase or diminution in the pressure of the atmosphere, cause the end of a long pointer to travel round a circular scale similar to that described above. Since the movements of the lid are extremely small, a system of multiplying levers is made use of to render them readily measurable. These instruments are made of such delicacy as to show distinctly the difference of pressure due to carrying the barometer up a short 


\section{Fluid Prêssurés:}

flight of stairs. On this account, and because of their ready portability, they are much used in determining the heights of mountains or of balloon ascents. When used for this purpose, a scale of heights is frequently found upon them, in addition to the ordinary scale of pressures. A decrease in pressure of $1^{\prime \prime}$ of mercury is approximately equivalent to an increase of altitude of about 900 feet.

The dials of aneroid and hall barometers are frequently marked at each half-inch, beginning at 28 inches, with the words Stormy, Much rain, Rain, Change, Fair, and Set fair. The weather predictions thus afforded are at best only approximate. They are the result of long series of observations upon the behaviour of the barometer during spells of weather of various kinds. Thus it has been noted that, in our latitudes, the barometer usually falls when the wind is in the S.W., the quarter from which most of the rain which falls in this country may be expected. On the contrary, a N.E. wind in general causes a rise of the barometer, accompanied by fine weather.

In a later chapter, the question of the quantity of water-vapour present in the air will be further dealt with. For the present, it may be mentioned that aqueous vapour is less dense than air and a column of air containing much vapour is lighter, and consequently exerts less pressure, than a column of drier air of equal height. Warm air is also less dense than cold air, and it is commonly the case in this country that damp air is warmer than dry. Hence the barometer registers lower pressures when moisture is contained in large proportion in the air, and this is an atmospheric condition conducive to the production of rain.

It must be remembered that the height of the place 
of observation has a considerable effect upon the reading obtained and the predictions which may serve with fair accuracy for low-lying stations, may be entirely misleading for others at greater-altitudes.

62. In most of the instruments and experiments described in this chapter, the pressure of the air has been balanced against that due to a column of mercury. A classical experiment, due to Robert Boyle, in which pressures are measured by the same means, will give us valuable information upon another point, namely, the influence of pressure upon the volume of a gas.

Experiment 35. Close one end of a piece of glass tubing, about 50 inches long, and with a bore of at least $1^{\prime \prime}$. Bend the tube round on itself at a distance of about $10^{\prime \prime}$ from the closed end, so that its appearance is as shown in Fig. 31. Clamp the tube in an upright position. Insert a small funnel into the upper end of the long limb, and carefully pour in mercury till the liquid closes the bottom of both tubes and is at the same level in each. The air in the short limb is thus cut off from the outside atmosphere. The pressures on the free surfaces of the mercury must be equal, since the mercury is at the same level in both arms of the tube, and is at rest. The pressure on the mercury surface in the long limb is evidently that of the atmosphere. Measure this pressure by means of a barometer. Measure

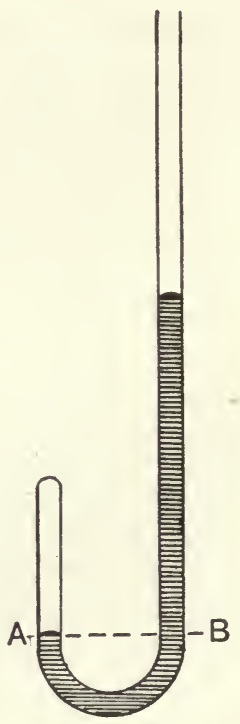

Fig. 31. the length of the column of air contained in the 
short limb. If we assume the bore of the tube to be uniform, the volume of the contained air will be proportional to the length of the portion of the tube containing it. The enclosed volume of air in the short limb is then under a pressure equal to that of the surrounding atmosphere and has a volume proportional to the length of tube measured.

We -will now alter the pressure of this air. Pour a little more mercury into the long limb. When the mercury in both limbs is at rest, it will be noticed that the level of that in the longer limb is higher than that in the other. (See Fig. 31.) Let us now consider the pressures acting at the level shown by the dotted line $A B$ in the figure. The only pressure acting downwards at this level in the closed limb is that of the contained air. At the same level in the other limb we have the pressure of the atmosphere acting downwards upon the free surface of the mercury, and transmitted through that liquid in all directions, together with the pressure of the column of mercury in the tube above the level $A B$. These two pressures combined must be equivalent to the pressure of the air in the other limb, since the pressures of the two columns of mercury below $A B$ balance one another, and hence need not be considered. The pressure of the air in the closed limb is therefore the sum of the atmospheric pressure and the height of the mercury surface in the long tube above $A B$. Measure this height and also the length of the air column in the short tube under the new pressure.

Add more mercury, and again measure the length of the contained air column and the height of the mercury surface in the long tube above that in the shorter. Continue the addition of mercury in small 
quantities, noting the pressure and length of the air column in the closed limb after each such addition.

Tabulate your results as shown below and find for each pressure measured the product of the pressure and volume of the air in the closed limb.

Let us suppose that the following are the observations made in such an experiment as the above:

\begin{tabular}{|c|c|c|c|c|}
\hline $\begin{array}{c}\text { 1. Pressure } \\
\text { of } \\
\text { Atmosphere }\end{array}$ & $\begin{array}{l}\text { 2. Distance } \\
\text { between } \\
\text { mercury levels }\end{array}$ & $\begin{array}{c}\text { Sum of } 1 \\
\text { and } 2 \\
(P)\end{array}$ & $\begin{array}{l}\text { Length } \\
\text { of air- } \\
\text { column }(V)\end{array}$ & $P \times V$ \\
\hline $750 \mathrm{~mm}$. & $0.0 \mathrm{~mm}$. & $750 \mathrm{~mm}$. & $20.0 \mathrm{~cm}$. & 15,000 \\
\hline , & $22.0 \mathrm{~mm}$. & $772 \mathrm{~mm}$. & $19.4 \mathrm{~cm}$. & 14,970 \\
\hline , & $46.0 \mathrm{~mm}$. & $796 \mathrm{~mm}$. & $18.9 \mathrm{~cm}$. & 15,040 \\
\hline , & $125^{\circ} 0 \mathrm{~mm}$. & $875 \mathrm{~mm}$. & $17 \cdot 1 \mathrm{~cm}$. & 14,960 \\
\hline 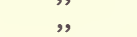 & $680^{\circ} 0 \mathrm{~mm}$. & $1430 \mathrm{~mm}$. & $10.4 \mathrm{~cm}$. & 14,870 \\
\hline
\end{tabular}

It will be seen that, allowing for errors of experiment, the value of the product $P V$ remains the same throughout the series of observations. Since $V$ is a measure of the volume of the air contained in the closed limb, and $P$ is the pressure, expressed in millimetres of mercury, to which that air is subjected, the general result of this experiment may be stated thus :

The product of the volume and the pressure of a given mass of gas is constant.

63. The above relation between the pressure and volume of a gas may be stated in another form. It will be noticed that, as the pressure is increased, the volume decreases, and it may readily be shown that doubling the pressure decreases the volume by one-half, trebling the pressure reduces the volume to one-third and so on. Put into the form of a general statement, this becomes:

The volume of a given mass of gas varies inversely with the pressure to which it is subjected. 
In honour of Robert Boyle, who first enunciated the above law, it is called "Boyle's Law"1. It is assumed that, during the performance of experiments such as that just described, neither heating nor cooling of the enclosed gas is allowed to take place, since, as will be demonstrated later, these changes of temperature will cause corresponding changes of volume independently of those caused by the variations of pressure, thus nullifying to some extent any deductions drawn from the observations made.

\section{Exercise for Student.}

Try to deduce the second statement of Boyle's Law given above from the observations quoted in the table.

64. The gas used in our experiment was air, the most easily procured, and therefore most convenient, gas for our purpose. Similar observations would have been obtained, however, had the gas enclosed in the tube used been any other of the commonly occurring gases. Thus, in the statements of the law given, the word "gas" is used, and not "air", in order to show the general applicability of the law to all gaseous substances.

It must not be inferred, however, that the law is strictly applicable to the behaviour of all gases under all conditions of temperature and pressure. An important deviation is noted in the case of some gases when they are submitted to considerable increase of pressure at the ordinary temperature, the decrease of volume corresponding to a given increment of pressure being distinctly greater than that required in accordance with

1 This law was independently discovered at a later date by the French investigator, Mariotte, and hence is generally referred to on the Continent as "Mariotte's Law". 
Boyle's Law. The substances which show this peculiarity are all obtainable in the liquid form at ordinary temperatures by being sufficiently compressed and, on cooling them to temperatures which are not far removed from the ordinary temperature, also assume the liquid form. Sulphur dioxide - a gas obtained by burning sulphur in air-is an example of this class. It becomes a liquid at $-10^{\circ} \mathrm{C}$. under atmospheric pressure, and is obtainable commercially in the liquid form, being stored in stout bottles resembling soda-water syphons. It is forced into these bottles under pressure and is retained in them as a liquid at all ordinary temperatures. Such a gas-readily liquefiable by cold, and capable of liquefaction at ordinary temperatures by pressure alone-is frequently termed a "vapour". On the other hand, nitrogen-one of the gases of the atmosphere-must be cooled to $-195^{\circ} \mathrm{C}$. under atmospheric pressure before becoming a liquid, and cannot be liquefied by increase of pressure alone at ordinary temperatures. At temperatures near its boiling-point, however, nitrogen exists in the vaporous condition, being then readily liquefiable by increase of pressure alone.

65. Problems dealing with the changes of volume of gases with change of pressure may be readily solved by such methods as that shown in the following example :

A room is $15 \mathrm{ft}$. long, $12 \mathrm{ft}$. wide, and $8 \mathrm{ft}$. 6 in. high. Find the volume of air which escapes from the room when the atmospheric pressure alters from $750 \mathrm{~mm}$. to $735 \mathrm{~mm}$.

The interior volume of the room, and hence that of the air contained in it, is $\left(15 \times 12 \times 8 \frac{1}{2}\right)=1530 \mathrm{cu}$. ft. The product of the numbers representing the volume 
and pressure of this mass of air is therefore $(1530 \times 750)$ $=1,147,500$, when the pressure is $750 \mathrm{~mm}$. This must also be the product of the volume and pressure when the latter is $735 \mathrm{~mm}$., according to our first statement of Boyle's Law. If we denote the volume when the pressure is $735 \mathrm{~mm}$. by $V$, we have $735 \mathrm{~V}=1,147,500$, whence $V=1561 \cdot 2 \mathrm{cu}$. ft. Thus the volume of air that escapes from the room is $(1561 \cdot 2-1530)=31 \cdot 2 \mathrm{cu}$. $\mathrm{ft}$.

A similar result is obtained by making use of the second form of statement of Boyle's Law. Thus we may find the volume of the room as above, and since the volume of the air contained in it will increase on account of the diminution of pressure, and we know that the increase of volume will be inversely proportional to the decreased pressure, we have

New volume of air $=(1530 \times 750) \div 735$;

i.e. $1,147,500 \div 735$, as before.

\section{QUESTIONS}

1. Why is it a somewhat difficult matter to keep a fully inflated football entirely under water?

2. The standard pressure of the atmosphere is equivalent to that of a column of mercury $760 \mathrm{~mm}$. in height. If the specific gravity of mercury is 13.6 , find the standard pressure of the atmosphere in grams per square centimetre, and in lbs. per square foot.

3. If a glass tumbler be completely filled with water, the mouth of the tumbler closed with a postcard held firmly against the edge of the mouth with the hand, and the tumbler then inverted, the hand may be removed without the water being spilt. How do you explain this phenomenon?

4. An egg weighs $61 \cdot 24 \mathrm{~g}$. When weighed in water, its apparent weight is $1 \cdot 46 \mathrm{~g}$. What is the average density of the constituents of the egg ? 
5. In a determination of the specific gravity of brine, the following observations were made :

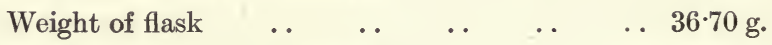

Find the specific gravity of the brine.

filled with water $\ldots \quad \ldots \quad \quad \ldots 8967 \mathrm{~g}$.

$\begin{array}{ccccccc}" & \quad & \text { filled with water } & \ldots & \ldots & \ldots & 89 \cdot 67 \mathrm{~g} . \\ & \# & & \end{array}$

6. The specific gravity of a sample of olive oil is 0.915 . What will be the apparent loss of weight of a brass cube of $3 \mathrm{~cm}$. edge when weighed in the oil ?

7. A lump of aluminium weighs $(a)$ in air, $30 \mathrm{~g} .,(b)$ in water, $18.4 \mathrm{~g} .,(c)$ in chloroform, $12.6 \mathrm{~g}$. Find the specific gravities of the metal and the chloroform.

8. How much air will enter a room $20 \mathrm{ft}$. long, $16 \mathrm{ft} .9 \mathrm{in}$. wide, and $10 \mathrm{ft}$. high, if the pressure of the air varies from $29.5 \mathrm{in}$. to $30.1 \mathrm{in}$., as measured by the barometer ?

9. A partially inflated bladder is placed inside a stout-walled glass flask, and the air is gradually exhausted from the flask by means of an air-pump. What would you expect to happen to the bladder? Give reasons for your opinion.

10. Why is it easier to float in sea-water than in fresh water ?

11. When the tube of a Torricellian barometer is gradually inclined from the perpendicular, the level of the mercury surface approaches the closed end of the tube until finally the tube is completely filled with the liquid. How do you account for this?

\section{CHAPTER VII}

\section{CENTRE OF GRAVITY}

66. When discussing the meaning of the terms "weight" and "gravity" in Chapter III, it was mentioned that the attraction between two bodies is increased when the distance between their centres of 
gravity is lessened. The words "centre of gravity", as used in this statement, require explanation. In the case of a hammer, consisting of an iron head attached to a wooden handle, the centre referred to would not be situated about halfway along the handle, but at a point in the head. This point, through which the total force of attraction exerted by the earth on the hammer and the hammer on the earth may be considered as acting, is called the "Centre of Gravity" of the hammer.

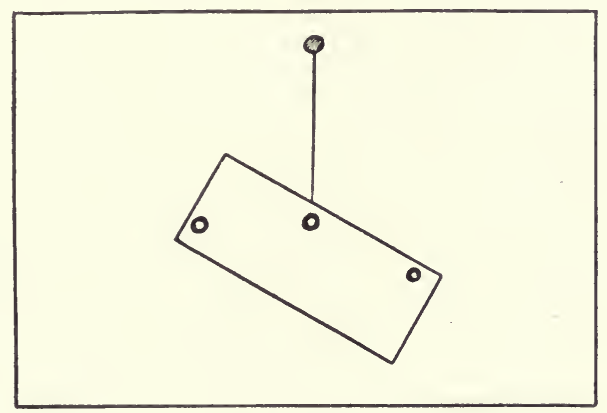

Fig. 32.

67. To elucidate further this idea of a centre of gravity some simple experiments may be performed in which its position is determined in the case of certain bodies.

Experiment 36. Cut out from millboard of good quality a rectangle, a parallelogram, a circle, and an irregular figure. These figures should not be too small - each should have an area of, say, 300 sq. cm. Bore three small holes in the surface of each figure at any points (see Fig. 32). Attach a short length of bent copper wire to a cotton thread to serve as a hook. 
Pass the hook through one of the holes in the rectangle, and tie the other end of the thread to a nail fixed in a drawing-board or other convenient wooden board. Support the board in a vertical position so that the rectangle hangs freely in front of it. Allow the figure to come to rest. Mark on the surface of the millboard the point at which the thread crosses the edge of the figure and draw with a straight-edged ruler a line from this point, through the centre of the hole, to the opposite edge of the figure. Repeat these operations with the hook in each of the other holes in turn. It will be noted that the three lines drawn all pass through one point, which in this case will be found to correspond with the point of intersection of the diagonals of the rectangle.

In the above experiment two forces are actingthe pull of the thread upwards and the weight of the millboard downwards. These two forces balance one another, since the rectangle remains at rest. Now two forces can only balance one another when their lines of action are in one and the same straight line. (This may be tested by displacing the rectangle, suspended as above, to one side, so that the line of action of the pull of the thread is no longer vertically upwards. On releasing the rectangle, so that it is acted upon by the two forces mentioned above, it will move from its position of displacement and, after swinging for a short time, will come to rest with the thread hanging in a vertical direction, i.e. in the same direction as that in which the force of gravity acts.) From the above considerations it is clear that the weight of the body acts in each case in directions which all pass through a certain point. As stated previously, this point is called the centre of gravity of the body. 
Test the accuracy of this statement in the case of the rectangle by making another hole in it and suspending it again with the hook through this hole. If what has been said is correct, the straight line drawn in the direction of the thread should intersect the lines already drawn, at the point previously determined.

Find, in a similar fashion, the centres of gravity of the other figures you have prepared.

The true centre of gravity of each figure will not, of course, be at the point of intersection of the lines drawn on the surface of the millboard, but will be situated at a point immediately beneath this point and halfway through the thickness of the millboard.

68. The centre of gravity of any body might be found by the method just described, if it were feasible to mark the exact direction of the lines of action of the weight of the body when it is suspended from different points on its surface. This cannot very well be done in most cases, however, but we may find the centre in the case of regular solids with some approximation to accuracy by adaptations of the above method.

Experiment 37. Balance a boxwood metre scale upon one edge of a wooden triangular prism, with the flat side of the scale upon the edge of the prism. When it rests in a horizontal position, its weight acting downwards must be acting in the same vertical plane as the resistance of the edge of the prism acting upwards. That is to say, its centre of gravity must be situated at some point in the interior of the scale which is exactly over the edge of the prism. Balance the scale again, this time upon one of its long narrow faces. It will be found that it balances about a point in its length which is situated at the same distance from either end of the scale as the point previously found. At some point in 
the vertical plane, containing the edge of the prism, above this position of rest is its centre of gravity. If it were possible to balance the scale upon one end, a third vertical plane could be found containing the centre of gravity. The three planes thus determined have only one common point of intersection, and this must be the centre of gravity of the scale. By making the assumption, however, that the middle part of the scale is composed of homogeneous matter, we may consider the centre to be midway along the portion of

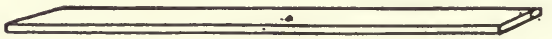

(a)

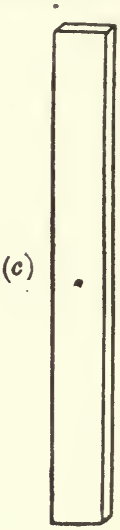

Fig. 33.

the line which forms the intersection of the two planes found by actual experiment, contained within the scale.

69. It will probably have been noted that it is a much more difficult operation to balance the scale upon one of its long narrow faces than upon one of the flat ones, and that the feat of balancing it upon one of its end faces seems impossible. We express the ideas derived from these observations by saying that, when supported upon a flat face, the scale is in a more stable position than when balanced about a narrow edge, and 
that the scale, when standing on end, is in a very unstable position. We will next consider the reason for these differences in behaviour of the scale. Fig. 33 shows such a long wooden lath as the scale already used resting on the ground on
(a) a flat face;
(b) a narrow long face;
(c) an end.

The approximate position of the centre of gravity of the lath is shown by a black dot. In each position of the lath, the centre of gravity, when the lath is at rest, is vertically over some point on the ground covered by the part of the lath in contact with the ground. It will be noticed, however, that the height of the centre above the ground is least when the lath is on one of its flat sides, and greatest when the lath is standing on end. Further, it is clear that the base of support of the lath is of greatest area when it is in position $(a)$, and least in position $(c)$. This leads to the idea that the stability or otherwise of a body is directly connected with the height of its centre of gravity above its base of support, and also with the area of the base of support. Suppose the lath standing on end to be displaced from this position. A very small displacement in any direction will cause the perpendicular from the centre of gravity to the ground-that is to say, the line of action of the weight of the lath-to fall on some part of the ground not included within the area of the portion of ground upon which the lath was supported when at rest. The lath, if unsupported in any way, will now fall, and come to rest with its centre of gravity nearer to the ground in one of the positions $(a)$ or $(b)$. This is due to the moment of the weight of the lath about the point or points at which it touches the 
ground. This moment has its point of application at the centre of gravity, and its effect is to cause the lath to be further displaced in the direction of the uriginal displacement. A body is said to be in a position of " unstable equilibrium " when a small displacement of the body from its original position of balance causes further displacement to occur, so that the body takes up a new position with its centre of gravity at a lesser distance from the base of support.

When the lath is poised about a long narrow face, a small angular displacement may not cause the line of action of its weight to fall without the base of support, and in this case it will be found to return to its original position when the disturbing force is removed. It is then said to be in a fairly stable position. Its position of greatest stability is, however, that in which it is supported on one of its long flat faces. This is the position it always takes up when disturbed from the positions already considered, if the disturbing force be sufficiently great. A body is said to be in "stable equilibrium " when, after a small angular displacement in its position has been effected under the action of a force, it returns to its original position.

As already illustrated above, there are degrees of stability, and this point may be further examined if we deal with the various positions in which an ordinary brick may be balanced. Its stability increases throughout the series of positions in which it may be balanced given in the following list :

(a) standing on one corner ;
(b)
,
(c)
(d)
(e)
of its shortest edges ;
,, longest edges ;
,, smallest faces;
, largest faces. 


\section{Exercise for Student.}

Which of the positions named above would you term "stable" and which " unstable" ? Make a further list of positions in which the brick may be balanced, classifying them under these heads. Which position do you consider that of greatest stability ?

70. There is yet another form of equilibrium to be considered. If a ball be placed on a horizontal surface, it will remain in any spot on which it is placed. If now it be displaced from this position by a small disturbing force, it neither returns to its former position, nor moves further from that position. Hence its condition cannot be said to fall under either of the headings of stable or unstable equilibrium, as previously defined. Such a body as the ball is said to be in a position of "neutral" equilibrium. Its behaviour when displaced may be explained thus: Its centre of gravity coincides with its geometrical centre. The height of its centre of gravity above its base of support is therefore equal to its radius. When displaced, the height of its centre of gravity is neither increased nor diminished, and the centre is still vertically over the new point of support. Thus there is no moment of its weight about the point of support tending either to displace it further or to bring it back to its former position.

\section{Exercise for Student.}

What is the state of equilibrium of a cone made of homogeneous material when resting $(a)$ on its point; $(b)$ on its base; $(c)$ on its curved surface?

71. Other interesting points concerning the centre of gravity may be investigated by performing

EXPERIMENT 38. Using the millboard figures of which the centres of gravity have already been found 
in Experiment 36, push a pin through each of the points of intersection of the lines drawn on each figure. By moving the pin gently about, make each of the holes just large enough for the figures to move freely around the pin. Pin each figure in turn to the vertical board previously used. Note the position first taken up by each figure. Turn each into a fresh position by making it revolve around the pin. Note what happens. If the centre of gravity has been determined with sufficient care, and the hole has been made exactly at the right place, it will be found that the figure remains steady in the new position. That is to say, bodies supported at their centres of gravity are in neutral equilibrium.

Make another hole with the pin in each figure, not at the centre of gravity, and pin each in turn to the board as before. Note the position taken up. Cause each figure to revolve around the pin for a short distance, and then release it. Each will be seen to swing backwards and forwards after release and will finally come to rest in the same position as that first taken up. Note where the centre of gravity is situated with regard to the pinhole in each case when the figure comes to rest. In every case the centre of gravity will be seen to be vertically beneath the hole. From this observation we learn that bodies supported at one point, not situated at their centres of gravity, are in a position of stable equilibrium when the centre of gravity is immediately under the point of support.

Leaving the figures pinned to the board as just described, carefully adjust the position of each in turn so that the centre of gravity is vertically over the point of support. When very carefully done, it may be shown that the figure will remain in this position as long as no disturbing influence comes into action. The 
slightest disturbance, however, is enough to cause the figure to revolve rapidly around the pin, and to continue swinging till it comes to rest in the same position as formerly, i.e. with the centre of gravity vertically underneath the pinhole. Thus a body supported at one point vertically below its centre of gravity is in a condition of unstable equilibrium.

72. Familiar examples of bodies in various conditions of equilibrium may be noted, e.g. a flower-vase with a long stem and a small foot will remain balanced when filled with flowers. A slight jar, however, is generally sufficient to upset it. The centre of gravity of the vase and flowers is readily disturbed to an extent which suffices to cause the foot of the perpendicular from their joint centre of gravity to fall without the base of the vase, with the result mentioned above. The student will find it an interesting exercise to consider some more examples of bodies known to be easily knocked over, and to show that a similar explanation holds good in each case.

\section{QUESTIONS}

1. Why does a lead-pencil roll down the sloping lid of a desk ?

2. Why does a man balancing a walking-stick upon the end of his forefinger keep the end of his finger in almost constant motion ?

3. Which will overbalance more readily-a waggon loaded with hay, or a similar waggon loaded with ironmongery ?

4. Sketch what you consider a good shape for a saucepan that cannot be easily overturned. Give reasons for your opinion.

5. A common toy consists of a small man-like figure to the lower end of which is attached one-half of a spherical leaden bullet. The toy always comes to rest with the figure in an upright position, however it may be displaced. Why ? 
6. Explain why it is easier to sit on a horizontal bar than to stand on it.

7. For what reason are the legs of a three-legged stool spread outwards? (Note that the base of the stool, with respect to its stability, is the figure formed by passing a piece of string around the legs at the ground-level.)

8. The centre of gravity of the moving parts of a chemical balance is always situated at a point near and vertically below the centre knife-edge. Why ? How would the balance be affected if its centre of gravity were $(a)$ exactly at the middle point of the centre knife-edge; $(b)$ at a point immediately over this middle point; (c) at one side of the middle point?

\section{CHAPTER VIII}

\section{WORK, POWER, AND ENERGY.} THE NATURE OF HEAT

73. In Chapter III a force was defined as that which produces or tends to produce motion in a body. If the force actually does produce motion, and the point of application of the force moves in the direction of its line of action, the force is said to do work on the body. We measure the quantity of work done by finding the product of the force and the distance through which its point of application moves as described above. Thus a man pushing a wheelbarrow along a garden path is doing work on the wheelbarrow. The force exerted in this case is the muscular force applied by the man in a horizontal direction, its points of application lie at the ends of the handles of the barrow, and the distance through which the point of application moves in 
the direction of the line of action of the force is the distance the barrow travels. If this distance be 10 yards, a certain amount of work is done by the man's muscles on the barrow, while wheeling the barrow over twice that distance involves doing twice as much work. Again, suppose that a weight of $14 \mathrm{lb}$. be raised from the floor to the top of a table. A definite amount of work must be done in order to effect this. Raising a weight of $28 \mathrm{lb}$. through the same height will cause twice as much work to be performed. Further, if the $14 \mathrm{lb}$. weight be raised from the floor to a shelf at a height twice that of the table, twice as much work must be done as was required to raise it to the table-top.

74. Since work is a measurable quantity, units have been chosen in which to express the values of different quantities of work. The common British unit is "the work performed in raising a weight of $1 \mathrm{lb}$. against the resistance due to gravity through a vertical distance of 1 foot". This quantity of work is called a "footpound". In lifting a $14 \mathrm{lb}$. weight from the floor to the top of a table 3 feet high, the amount of work done is $(14 \times 3)=42$ foot-pounds. A similar unit derived from the Metric System is the kilogram-metre, i.e. the work done in raising a weight of 1 kilogram through a vertical height of 1 metre.

We have defined the work done on the body by the force above, but it does not always happen that the point of application of the force moves in the direction of the line of action of the force. Thus a man may pull at the end of a rope, attached to a moving motorcar, in the opposite direction to that in which the car is moving, but may find that this does not stop the car, and in this case the man is dragged along by the car. The point of application of the man's muscular 
force is the end of the rope, and the work done by the body-i.e. the moving car-against the force exerted by the man is measured by the product of the force into the distance through which the end of the rope travels. It will be seen that the force brought into play by the man tends to produce motion in the car, but this tendency is overcome by the resistance afforded by the force which propels the car in the opposite direction.

75. Another point deserves attention. If a $14 \mathrm{lb}$. weight be allowed to fall from the top of a table $2 \mathrm{ft} .6 \mathrm{in}$. high to the floor, the work done by the force of gravity on the weight is $\left(14 \times 2 \frac{1}{2}\right)=35$ foot-pounds. The weight may, however, be removed from the top of the table to the floor by another route. For instance, it may be caused to slide down a smooth board sloping from the table-top to the floor. The actual distance travelled over by the weight is then greater, but the distance moved over by the point of application of the force of gravity-i.e. the centre of gravity of the $14 \mathrm{lb}$. weight-in the direction of the line of action of the acting force is the same as before, viz. $2 \frac{1}{2} \mathrm{ft}$. Thus the work done by the force of gravity is the same, and is independent of the actual path taken by the weight in moving from the table-top to the floor.

76. A new idea is introduced when we consider the time taken to perform a given quantity of work. Thus the $14 \mathrm{lb}$. weight we have just been dealing with may be raised 3 feet in the air in 1 second or in 3 minutes. The work done in either case is 42 foot-pounds, but the rates of doing it are very different. Taking the second as the unit of time, the rate of working in the former case is 42 foot-pounds per second, while in the latter it is 42 foot-pounds per 3 minutes, or 
$\frac{42}{180}$ foot-pounds per second. Rate of working is termed "Power", and, as indicated above, is measured by dividing the work done by the time taken to do it. The unit of power in use in Engineering is the "HorsePower", and an engine or other body capable of doing work is said to be of 1 Horse-Power when it can perform work at the rate of $550 \mathrm{ft}$.lbs. per second. The corresponding unit in the Metric System, the kilowatt, is about $\frac{4}{3}$ H.P.

\section{Exercise for Student.}

A ton of coals is raised from the hold of a ship to the wharfside in 25 seconds. If the bottom of the hold is $18 \mathrm{ft}$. below the side of the wharf, how many horse-power is required to raise the coal ?

77. A man sitting still in a chair is doing no external work, yet we know that he is capable of doing it under suitable conditions. An engine with steam up is in similar case. Both have a capacity for working, the man by virtue of his muscular development, the engine through the expansive force of the steam produced in its boiler. The name given to the capacity for doing work is "Energy", and the man and the engine are said to possess a store of energy. It is only by the expenditure of some part or all of that store that either can perform work.

It is usual to distinguish energy as being of two kinds. Thus our $14 \mathrm{lb}$. weight, when on top of the table, possesses energy by virtue of its position. During its fall to the floor, it may be caused to do work by attaching a cord to it, the other end of which is connected with clockwork whereby a fan may be rotated, or other form of work done. The work done by gravity on the weight overcomes the resistance of the moving parts of the mechanism, and work is thus 
performed. The energy possessed by the weight because of its position is termed "Potential Energy". As long as the weight remains on the table, no work may be done by it, but it can do a certain amount of work during its fall.

Suppose the weight to be on the table as before. A nail may be placed in such a position that the weight will strike the head of the nail if it be allowed to fall from the table. On letting the weight fall, it will drive the nail into the floor for a distance which will depend on the nature of the floor and the sharpness of the point of the nail. That is to say, the force applied by the moving weight will overcome the resistance offered by the material to the passage of the nail, and the point of application of this forcethe head of the nail-will move a certain distance in the direction of the line of action of the force, i.e. vertically downwards. Thus work will be done. The moving weight, at the instant of hitting the head of the nail, evidently possesses energy by virtue of its motion. Such energy is termed "Kinetic Energy".

78. A few other examples of bodies possessing potential energy are :

(a) A bent bow.

(b) A freshly wound watch-spring.

(c) The weight of a Dutch clock while the clock is going.

(d) The muscles of a runner about to start in a race.

(e) The water in a mountain lake.

(f) The coal stored in the bunkers of a ship.

Exercise for Student.

Try to account for the possession of potential energy by these bodies by reasoning from what has been already stated. 
All moving bodies possess kinetic energy to a greater or less extent, but a few examples of such bodies as are capable of doing what is usually termed "work" (by which we understand in ordinary affairs work useful to man) are subjoined :

(a) The water of a river.

(b) A stone just before it leaves a sling.

(c) A smith's hammer as it nears the anvil.

(d) The hot air arising from a gas-flame.

(e) A circular saw, when in use.

79. A body may possess both kinds of energy at one and the same time. The water at the top of a waterfall is moving at a certain speed and hence possesses kinetic energy. By virtue of its elevated position at the top of the fall, it is capable of doing work during its fall to the bottom of the fall, i.e. it has a store of potential energy in addition to its kinetic energy. At the bottom of the fall, the water has lost the potential energy due to its position, but is moving much faster than it was before falling. That is to say, it has gained kinetic energy during its fall, and we find that, if measurements of both the loss of potential energy and the gain of kinetic energy be made, they are equal in magnitude. The potential energy is not lost, but merely transformed into kinetic energy. This relation between the loss of one kind of energy and the simultaneous production of an equal quantity of another form of energy is of universal application. If we trace with great care a certain definite quantity of energy of any kind through a series of manifestations, during which it suffers any number of changes, we are able to show that, although none of the energy may be existing in its original form, exactly equivalent 
amounts of other forms do exist as a result of the transformations undergone by the original supply. The production of sound, light, heat, and electricity are all manifestations of the change of the original energy into these widely varying forms.

80. The change of kinetic energy into heat is well illustrated by the familiar schoolboy's experiment of rubbing a button vigorously on a wooden seat. The button may be easily rendered sufficiently hot by this operation to cause considerable shock to anyone

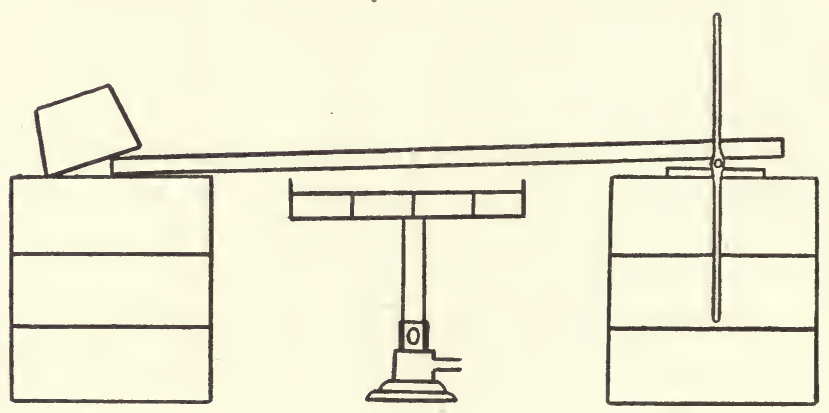

Fig. 34 .

receiving it unwarily from the experimenter. Skilful blacksmiths are said to be able to make a horseshoe red-hot by striking it repeatedly with a sledge-hammer. Another illustration of this change of kinetic energy into heat is afforded by the method of obtaining fire still practised by some uncivilised. tribes. A piece of hard wood, pointed at the end, is rotated briskly between the hands, with the pointed end pressed firmly against the surface of a small piece of wood of a softer character. By skilful manipulation the operator soon makes the softer wood begin to smoulder and glow, 
and then, by carefully fanning the glowing portion, flame is at length obtained.

81. It has been indicated in the preceding paragraphs that heat is a form of energy, or, in other words, that a hot body is capable of doing work, on account of its hotness. After the description and consideration of some simple experiments dealing with hot bodies, the nature of heat will receive further explanation.

Experiment 39. Arrange a poker, or other long bar of metal, upon sets of wooden blocks as shewn in Fig. 34. One end is kept in a fixed position by means of a $14 \mathrm{lb}$. weight, while the other rests upon a knitting-needle, which is lying on the surface of a sheet of glass placed on top of one of the wooden blocks. One end of the needle is thrust through the middle of a long straw so that the straw is at right angles to the needle. The hole in the straw must not be large enough to allow it to slip around the needle. Arrange the needle and straw in such a way that the straw is in a vertical position at the beginning of the experiment. Heat the middle portion of the poker by means of two or three good Bunsen burners. (If obtainable, a specially constructed burner, known as a Ramsay burner, which gives a Bunsen flame of a long flat shape, may be substituted for the ordinary Bunsen burners, as shown in the figure.) Soon after the heating has started, the straw will be seen to travel round in such a fashion that its top end moves forward in the direction of the end of the poker which rests on the needle. This movement of the straw will continue for some time, but at length it will reach a position in which it will remain practically steady as long as the heating with the original burners is maintained. Remove the burners and allow the poker to cool down. 
The straw will slowly revolve in the opposite direction to that previously described, and will at length come to rest in an approximately vertical position, with the same end at the top as was the case when the experiment was started.

Since the straw cannot slip round the needle, its motion must be caused by the rotation of the needle itself. This rotation is caused by the elongation of the poker under the influence of the heat supplied by the burners. The amount of lengthening is small and would be overlooked if it were not multiplied in some way, as it is in this experiment by the straw-needle combination.

If a bar of equal length to that used above, but made of another metal, such as brass, be substituted for the iron bar, similar observations will be made. If careful note be taken, however, of the number of revolutions of the straw in each case, these numbers will prove to be quite different for different metals. This indicates that different solids expand to different extents when heated similarly. For instance, the brass rod will cause the straw to make about $1 \frac{1}{2}$ times as many revolutions as a rod made of iron.

82. That the effect of heating a solid body is not merely to increase its length, but that it is also increased in bulk by being heated, may be shown by the following experiment :

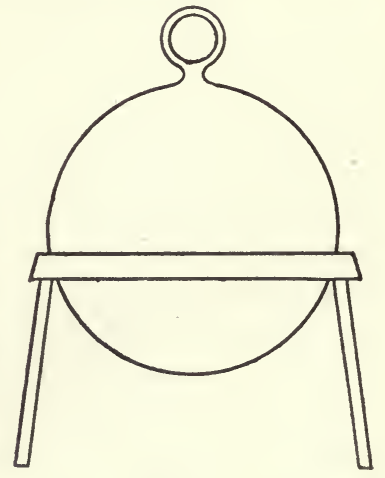

Fig. 35 .

Experiment 40. The apparatus required is that generally known as Gravesande's Ball and Ring. It 
consists of a metal ball, which just passes through a circular hole in a flat metal plate mounted upon a stand. After testing the ball as to its ability to pass through the hole when cold, heat it in a Bunsen flame for about a minute. Again try to pass it through the hole while still hot. It will no longer go through, showing that its volume has been increased, with consequent extension of its circumference.

83. To show that liquids occupy a larger volume when heated, perform

Experiment 41. Completely fill each of two similar flasks, one with water, and the other with another liquid, say alcohol. Insert into the mouth of each flask a rubber stopper, fitted with a long straight piece of glass tubing passing through a hole in the middle of the stopper. As the stoppers are pushed in, the liquids should rise in the glass tubes for a short distance. Should this not be the case, the fitting of the stopper is not good enough, or the glass tube does not properly fill the hole in the stopper. Remedy this and arrange the heights of the liquids so that the upper surface is an inch above the top of the stopper. To see the movement of the liquids better, they may be coloured with a little indigo or other colouring-matter, and the glass tubes may be pushed

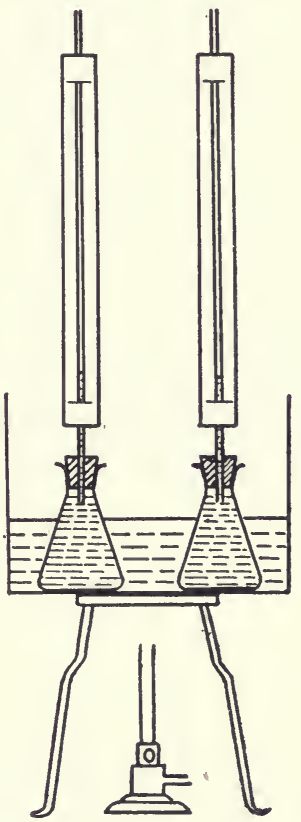

Fig. 36. 
through slits made near the ends of long strips of ruled paper, as indicated in Fig. 36.

Place the two flasks, thus prepared, side by side in the same vessel of water. Mark the positions of the liquid surfaces on the strips of paper. Gently heat the containing vessel and watch the behaviour of the liquid levels, marking the height to which each level attains when the alcohol, which will be noted as rising the faster, has nearly reached the top of the paper strip. The marking may be done most satisfactorily if the burner be withdrawn a short time before the alcohol rises to this height, and the water in the outer vessel be well stirred. Allow the whole apparatus to cool down, when it will be found that, after some time, the liquids regain their former levels (approximately).

In accordance with our previous observations with regard to the behaviour of solids when heated, the glass flasks will increase in size when heated. The liquids, however, increase to a much greater extent, and hence we are enabled to distinguish clearly the fact of their increase in bulk, in spite of the augmented capacity of the flasks.

\section{Exercise for Student.}

If the movement of the water-level in the above experiment be watched with great care, it is seen to fall slightly before the rise already mentioned. Why ?

84. Another easy experiment will be sufficient to supply us with information concerning the effect of heat upon the

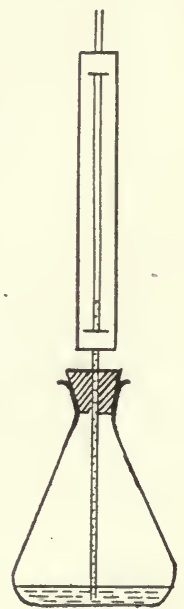

Fig. 37. volume of a gas. 
Experiment 42. Pour into a small flask enough coloured water to cover the bottom of the flask to a depth of half an inch. Fit the flask with a rubber stopper through which passes a long glass tube as shown in Fig. 37. The end of the tube should dip into the liquid at the bottom of the flask. In this way the air contained in the flask is cut off from the outside air. Arrange a paper strip behind the tube as described in the last experiment. Gently warm the flask and its contents by placing the hand around the upper part of the vessel. The coloured liquid, will be seen to rise rapidly in the tube. This rapid movement indicates that the air inside the flask has been largely increased in bulk by the heat supplied by the warm hand. The flask and the water have, of course, also gained slightly in volume, but our previous work has been sufficient to lead us to the conclusion that these slight alterations, produced by the contact of the hand, are not likely to account for the considerable expansion that evidently occurs.

If the air be displaced from the interior of the flask by passing in coal-gas from the bench-supply for about a minute, and the experiment be then repeated, it will be found that the coal-gas also shows great gain in bulk when heated in similar fashion.

Summarising the observations made in the course of the above experiments, we have

(a) All bodies, whether solid, liquid, or gaseoủs, expand when heated.

(One rather important exception to this rule will be dealt with fully in Chapter xvIr.)

(b) The expansion produced is most marked in the case of gases, less with liquids, and least with solids. 
(c) Solid bodies made of different substances expand to different extents when similarly heated.

(d) Liquids also vary with respect to the increase shown when heated under similar conditions.

We have not yet made any experiment with gases from which we can strictly compare their behaviour when similarly heated. For the present we may state that equal volumes of all gases show increases of volume which are practically identical when their temperatures are raised by equal amounts.

Condensing our observations into a single statement, this is as follows :

One effect of heat upon bodies is to increase their volumes.

85. Some further experiments will afford us information with regard to another notable change in a body which may be brought about under the influence of heat.

Experiment 43. Fill a small porcelain dish with lumps of ice. Heat the dish over a small Bunsen flame and note the effect produced on the ice. Continue the heating till the water boils. Boil it steadily, so that no liquid can spirt out of the dish. When no further change can be noted, remove the burner and examine the contents of the dish.

If clean ice be used, some of our observations will be that the solid ice becomes liquid water, that the liquid, after further heating, disappears, and that no appreciable quantity of matter is left in the dish. Since we are unable to see the substance into which the ice is finally changed, we might incorrectly infer that the matter contained in the ice no longer exists. This is not the case, however, and the matter of the ice is still in existence, after the completion of the experiment, 
as an invisible vapour diffused through the air of the room in which the experiment is carried out. This vapour we know as steam, and, by holding a clean cold object over the dish while the water is boiling, some of the steam may be recovered in the form of water, which forms small drops on the outside of the cold object.

By similar treatment of other solids, it may readily be shown that, in many cases, the application of heat causes them to assume the liquid form, which may be again transformed into a solid by allowing the liquid to cool. Again, most liquids, when heated, may be caused to assume the gaseous state, and, on cooling, become liquid once more.

As the general result of the above considerations, we may say that

Another effect of heat upon bodies is to bring about a change in the state of matter in which they exist.

86. Expertment 44. Into a small test-tube put about 1 gram of the red powder called red oxide of mercury. Heat the end of the tube containing the oxide in a small Bunsen flame. The first change noted will be that the powder assumes a darker hue, becoming brown and finally almost black. Stop the heating at this stage and allow the tube and its contents to cool. The oxide will slowly change in colour once more, the changes taking place in the reverse order to that first noted, until its appearance is not distinguishable from that of some of the same powder that has not been heated. Heat up the oxide again, and continue heating after the colour has reached its deepest shade. Ignite a thin splinter of wood in the flame, blow out the flame of the wood, and insert the still glowing end of the splinter into the upper end of the tube. It will be 
found to burst into flame, and to burn with greater brilliancy than was the case when it was burning in air. The partial extinction and re-ignition of the splint may be effected several times - in fact, as long as there remains any powder at the end of the strongly heated tube. When all the powder has disappeared, discontinue the heating, and examine the walls of the tube just above the portion which has been heated. A thin silvery metallic deposit is to be seen, which, on being rubbed with the end of the splint, collects into small globules easily recognised as quicksilver or mercury.
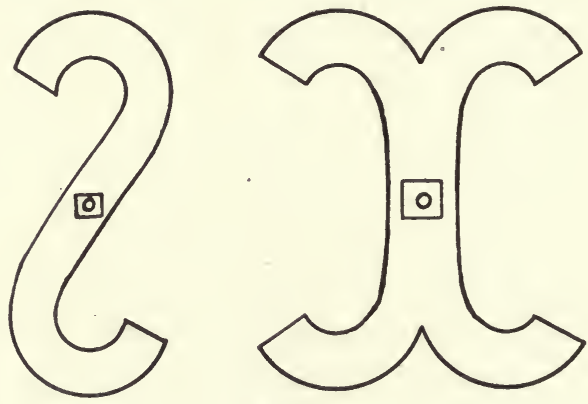

Fig. 38 .

In this case, two effects of the action of heat have been shown. The first was the change of colour, the second the disappearance of the original substance and the simultaneous appearance of two new bodies, the gas which re-ignited the glowing splint and the mercury left in the tube. The former change resembled those previously observed regarding the action of heat upon bodies, in that the body was not completely altered by the change, but could be readily recognised as the same substance after the withdrawal of the disturbing effect of the heat. Such changes are distinguished as 
"physical" changes. In the latter change in the mercury oxide, however, the mercury oxide ceases to exist as such, and by no simple means, such as the withdrawal of the source of heat, may it be again produced from the new bodies obtained from it. Changes of this character are called "chemical" changes. Hence a further general statement may be made as follows:

A third effect of heat upon bodies is to cause the formation from them of entirely distinct bodies.

87. It may be readily shown that work may be performed during each kind of change mentioned above, or, in other words, that heat is a form of energy. Thus, when a body is caused to expand by the application of heat, very great force may be produced, and similarly, when the body cools, equally great forces may be brought into play. An example of this is seen in the method sometimes used for strengthening old brickwork. On old walls one may see straps of iron of the shapes shown in Fig. 38. In the middle of each strap is a nut which travels upon a screw thread worked on the end of a long iron rod passing through the wall, and provided at the other end with a similar strap and bolt which are affixed to the opposite wall of the house. The object of this arrangement is to prevent the walls of the house from falling outwards, and the manner in which this is effected is as follows: The rod is placed in position with the straps and nuts loosely fitted upon its ends. It is then strongly heated with a consequent increase in length. While still very hot, the nuts are screwed home as tightly as possible. The source of heat is then withdrawn and the rod allowed to cool. In cooling it contracts and the force of contraction is sufficient to counteract the bulging of the walls, thus rendering the house a safer habitation. 
The change of water into steam is attended by an enormous increase of bulk, the steam produced from a given volume of water occupying more than 1600 times the volume of the water from which it was formed. If the water be boiled in a confined space, such as the boiler of a steam-engine, great increase of pressure results as a consequence of the production of steam, - and it is this pressure which sets in motion the movable parts of the engine and causes it to perform work.

That chemical change, when effected by heat, is also capable of doing work may be easily grasped when one considers the result of the explosion of a blasting cartridge, loaded with dynamite and packed tightly into a small hole drilled into the rocks in a quarry, when the burning end of the fuse attached to it transmits heat in sufficient quantity to cause the constituents of the dynamite to change into new bodies. Most of these are gases and occupy many thousand times the volume of the original explosive. Their sudden production, assisted by their very considerable expansion under the influence of the large quantity of heat simultaneously liberated during the chemical change, is enough to split open even the hardest rocks.

In conclusion, we will emphasise the general inference obtainable by consideration of the matters dealt with in this chapter, viz.

Heat is a form of energy.

\section{QUESTIONS}

1. A cylindrical leaden clock-weight, $6^{\prime \prime}$ long, weighs $12 \mathrm{lb}$. When the clock is fully wound up, the bottom of the weight is $8 \mathrm{ft}$. 4 in. from the floor. Express the potential energy of the weight in this position in foot-pounds. 
2. Classify the following bodies as possessing $(a)$ kinetic energy ; (b) potential energy; (c) both kinetic and potential energy:

(1) A sack of flour on a man's back.

(2) The arms of a windmill in motion.

(3) A barrel of gunpowder on the top of a wall.

(4) A kite suspended in the air at the end of a string.

(5) A kettle full of boiling water resting on the top of a kitchen range.

(6) A rifle-bullet just leaving the muzzle of the gun.

3. Why do the bars of a fire-grate usually fit loosely in their sockets ?

4. Why does the thread of mercury in a thermometer move up and down ?

5. Straight lengths of railway line, if laid in the winter with the end of each length pressed closely against those of its neighbours, become bent during the hot weather of summer. What is the reason of this ?

6. What kind of energy is possessed by the fire in a range ? Give the reason for your opinion as fully and clearly as you can.

\section{CHAPTER IX}

\section{TEMPERATURE. THERMOMETERS}

88. In everyday life we speak of one body being hotter or colder than another, and our opinion is in general based upon information afforded by our bodily sensations. No difficulty is experienced in judging by this means whether a red-hot lump of coal is hotter than a kettle-lid, or a piece of ice colder than a cup of tea. It is far from easy, however, to determine by touch whether one glass of cold water is exactly as cold as another. Further, it is possible to obtain contradictory evidence on this point with reference to whether a body is hot or cold by asking the opinions of different people, and the same person will not always 
give the same opinion with regard to the same body in the same condition with respect to its hotness. A frequently quoted experiment may be performed in illustration of this.

Experiment 45. Procure three bowls, such as.are in common use for kitchen purposes. In one pour some water as hot as the hand is capable of bearing, into the second some cold water from the tap, and into the third a mixture of equal parts of the hot and cold water. Put the right hand into the hot water, and the left into the cold. Allow each hand to remain in the water until no special feeling of heat or cold is to be noticed in either case. Remove the hands and plunge both into the third bowl. The right hand will provide the information that the water in this bowl is cool, while the left gives the impression that the same water is distinctly warm.

From what has been shown in this experiment it is clear that one's bodily sensations are not trustworthy guides in the matter of measuring the degree of hotness of a body. Many instruments have been devised by which this measurement may be effected with greater accuracy, the one in most general use being the mercury thermometer. (Gr.thermos, heat.) In this instrument and in the alcohol thermometer advantage is taken of our earlier observation to the effect that liquids in general expand under the influence of heat to a greater extent than solids.

89. A thermometer consists essentially of a long glass tube of fine bore, upon one end of which a bulb has been blown, and the other end of which is sealed. At ordinary temperatures the bulb and part of the stem are filled with mercury, the remainder of the stem being empty, save for a minute quantity of the vapour 
of mercury. When the thermometer is placed in contact with a body hotter than itself, heat is given up to the thermometer by the hot body, and the mercury expands and hence rises in the tube. On the other hand, when brought into contact with a body colder than itself, the thermometer gives up heat to the body, with the result that the volume of the'mercury becomes less and the thread of liquid in the tube sinks.

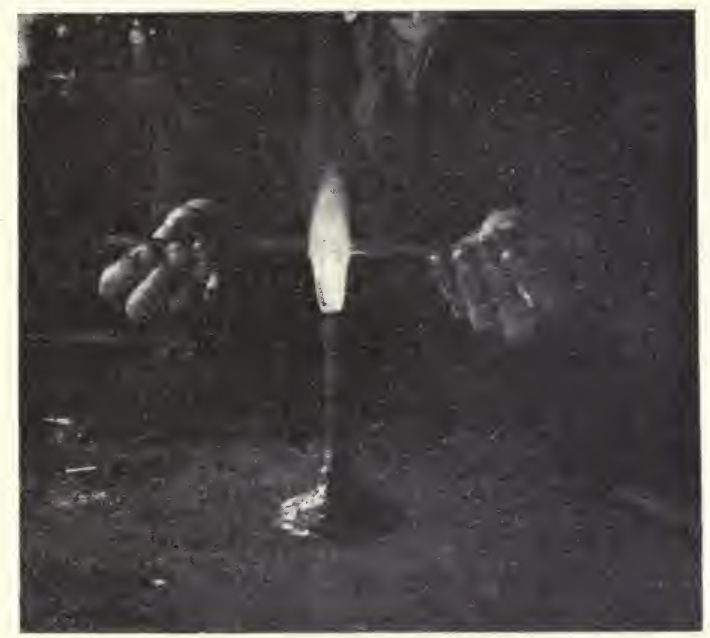

Fig. 39.

The method of construction of a thermometer is of interest since it introduces some applications of principles previously investigated. The making of a mercury thermometer is too delicate an operation for general practice, but the method is similar to that described in the next experiment dealing with the construction of an alcohol thermometer.

Experiment 46. A piece of thick-walled glass tubing of fine bore-usually called "capillary tubing" 
-about $20^{\prime \prime}$ long must first be procured. Heat the tubing at a point about $2^{\prime \prime}$ from one end in a Bunsen flame, the tubing being held as shown in Fig. 39. During the heating the tube should be rotated backwards and forwards between the fingers so that it. is evenly heated throughout. When the heated portion of the glass has become thoroughly soft, remove it from the flame, and gently pull each end of the tube in a horizontal direction till the softened part has been stretched into a small thin tube about 6 to 8 inches long. Allow the tube to become quite cold. Cut the tubing by making a scratch on it with a sharp file at the end of the drawn-out portion nearest the longer part of the tube, putting a thumb and forefinger on each side of the scratch, with the mark on the side of the tube away from the body, and snapping the glass as one breaks a small twig. The longer piece of tubing will then present the appearance shown in Fig. 40. A bulb is next blown at the pointed end of this tube by carefully carrying out the following instructions. Heat the pointed end by holding it just above the blue inner cone of a strong Bunsen flame, continually rotating the tube as before. The end of the tube will become closed by the glass, when sufficiently softened, gradually forming a small semi-liquid drop at the end of the heated part. At this stage remove the tube from the flame, and gently blow with the mouth at the open end of the tube. As the tubing cools, the strength of the blast Fig. 40. may be increased. This should result in the formation of a small swelling at the closed end. Repeat the heating till the tube is again thoroughly 
softened, blow as before, and the small bulb should become somewhat larger. These processes should be repeated till the bulb obtained is about $\frac{1}{2}$ in diameter, and its wall is of even thickness. The latter point may be tested by gently knocking various parts of the bulb upon the top of the bench, when the bulb has cooled down. The nature of the sound produced will vary if different parts of the wall are of different thicknesses. The production of a good bulb requires some care and it may be found that two or three attempts will have to be made before a really satisfactory. one is obtained. Special attention must be paid to the direction to continue the heating till the glass is thoroughly softened, as it is useless to attempt to blow a bulb from insufficiently heated glass.

Next draw out the other end of the tube at a point about $2^{\prime \prime}$ from the end in the manner already described. Cut off the thin tube close to the shorter piece of tubing. Bend the thin tube near its junction with the thick-walled part by heating it at this point carefully, removing it as soon as it shows signs of softening, and gently bending it into the shape indicated in Fig. 4l. Arrange the apparatus as in the figure. The bulb is immersed in a beaker of water, while the open end of the tube dips into a small beaker containing freshly distilled alcohol to which sufficient colouring-matter, such as

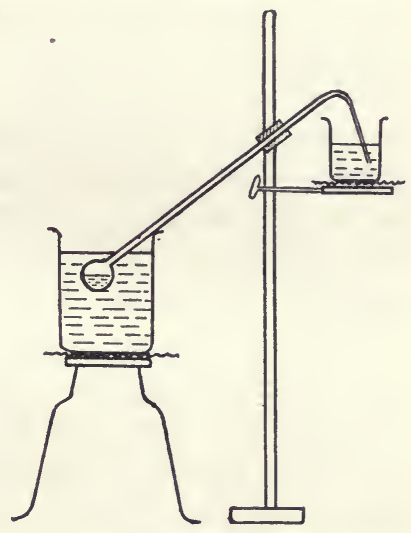

Fig. 41. litmus, has been added to render it more clearly visible 
when in the tube. Heat the water in the larger beaker to boiling. Bubbles of air will escape through the coloured liquid as the water becomes hot. After the water has boiled for half a minute, remove the beaker and let the bulb cool down, leaving the open end of the tube under the surface of the alcohol. As the tube cools, some of the coloured liquid will be seen to travel along the tube into the bulb. When this action appears to have ceased, replace the beaker containing the warm water, and again heat it to boiling-point. It will be found that the alcohol in the bulb rapidly boils away, leaving the bulb and tube apparently empty. Again remove the hot water, and allow the apparatus to cool. As before, the alcohol will be driven into the bulb and this time the bulb will be found to become almost entirely filled with the coloured liquid. By repeating the heating and cooling, it is possible to expel all but the smallest trace of air from the bulb and tube. Let the bulb and tube cool down at this stage till it no longer appears warm to the hand, leaving the open end of the tube under the alcohol as before. We will suppose that it is desired to show on the finished thermometer the ordinary temperatures experienced in this country. To this end, surround the bulb with water at a temperature of about $40^{\circ}$, as registered by a Centigrade thermometer: Stir the water well, and after leaving the bulb in the warm water for a minute, seal off the upper end of the thermometer by applying a small blowpipe Fig. 42. flame to the end just beyond the tapering part. (Instead of a blowpipe flame, the flame obtained by unscrewing the barrel of a Bunsen burner, and lighting the gas at 
the fine jet, may be used. A flame about $1^{\prime \prime}$ high will be found most convenient for the purpose.) As soon as a satisfactory seal has been obtained remove the warm water and allow the thermometer to cool. By cautiously heating the glass at the sealed end in a small flame, with constant rotation, a small ball of glass may be formed, which will give a better finish to the thermometer. It will then have an appearance similar to that shown in Fig. 42.

To graduate our instrument, it should be placed with its bulb in a quantity of finely pounded ice, as illustrated in Fig. 43. Allow it to remain in the ice for some minutes, noting at intervals of one minute the position of the upper surface of the liquid. When no further fall has occurred between two such observations, mark the level of the alcohol by making a light scratch on the glass with a sharp file. This point will mark for future work with the thermometer the melting-point of ice, and may be called the zero of our scale. To obtain some further points on the scale, place the thermometter side by side with a Centigrade thermometer in the same vessel of water, and, by adding small pieces of ice (if necessary) adjust the temperature of the water to $10^{\circ} \mathrm{C}$. The water must be stirred continuously, so as to be of the

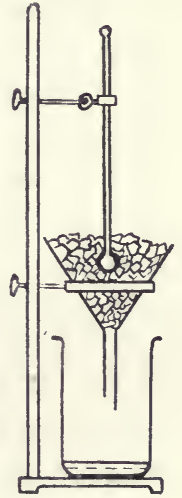

Fig. 43. same temperature throughout. When a steady temperature of $10^{\circ}$ has been attained, mark the level of the alcohol as before. By gently heating the water to $20^{\circ} \mathrm{C}$. and $30^{\circ} \mathrm{C}$. in turn, further points on our scale may be marked. The intermediate divisions may be marked off by dividing each part of the 
tube between the marks already made into 10 equal parts.

The actual method of making a mercury thermometer is very similar to that described above, but precautions are taken with regard to the evenness of the bore of the tube, the dimensions of the bulb in relation to the bore, and the expulsion of the last traces of air in the filling, which are not necessary in the performance of an experiment such as ours, in which the illustration of the processes used rather than the production of a standard article, is the chief point to be considered.

90. We obtained one of the points on our scale by finding the level at which the liquid stood in the bore of the thermometer when the bulb of the latter was completely immersed in melting ice. The corresponding point on the scale of a mercury thermometer is obtained in a similar manner.

Expertment 47. Check the accuracy of the freezingpoint as marked on the laboratory thermometers by repeating that portion of the preceding experiment dealing with the determination of this point, replacing the alcohol thermometer by each of those to be tested in turn. Allow each to remain in the ice till the mercurylevel remains steady for two minutes and note the actual reading on the scale when this occurs. According to the method of graduation adopted, the readings should be $0^{\circ}$ for a Centigrade, and $32^{\circ}$ for a Fahrenheit thermometer. Unless the thermometers tested are expensive ones, it will probably be noted that the actual readings are slightly greater than they should be. This is due to the fact that the bulb continues to contract for a very long time after the making of the thermometer, and hence the volume of mercury it can 
contain is lessened, with the result that some is driven into the capillary tube.

91. A second fixed point is obtained on a mercury thermometer by noting the level to which the liquid rises when the instrument is immersed in the steam from boiling water. This point could not be marked on our experimental thermometer, since alcohol boils at a lower temperature than water. To check the boiling-points of the laboratory thermometers, we will do

Experiment 48. Arrange the apparatus shown in Fig. 44. The best check is obtained when the boilingpoint of the instrument- $100^{\circ}$ C. or $212^{\circ}$ F.-just appears above the cork. Heat the water until it boils freely, and allow boiling to continue till the top of the mercury column in the thermometer has remained steady for two minutes. The readings should be very close to those quoted above, but slight variations may be noted, which may possibly be due to the decrease in the capacity of the bulb already mentioned, or to the pressure of the laboratory being above or below the standard pressure, $760 \mathrm{~mm}$.

If the behaviour of the liquid be carefully watched during this experiment, it will be noted that small bubbles are formed at an early stage of the heating, chiefly upon the bottom of the flask. These gradually increase in size and at

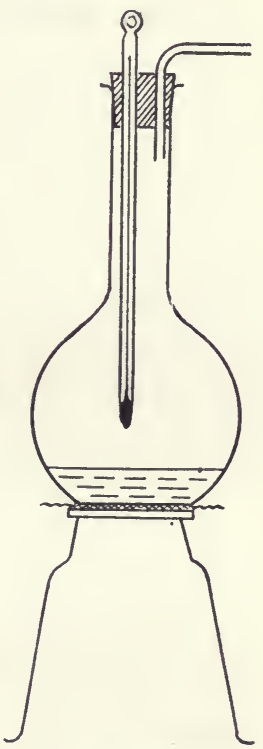

Fig. 44 . 
length detach themselves from the interior of the vessel and rise to the top of the liquid. The bubbling thus produced must not be mistaken for boiling. The small bubbles are not bubbles of steam but of air, which was dissolved in the water and is driven out of solution by heating the liquid. At a later stage somewhat larger bubbles may be seen to form at the bottom of the flask and to rise a short distance, but not to reach the top of the water. These are the first steam-bubbles, which are formed at the hottest part of the liquid but are condensed to water again on coming in contact with the cooler water during their upward passage. These bubbles of steam become larger and more numerous as the heating proceeds and rise to a greater height before collapsing. At length the bubbles reach the top of the liquid and burst there. As soon as steam is thus escaping freely at all parts of the surface of the liquid, the true "boiling-point" has been reached. In order that the steam may thus reach the surface of the water and pass freely into the space above it, the pressure of the steam must be at least equal to that of the air which fills this space, or the air will not be pushed away by the steam.

Boiling, or "ebullition", must be distinguished from "evaporation". In the latter process the formation and escape of vapour take place only at the free surface of the liquid, while

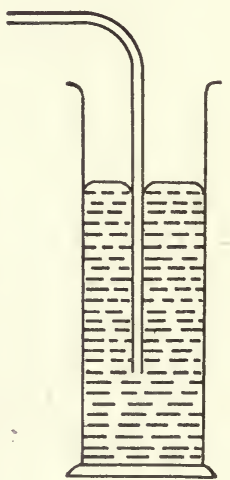

Fig. 45 . during ebullition steam is formed in all parts of the water. Ebullition occurs at a certain temperature, dependent upon the exterior pressure on the surface of 
the liquid; evaporation takes place at all temperatures though much less rapidly at lower temperatures.

92. To show how the boiling-point of a liquid is affected by variation of the pressure to which its vapour is subjected, two simple experiments may be performed.

Experiment 49. Fit up the apparatus illustrated in Fig. 44, and attach the outlet tube to the end of the long bent tube which dips under the surface of the mercury in a jar to a depth of about $20 \mathrm{~cm}$. as shown in Fig. 45. Heat the water in the flask to boiling-point, and note the reading of the thermometer when the water is boiling freely. It will be found to be some 6 to 7 degrees higher than that previously observed when the water was boiling under atmospheric pressure.

\section{Exercise for Student.}

If the atmospheric pressure at the time of the experiment be $750 \mathrm{~mm}$., and the end of the tube is at a depth of $20 \mathrm{~cm}$. below the surface of the mercury, under what pressure is the water boiling ?

EXPERIMENT 50. Obtain a well-made round-bottomed flask. Half fill it with water and heat the water till it boils. While still boiling, remove the source of heat and quickly insert a well-fitting stopper. Invert the flask upon a tripod stand as shown in the accompanying figure. When no more bubbling takes place pour cold water over the outside of the flask. This will cause vigorous boiling to take place. When no more bubbles appear, showing that boiling has again ceased, pour a further supply of cold water over the flask.

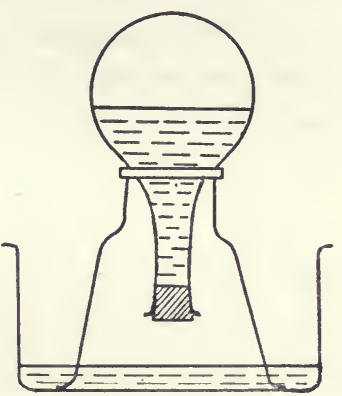

Fig. 46. 
Further boiling at once takes place, and this phenomenon may be caused to occur several times.

It will be well to consider rather closely what happens in this experiment, in order to account for the strange fact that cooling the flask makes the water start boiling again. At the instant of corking the flask the upper portion of the latter is filled almost entirely with steam, the small remaining portion being air which has not been completely expelled by the steam. When cold water is poured over the bottom of the flask, some of this steam, which is in the vaporous condition, is rapidly condensed to water, and this water only occupies an extremely small volume as compared with that of the steam from which it was made. The pressure of the remaining steam above the water is much less than that previously exerted by the whole quantity of vapour originally present. To restore in some part the original conditions, some of the water is vaporised, with the consequent appearance of bubbles of steam in the liquid. Further disturbance of equilibrium by repetition of the cooling is followed by a further outbreak of ebullition.

The important observations made in Experiments 49 and 50 may be summed up thus:

The temperature at which a liquid boils is dependent upon the pressure to which its free surface is subjected, increase of pressure raising the boiling-point, while diminution of pressure has the opposite effect.

93. The two fixed points - the melting-point of ice and the boiling-point of water-having been marked upon the scale of a mercury thermometer, the space between these two points is divided into a number of equal parts, called degrees. Two scales are in use in this country. In that found on most thermometers in domestic use, the number of degrees between freezing 
and boiling points is 180. The freezing-point is marked $32^{\circ}$ and the boiling-point $212^{\circ}$. This scale is due to Fahrenheit, and thermometers thus marked are called by his name. The graduations are usually continued above and below the fixed points to an extent which depends upon the purpose for which the thermometer is intended. In all scientific work a scale in which the freezing-point is marked $0^{\circ}$ and the boiling-point $100^{\circ}$ is used. This is the Celsius, or, as it is more commonly termed, the Centigrade, scale. A third method of graduation, due to Réaumur, is in common use on the Continent, more especially in the northern countries. In this the freezing-point is marked $0^{\circ}$ and the boiling-point $80^{\circ}$.

Temperatures below zero on any scale are expressed by counting the number of degrees from the zero mark in the opposite direction to that in which temperatures above zero are counted, such low temperatures being distinguished by prefixing the minus sign. Thus a temperature of $10^{\circ}$ below the zero of the Centigrade scale would be written $-10^{\circ} \mathrm{C}$.

Temperatures expressed according to any one of these methods may be changed into their corresponding values on either of the other scales by noting that $80^{\circ}$ Réaumur $=100^{\circ}$ Centigrade $=180^{\circ}$ Fahrenheit, and that, in changing degrees Fahrenheit into either Réaumur or Centigrade readings, $32^{\circ}$ must be first subtracted from the Fahrenheit value, while, in effecting the reverse change, $32^{\circ}$ must be added to the value obtained.

Example: Transform $85^{\circ} \mathrm{C}$. into degrees F., and $15^{\circ} \mathrm{F}$. into degrees $\mathrm{R}$.
(a)
$85^{\circ} \mathrm{C} .=\left(85 \times \frac{180}{100}\right)^{\circ} \mathrm{F} .=153^{\circ} \mathrm{F}$. 
To this must be added $32^{\circ} \mathrm{F}$, giving as a result $185^{\circ} \mathrm{F}$.

(b) Subtracting $32^{\circ} \mathrm{F}$. from $15^{\circ} \mathrm{F}$. gives $-17^{\circ} \mathrm{F}$. $-17^{\circ} \mathrm{F} .=-\left(17 \times \frac{80}{180}\right)^{\circ} \mathrm{R} .=-7 \cdot 6^{\circ} \mathrm{R}$.

94. We have already spoken of heat being transmitted from one body to another, and one's daily experience is sufficient evidence that this transfer actually takes place. We have not yet considered, however, under what circumstances heat may thus be transferred. A little thought will convince one that it takes place whatever the materials, the sizes, the densities, and the physical states of the bodies. Thus a small body will give up heat to, or receive heat from, a larger one-a liquid may receive heat from, or supply heat to, a solid-and so on. The condition which must be fulfilled in order that heat may pass from one body to another is that they must be at different temperatures, i.e. one body must be warmer than the other. We are thus led to formulate the usual definition of temperature as follows:

The temperature of a body is its condition with reference to its capacity for receiving heat from, or supplying heat to, other bodies. Those bodies from which it receives heat are said to possess a higher temperature than the body itself, those which it supplies with heat are at a lower temperature, and those which neither receive nor supply heat are said to be at the same temperature.

The interchange of heat is however regarded as a continuous process, and as going on between bodies at the same temperature as well as between those at different temperatures. In the latter case, however, the amounts of heat received and supplied by either body are equal, 
and thus neither becomes hotter than at first. It will be noticed that during the passage of heat from a hot body to a colder one, the temperature of the hot body diminishes, while that of the cold one increases. This continues till both bodies are at the same temperature. Thus, when we determine the temperature of a body by placing a thermometer in contact with it, the instrument receives heat, if the body be warm, and the final temperature recorded on the thermometer will be that at which the quantities of heat interchanged are equal. This will be evidently slightly lower than the original temperature of the body, but the error will in general be small, since most bodies treated in this way are considerably larger than the thermometer and the amount of heat lost in warming up the thermometer is comparatively little. This point will be more clearly grasped after reading the next chapter.

95. Two useful modifications of the ordinary thermometer merit special description. The clinical thermometer, of which Fig. 47 is an illustration, has a scale of short range, usually from $95^{\circ} \mathrm{F}$. to $110^{\circ} \mathrm{F}$. The degrees are further divided into fifths or tenths of a degree. The bore of the instrument is small, and, in order to enable the position of the end of the fine mercury thread to be read with ease, the shape of the section of the glass tube is such that the glass has a magnifying effect. A short Fig. 47. distance above the bulb of the instrument the bore of the tube is narrowed considerably. The purpose of this constriction is to prevent the mercury, which has passed into the part of the tube on which 
the scale is marked, from returning to the bulb immediately the thermometer is removed from the mouth or armpit of the patient. The doctor or nurse is thus able to discover the body temperature of the patient after the removal of the clinical thermometer. To prepare the instrument for further use, it is held by the end further from the bulb and gently swung with a circular motion at arm's length. The thread of mercury is thus caused to pass the constriction into the bulb.

\section{Exercise for Student.}

Determine suitable ranges of scale for clinical thermometers in which the Centigrade and Réaumur scales of temperature are used.

The observation of the lowest or highest temperatures recorded in a given position during a known interval is often of service. For use in this connection, specially fitted thermometers are used. Fig. 48 shows a portion of the stem of a thermometer designed to register mini-

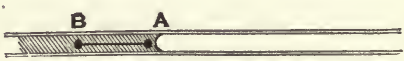

Fig. 48. mum temperatures. The liquid made use of is alcohol and a small steel index, shaped like a dumb-bell, is immersed in the alcohol. The thermometer tube is placed in a horizontal position. The extremity of the alcohol column, in moving towards the bulb as the temperature falls, carries with it the index, since the resistance offered by the latter is insufficient to cause it to break through the surface of the liquid. On the other hand, a rise in temperature does not affect the position of the index, the alcohol passing freely without moving it. Thus the end of the index further from the bulb of the instrument shows the lowest point reached by the liquid, and hence the minimum temperature which has been experienced 
at the place of observation, since the thermometer was last set. To set the index, a small magnet is passed along the tube in the direction away from the bulb, and the steel index follows it until the meniscus of the liquid is reached.

Fig. 49 is an illustration of a portion of the stem of a maximum thermometer. Mercury fills the bulb and part of the stem, and a steel index, provided with spring-like ends as shown,

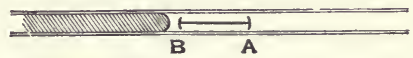

Fig. 49 . is contained in the space above the mercury. The steel is not wetted by the liquid, and when the end of the mercury column in contact with the index moves along the tube while the temperature is rising, the index is carried before it. On the retreat of the liquid during a fall of temperature, the index retains its position. The end of the index nearer the top of the mercury column thus registers the maximum temperature recorded during the period of observation. To reset the instrument, a magnet is used as indicated in the case of the minimum thermometer. The stem of this thermometer is also kept in a horizontal position.

The above thermometers are known as Rutherford's minimum and maximum thermometers. In another form, both minima and maxima of temperature are registered by the same instrument. Six's thermometer consists of a large bulb containing alcohol to which is attached a long bent tube containing mercury in one bend as shown. Two small indexes, provided with springs, are placed in the alcohol at each end of the mercury column. The remaining portion of the tube and part of the cup at the top contain alcohol. Expansion of the alcohol in the bulb and first part of 
the tube causes the further end of the mercury column to push the index in front of it till the temperature ceases to rise. The index stays in its new position by virtue of the spring. Thus the temperatures registered by the scale on the limb further from the bulb are maxima. Similarly, the other index is pushed back toward the bulb during a drop in temperature, and the point on the scale at which its lower end comes to rest is the minimum temperature experienced since the last setting of the index. The indexes are once more brought into contact with the ends of the column of mercury, after observations have been made, by means of a magnet.

96. In Chapter viII it was demonstrated that gases undergo large changes of volume as the result of rise or fall of temperature. The measurement of the change of volume consequent upon a given change of temperature may be approximately estimated by performing

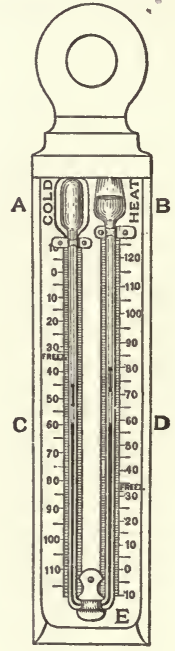

Fig. 50 .

Experiment 51. Draw out a piece of capillary tubing as described in Experiment 46. Cut off the drawn-out portion so as to obtain a tube about $20^{\prime \prime}$ long, similar to that shown in Fig. 40. Pass dry air through this tube for some minutes and then seal the pointed end, by heating it in a Bunsen or a blowpipe flame. Heat the tube thus prepared by passing a flame backwards and forwards along its length several times. While it is still warm, put the open end under the surface of some mercury in a dish and let the tube cool a little. When a thread of mercury of about half an inch in length has entered the tube as a result 
of the cooling, remove the end of the capillary from the liquid, and allow it to cool to the temperature of the room. At this stage the thread of mercury should be distant from the open end of the tube by about onethird of the length of the tube. Should the distance be less than this, heat up the tube once more for a somewhat longer time than formerly, and repeat the immersion in mercury and subsequent cooling to room temperature. Place the tube in a large vessel of water, a mercury thermometer being immersed close by, and after a minute read the temperature recorded by the mercury thermometer. Make a small scratch with a file on the capillary tube at the point reached by the lower end of the mercury thread under these conditions, the tube being held in a vertical position. Heat the vessel and water until the thermometer shows a rise of about $20^{\circ}$. Remove the source of heat, stir well and again read the thermometer and mark the tube as before. Heat up the apparatus again till a further rise of $20^{\circ}$ is shown and again mark the tube. About four such determinations of the position of the lower end of the mercury thread may be obtained. These may be checked by allowing the vessel and its contents to cool with frequent stirring of the water, carefully observing whether the end of the mercury column reaches the same position as previously, when each of the temperatures at which the original observations were made is attained during the cooling.

If we neglect any slight unevenness in the bore of the tube and any small irregularity of shape at the closed end, the lengths measured from the closed end to the various marks made. on the tube will be proportional to the volumes of the mass of air, imprisoned in the tube between the closed end and the under 
surface of the mercury, at the various temperatures noted. To obtain the amount of expansion of unit volume corresponding to a change of $1^{\circ}$ in temperature, we proceed as follows:

Suppose that the length of the air-column at $17^{\circ}$ is $13.8 \mathrm{~cm}$. and that at $39^{\circ}$ it measures $14.85 \mathrm{~cm}$. Thus we have that volume of air represented by 13.8 increasing by $(14.85-13 \cdot 8)=1.05$ when the temperature is raised $22^{\circ}$. The increase for a rise of $1^{\circ}$ is $1 \cdot 05 \div 22=\cdot 048$ (approx.). Each unit volume of air has increased by $\cdot 048 \div 13 \cdot 8=\cdot 0035$ of its original volume at $17^{\circ}$ for each degree rise of temperature.

Perform similar calculations to the above for each pair of temperatures registered in your experiment, using the original temperature as one of the pair, and find the mean value of the results. This should be of about the same magnitude as that found in the example given.

97. By making the original temperature $0^{\circ}$ by floating ice in the water till its temperature has fallen to freezing-point, we may find how much unit volume of air expands when its temperature is raised by unit amount. Very careful determinations of this quantity have been made, and its value has been shown to be 0.003665 , or, expressed as a vulgar fraction, $\frac{1}{273}$. By replacing the air by other gases, it may be shown that the same value is obtained for all of them, with slight variations only. This observation may be put in the form of a general statement:

All gases expand by 0.003665 of their volumes at $0^{\circ} \mathrm{C}$. when their temperatures are raised by $1^{\circ} \mathrm{C}$.

This statement is true only when the pressure of the 
gas does not alter during the expansion, since alteration of pressure would cause change of volume on its own account and thus might counteract or augment the effect properly due to the heating alone. Dalton in England and Charles in France independently discovered this regularity in the expansions of gases and the statement given above is variously known as Charles's or Dalton's Law.

98. Examples of the use of the law in calculations involving expansion or contraction of gases under the influence of heat may be best worked as below:

(a) How much air will escape from a room 5 metres long, 3 metres high, and 4.5 metres wide, when the average temperature of the room changes from $10^{\circ}$ to $21^{\circ}$ ?

Interior volume of room $=5 \times 3 \times 4.5=67.5 \mathrm{cu}$. $\mathrm{m}$. Each cubic metre at $10^{\circ}$ will have at $0^{\circ}$ a volume of $\frac{273}{83} \mathrm{cu}$. m. Since the increase in volume of each cu. $\mathrm{m}$. for a rise of $1^{\circ}$ is $\frac{1}{2} \frac{1}{3}$ of its volume at $0^{\circ}$, each cu. $\mathrm{m}$. at $10^{\circ}$ will gain $\frac{1}{2} \frac{1}{3} \times \frac{273}{283} \mathrm{cu}$. m. for each rise of $1^{\circ}$. The total increase in volume will therefore be

$$
67.5 \times 11 \times \frac{1}{283}=2.62 \mathrm{cu} . \mathrm{m} .,
$$

i.e. this volume of air will escape from the room.

(b) At what temperature will 100 cubic feet of coal-gas measured at $15^{\circ}$, become $101 \mathrm{cu}$. ft.?

By a similar line of argument to that used in the former example, it may be shown that each $\mathrm{cu}$. ft. at $15^{\circ}$ gains in volume by $\frac{1}{288} \mathrm{cu}$. ft. for each rise of $1^{\circ}$. Hence the rise in temperature is $(101-100) \div \frac{100}{288}$ $=2.88^{\circ}$ and the required temperature is $15+2 \cdot 88$ $=17 \cdot 88^{\circ}$.

99. Let us next consider the decrease in volume of 273 c.c. of a gas at $0^{\circ}$ when its temperature is lowered 
below $0^{\circ}$. If we assume Charles's Law, the volume at $-1^{\circ}$ will be 272 c.c., that at $-10^{\circ}$ will be 263 c.c., that at $-100^{\circ}$ will be 173 c.c., and so on. It is easy to see that continued application of this reasoning will give 0 as the volume at $-273^{\circ}$. Long before this temperature is reached, most gases become liquids, and we know of no substance, however difficult it be to liquefy, that remains in the gaseous state below $-268^{\circ}$. Liquids do not obey Charles's Law so that the decrease of the volume of a substance to nothing is, as might be surmised, impracticable. This temperature, $-273^{\circ}$, is of importance, however, since it is taken as the zero of a scale of temperature called the "Absolute" scale. The degrees on this scale are of the same value as those on the Centigrade scale, and Absolute temperature may be expressed on the Centigrade scale by subtracting $273^{\circ}$, while the reverse operation must be performed to change Centigrade temperatures into Absolute. Thus $365^{\circ}$ Abs. $=365-273=92^{\circ} \mathrm{C}$, and $-24^{\circ} \mathrm{C}$. $=-24+273=249^{\circ} \mathrm{Abs}$.

By the utilisation of the Absolute scale of temperature, Charles's Law may be expressed in a very simple form. Thus :

The volume of a given mass of gas is directly proportional to its Absolute temperature.

100. We will conclude this chapter by describing a method of solving problems in which changes of volume of a gas, caused by changes of both pressure and temperature, are involved.

A balloon with a capacity of $50,000 \mathrm{cu}$. $\mathrm{ft}$. is filled with coal-gas at a temperature of $18^{\circ}$ and under the atmospheric pressure of $765 \mathrm{~mm}$. How many cu. $\mathrm{ft}$. of gas will have escaped by the time it has risen to an 
altitude at which the temperature is $9^{\circ}$ and the pressure $680 \mathrm{~mm}$. ?

Transforming the Centigrade temperatures into Absolute temperatures, they become $291^{\circ}$ and $282^{\circ}$. Since the volumes of gas are proportional to these temperatures, the volume which $50,000 \mathrm{cu}$. ft. at $18^{\circ}$ will have at $9^{\circ}$ is $50,000 \times \frac{2}{2} \frac{2}{91} \mathrm{cu}$. ft.

This would be the volume, assuming that no pressure change were effected simultaneously with the alteration in temperature, and the pressure change must be allowed for by multiplying the volume, corrected for temperature as above, by $\frac{765}{680}$, as required by Boyle's Law. Thus the volume which the coal-gas would occupy at $9^{\circ}$ and $680 \mathrm{~mm}$. is

$$
50,000 \times \frac{282}{291} \times \frac{765}{680}=54,509 \mathrm{cu} . \mathrm{ft} .
$$

Assuming the capacity of the balloon to be unaffected by its ascension, it is evident that 54,509 $-50,000=4509 \mathrm{cu}$. ft. of gas must have escaped from it.

The above method, in which the changes of volume brought about by variations of temperature and pressure are considered consecutively, and the law governing each such change is directly invoked in finding the necessary correction in each case, is recommended in preference to methods involving the use of memorised formulae, in which mere substitution of the values of the quantities mentioned in the question for the various symbols in the formulae takes the place of reasoning based on knowledge of the properties of gaseous bodies. 


\section{QUESTIONS}

1. Devise and explain an experiment to show that, in determining the temperature of a body, misleading evidence may be obtained by trusting solely to one's bodily sensations as a guide.

2. To which state of matter do you consider the softened glass from which a thermometer bulb is blown to belong, and why?

3. Give reasons for the following observations made in Experiment 46 :

(a) The escape of bubbles through the coloured alcohol when the bulb is placed in warm water.

(b) The passage of the alcohol into the tube and bulb when the heating of the latter is discontinued.

(c) The filling of the bulb being almost complete after its second heating.

4. The average temperature of human blood in the living subject is $98.4^{\circ} \mathrm{F}$. Express this in degrees Centigrade and in degrees Réaumur.

5. The boiling-points of mercury and of air are respectively $357^{\circ} \mathrm{C}$. and $-192^{\circ} \mathrm{C}$. What are the corresponding temperatures on the Fahrenheit scale?

6. Suggest the probable reason for the statement that it is not easy to make a good cup of tea at the top of a high mountain.

7. Which has the higher temperature-a gallon or a pint of boiling water ? Support your opinion by writing out your arguments in favour of it.

8. A vessel containing hot water is placed under the receiver of an air-pump, and the pump put into action. What would you expect to happen, and why ?

9. In a room without a fire the fender seems to be colder than the door, and the door colder than the carpet. By what experiments would you test the accuracy or otherwise of these observations ?

10. At what temperature will the volume of a mass of air, which occupies 562 c.c. at $20^{\circ}$, become (a) 659 c.c. ; (b) 340 c.c. ?

11. A litre flask, fitted with a cork and delivery tube, is placed in a vessel and surrounded by melting ice, the end of the delivery tube being left open to the air. After ten minutes the end of the 
tube is placed under water beneath the mouth of a large jar filled with water, and the vessel containing the ice is heated. What volume of air at $20^{\circ}$ will be collected in the jar if the heating be continued till the flask is surrounded by boiling water? (The volume of the delivery tube may be neglected.)

12. $1 \mathrm{~g}$. of hydrogen at $0^{\circ}$ and $760 \mathrm{~mm}$. occupies $11 \cdot 127$ litres. What will be its volume $(a)$ at $132^{\circ}$ and $800 \mathrm{~mm}$. ; $(b)$ at $-132^{\circ}$ and $745 \mathrm{~mm}$. ?

\section{CHAPTER $\mathrm{X}$}

\section{HEAT AS A QUANTITY}

101. In the last chapter it was shown that a teacupful of hot water was as hot as a kettleful when neither quantity of water gave up heat to the other on being mixed. When preparing a hot bath, however, it would be of little service to add a teacupful of boiling water to each gallon of cold water used, while a bath of suitable temperature may well be prepared by putting in a kettleful of boiling water for each gallon of cold, supposing the kettle of average size. Although the teacup and kettle contain quantities of water which are equally hot, their contents do not possess equal heating power, or, in other words, the quantities of heat contained by the contents of the two vessels are not equal.

To test the above statement, we will perform

Experiment 52. Pour into a beaker 100 c.c. of water from a measuring cylinder. Into another beaker measure out 500 c.c. of water and take its temperature. Heat the water in the first beaker, with constant 
stirring, till it has attained a temperature of $60^{\circ} \mathrm{C}$., as shown by a thermometer. Pour the hot water quickly into the beaker containing the larger quantity, stir well, and note the highest temperature recorded by a thermometer placed in the mixture.

Next heat 500 c.c. of water to $60^{\circ} \mathrm{C}$. and pour this into 500 c.c. of cold water at as nearly as possible the same temperature as that previously used. Stir and again note the highest reading given by a thermometer immersed in the mixture.

The temperature of the latter mixture will be observed to be considerably higher than that of the former, thus showing that the larger quantity of hot water gave up more heat to the cold water than did the other, although both quantities of water were at the same temperature originally.

From the above considerations, it is clear that the temperature of a body and the quantity of heat contained in the body are quite different things. The temperature is entirely independent of the mass of the body, while the heat-content is very materially affected thereby.

Experiment 53. Pour 100 c.c. of cold water into each of two beakers, and note the temperature in each case. Weigh a lump of lead (or other convenient metal) and place it in a third beaker. Pour into this beaker as many c.c. of water as the number of grams in the weight of the lead. Heat the beaker and its contents to a temperature of $60^{\circ} \mathrm{C}$., with constant stirring. Remove the lead, quickly wipe it dry, and place it in one of the beakers containing cold water previously prepared. Stir well and note the temperature reached by the water. Pour the warm water into the second supply of cold water, stir, and note the highest temperature attained. 
It will be found that the reading in the case of the lead is much lower than that obtained with the hot water. Repeat the experiment with lumps of different substances, such as a large glass stopper, a $100 \mathrm{~g}$. weight, a large flint, and a quantity of mercury, using in each case a mass of hot water equal to that of the body. These will produce varying increments of temperature in the cold water, but each will cause a smaller increase than is produced by an equal mass of hot water.

As stated in the last chapter, temperature is independent of the material of which a body is made. The above experiment indicates that the quantity of heat contained in different bodies of equal mass at the same temperature is not the same, i.e. that the quantity of heat is not independent of the material composing a body. Further it appears that a given mass of water at a given temperature contains more heat than any of the other substances tested.

102. Since heat is a quantity, it may be measured, given a suitable unit of measurement. The unit commonly adopted is known as the calorie. This unit has been variously defined, but for our purposes it will be sufficient to ignore the small variations of value consequent on the adoption of one or other definition and to state that "a calorie is the quantity of heat required to raise the temperature of one gram of water one degree Centigrade". To heat 120 g. (or 120 c.c., if no account be taken of the small variations of the density of water with temperature) of water from $15^{\circ} \mathrm{C}$. to $87^{\circ} \mathrm{C}$. will therefore require that energy be supplied to the water in the form of heat to the extent of $\{120 \times(87-15)\}=8640$ calories. In cooling from $87^{\circ} \mathrm{C}$. to $15^{\circ} \mathrm{C}$., the same amount of water will supply H. D. $\mathrm{s}$. 
the same quantity of heat, viz. 8640 calories, to its surroundings.

103. We may easily find by calculation the temperature of the water resulting from the admixture of two quantities of water at different temperatures, if we assume that all the heat supplied by the water at the higher temperature is applied to raising the temperature of the colder water. Suppose that, in Experiment 52, 100 c.c. of water at $60^{\circ} \mathrm{C}$. were mixed with 500 c.c. at $15^{\circ} \mathrm{C}$. Let $n^{\circ} \mathrm{C}$. be the temperature attained. by the mixture. We have

Heat given out by hot water $=100 \times(60-n)$ calories ;

Heat received by cold water $=500 \times(n-15)$ calories.

On our assumption, these two quantities must be equal, i.e.

$$
100 \times(60-n)=500 \times(n-15) .
$$

Solving this as a simple equation, we have

$$
600 n=13,500, \quad \text { or } n=22 \cdot 5^{\circ} \mathrm{C} \text {. }
$$

104. From Experiment 53 we learnt that different substances have different capacities for heat, since bodies of different materials gave out unequal quantities of heat while cooling. The capacity of a given body for heat is called its " thermal capacity" and is measured by the quantity of heat necessary to raise its temperature $1^{\circ} \mathrm{C}$. Thus, if $30 \mathrm{~g}$. of mercury have to be supplied with 1 unit of heat in order to cause the temperature of the liquid to rise $1^{\circ} \mathrm{C}$., the thermal capacity of the $30 \mathrm{~g}$. is said to be 1 . When the thermal capacity of unit mass is considered, a new term is introduced, this quantity being known as the "specific heat" of the substance dealt with. Since $1 \mathrm{~g}$. of water requires 
1 calorie to be supplied to raise its temperature by $1^{\circ} \mathrm{C}$., the specific heat of water is $1.1 \mathrm{~g}$. of mercury only requires 0.033 heat-units to raise its temperature by the same amount, hence the specific heat of mercury is 0.033 . The specific heats of some common substances are given in the table below :

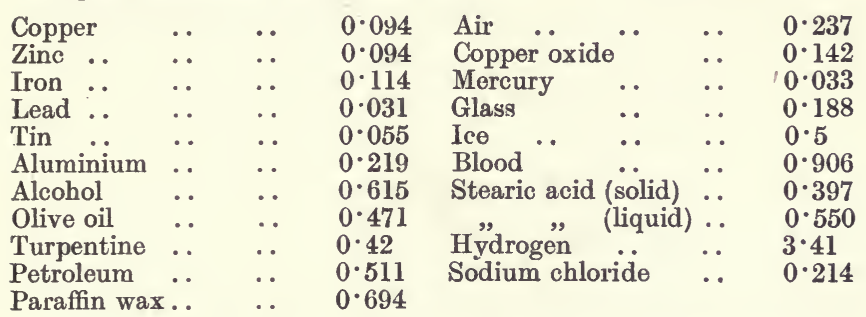

Note. In all cases where the scale of temperature is not definitely mentioned in the following pages, the Centigrade scale is to be understood.

105. The method of working simple examples in which the specific heats of the substances used must be considered is shown below.

Example 1. Suppose an iron cylinder weighing $100 \mathrm{~g}$. to be used in Experiment 53. What will be the temperature of the water after the iron has been. placed in it?

Let this temperature be denoted by $n$.

In cooling from $60^{\circ}$ to $n^{\circ}$, the iron will lose $100 \times(60-n) \times 0.114$ calories. To raise the temperature of the water from, say $15^{\circ} \mathrm{C}$., to $n^{\circ} \mathrm{C}$., will require $100 \times(n-15)$ calories to be supplied. Supposing no heat to be lost in heating the beaker and its surroundings, these two quantities of heat must be equal, i.e.

$$
100 \times(60-n) \times 0.114=100 \times(n-15) .
$$

Solving this as a simple equation in $n$, we have $111 \cdot 4 n=2184$, or $n=19 \cdot 6^{\circ} \mathrm{C}$. 
106. In actual practice it is, of course, almost impossible to prevent the loss of some of the heat supplied by the hot body, and, in any case, when a liquid is heated by it, a portion of the heat is used in raising the temperature of the containing vessel. Allowance must, therefore, be made for this in calculation. This is usually done by finding the thermal capacity of the vessel by multiplying the weight of the vessel by the specific heat of the substance of which it is made. Thus, suppose that the beaker containing the cold water in Experiment 53 weighs $25 \mathrm{~g}$. Its thermal capacity will be $25 \times 0 \cdot 188=4 \cdot 7$ calories. That is to say, to raise its temperature by a given amount will require the supply of as much heat as is required to raise the temperature of $4 \cdot 7 \mathrm{~g}$. of water by the same amount. Looked at from this point of view, the thermal capacity of the vessel is called its "waterequivalent".

Example 2. What will be the temperature attained by the water in Experiment 53, if $100 \mathrm{~g}$. of iron at $60^{\circ} \mathrm{C}$. be used, and the water, at $15^{\circ} \mathrm{C}$., be contained in a beaker weighing $25 \mathrm{~g}$. ?

The beaker and cold water will be equivalent to a single quantity of water weighing $100+4 \cdot 7=104 \cdot 7 \mathrm{~g}$. Our equation will hence become

$$
\begin{gathered}
100 \times(60-n) \times 0.114=104.7 \times(n-15), \\
\text { or } \quad 116.1 n=2254.5, \text { i.e. } n=19.4^{\circ} \mathrm{C} .
\end{gathered}
$$

Example 3. Determine the specific heat of zinc from the following data:

Weight of zinc

$$
\begin{array}{ccccr}
\text { Weight of zinc } & \ldots & \ldots & \ldots & 300 \mathrm{~g} . \\
,, \quad \text { beaker } & \ldots & \ldots & \ldots & 56 \mathrm{~g} . \\
", \quad \text { ", and } & \text { cold } & \text { water } & \ldots & 515 \mathrm{~g} . \\
\text { Temperature of zinc } & \ldots & \ldots & \ldots & 100^{\circ}
\end{array}
$$$$
\text { , } \quad, \quad \text { and cold water ... } 515 \mathrm{~g} \text {. }
$$ 
Temperature of water .. $\quad \ldots \quad \ldots \quad 10^{\circ}$ , after putting in zinc $15 \cdot 2^{\circ}$

$\begin{array}{lllll}\text { Specific heat of glass } & \ldots & \ldots & \ldots & 0.188\end{array}$

The water-equivalent of the beaker is $56 \times 0.188$ $=10.5$. Let $S$ denote the specific heat of zinc. Then Heat supplied by zinc $=300 \times S \times(100-15 \cdot 2)$ cal .

Heat received by water and beaker

$$
=(515-56+10.5) \times(15 \cdot 2-10) \mathrm{cal} .
$$

Equating these quantities, we have

$$
300 \times 84 \cdot 8 S=469 \cdot 5 \times 5 \cdot 2 \text {, or } 25,440 S=2441 \cdot 4,
$$

whence $\mathbf{S}=0.096$.

107. Experiment 54. Weigh a porcelain dish. In it place a few small lumps of ice, dried by wiping with a duster. Quickly weigh the porcelain dish and ice. Heat the dish over a small Bunsen flame, stirring the ice and the water formed, and noting how long it takes to melt the ice completely. Note the temperature from time to time, and when the ice has all become water, continue the heating till the water has reached a temperature of $20^{\circ}$. Make a note of the time required to raise the temperature of the water from $0^{\circ}$ to $20^{\circ}$. Again weigh the dish and its contents.

Some of the observations made will be

(a) That it takes about four times as long to change the ice at $0^{\circ}$ into water at the same temperature as it does to heat the water formed from $0^{\circ}$ to $20^{\circ}$.

(b) That the water formed by the melting of the ice has the same weight as the ice.

Suppose the weight of the ice to be $58 \mathrm{~g}$. In raising the temperature of the water formed from 
$0^{\circ}$ to $20^{\circ}, 58 \times 20=1160$ calories must be supplied. Since it took about four times as long to melt the ice, using the same source of heat, the quantity of heat supplied during the melting must have been about $4 \times 1160=4640$ calories. Hence to melt $1 \mathrm{~g}$. of ice, without producing any rise of temperature, $4640 \div 58$ $=80$ calories have been used. This quantity of heat, used in merely changing the physical state of the ice, is said to be "latent" in the water formed, and the "latent heat of fusion of ice" is said to be 80 . The word "latent" means "hidden", and, if we performed the above experiment in the reverse order, the suitability of the name would be evident. It is not easy to obtain measurements with any degree of accuracy in doing this, but a rough idea may be obtained by performing

Experiment 55. Pound up some ice in a mortar. Place a layer of this crushed ice at the bottom of a large beaker, and cover it with a layer of ordinary salt, using roughly about half as much salt as ice. Put into the beaker two more layers of each substance. Place a smaller beaker upon the top of the last layer, and surround this beaker with alternate layers of the ice and salt till the level of the mixture reaches the lip of the small beaker. Press the mixture down firmly. Remove the small beaker, wipe it dry, and half fill it with water. Warm the water, if necessary, to $20^{\circ}$, and then replace the beaker. Keeping it constantly stirred with a thermometer, note the time taken to cause the temperature of the water to fall to $0^{\circ}$. The mixture of ice and salt is much colder than would be the case if ice alone were used, and, if the thermometer be allowed to remain in the water, ice will be formed, but no further fall of temperature 
will be noted till after all the water in the smaller beaker has become ice. By examining the beaker at intervals, the time taken to -bring about the change of the water at $0^{\circ}$ into ice at $0^{\circ}$, may be approximately found. It will be noticed that it is about four times as long as was required to lower the temperature of the water from $20^{\circ}$ to $0^{\circ}$. If the inner beaker be left in the mixture for a further interval, it will be noted that the ice will continue to give up heat to the cold mixture of ice and salt, as shown by its temperature falling below $0^{\circ}$.

In the above experiment, the heat given out by the water in cooling causes some of the ice in the outer vessel to melt, and we have evidence that this heat is actually given out in the fall of temperature experienced by the water. The water, in changing to ice, continues to give out heat-its latent heat-and melts more of the surrounding ice, but we have no evidence of this registered by the thermometer. That the supply of heat from the inner to the outer vessel is a continuous process, and does not stop when the water reaches $0^{\circ}$, may be inferred from the fact that as soon as the whole of the liquid has become solid, further decrease in its temperature is recorded by the thermometer.

108. Experiment 56. Into a porcelain evaporating dish pour 20 c.c. of water, and take its temperature. Heat the dish over a small flame and note the time taken to raise the temperature of the water to $100^{\circ}$. Continue the heating with the same flame until the water has been completely turned into steam. Make a note of the time required to effect this change, and take the temperature of the water from time to time while it is proceeding. 
If the water be at about the average temperature of water from the tap, say $15^{\circ}$, before the heating is started, it will be found that it requires about six times as long to turn the water at $100^{\circ}$ into steam as it does to raise its temperature from $15^{\circ}$ to $100^{\circ}$. Since $20 \times(100-15)=1700$ calories are needed to effect the latter change, it follows that some 10,200 calories are used up in merely changing the physical state of the water. For every gram of water over 500 calories are rendered "latent" during "the vaporisation. A more exact value is $\mathbf{5 3 6}$.

Note. It is possible, of course, that the steam is hotter than the boiling water, and that some of the heat is used in raising the temperature of the steam. To test this, repeat Experiment 48, and note whether the temperature of the steam evolved from the boiling water shows any increase of temperature during 10 minutes' boiling.

Other substances possess latent heats of fusion and vaporisation, and some examples are given in the subjoined table :

Substance

Water. .

Mercury

Nitrate of soda

Sulphur $\quad$. $\quad$..

Alcohol .. ..

Ether.. $\quad . . \quad$..

Turpentine .. ..
L. H. of Fusion

80

$2 \cdot 82$

63

$9 \cdot 4$
L. H. of Vaporisation

536

62

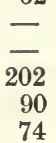

109. To determine the latent heats of fusion and vaporisation of water with a greater approach to accuracy than was possible in the experiments already described, the following experiments should next be performed. 
Experiment 57. Arrange two beakers as shown in Fig. 51. The space between the vessels is loosely packed with cotton-wool. (The reason for this precaution will be understood after reading the chapter on methods of transmission of heat.) Weigh the inner beaker, and introduce into it enough water to fill it about half full. Weigh the beaker and water.

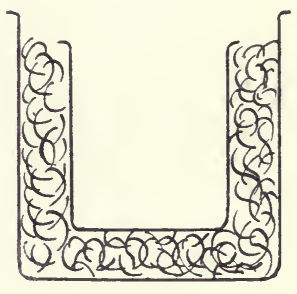

Fig. 51 . Place a thermometer in the water and find its temperature. By gentle heating, raise its temperature about $5^{\circ}$. Replace it inside the larger beaker, keeping the thermometer in its place whilè doing so. Wipe a small lump of ice with a duster, note the exact temperature recorded by the thermometer, and quickly drop in the lump of dried ice. Stir well with the thermometer until the ice is almost entirely melted. Note the temperature. If it has fallen about $10^{\circ}$, continue stirring until the ice has all melted, carefully noting the lowest temperature registered. If the decrease is less than $10^{\circ}$, put in another piece of dry ice, and repeat this till the amount of ice added is sufficient to cause the temperature to fall about $10^{\circ}$. Make a careful determination of the lowest temperature recorded. Finally weigh the inner beaker and its contents.

In an actual determination, the following observations were made :

Weight of beaker $\quad . \quad \quad \ldots \quad \quad \ldots \quad \quad \ldots \quad 84 \cdot 6 \mathrm{~g}$.

$$
\text { and water } \quad \ldots \quad \ldots 308.9 \mathrm{~g} \text {. }
$$

" - , after adding ice $335 \cdot 6 \mathrm{~g}$. 
Original temperature of water $\quad \ldots \quad \ldots \quad 21^{\circ}$ Final

Let the latent heat of fusion of ice be denoted by $L$.

Water-equivalent of beaker $=84 \cdot 6 \times 0 \cdot 188=15.8$.

Heat supplied by water and beaker

$$
=(224 \cdot 3+15 \cdot 8) \times(21-11) \mathrm{cal} .
$$

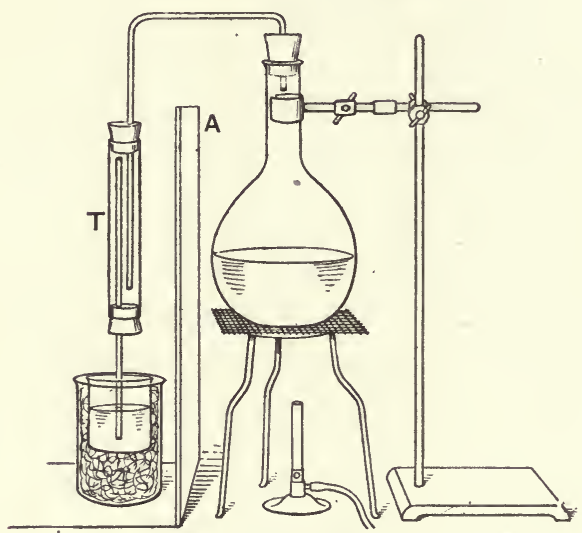

Fig. 52.

Heat rendered latent during fusion

$$
=(335 \cdot 6-308 \cdot 9) \times L \text { cal. }
$$

Heat needed to raise temperature of water formed by fusion of the ice from $0^{\circ}$ to $11^{\circ}$

$$
=\left(335 \cdot 6-308^{\circ} 9\right) \times 11 \mathrm{cal} \text {. }
$$

- Equating the first quantity of heat to the other two, we have $26 \cdot 7 L=2107 \cdot 3$, or $\mathrm{L}=79$. 
In this experiment the result obtained was extremely close to that generally given as the most probably correct value, but this is not always the case with experiments done as described, since small errors are practically unavoidable, and, further, the specific heat of the glass is only an average value, and that of the glass actually used may be different to the extent of over $10 \%$. With care values for the latent heat may be found which will lie between 75 and 80 .

Experiment 58. Arrange the apparatus illustrated in Fig. 52. $A$ is a screen of wood or asbestos to prevent direct heat from the flame and the heated flask from reaching the beaker and its contents. $T$ is a "steamtrap". Its function is to prevent as far as possible the entrance of hot water, formed by the condensation of a portion of the steam, into the water contained in the inner beaker. . Weigh this beaker empty. Half fill it with water in which sufficient ice has been melted to bring it to a temperature of about $5^{\circ}$. Weigh the beaker and cold water, and replace it in the larger beaker. As in the last experiment, the space between the beakers is packed with cotton-wool. Heat the water in the flask to boiling-point, and, after steam has been allowed to escape from the lower end of the trap for about two minutes, put the lower end of the trap in the water in the beaker, noting the exact temperature of the water at the instant of introducing the tube. Let the steam continue to pass through the cold water till the temperature of the latter has risen about $20^{\circ}$. Remove the end of the trap, stir the water well with the thermometer, and record the highest temperature registered. Weigh the beaker and contained water.

The latent heat of vaporisation of water was 
obtained in an actual determination by the above method as follows :

Weight of beaker

" $\quad$ " and cold water $\quad . \quad \ldots 341 \cdot 8 \mathrm{~g}$.

" $\quad, \quad$ water at end of expt. . $351^{\circ} 6 \mathrm{~g}$.

Temperature of water at beginning of expt. . $\quad 5 \cdot 5^{\circ}$

$" \quad$ end $" \quad . \quad 26.9^{\circ}$

If $L$ denote the latent heat, we have

Heat supplied by steam in condensing to water

$$
=(351 \cdot 6-341 \cdot 8) L \text { cal. }
$$

Heat given out by water formed from the steam in cooling to $26^{\circ} 9^{\circ}$

$$
=(351 \cdot 6-341 \cdot 8) \times(100-26 \cdot 9) \mathrm{cal} .
$$

Heat received by the cold water and beaker

$$
=(341: 8-84 \cdot 6+15 \cdot 8) \times(26 \cdot 9-5 \cdot 5) \mathrm{cal} \text {. }
$$

The last quantity of heat is equal to the sum of the other two, i.e.

$$
273 \times 21 \cdot 4=9 \cdot 8 L+9 \cdot 8 \times 73 \cdot 1 .
$$

Solving this equation in $L$, we have

$$
9 \cdot 8 L=5125 \cdot 8, \text { or } \mathrm{L}=523 .
$$

The results obtained are always somewhat low, since it is practically impossible to assure that only "dry" steam (i.e. steam containing no condensation water whatever) shall pass into the cold water.

110. From the working out of the results given in the last two experiments, it will be fairly easy to devise methods of solving problems in which latent heats are involved.

Instead of the glass beakers used in the experiments on specific and latent heats, specially made copper 
vessels may be substituted, but these are not necessary unless a fairly high degree of accuracy is desired in the results obtained. Vessels such as the beakers and copper cans, when made use of in the measurement of quantities of heat, are termed "calorimeters" (Latin, calor, heat).

111. A striking experiment showing the great amount of heat absorbed during the evaporation of a liquid may be performed with the readily volatile and extremely inflammable liquid, ether. This experiment must not on any account be attempted in the neighbourhood of flames, and is best done by, or under the immediate supervision of, a teacher.

Experiment 59. Into a small beaker pour enough ether to fill it one-quarter full. Let about six drops of water fall on the middle of one face of a small wooden block. Put the beaker on the block in contact with the water. Blow air through a glass tube attached to a bellows, worked by hand or by the foot, by means of rubber tubing, the end of the tube being made to dip under the surface of the ether. The rapid bubbling thus produced causes a large surface of ether to be exposed, and greatly increases the rate at which the liquid evaporates. Since no heat is supplied from an outside source, that required for the conversion of the ether from the liquid to the gaseous state must be taken from the ether and the bodies in contact with the ether. As a result, these bodies, i.e. the beaker, water, and block, have their temperatures reduced considerably. By steady blowing it is easy to lower the temperature of the water to $0^{\circ}$, and continuance of the operation causes the formation of ice, the beaker being frozen to the wooden block. 
112. In the table of specific gravities given in Chapter IV, that of ice is shown as 0.917 , i.e. ice is less dense than water, a statement corroborated by the familiar fact that ice floats on water. Hence, during the melting of a given mass of ice, a contraction of volume occurs. This phenomenon may be easily shown in the manner described in the next experiment.

Experiment 60. Break up some ice intopieces small enough to be placed in a flask of about 500 c.c. capacity. Fill such a flask with the ice as completely as possible, and add enough water, coloured with litmus and cooled by dropping in a few pieces of the ice, to entirely fill the spaces between the

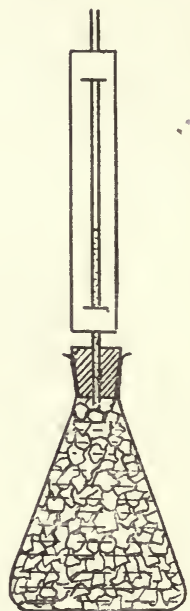

Fig. 53. lumps of ice and the neck of the flask. Fit the flask with a well-fitting rubber stopper, carrying a long piece of glass tubing and a paper scale as illustrated in Fig. 53. In pushing the stopper home, some of the water will be forced up the tube. Mark the level to which it reaches on the scale. Allow the apparatus to stand in a warm room. The consequent melting of some of the ice will be accompanied by a steady fall in the level of the liquid column in the tube, showing that the volume of the resulting water is less than that of the ice from which it is formed.

113. During the increase of volume attendant upon the change of water into ice, great force is exerted, if the liquid be contained in a completely closed vessel. The water-pipes within an ordinary house constitute such a closed vessel when all taps are turned off. In 
very cold weather the water in the pipes may be frozen, when the strain upon the material of the pipes is usually sufficient to burst them, some of the water escaping from the rent in the pipe and immediately freezing, thus sealing up the pipe. As the weather becomes warmer, this ice-seal melts, and the water flowing through the pipe escapes at the point of bursting - commonly to be found in that portion of the pipes situated near the top of the building - causing much annoyance and loss to the unfortunate householder. It should be noticed that the burst occurs during frosty weather, but that the awkward consequences are not felt till a thaw sets in. This has given rise to a more or less prevalent and entirely mistaken impression that it is the thawing of the water which causes the bursting of the pipes.

114. The augmentation of volume taking place when water becomes ice is but small when compared with that produced when it assumes the gaseous condition. 1 c.c. of water, boiling under atmospheric pressure, gives rise to over 1600 c.c. of steam at the same pressure. It is not easy to devise a simple experiment in illustration of this, since the prevention of condensation of the steam presents difficulty. From another point of view most of us are familiar with this phenomenon, for all work done by steam-engines depends upon the great pressure exerted by the steam escaping from boiling water, this pressure being due to the effort of the steam to expand. Should this pressure become too great for the material of the boiler of the engine to support, the boiler bursts, unless by the use of suitable safety-valves some portion of the steam is allowed to escape, and the pressure thereby reduced. 
115. A corresponding diminution of volume occurs when steam condenses, and this is taken advantage of in a simple method whereby an approximate determination of the specific gravity of air may be effected.

Experiment 61. Fit a roundbottomed flask of about 500 c.c. capacity with a rubber stopper, bored to receive a short glass tube. To the other end of this tube attach, by means of stout rubber tubing, a second short length of glass tube. Fix a small screw clamp on the rubber tubing. The general arrangement of the apparatus is shown in Fig. 54. The wire shown is for suspending the apparatus from the hook at the end of the arm of a

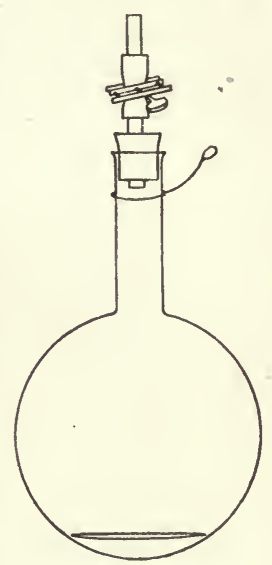

Fig. 54. balance while weighing. Pour about 50 c.c. of distilled water into the flask. Heat this to boiling-point, and allow the heating to continue till most of the water has boiled away. In this manner practically all the air is displaced by steam. While still boiling, clamp the rubber tube, and at once remove the source of heat. Let the flask cool and then weigh it carefully. Next open the clip gradually. The greater part of the steam has condensed during the cooling and thus produced a considerable decrease of pressure within the flask, so that the opening of the clip is attended by an inrush of air from the room until the pressure inside the flask has reached atmospheric pressure. When no more air enters, weigh the apparatus again. The gain in weight will be the weight of the air which has entered the flask. To find the volume 
of this air, pour out the water left in the flask into a small measuring cylinder and observe its volume. Next fill the flask to the level of the bottom edge of the stopper with water, and pour this water into a larger measuring vessel. The volume of the water thus poured out, minus that of the water left in the flask, is the volume of the air which passed into the flask.

The specific gravity of air may be found by dividing the weight of the air by the weight of the corresponding volume of water, as previously explained.

Knowing the specific gravity of air, we may determine those of other gases by filling a vessel of known capacity with the gas whose specific gravity is desired, weighing it, and also weighing the same vessel filled with air. As an example of this, find the specific gravity of carbon dioxide as in the following experiment.

Experiment 62. Find the volume of a flask as indicated in the previous experiment. Weigh the flask, which should be quite clean and dry, a well-fitting cork being placed in the neck. Fill the flask with carbon dioxide by passing the gas from a generator (see Fig. 98) in which the materials used are marble and hydrochloric acid. The gas should be dried by passage through a drying tube containing calcium chloride before it is allowed to enter the flask. The flask should be maintained in an upright position, and the gas should be passed in for at least 10 minutes in a steady stream with the end of the delivery tube almost touching the bottom of the flask. Slowly withdraw the delivery tube while the gas is still issuing from it, and re-cork the flask. Weigh flask and gas carefully.

Subtract the weight of the flask filled with air from its weight when filled with carbon dioxide. The

H. D. S. 
difference will be the excess of the weight of the gas over that of the air. Find the weight of the air by multiplying the volume of the flask in cubic centimetres by 0.001293 . (1 c.c. of air weighs $0.001293 \mathrm{~g}$. at $0^{\circ} \mathrm{C}$. and $760 \mathrm{~mm}$. bar.) Add this to the difference of weight as found above, and the sum will be the weight of the carbon dioxide. The specific gravity of the carbon dioxide is then readily found by the method given for air above.

\section{Exercise for Student.}

Suggest any sources of error in the methods of Experiments 61 and 62 .

116. In Experiment 48 a method of checking the accuracy of the position of the boiling-point of water, as marked on a mercury thermometer, was described. It was directed that the bulb of the instrument should be placed in the steam from boiling water and the reason for this direction will be next considered.

ExPERIMent 63. Using similar apparatus to that shown in Fig. 44, determine the temperatures of the steam arising from distilled water, tap-water, and $10 \%$ and $20 \%$ solutions of common salt, i.e. solutions containing $10 \mathrm{~g}$. and $20 \mathrm{~g}$. of salt respectively in every 100 c.c. of solution. Each liquid should be boiled in turn in the same flask and heated by the same source of heat, while all temperatures registered should be taken from the same thermometer.

After recording the temperature of the steam in each case, lower the thermometer into the boiling liquid and note the temperature shown, together with any variations which may occur as the period of boiling is prolonged. 
In the case of the distilled water, it will probably be found that the steam temperature differs but little from that of the boiling water, while any variations are temporary in character, no marked rise nor fall being noted. It is likely, however, that the thermometer, when plunged in the tap-water, will indicate that it is slightly hotter than the steam. Small variations of temperature may be observed, but, as in the previous case, no indication of any definite rise nor fall. The salt solutions will provide much more strongly marked evidence of the difference of temperature between the liquid and the steam immediately over it than that afforded by the tap-water, and the water will, in each case, be the hotter body. As the boiling is continued, the temperature registered will slowly increase to a maximum of about $108^{\circ}$, provided the period of heating be long enough. On comparing the four steam temperatures very little difference will be found between them. This observation may be confirmed by treating other solutions of substances in water similarly, provided that the solutions be not too concentrated. With very strong solutions the temperature of the escaping steam is distinctly higher than that of the steam from pure water.

The general deduction from the above observations is that

The presence of a substance in solution in water raises the temperature at which the liquid boils, while the temperature of the steam produced is but little affected, except with very strong solutions.

\section{Exercise for Student.}

Explain the slow rise of temperature produced when the salt solutions are kept boiling for some time. 
117. Dissolved substances also produce an effect upon the freezing-point of the liquid in which they are dissolved, but the alteration is of an opposite character, the temperature of freezing being lowered by the presence of the dissolved matter, the magnitude of the decrease being proportional to the strength of the solution.

Experiment 64. Surround a test-tube containing a little water with a freezing-mixture of ice and salt as described in Experiment 55. Insert a thermometer in the water and read the temperature at which the water freezes, as shown by the mercury becoming steady at a certain level. Using the same thermometer, find the temperatures at which $2 \%$ and $5 \%$ solutions of salt become frozen. Compare your observations with the statement given above.

118. When solid ice becomes liquid water, heat is absorbed. As will be shown later, the dissolving of a solid substance in a liquid may also be attended by a similar absorption of heat, and this action is fairly general. The cause of the production of a very low temperature by the admixture of ice and salt may now be readily grasped. Some of the salt dissolves in the water adhering to the ice, and thus a slight lowering of temperature is caused. A further fall is occasioned by the dissolving of part of the ice in the newly-formed salt solution, attended by the disappearance of the amount of heat rendered "latent" by this process. The continued operation of these two causes brings about a fall of temperature sufficient to make the mixture of great value as a means of producing cold. A thorough admixture of snow with one-third of its weight of salt will effect a lowering of temperature to $-21^{\circ}$. 


\section{QUESTIONS}

1. What will be the temperature of the liquid resulting from the pouring of $2 \frac{1}{2}$ pints of water at $200^{\circ} \mathrm{F}$. into a gallon of water at $65^{\circ} \mathrm{F}$. ?

2. How much water at $98^{\circ} \mathrm{C}$. must be added to 5 kilograms of water at $16^{\circ} \mathrm{C}$. in order that the temperature of the resulting mixture may be $63^{\circ} \mathrm{C}$. ?

3. Which is hotter-a pound of water at $80^{\circ} \mathrm{C}$. or 20 pounds of mercury at $90^{\circ} \mathrm{C}$., and which will cause the greater rise in the temperature of a kilogram of water at $25^{\circ} \mathrm{C}$., when mixed with the latter?

4. $50 \mathrm{~g}$. of magnesium (sp. heat, $0 \cdot 245$ ) at $15^{\circ} \mathrm{C}$. are dropped into $100 \mathrm{~g}$. of water at $30^{\circ} \mathrm{C}$. What is the highest temperature to which the magnesium can be raised by this treatment?

5. Find the specific heat of lead from the following data:

$\begin{array}{lccc}\text { Weight of leaden shot used } & \ldots & \ldots & 200 \mathrm{~g} \text {. } \\ \text { Weight of water in calorimeter } & \ldots & \ldots & 100 \mathrm{~g} \text {. } \\ \text { Temperature of shot } & \ldots & \ldots & 100^{\circ} \mathrm{C} \text {. } \\ \text { Initial temperature of water } & \ldots & \ldots & 9 \cdot 6^{\circ} \mathrm{C} \text {. } \\ \text { Final temperature of water } & \ldots & \ldots & 14 \cdot 9^{\circ} \mathrm{C} \text {. }\end{array}$

6. A flask containing a litre of mercury at $10^{\circ}$ is placed in a vessel of boiling water and allowed to remain till it has attained the temperature of the water. How much heat is supplied to the mercury by the water? If the mercury be caused to give up the heat thus received to a litre of water at $10^{\circ}$, by how much will the temperature of this volume of water be raised ?

7. Pieces of platinum and aluminium foil of equal weight and area are both rendered red-hot, and then allowed to cool. Why may the platinum be handled with impunity with the fingers sooner than the aluminium ?

8. $500 \mathrm{~g}$. of mercury at $100^{\circ} \mathrm{C}$. are poured into a hole in a large block of dry ice. How many grams of water will be formed during the time taken by the temperature of the mercury to sink to $0^{\circ} \mathrm{C}$. ?

9. $200 \mathrm{~g}$. of ice are melted by dropping the ice into a vessel containing $400 \mathrm{~g}$. of water at $45^{\circ} \mathrm{C}$. The temperature of the water falls to $5^{\circ} \mathrm{C}$. Find the water-equivalent of the vessel. 
10. By how much will the temperature of a litre of ice-cold water be raised by the condensation of $100 \mathrm{~g}$. of steam in it?

11. Why is it advantageous to put some salt in the water with which doorsteps are cleaned in frosty weather ?

12. How many calories must be supplied to melt a block of ice which measures $25 \mathrm{~cm} . \times 15 \mathrm{~cm} . \times 10^{\circ} 8 \mathrm{~cm}$. ?

13. Describe fully an experiment in which it is shown that the liquefaction of a solid is attended by absorption of heat, and another in which the solidification of a liquid is shown to cause the evolution of heat.

14. A stout glass bottle is completely filled with water and securely stoppered. It is then placed in the open air on a day when the thermometer registers $23^{\circ} \mathrm{F}$. Describe what you would expect to happen.

15. A lump of ice is thrown into a vessel containing a quantity of alcohol at $-25^{\circ} \mathrm{C}$. What happens ? in it.

16. Suggest two reasons for tea being cooled by dissolving sugar

17. Of what advantage to mankind is the fact that water gives out heat while freezing ?

18. Most substances occupy a greater volume when in the liquid state than when solid. What useful purpose is served in nature by the peculiar behaviour of water in this respect?

\section{CHAPTER XI}

\section{THE PROPAGATION OF HEAT}

119. In the preceding chapters on heat we have frequently spoken of heat being transmitted from one body to another, and the experiments performed have afforded evidence that this transmission actually takes place. We will next take up the question of the various methods by which heat moves from one body to another, or from one part of a body to some other part. 
120. From everyday experience we know that the end of a poker placed in a hot fire soon becomes redhot, and that the other end often reaches such a high temperature that it is too hot to hold, although the hand may be held for some time at a distance from the fire equal to the length of that portion of the poker outside the fire without becoming uncomfortably hot. Heat evidently travels along the material of the poker in such a way that the end becomes hotter than the air situated at the same distance from the fire.

EXPERIMENT 65. Obtain two thin rods of different metals of similar diameter. A knitting-needle and a length of stout copper wire will serve if no better materials be available. Support the rods on a sheet of asbestos millboard placed upon the top of a tripod stand in such a way that one end of each may be heated by the same Bunsen flame. Arrange that the heating shall be as nearly as is feasible the same for each rod. Test the temperatures attained by the other ends of the rods from time to time by rough comparative methods such as the following:

(a) By touching the ends with the fingers, find out which first becomes too hot to be held with comfort.

(b) By placing the end of a wax-taper at each end in turn, find which first reaches the temperature at which the wax melts.

(c) Place the head of a match at each of the ends to discover whether either is hot enough to ignite the match. If neither becomes sufficiently hot to do this, move the match-head along the rods to find that point on each which has become hot enough to bring this about.

If rods of copper and iron be made use of, as suggested, the copper will be observed to become hot much 
more quickly than the iron, and the end of the copper rod further from the source of heat reaches a higher temperature than does the corresponding portion of the iron. A more elaborate experiment may be done if Ingenhaus's apparatus be available.

Experiment 66. The apparatus referred to above consists of a boiler of a rectangular shape, fitted with corks along one vertical face, in which rods of different metals but of equal length and diameter may be inserted. The rods project to similar distances within the boiler. The portions of the rods outside the

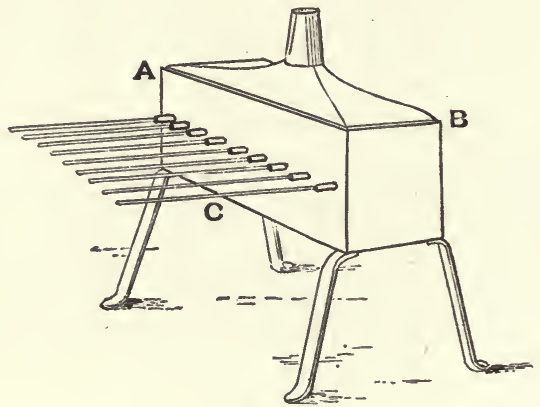

Fig. 55 .

boiler are coated with paraffin wax by melting some wax and applying it to the rods with a small brush. Boiling water is poured into the heater and the rate at which the wax melts is observed for each rod. When no more wax is melted by the heat transmitted the distances from the face of the boiler to the various points on the rods at which the wax is still solid are measured. The greater the distance, the better the material transmits heat through its mass.

121. This method of propagation of heat is termed "conduction" and it is by conduction that heat passes 
from one part of a solid body to another. No motion of the body as a whole or of any parts of it as seen by the eye takes place, the transmission being effected by the molecules of the substances nearest the heating source giving up part of the heat supplied by the heating agent to their neighbours, which again pass on part of the heat thus received in a similar fashion. The best conductors are the metals, and among these the list is headed by silver, closely followed by copper. Brass, iron, lead; and bismuth come next in the order of naming. The conducting power of silver is over 50 times that of bismuth. Compared with the metals, other solids are relatively poor conductors. This may be shown practically by repeating Experiment 65 with a stick of slate-pencil and a metal rod of equal diameter. The end of the pencil will be found to remain cool enough to be handled long after the metal rod is too hot to touch.

122. Conduction takes place also in liquid bodies, but all liquids come under the heading of bad conductors. That this is so in the case of water may be illustrated by a striking experiment.

ExPERIMENT 67. Surround a small lump of ice with copper or iron gauze in sufficient quantity to make the ice sink when placed in water. Drop the ice thus prepared into a large test-tube, and nearly fill the tube with water. Holding the tube near the middle of its length, heat the water in the upper portion in a flame. The water in this part of the test-tube may be raised to boiling-point before

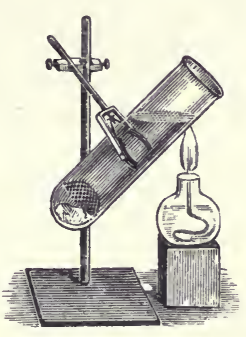

Fig. 56. the ice at the bottom of the tube has melted. 
123. Gases exhibit conducting power, but to an extremely limited extent as compared with solid bodies.

Advantage is taken of the poor conducting power of air in many simple household contrivances. To keep a tea-pot warm it is surrounded by a "cosy". This is made of loosely packed woollen material. The wool is itself a bad conductor of heat, but its effect is greatly assisted by the air entangled between the threads of the wool. This is a worse conductor than the wool, and being prevented by the network of wool-threads from moving readily, cannot cause loss of heat by the method of transmission usual in gases, to be described later. The cotton-wool packing used in Experiments 57 and 58 has a similar function. The layer of snow which frequently covers the ground in severe winter weather is of great service as a means of preventing the underlying soil from losing so much heat that the plants growing in it suffer serious damage. The small crystals of ice, of which snow consists, are of low conducting power and the air between the crystals is again of very considerable assistance

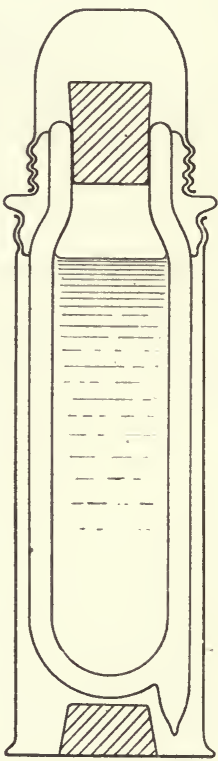

Fig. 57. in preserving plant life.

Empty space is a perfect non-conductor of heat. The "Thermos" flasks (see Fig. 57) which have come so much into use of late consist essentially of a doublewalled glass vessel, the space between the walls being rendered as completely vacuous as possible. Hot 
substances placed in the interior of the flask lose their heat very slowly, since conduction and convection (described below) are almost entirely prevented and the silvering of the glass further checks the loss which might occur by other means.

124. The general mode of propagation of heat in fluids is illustrated in the following experiments.

EXPERIMENT 68. Nearly fill a large beaker with water and place it on a piece of wire gauze on a tripod stand. Tear some blotting-paper into very small pieces and drop them into the water, allowing them to become completely soaked and fall to the bottom of the beaker. Heat the water by placing a small Bunsen flame under the edge of the beaker. The heated water will move upwards from the point of application of the heat and cooler water will take its place, the direction of motion of the water being indicated by that of the scraps of blotting-paper, provided that these are small enough.

Heat is here transmitted from one part of the liquid to another by actual motion of the heated portions of the water away from the source of heat, their place being taken by cooler water which becomes heated and moves away in turn. By continued repetition of this process the temperature is caused to rise in all parts of the liquid. Buildings are frequently heated by taking advantage of this motion of the hot portion of a liquid to a cooler region and the method em-

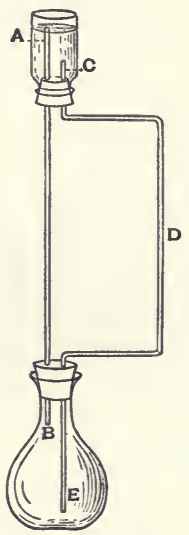

Fig. 58. ployed is roughly outlined in the next experiment.

Experiment 69. Fit up the apparatus of which Fig. 58 is an illustration. The upper vessel is a 
bell-jar, with a tightly-fitting rubber stopper, through which pass two glass tubes as shown. The flask below is likewise fitted and is completely filled with water. Pour enough water into the bell-jar to cover the end of the topmost tube. Drop a few crystals of potassium permanganate (or Condy's crystals will serve equally well) into the liquid in the jar. Fix the apparatus in an upright position by means of a clamp and retort stand, at such a height that a Bunsen flame may be placed underneath the flask. Light the gas and gently heat the flask with a small flame. The water will pass along the straight tube into the bell-jar and a descending current will flow down the bent tubing to the bottom of the flask. The course of the liquid may be traced by the pink colour of the solution of potassium permanganate. The circulation can be watched until the whole of the water has assumed a uniform tint.

The flask represents the boiler situated in the basement of a building, the vertical tube corresponds to the iron pipe leading from the top of the boiler to the top of the main hot cistern, the latter is represented by our bell-jar and the bent tube is comparable to the feed-pipes and radiators used to distribute the hot water to the various floors of the building. The smaller apparatus by which a supply of hot water for the bath-room and for general domestic use is maintained in most modern houses works on an exactly similar plan. Further details on this head will be found in Part II.

125. The currents set up in a liquid as a result of heating are called "convection currents", the heat being said to be transmitted by convection. This method of transmission does not take place with solids, 
the particles of which are not free to move over and around one another, as is the case with liquids. Gases resemble liquids in this respect, the motion of their particles being even less restricted than that of liquid particles. The setting up of convection currents in a gas by the application of heat is described in the next experiment.

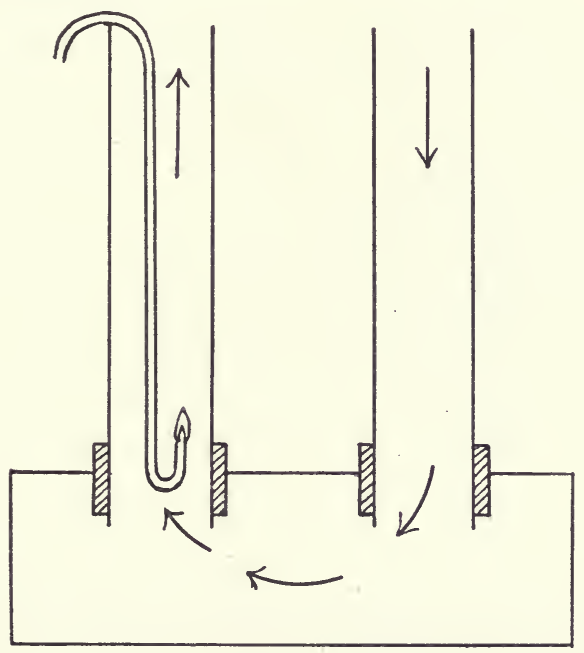

Fig. 59.

Experiment 70. The necessary apparatus consists of a flat cardboard or wooden box-a large cigar-box answers admirably-with all apertures such as the cracks around the edges of the lid carefully sealed by pasting paper over them. Two holes are made in the lid of sufficient size to admit the ends of wide glass tubes, such as Argand lamp chimneys, and the latter are kept in position as shown in Fig. 59 by bands of wide rubber tubing or by a plaster of Paris seal. 
A small gas-jet at the end of a bent glass tube attached to the ordinary gas supply, is lowered into one of the wide tubes as illustrated. An upward current of heated air ascends this tube and a current of cool air passes downward in the other. The presence of these streams of air may be rendered visible by holding a smouldering piece of soft brown wrapping paper at the top of the second tube, when the course taken by the smoke indicates the directions of the currents.

This experiment is an illustration on a small scale of a method used in ventilating coal-mines in which there is no danger of explosion on account of the presence of explosive mixtures of air with the gases sometimes given off in such mines. A large fire is built beneath the end of a deep shaft, known as the "upcast" shaft, and fresh air passes down a similar shaft, the "downcast" shaft, situated in the part of the mine furthest from the former. This air is thus obliged to traverse the various "workings" of the mine in its passage from one shaft to the other and the miners are enabled to continue at work without harm from the fouling of the air by the products of respiration. An ordinary room, in which a fire is burning, is ventilated in a similar fashion. The heated air goes up the chimney and fresh air enters through the apertures in the walls of the room, such as window and door openings and the cracks between the fittings of the woodwork, as well as through the porous brick and plaster of the walls themselves.

\section{Exercise for Student.}

Devise experiments whereby it may be shown that the cooling of one portion of a fluid sets up convection currents toward the cooled region. 
126. The chief method of propagation of heat through the substance of a fluid is then by means of convection currents while in solids transmission of heat is brought about by conduction. Thus another distinguishing feature as between fluid and solid bodies may be added to those already mentioned in Chapter v.

\section{Exercise for Student.}

Try to account for the inception of convection currents in such an experiment as No. 69 by the application of the knowledge already gained concerning expansion by heat, density, and fluid pressure.

127. When treating of the Kinetic Molecular Theory in Chapter v, it was stated that matter consisted of small particles, termed molecules, which were separated from one another by varying distances, according to the state of matter concerned. A question naturally arising from this conception of the constitution of matter is "What occupies the spaces between the molecules?" The view generally held at the present day is that a peculiar form of matter, of density exceedingly small when compared with that of any of the common forms, exists in these interspaces, and extends continuously even into those regions which we are accustomed to look upon as empty. This extremely tenuous substance is termed "ether.". (Care must be taken not to confuse this with the well-known liquid bearing the same name.) Ether fills all space and is a prime and omnipresent constituent of all portions of the universe. Our next subject for study will be the means by which heat is caused to pass through this universal medium, the ether of space. That it does so pass is a matter of common knowledge, since heat is received from the sun, a hot body situated over 90,000,000 miles from our Earth, and the sensible 
limits of our atmospheric envelope only extend outwards from the Earth's surface to a distance of at most some few hundred miles. Beyond this limit ether alone is thought to be the medium by means of which the vast gap is bridged, and the existence of the Sun made evident to our senses by the light and heat it affords.

128. Ether being continuous, no motion of its separate parts such as occurs in convection can be assumed to be the means by which heat is propagated through it. An entirely different form of motion, the up and down vibration we usually term wave-motion, is the method suggested. The light and heat emanating from the Sun set up this form of motion in the ether, the waves produced being exceedingly minute-so minute that many thousands of them are caused in so short a distance as one millimetre. These light and heat waves are simply ether in motion and the ether itself is neither illuminated nor heated by the passage of light and heat in this manner. When waves of a certain size strike an object, the sensitive membranes of the eye are affected and we see the object, by reason of the reflection of some of the waves towards the eye. Waves of a somewhat greater size do not excite the sensation of vision when they reach an object, but become evident to us by causing the object to become hot. We can now understand why the hand, placed beneath or at the side of a hot body, receives heat from the body, although, as previously stated, the heated air travels upward. The heat experienced by the hand situated underneath the hot object reaches it by setting up heat waves in the ether between the air molecules, and when these impinge upon the hand, the sensation of hotness is excited. That the 
air itself in this region is not heated by the passage of the wave-motion may be shown by interposing a screen, such as a sheet of paper, between the hand and the hot body. An immediate feeling of coolness is experienced, which could hardly be the case if the air itself were hot.

Experiment 71. Suspend the metal ball used in Experiment 40 in the middle of the flame produced by a powerful Bunsen burner. While the ball is being heated, hang two or three thermometers from the rings of retort stands so that the bulbs of the instruments are at the same level as the centre of the ball, and at different distances from the flame, say 10", 15", and $20^{\prime \prime}$. Each thermometer will record an increase of temperature, that nearest the flame showing the greatest change, and the most distant one the least. When the thermometer readings no longer increase turn out the burner and quickly remove the hot ball. Each will almost immediately register loss of heat as shown by the fall of mercury, denoting that the air in the neighbourhood of the instruments has not been made hot. This experiment may be varied by placing three thermometers at equal distances from the hot ball, after removal of the flame, one being placed $10^{\prime \prime}$ above the ball, a second $10^{\prime \prime}$ below it, and a third $10^{\prime \prime}$ away in the same horizontal plane.

\section{Exercise for Student.}

Account for any differences in the readings noted in the experiment just suggested.

An everyday application of the observations made in our experiment is to be found in the action of a person seated near a hot fire and seeking relief from H. D. S. 
the heat. The interposition of a book, the hand, or a fan, between the fire and the face is productive of an immediate sensation of coolness.

129. The name given to the method of transmission of heat we are now considering is "radiation", and we speak of the heat so transmitted as "radiant" heat. Rays of light and of heat are of similar character, some rays being able to give rise to both sensations. A familiar experiment will emphasise this point.

Expertment 72. Obtain a glass lens, such as is in common use for magnifying small objects. Hold this with one face turned towards the sun, and on the other side of the lens place a sheet of brown paper so that it lies in a plane parallel to that of the middle of the lens. A spot of light will be seen on the surface of the paper. This is an image of the sun, and, by moving the paper towards or away from the lens, this spot may be made to vary in size. Variation in brightness will accompany the change in size, the smaller the spot the brighter being its appearance. The lens is constructed in such a way as to bend the rays of light from their original paths, and to crowd them together at some spot near the lens. This is called "focusing" the rays. Heat rays are similarly bent in their passage through the lens and evidence of this may be afforded, if the lens be a good one, by the scorching of the paper when the spot of light is of least diameter. Should this not occur, place the hand or the builb of a thermometer so as to receive this smallest image of the sun, when the increased warmth at this spot will be readily shown.

130. All substances upon which heat rays strike are capable of receiving heat by this means but all do not exhibit this quality to the same extent. 
Experiment 73. Procure two small tinned iron vessels, such as the cans in which condensed milk, treacle, and such commodities are frequently packed for sale. Remove all paper from the outside of each. By holding one of the cans in the upper part of a candle or luminous gas flame, cover the bottom with a layer of soot, or as it is sometimes termed, lampblack.

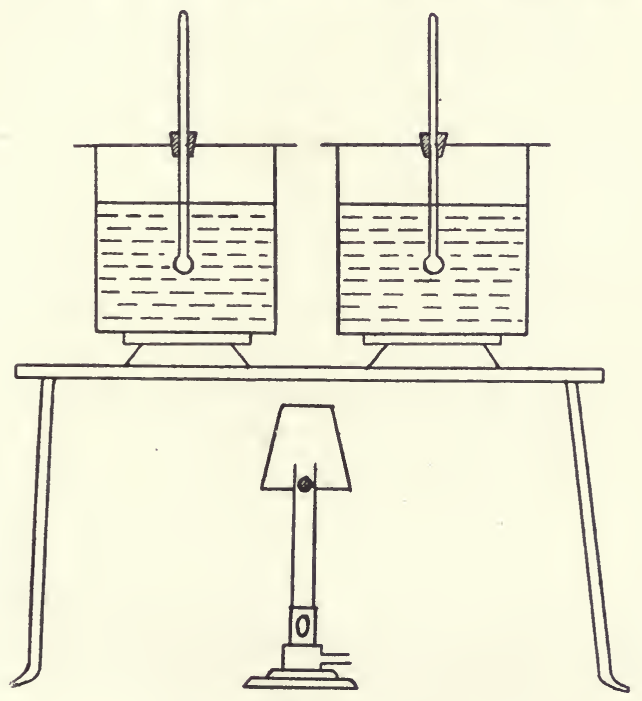

Fig. 60 .

Support the two vessels on the top of a large iron plate provided with iron supports, by putting pipeclay triangles under the cans as indicated in the figure. Arrange the triangles so that the distances from the cans to the plate are equal. Under the plate place a good Bunsen burner, making the distances of the bottoms of the cans from the burner as nearly equal as may be. To prevent uneven heating through draughts, the conical top shown in the diagram may 
be attached to the top of the burner. Half fill each can with water, and cover the top of each with a sheet of cardboard carrying a thermometer inserted through a cork in the centre of the sheet. See that the bulbs of the thermometers are submerged to equal depths in the water. Leave the apparatus at rest for two minutes and then read each thermometer. Light the burner, turning on both gas and air supplies to the fullest extent. Record the readings of the thermometers at intervals of about one minute, stirring the water with the thermometer before taking each reading. The rise of temperature registered by the instrument in the can with the blackened bottom will be more rapid than that recorded by the other. Continue the observations till the water in each can has shown a considerable gain in temperature. Remove the cans. from the hot plate and place them side by side in a quiet place, free from draughts. They should be kept out of contact with the surface of the bench or other supporting medium by placing the triangles underneath them as before. At intervals of a minute or two, read the thermometers, until the water has nearly reached the temperature of its surroundings. The water in the bright can will show the less rapid fall in temperature, counting the fall from the time at which the hotter water in the other reaches the temperature at which the water in the bright can was when the first reading during cooling was registered.

Bodies heated in the manner above described receive heat chiefly by radiation. The heat rays are more readily absorbed by the dull black surface and become sensible as heat. The bright surface, however, reflects a large proportion of the rays, just as a mirror reflects light rays, and a lesser proportion of the rays 
become sensible as heat, as was shown by the behaviour of the thermometer in the bright can. A common kitchen experience confirms our observations on this head. The water in a new kettle, when placed over a bright fire, takes a longer time to reach boiling-point than does water in the same kettle after repeated use has covered the bottom of the vessel with a layer of soot. Kettles for use on kitchen ranges should not be kept too scrupulously clean as regards the bottom of the utensil. On the other hand, the second part of our experiment suggests that the upper part of the kettle should be scoured if we desire that water which is being, or has been, heated in it, shall not lose the heat gained more rapidly than necessary.

131. The observations made above regarding the better absorption and radiation of heat by a dull and blackened surface than by a bright one are of general application. Dark-coloured, rough, or dull surfaces are in this respect contrasted with light, smooth, and bright ones. A rough test of this may be made by performing Experiment 71 again, placing two thermometers at equal distances from the source of heat, the bulb of one being covered with black paper and that of the other with white. Using instruments of similar construction, it will be found that the one with the black paper round it gains heat the more rapidly. Heat rays resemble light rays in being reflected from bright and smooth surfaces, hence bodies possessing either or both of these do not absorb heat readily.

Dark clothes are usually worn in cold weather in preference to lighter garments, and there is some show of reason in the choice. Any heat which reaches the clothes is absorbed to a large extent, but it is an open 
question whether the correspondingly greater radiating power does not almost entirely compensate for the advantage of ready absorption. The white flannel of the cricketer reflects heat better than would a darker material, but, on the other hand, the heat received from the body is not absorbed as readily. It is probably rather a matter of convenience which decides the colour of our clothes than the conscious application of experience, and dark materials find more favour in winter on account of the great labour which would be involved in keeping light garments clean in such a climate as ours during the cold season.

132. The chief points dealt with in this chapter may be briefly summarised as follows :

Solid bodies transmit heat by conduction alone, the heat received by the molecules nearest the source of heat being passed on to those next them, these latter passing on their heat in turn, and the continued repetition of this process resulting in the propagation of the heat in all directions through the solid.

Liquids and gases transmit heat chiefly by convection, actual motion of the heated part of the fluid taking place away from the heating agent to cooler regions and other parts of the fluid moving towards the neighbourhood of the source of heat, to be heated and move away in their turn.

Through space and also to a very large extent through gaseous media, heat is propagated by the setting up of wave motion of a certain magnitude, the medium being unchanged in temperature by the passage of the heat, and the latter only becoming sensible when the waves meet an object, when the energy transmitted by them is transformed into that form of energy which we call heat. 


\section{QUESTIONS}

1. Suggest a simple method of determining experimentally which of two spoons is made of silver and which of electro-plate.

2. Good radiators are good absorbers of heat. Describe an experiment in illustration of this statement.

3. Good reflectors of heat are bad absorbers. By what experiments would you try to show that this statement is correct?

4. Pieces of black and white cloth are spread out on the surface of snow in bright sunlight. The snow underneath the black cloth is observed to melt more quickly than that under the white. How do you account for this?

5. Why are blankets used to keep the body warm in winter and to prevent the melting of ice in summer ?

6. Pieces of wood or ivory are often inserted between the handle of a tea-pot and the pot itself. What is the reason of this ?

7. Why does the presence of a chimney assist a fire to burn more steadily and brightly than it does when no flue is provided ?

8. Why does the door-knob feel colder than the door, and the door colder than the carpet?

9. A Norwegian cooking-box consists of a large box thickly lined with straw or felt, provided with a lid similarly lined. Cookingvessels placed in the interior of the closed box remain warm for some hours. Give reasons for this.

10. A sheet of brown paper and a powerful magnifying glass are placed under the receiver of an air-pump in such positions that the paper is at the focus of the lens. The air is then exhausted from the receiver as completely as possible, and the whole apparatus placed in bright sunlight so that the lens faces the sun. Will the paper be burnt? Give arguments in support of your opinion 


\section{CHAPTER XII}

HEAT AND ENERGY. THE KINETIC THEORY

133. In a previous chapter we have briefly discussed the nature of heat and it was there stated that heat was a form of energy, i.e. that a given quantity of heat is able to perform a definite quantity of work. We already know that a weight of $10 \mathrm{lb}$., situated at a height of 10 feet from the ground, possesses 100 foot-pounds of potential energy. Further, we are aware that this potential energy may be transformed into kinetic energy if the weight be permitted to fall, and this kinetic energy may be made to do work by suitable means, e.g. the weight may be attached to a string passing over a pulley to the axle of which is fixed a small mill, in which coffee or other substance may be ground during the fall of the weight. A hot body resembles the weight in its elevated position. The hot body possesses energy and can perform work such as turning water into steam, the latter being caused to make a grindstone go round by some convenient mechanical contrivance. In thus effecting the performance of work, the hot body loses heat, and, unless a further supply is afforded, its temperature falls and at length reaches that of its surroundings, when its capacity for doing work is exhausted. It then resembles the weight resting on the ground, at the end of its fall, when the latter no longer possesses either potential or kinetic energy, or, in other words, has parted with its ability to do work. 
Just as potential energy may be transformed into kinetic energy, and heat energy, as mentioned above, may undergo a similar transformation, so may kinetic energy be changed into heat. A piece of lead, struck several times in rapid succession by a hammer, soon becomes appreciably hot. Very careful measurements of the quantity of heat that may thus be generated by the transformation of kinetic energy have been made by various investigators. The mean result of their determinations shows that, to raise the temperature of $1 \mathrm{lb}$. of water by $1^{\circ} \mathrm{F}$. requires the expenditure of about $775 \mathrm{ft}$.-lbs. of kinetic (or other form of) energy. Conversely, $1 \mathrm{lb}$. of water, in cooling through $1^{\circ} \mathrm{F}$., can perform $775 \mathrm{ft} .-1 \mathrm{bs}$. of work. Expressing this result in terms of the Centigrade scale, the number of ft.-lbs. becomes $775 \times \frac{9}{5}=1395$.

The magnitude of this quantity, which is termed the "Mechanical Equivalent" of heat, may be grasped if it be considered that the energy required to raise the temperature of 1 gallon of water from $15^{\circ}$ to the boiling-point is equivalent to that which would raise a weight of nearly $1 \frac{1}{2}$ tons from the pavement to the top of St Paul's Cathedral.

We have defined a calorie as the quantity of heat needed to raise the temperature of $1 \mathrm{~g}$. of water by $1^{\circ} \mathrm{C}$. Since 1 foot $=30^{\circ} 48 \mathrm{~cm}$., and $1 \mathrm{lb} .=453.6 \mathrm{~g}$., 1 calorie is equivalent to

$$
(1395 \times 30 \cdot 48 \times 453 \cdot 6) \div 453 \cdot 6=42,500
$$

gram-centimetres, i.e. to raise the temperature of $1 \mathrm{~g}$. of water by $1^{\circ} \mathrm{C}$. requires the expenditure of as much energy as would raise a weight of $425 \mathrm{~g}$. to a height of 1 metre.

134. We have already shown that hot bodies give up their heat to their surroundings, if these be at 
a lower temperature, and when heat has been distributed in this way, it is no longer available for the performance of work. All other forms of energy tend to be transformed finally into heat, and thus a continual disappearance of energy apparently takes place, the heat thus produced being readily dissipated. Some simple examples of this may be quoted. Coal possesses a store of energy by virtue of which it is able to unite with one of the gases of the atmosphere-oxygento form as the chief product another gas, called carbon dioxide. In so doing it produces much heat, for this union with oxygen is what we usually call the burning of the coal. The heat is soon shared with the air and other bodies in the neighbourhood of the burning coal, and the carbon dioxide is found to possess a much smaller store of chemical energy than the coal from which it was formed. Again, when an electrical current passes along a wire, part of the electrical energy is used up in raising the temperature of the wire. An example of this is to be found in the incandescent filament lamp. This heat also soon becomes unavailable for the execution of work. Another interesting example of the " degradation" of other forms of energy into heat is afforded by the recently discovered element, radium. This substance, whether free or combined, is found to be always slightly warmer-about $1 \cdot 5^{\circ} \mathrm{C}$.than its surroundings. This continuous production of heat energy on the part of the radium is accompanied by the formation of simpler and less active substances, the radium being said to be "degraded" during this process.

135. The next point to be considered is the form in which heat energy exists in hot bodies. We will first recapitulate briefly our previous statements concerning the Kinetic Molecular Hypothesis. According 
to this, substances are all composed of molecules, separated by varying distances, these latter being greatest for gases and least for solids. The molecules are in rapid motion, the freedom and rate of motion being again greatest in the case of gases, least in that of solids. The application of this hypothesis to the explanation of some of the various phenomena observed during the course of our study of heat follows.

136. Heated bodies were found to expand under the influence of the heat supplied. It is supposed that the molecules of a substance, when heated, are made to move with greater speed. As a result of this, they repel one another, during their frequent collisions, with greater force, thus exercising greater pressure upon their surroundings. If this increase of pressure be not counterbalanced in any way, the general effect of the combined increase of pressure in the part of the total number of molecules in any given body is to push away its surroundings to some extent, i.e. the body as a whole expands.

If the heated body be in the solid state, we have found that, as a general rule, it changes its state when a certain definite temperature is reached. Liquid molecules possess greater freedom of movement than those of solids, and in order to overcome partially the cohesion of the molecules of a solid substance, a large store of extra energy must be supplied to the solid, which then assumes the liquid state. This corresponds to our observation that a very large quantity of energy in the form of heat is used up in changing a solid into a liquid without rise of temperature. This energy, as already demonstrated, becomes again available in the form of heat when the liquid substance is caused to solidify once more. 
The marked difference in the freedom of movement of gaseous as compared with liquid molecules entails again the expenditure in general of an even larger quantity of heat than that demanded by the change of state from solid to liquid. The considerable change of volume occurring during vaporisation necessitates a further expenditure of energy in overcoming the external resistance of the surrounding atmosphere. The latent heat of vaporisation of a liquid is thus accounted for in overcoming the cohesion between the molecules of the liquid and in expanding the volume of the substance against external pressure.

137. Heat energy, then, resides in bodies as a mode of motion, although this motion is of too delicate a character to be detected as such by the most sensitive instruments at our disposal. Some colour is lent to this view, if we consider the question of what must occur during radiation. In this process motion of some kind is set up by a hot body, whereby heat is transmitted to another through the ether. Little consideration is needed to see that it would be against all experience for a body in a state of rest to set up a disturbance whereby this may be effected. Hence our idea that heat is a form of kinetic energy receives some support.

138. It may be well to expend a little further consideration on the subject of the action of heat upon gases. In Experiment 51 it was demonstrated that a gas, when heat was supplied to it, was able to overcome the pressure of the atmosphere to some extent. What would happen if, instead of expanding against a constant pressure, as is the case in the experiment quoted, the gas was prevented from expanding ?

Experiment 74. Pour mercury into the long limb 


\section{Heat and Energy. The Kinetic Theory 205}

of a Boyle's tube-as used in Experiment 35-and, by inclining the tube, cause some of the air from the closed limb to escape through the mercury. Immerse the lower part of the tube in a deep vessel containing water, so that the top of the closed limb is under water. Bring the tube to a vertical position, and mark the level of the liquid in each limb. This may readily be done by scratching the surface of the glass with a sharp file. Gently heat the vessel, and when the mercury begins to rise in the open limb, pour in more mercury in quantity sufficient to bring the level of that in the closed limb back to its original position. It will be found that, as long as the temperature continues to rise, constant supplies of fresh mercury will be required. From this it is evident that the gas, when prevented from expanding, exerts greater pressure as its temperature rises.

This increase of pressure is well shown if a bladder be inflated with air as fully as possible at the ordinary temperature and then be placed in front of a good fire. The increase of pressure brought about by this treatment is generally sufficient to burst the bladder.

When considered from the point of view of the Kinetic Theory, as set out earlier in the chapter, the increase of pressure noted is explained thus: The molecules move more rapidly as a result of the rise of temperature, and consequently impinge more frequently upon the walls of the containing vessel. It is the continual impact of the molecules which causes the gas to exert pressure, and when the frequency and force of impact of the striking molecule both receive augmentation as a result of heating the gas, the pressure of the gas shows a corresponding increment. 


\section{Exercise for Student.}

Assuming the Kinetic Molecular Hypothesis, try to account for the phenomena generalised in the statement known as Boyle's Law.

\section{QUESTIONS}

1. From what height must a weight of 1 ton be dropped if all the kinetic energy generated during its fall is to be enough to raise the temperature of $2 \mathrm{lb}$. of water from $32^{\circ} \mathrm{F}$. to $212^{\circ} \mathrm{F}$. ?

2. How much energy, expressed in kilogram-metres, is given out while $548 \mathrm{~g}$. of water cool from $65^{\circ}$ to $8^{\circ}$ ?

3. How much energy in the form of heat must be supplied to an iron cube with edges $6 \mathrm{~cm}$. long, in order to raise its temperature from $15^{\circ}$ to $150^{\circ}$ ? (Sp. heat of iron $=0.114$.)

4. Find the height to which a stone, weighing $500 \mathrm{~g}$., could be raised, if all the energy set free during the condensation of $10 \mathrm{~g}$. of steam could be expended in raising it.

5. The burning of $1 \mathrm{~g}$. of carbon produces 8080 calories. What weight of ice could be melted by the burning of $185 \mathrm{~g}$. of carbon ?

\section{CHAPTER XIII}

\section{AIR \\ Its Nature and Chief Constituents}

139. We have learnt in the preceding chapters that air is a gas and has both volume and weight. In this chapter the nature of air will be further investigated. To this end we will first perform the following simple experiment.

Experiment 75. Affix a short length of candle (the small candles used on Christmas trees are most suitable) to a wooden block by lighting the candle, 
allowing a few drops of the melted wax to drop on the block, and then holding the bottom of the candle on this melted wax until it begins to solidify. Over the candle place a tall glass lamp-chimney (see Fig. $61)$ so that the bottom of the chimney rests on the block and the candle is in the centre of the chimney. Watch the candle flame. It will be observed that it flickers and becomes smaller and smaller. If the

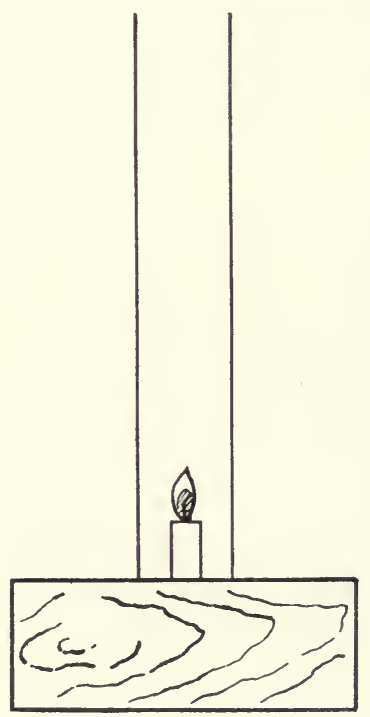

Fig. 61 .

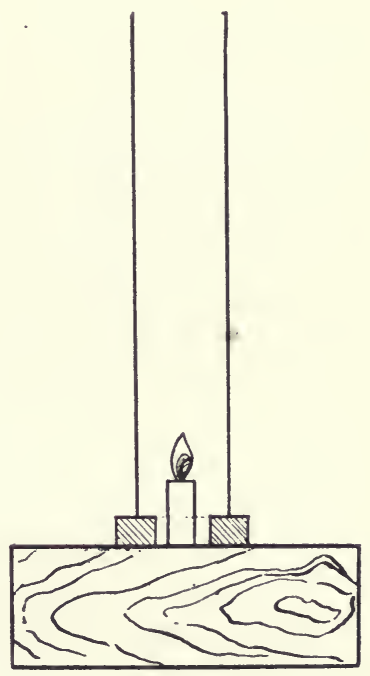

Fig. 62.

chimney be sufficiently tall and narrow, the candle at length goes out.

Relight it and replace the chimney, this time supporting the base of the chimney on two strips of wood (Fig. 62) so that there is an air-space between the base and the wooden block. Again note carefully the behaviour of the candle flame. It now burns 
more steadily and, if left undisturbed, will continue burning till the candle is entirely consumed. In the first case the candle had to burn in what was practically a limited supply of air, for very little fresh air is able to force its way down the lamp-chimney against the stream of hot air and products of combustion proceeding from the flame.

In the second case, fresh supplies of air passed in through the air-space beneath the chimney, and thus a continuous current passed up the chimney. To show the existence of this air-current let us try

Experiment 76. Arrange the candle and chimney as in Fig. 62. Light a wax-taper, and when well alight, blow it out. Place the smoking end near the air-space between the chimney and block. Note the direction taken by the smoke.

(This should be done in a room free from draughts, or the observation made may not be very definite.)

140. From the above experiments it is clear that air is necessary for the burning of a candle to take place. Other combustible bodies such as coal, wood, coal-gas, brimstone, and paraffin oil, also require a good supply of fresh air in order that their burning may be continuous and complete.

While dealing with this matter, we may note that the two simple experiments just performed illustrate the value of a fire in an open fireplace as a ventilating agent. The fire corresponds to our candle, the housechimney to our lamp-chimney, and the openings in the walls of the room (e.g. the opened part of the window, if any, and the cracks beneath and around the doors and the window-sashes) to the air-space provided between lamp-chimney and block. Fresh air is continually entering through these openings while the fire 
is burning, and this helps to keep the air of the room fit for respiration. (Cf. Experiment 70.)

141. We cannot well show directly that fresh air is necessary to the continuance of animal life. We must rest content with the statement of the fact that some of the operations which go on inside our bodies are somewhat similar in character to those involved in the burning of a candle, and require just as urgently for their due performance a good supply of fresh air. Lessening this supply lowers the vitality of the body just as under similar circumstances the candle flame flickers and weakens.

142. Having discovered that air appears to be necessary for burning to occur, let us next try to find out whether all the air takes an active part in the burning.

Experiment 77. Fix a short piece of candle, $C$, to a length of stout copper wire, $W$, as in Fig. 63. Push the end of the wire through a sheet of stiff cardboard, $M$, and light the candle. Lower the card and wire into a deep glass jar, $G$, so that the card rests on and closes the mouth of the jar. In a short time the candle flame will be extinguished.

Remove the candle, relight it, and quickly replace it in the jar. This time it goes out at once, or, if the removal and replacement have not been done deftly enough, burns for a very short time, much shorter than when previously plunged into the jar.

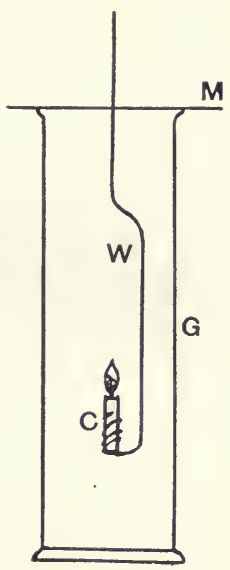

Fig. 63.

It is evident from this experiment that the air in H. D. S. 
the gas-jar, $G$, is no longer capable of supporting the burning of a candle. We do not yet know, however, whether it can support the burning of other bodies. We will therefore try to burn something else in the jar. Phosphorus is a suitable substance.

It is so highly inflammable that it is kept in bottles filled up with water, and must be handled with the greatest care.

Experiment 78. From a stick of phosphorus, cut off a small piece about the size of a pea, the phosphorus being held under water in a dish. Remove the phosphorus from the water with a pair of crucible tongs, place it on several sheets of blotting- (or filter-) paper, and press it gently between the sheets, carefully avoiding contact between the fingers and the phosphorus. When the phosphorus has been quite dried in this manner, transfer it by means of dry tongs to a small spoon, called a deflagrating-spoon, similar to that shown at $S$ in Fig. 64. The spoon should have a long wire handle as shown and this may either be pushed through a piece of cardboard like that used in Experiment 77, or, as is more usually done, pushed through a piece of cork, $K$, contained in a small cup on the upper side of the brass $\operatorname{cap} C$. Ignite the phosphorus by holding the spoon for a moment in the flame of a Bunsen burner. Remove the candle from the jar used in Experiment 77, and at once lower the burning phosphorus into

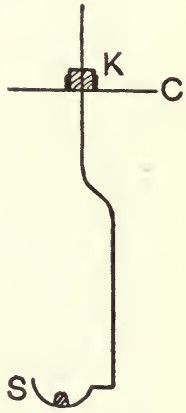

Fig. 64 . the jar till the brass cap rests on the mouth of the jar. (Be careful to arrange beforehand the length of wire between spoon and cap so that the former does not 
quite touch the bottom of the jar.) It will be found that the phosphorus continues burning for some time.

Thus, although the air left in the jar will not keep a candle burning, a piece of phosphorus still. remains alight. Evidently phosphorus is a more vigorous combustible than are the constituents of an ordinary candle.

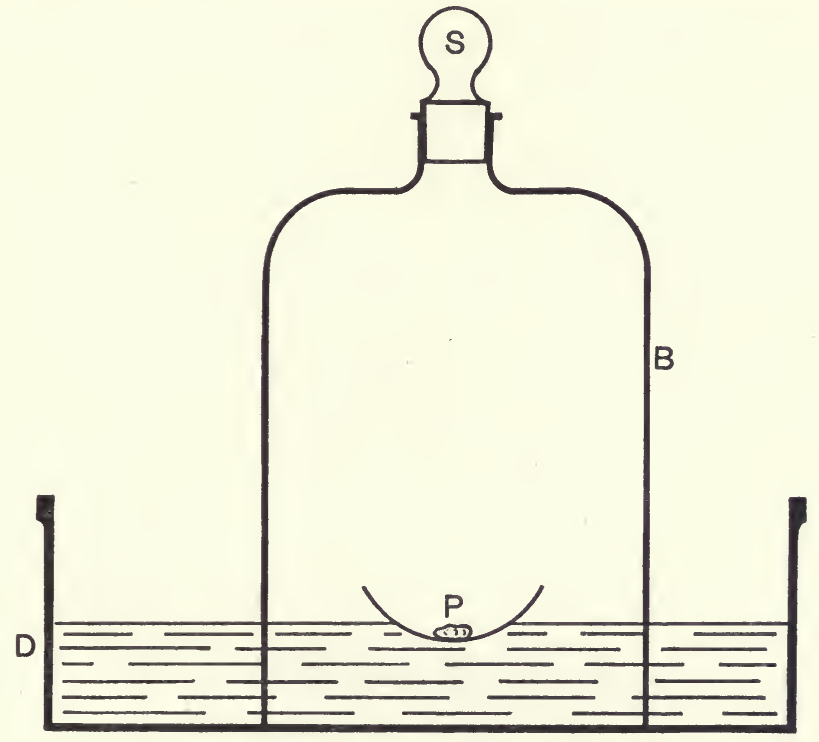

Fig. 65 .

Phosphorus, in fact, burns more vigorously in air than any other substance with which we are acquainted, and it is for that reason that we use it in our next experiment, in which we endeavour to find out whether all the air takes part in the burning.

143. Experiment 79. Fill the circular earthenware dish, $D$ (Fig. 65), half full of water. Carefully 
dry a piece of yellow phosphorus as big as a large pea and place it in a clean dry porcelain dish, $P$.

Float this dish on the surface of the water in the trough. Remove the stopper from a glass bell-jar, $B$, about 9 inches high, and lower the open end of the jar into the water so that the dish is within the jar. Note the level of the water-surface.

Warm a glass rod gently in the Bunsen flame and, passing it through the neck of the bell-jar, ignite the phosphorus by touching it with the rod. At once withdraw the rod and insert the glass stopper, $S$. Hold the jar down for a few moments, as the action is sometimes rather vigorous. Carefully observe how the phosphorus burns, what sort of product is formed by its combustion, what happens to that product, and also any alterations which occur in the level of the water-surface. When no further change seems to be taking place within the jar, note the fraction of the original air-space which is now occupied by water, and the appearance of the gas remaining in the jar above the water. Pour water into the trough till the waterlevel is the same both outside and inside the jar.

Warm the glass rod again, remove the stopper, and try to ignite any phosphorus which remains. If the experiment has been properly carried out this cannot be done. Insert a lighted taper through the neck of the bell-jar. It is at once extinguished.

\section{Exercise for Student.}

Why is the pouring in of water to equalise the levels necessary ?

From the above experiment it is clear that the whole of the air does not take part in the burning of a candle or a piece of phosphorus. 
144. It is probable that, from the above experiment, a rough idea of the volume of gas which remains, and does not assist in the burning, may be obtained.

To measure this volume more accurately, the following experiments may be performed :

EXPERIMENT 80. Down the side of a tall gas-jar paste a long strip of paper divided into centimetres and half-centimetres, the centimetre divisions being numbered in order, commencing from the closed end of the jar. Cover this paper strip with a layer of paraffin wax by means of a camel-hair brush dipped in some melted wax.

Half fill an earthenware trough with water. With a blunt knife blade, carefully scrape clean a small stick of yellow phosphorus about half an inch long, the phosphorus being held under water while scraping it. Coil a length of rather stout copper wire, as shown in Fig. 66, so that the diameter of the larger coils at the top is slightly greater than that of the phosphorus. To the lower end of the wire attach a flattened leaden bullet (or other small flat heavy substance) of sufficient weight to keep the wire upright when the flat surface is resting on the table. Put the freshly scraped stick of phosphorus into the coil (see Fig. 66). Invert the gas-jar previously prepared, and push the phos-

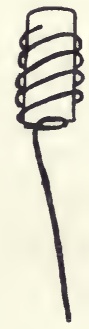

Fig. 66. phorus up into it by means of the wire, the length of which should be such that the phosphorus reaches the middle of the jar. Lower jar and contained wire into the water in the earthenware trough, so that they occupy the position shown in Fig. 67 .

Record the length of the column of air within the 
cylinder by noting the reading at the water-level inside the jar. Allow the apparatus to stand for a day and again determine the length of the air-column. A further reading after another interval of five or six hours may be made, when it will probably be found to agree fairly closely with the one last obtained. To ascertain the nature of the gas still contained in the gas-jar remove the bullet and any remaining phosphorus by raising the jar and carefully withdrawing

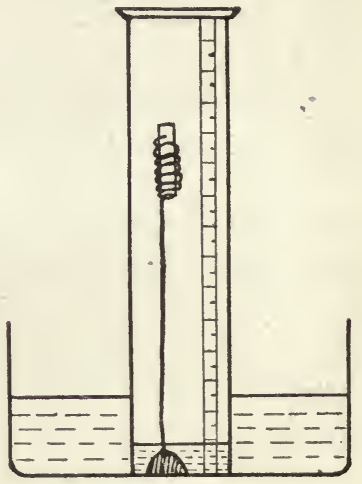

Fig. 67. them, keeping the mouth of the jar under water. Slip a glass plate under the mouth of thejar without removing the mouth from the water, press the plate firmly on the jar, remove from the trough and invert. Test the gas left in the jar by inserting a lighted taper and a piece of burning phosphorus (as in Experiment 78). It will be found that it does not support combustion.

If we assume, as may be usually done without serious error, that the area of cross section of the cylinder is the same throughout its length, then the volumes of gas enclosed in the cylinder are directly proportional to the lengths of the portions of the cylinder occupied by them.

Our first reading, then, is a measure of the volume of the air in the cylinder, and our second of that part of the air which does not support combustion. By subtracting the latter from the total volume of air, we obtain a measure of the volume of the part which disappears during combustion. It will be found that the 
portion remaining unconsumed bears to the latter a ratio of about 4 to 1 .

\section{Exercise for Student.}

Detect other slight sources of possible error in the relative volumes of air and of nitrogen thus obtained.

145. The part of the air which supports burning is called oxygen, while the other portion is termed nitrogen $^{1}$, and we have learnt that air is made up of about 4 volumes of nitrogen with 1 volume of oxygen. A second determination of this volume-ratio may be made by the method of

Experiment 81. Put about an ounce of clean iron filings on a piece of muslin, and make the latter into a bag by bringing the corners together and tying them with thread. Attach this bag of iron filings to one end of a length of stout copper wire, dip the bag in water and arrange the apparatus shown in Fig. 68, where $M$ is the bag, $J$ a graduated gas-jar similar to that used in the last experiment, and $T$ an earthenware trough.

Read off the volume of enclosed air.

Allow the apparatus to stand for a week. Again read off the volume of the enclosed gas, and test its nature as in Experiment 80.

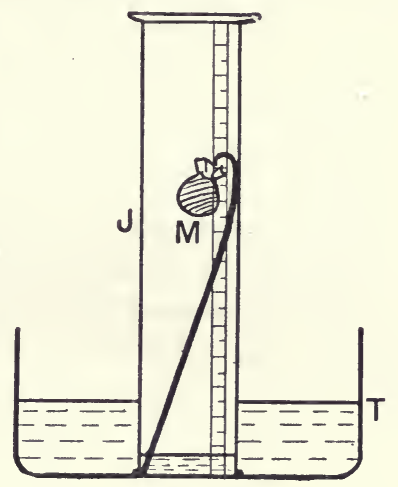

Fig. 68.

Then remove the bag and examine the appearance of the iron filings, comparing it with that before they were inserted in the jar. 
The ratio $\frac{\text { volume of nitrogen }}{\text { volume of oxygen }}$ as determined from the readings should agree fairly closely with that obtained in the previous experiment.

146. In Experiments 80 and 81 the action of the phosphorus and iron on the air resembles that of the burning phosphorus, although in these experiments no production of either heat or light will have been noticed. In all three cases, what has occurred is that the phosphorus (or iron) has united with a part of the air, namely, the oxygen, and formed by combination with this gas a new solid substance, which occupies a very small volume compared with that of the gaseous oxygen absorbed in its formation. Thus the white powder formed when the phosphorus is burnt is called " oxide of phosphorus", and the brown powder (which we usually call rust), produced by the action of moist air on the iron, is termed "oxide of iron". In Experiments 79 and 80 the white oxide of phosphorus slowly disappeared because it dissolved in the water present, and in order to see more clearly the nature of this substance we will perform

Experiment 82. Carefully clean and dry a bell-jar, $B$, and a large glazed tile (or plate), $T$. Dry a very small piece of phosphorus between filter-papers, and place it on the middle of the tile (Fig. 69). Ignite it with a warmed rod or wire and im-

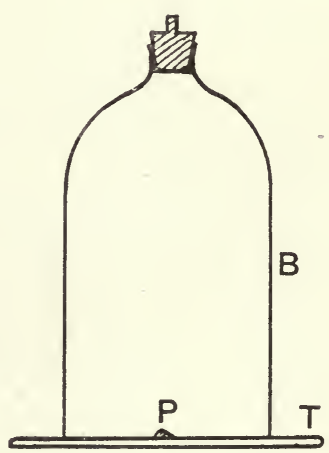

Fig. 69 . mediately place the bell-jar over it. 
When the phosphorus has ceased burning, allow the white fumes to settle, and then raise the bell-jar and examine the substance left on the tile. This is the oxide of phosphorus. Should the phosphorus begin to burn again when the jar has been removed, a further quantity of the oxide may be obtained by replacing the jar, and waiting till the phosphorus again stops burning.

(Note what happens to the oxide if it is allowed to remain in the open air for a few minutes.)

147. Such changes as those considered in this chapter are termed "chemical" changes. The union of two distinct substances to form a third, entirely different in properties from either, is often briefly expressed by such statements-termed " chemical equations "-as the following:

(1) Phosphorus + oxygen $=$ phosphorus pentoxide.

(2) Iron + oxygen $=$ iron oxide.

Here the sign "+" means "combines with" and "= " means " to form".

Thus statement (1) should be read "Phosphorus combines with oxygen to form phosphorus pentoxide". Phosphorus pentoxide is the exact chemical name of the substance we have hitherto called "oxide of phosphorus", and is required because we know of other distinct compounds of phosphorus and oxygen. Similarly iron rust, which we have called simply "iron oxide", chiefly consists of one of the three oxides of iron, called "ferric oxide" (Latin, ferrum, iron), combined with a certain amount of water.

148. We will next consider the action of air on the metals magnesium and copper. If magnesium, a metal with a bright silvery lustre, be allowed to remain in contact with moist air, the surface of the metal becomes somewhat dull, but no other change of importance can 
be noted. When the magnesium is strongly heated in air, however, a striking change takes place.

Experiment 83. From a coil of magnesium tape, cut off a piece about 2 inches long. Holding this with a pair of crucible tongs, place one end of it in the flame of a match or a Bunsen burner. After a short interval the metal ignites. Remove it from the flame, and note carefully the nature of the light obtained, and of the product left after the burning is over.

In this case we have a chemical combination taking place. The magnesium combines with the oxygen of the air to form magnesium oxide.

Magnesium + oxygen = Magnesium oxide.

When the combination has once been started it proceeds rapidly with the evolution of great heat and a most vivid light. (Compare the behaviour of phosphorus with that of magnesium in this respect.) This production of heat, and frequently of light as well, is a general accompaniment of a combination between two substances to form a third. Even when iron rusts in air, heat is produced, but the rate at which it is produced is so slow that we cannot detect it by ordinary means.

It is interesting to note that by special arrangement of the experiment, magnesium may be made to combine with nitrogen, forming magnesium nitride. This experiment, however, is too difficult for us to attempt. By taking advantage of this action, the gas argon was isolated in 1894 by Sir William Ramsay, from nitrogen obtained from the atmosphere.

EXPERIMENT 84. Place a short coil of bright copper wire in a hard glass tube closed at one end, a rubber stopper, through which passes a short length of glass tubing fitted with a tap, being fixed firmly in the open 
end. (See Fig. 70.) Open the tap and connect the glass tube by means of stout rubber tubing to an airpump or a good water-pump. Exhaust the air from the hard glass tube as completely as the efficiency of the pump permits. Close the tap, and disconnect the rubber tubing. Fix the hard glass tube in a clamp attached to a retort stand and strongly heat the tube and its contents with a powerful Bunsen flame for about five minutes. Remove the flame and note the appearance of the copper wire as a result of this treatment. If the evacuation of the tube has been thoroughly carried out the wire should still be bright.

Open the tap, thus causing the tube to be again filled with air. Again heat the tube strongly and watch the change in the appearance of the copper. After a

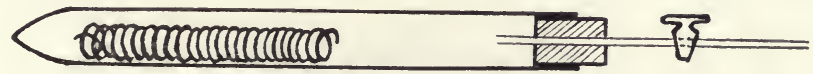

Fig. 70 .

short time it will entirely lose its brightness and the exterior of the wire will be covered by a black film. This is copper oxide, formed by the combination of the copper with oxygen from the air.

This experiment emphasises the important part played by the air in many of the chemical changes which take place under ordinary conditions. The absence of the active constituent of the air-oxygen-is sufficient entirely to prevent chemical change in the copper as a result of heating it.

EXPERIMENT 85. Hold a length of bright copper wire, by means of the crucible tongs, in the flame of a Bunsen burner till it is red-hot. Remove it from the flame, and note the behaviour of the copper while cooling and the appearance of its surface when cold. 
It will be evident that copper does not unite with oxygen so readily as magnesium, but the blackish appearance of its surface is nevertheless due to the production of a thin film of copper oxide.

149. Experiment 86. Fit up carefully the apparatus shown in Fig. 71, leaving the jar, $J$, however, inverted and filled with water, on the bottom of the trough. Weigh the roll of copper gauze, before insertion in the tube, on a balance which weighs correctly to 1 centigram.

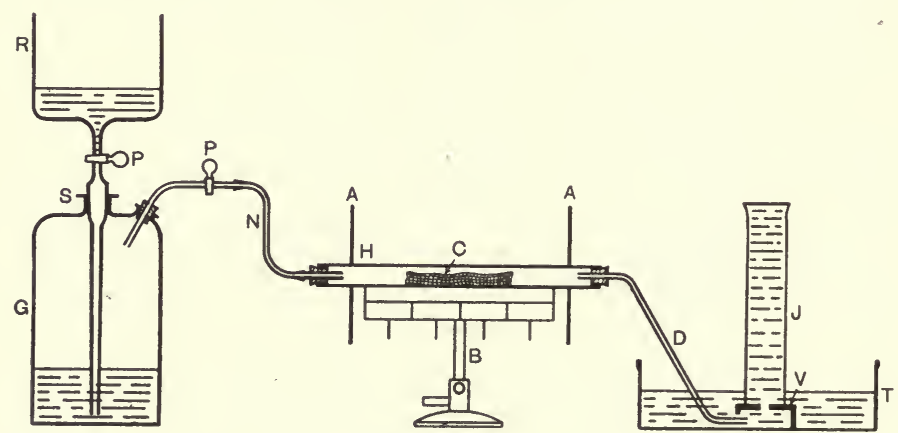

Fig. 71 .

$G \quad=$ gas-holder filled with air.

$R \quad=$ reservoir of gas-holder.

$P, P=$ well-greased glass stopcocks.

$D=$ glass delivery tube.

$A, A=$ square shields of asbestos millboard

$H=$ piece of hard combustion tubing about $\mathrm{l} \mathrm{ft}$. long and $\frac{3}{4}$ inch in diameter.

$C=$ roll of bright copper gauze about 5 in. long.

$B$ = Ramsay burner (a row of Bunsen burners may be substituted for this).

$N=$ stout rubber connection.

$J \quad=$ large gas-jar.

$T=$ earthenware pneumatic trough.

$V \quad=$ beehive cell.

$S=$ tight-fitting hollow glass stopper. 
Pour water into the reservoir, $R$, keeping the taps $P, P$, closed. Light the burner, $B$, using only a small flame at first. Gradually increase the gas supply until the gauze is at red heat, and no more bubbles escape from the end of the delivery tube. Place the jar $J$ in position on the beehive cell, and turn the stopcocks $P, P$, so that a slow stream of air passes along the tube over the heated copper. The rate at which the air passes must not be so great that the bubbles rising in the jar cannot be easily counted.

When the jar is full of gas, turn off the taps, discontinue the heating, and remove the end of the delivery tube from the water.

After the tube has become cold, remove the copper gauze and weigh it carefully. Test the gas collected in the jar with respect to its power of supporting the burning of a taper.

From this experiment we learn that copper, when strongly heated, burns, i.e. combines with the oxygen of the air to form copper oxide, but does not combine with the nitrogen. The combination with oxygen is also attended by increase of weight. This experiment may be repeated using a layer of "reduced" iron in the place of the roll of copper gauze. In this case it is better to weigh the tube and iron together before heating, and to weigh tube together with iron oxide after it has cooled. Had we carefully collected and weighed the products of oxidation (combination with oxygen) of a candle, phosphorus, magnesium, and iron filings we should have found that in each case these products weighed more than the original candle, phosphorus, magnesium, or iron respectively. This increase in weight is in every case the weight of the oxygen which has entered into combination. 
150. By carrying out experiments similar to the last experiment very carefully, the percentage weights of oxygen and nitrogen present in air have been found to be

$\begin{array}{llll}\text { Nitrogen } & \ldots & \ldots & 77 \text { per cent. } \\ \text { Oxygen } & \ldots & \ldots & 23 \text { per cent. }\end{array}$

As a result of many careful experiments on the percentage composition of air by volume, the following numbers have been obtained :

$\begin{array}{llll}\text { Nitrogen } & \ldots & \ldots & 79.04 \text { per cent. } \\ \text { Oxygen } & \ldots & \ldots & 20.96 \text { per cent. }\end{array}$

(Argon is here included in each case with the nitrogen, and certain other constituents of ordinary air, which are dealt with in Chapter XIV, are removed before the analyses are made.)

\section{QUESTIONS}

1. What do you understand by the term "oxide" ? Name three oxides, and give directions for the preparation of a specimen of each.

2. Describe fully how you would show that air consists of at least two portions, one of which takes part in the burning of a match, while the other does not.

3. A quantity of black powder is placed in a porcelain crucible, and both crucible and contents are carefully weighed. They are then heated to a bright red heat in a furnace for ten minutes. On cooling, the powder is found to be of a dark brown colour, and on weighing crucible and powder, their combined weight is now greater than before. What is the probable explanation of these changes ?

4. A piece of phosphorus is burnt in a closed vessel of air standing over water. The volume of the vessel is 1245 c.c. What volume of gas is left after the phosphorus has ceased burning and the apparatus has cooled? 
5. Suggest reasons for

(a) The extinction of a candle after it has burnt for a short time in a closed gas-jar.

(b) Phosphorus burning readily in air which will no longer support the combustion of a candle. .

6. Why does opening a window sometimes cure a smoky fire?

\section{CHAPTER XIV}

\section{MINOR CONSTITUENTS OF ALR}

151. In the last chapter, the two chief constituents of air, oxygen and nitrogen, were dealt with, and these two make up together about $97 \cdot 6$ per cent. by volume of our atmosphere. Argon, the gas formerly measured with the nitrogen, amounts to about 0.9 per cent., leaving 1.5 per cent. to be accounted for. The bulk of this remainder consists of two substances, and our next business is to find out what they are.

Expertment 87. Burn a candle in a dry clean gasjar as in Experiment 77. Notice that during the burning the sides of the jar become moist. This moisture, if sufficient be obtained, may be shown by suitable tests ( $v$. Chapter XVII) to be water. When the candle has gone out, remove it, and pour a little clear lime-water into the jar. Cover the mouth of the jar with a glass plate, and shake the jar vigorously. The clear lime-water becomes turbid, and if allowed to stand, a fine white powder settles on the bottom of the jar. Shake up some lime-water in a jar of ordinary air. No perceptible turbidity will be produced. We have thus obtained evidence of a second product of combustion of the candle. It is an invisible gas, like air, 
since it cannot be seen in the jar, but differs from air in that it is able to render lime-water turbid. This gas is called carbon dioxide.

152. Before considering the explanation of this formation of water and carbon dioxide, we will define the exact meaning of the two names "element" and " compound" as used by the chemist.

An Element is a substance which has not yet been split up into, nor built up from, simpler substances. The two gases oxygen and nitrogen, the liquid mercury, and the solids phosphorus, magnesium, copper, and iron, are elements. In all about 80 bodies are known which are believed to be elementary in character.

A Compound is a substance produced by the chemical union of two or more elements. It usually differs widely in appearance and properties from any of the elements of which it is composed.

Thus water, a liquid which extinguishes flame and does not itself burn, is a compound of two gases, one of which, hydrogen, burns readily, while the other, oxygen, causes bodies which are already burning in the air to burn still more brightly when they are placed in it. Again, phosphorus pentoxide is a white powder which we have shown to be extremely soluble in water. It is a compound of phosphorus, a yellow solid which will not dissolve in water, and the colourless gas, oxygen, which is only very slightly soluble in that liquid.

153. A candle is almost entirely composed of compounds of the two elements carbon and hydrogen (see Chapter xvII). When these bodies burn in air they are oxidised, i.e. combine with oxygen, and the reactions which occur may be briefly stated in the form of chemical equations as follows : 
Carbon + oxygen $=$ carbon dioxide.

Hydrogen + oxygen = hydrogen oxide.

("Hydrogen oxide" is the chemical name of water.)

We cannot see that these bodies are formed because the carbon dioxide is a colourless gas, while the water formed at the high temperature of the flame exists as steam and as such passes away from the neighbourhood of the flame as a colourless vapour. If this vapour meets a cold surface, such as the inside of the gas-jar in Experiment 87, it is condensed, and forms small drops of water. In this way the existence of the water formed by the burning of the candle is rendered evident to our sense of sight.

In showing by means of the lime-water test that carbon dioxide is also produced we depend on the fact. that the lime in the lime-water combines with carbon dioxide. Thus :

Calcium oxide + carbon dioxide $=$ calcium carbonate
(Lime)
(Chalk)

Calcium carbonate is the chemical name of chalk (the chalk of our cliffs and chalk-pits, not the prepared substance used for blackboard writing) and, as chalk will not dissolve in water, it separates out as fast as it is produced, forming the white substance which renders the lime-water turbid and settles on the bottom of the jar. When a solid substance separates out from a clear liquid in this way, it is said to be "precipitated", and our white sediment is called a "precipitate" of chalk.

It must be clearly understood that, as shown in Experiment 78, all the oxygen contained in the air in the jar does not combine with the constituents of the candle. A part only is thus used up, and a still smaller

H. D. S. 
volume of carbon dioxide is produced, some of the oxygen combining during burning with the hydrogen of the candle. Such a statement as "the oxygen in the jar is replaced by carbon dioxide during the burning of the candle" is consequently incorrect in that no mention is made of the partial character of this replacement.

154. All our common fuels-e.g. wood, coal, coalgas, paraffin oil-consist chiefly of carbon and hydrogen compounds, and, when burnt, produce water-vapour and carbon dioxide.

Experiment 88. Hold a clean dry glass bell-jar (or large beaker) for a few seconds over each of the following in turn :

(a) A burning splinter of wood.

(b) A small gas-flame.

(c) The flame of a paraffin lamp.

(d) The flame of a spirit lamp.

Note in each case the appearance of the inside of the jar, and after each test, close the mouth of the jar with a large glass plate, invert, and shake up the contents of the jar with a little clear lime-water.

It was stated in Chapter XIII that some of the operations which go on inside our bodies resemble burning. We are now prepared to show that this resemblance is very close.

Experiment 89. Breathe into the open end of a large dry cold test-tube. Note the appearance of the inside of the tube after the breathing. Now pour a little clear lime-water into the test-tube, and shake vigorously, observing carefully any alteration in the appearance of the liquid.

From this experiment it will be clear that watervapour and carbon dioxide are both to be found in 
expired air. They have already been shown to be the products of combustion of substances containing carbon and hydrogen. Now bread, meat, vegetables, milk, cheese, and all other food-stuffs contain carbon and hydrogen, and by their slow oxidation during the vital processes two of the chief bodies produced are water and carbon dioxide. (For a method of showing the presence of carbon and hydrogen in food-stuffs, see Part II.)

155. In the preceding chapter it was shown that chemical combination is usually attended with the evolution of heat. That heat is given out during the oxidation of the constituents of our food is rendered evident by the fact that our bodies maintain an almost even temperature, although our surroundings are usually at a temperature considerably below that of the body. The heat given out by the oxidation is used to maintain this difference of temperature between our bodies and their surroundings.

156. It was stated in paragraph 149 that the products of the burning of a candle weighed more than the candle itself. To test this, the following experiment may be performed.

Experiment 90. Arrange the apparatus shown in Fig. 72 .

Before beginning the experiment, weigh the candle, chimney, and $U$-tube with their connections and corks. Attach this weighed portion of the apparatus to the aspirator, and turn the stopcock so that a fairly rapid stream of water issues. This will cause a steady current of air to pass up the chimney and through the $\mathrm{U}$-tube into the aspirator. Remove the candle, light it, and quickly replace cork and candle in position as in the diagram. Arrange the rate at which the current 
of air is drawn through the apparatus, by regulating the outflow of water from the stopcock, so that the

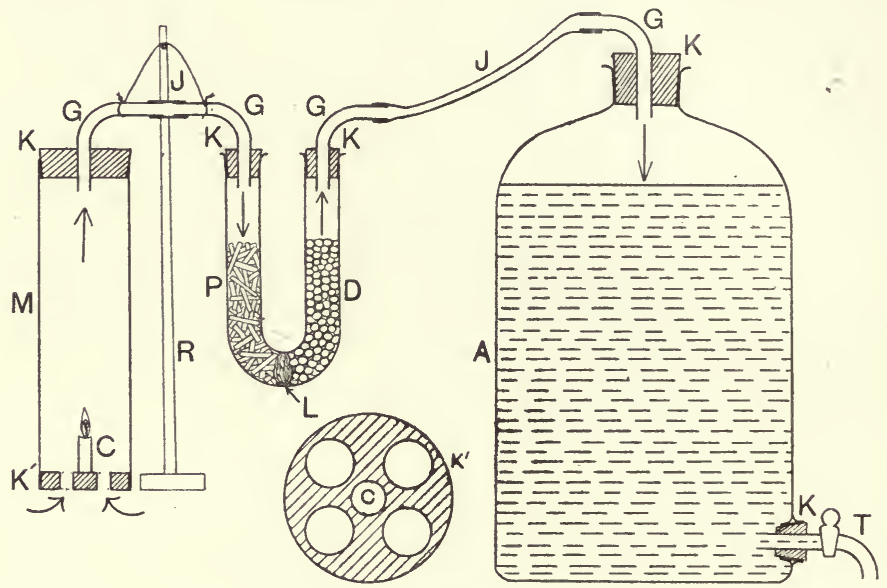

Fig. 72.

$K, K, K, K, K=$ tight-fitting corks.

$K^{\prime}=$ cork on which candle is fixed, cut as shown in small plan below diagram.

$C=$ short piece of candle.

$G, G, G, G=$ glass connections.

$J, J=$ rubber connections.

$T=$ glass stopcock.

$P D=$ glass $\mathrm{U}$-tube containing short sticks of caustic potash in the limb $P$, and small lumps of calcium chloride in $D$.

$L=$ loose-fitting plug of glass-wool.

$M=$ lamp-chimney.

$R=$ retort stand.

(Note. Greater efficiency results if the aspirator is replaced by a good water-pump, should this be available.)

candle burns freely and, as far as possible, without smoking. The products of combustion, being gaseous, 
are carried forward with the air-current, and absorbed in the U-tube.

The caustic potash retains the carbon dioxide by combining with it, resembling lime-water in this respect. It is, however, a much better absorbent than lime-water. Thus :

Potassium hydroxide + carbon dioxide

(Caustic potash)

$=$ potassium carbonate + hydrogen oxide. (Carbonate of potash) (Water)

The potassium carbonate, being a solid, remains in the tube, while the water simultaneously produced, together with that formed by the burning of the candle, combines with the potash or with the calcium chloride in the other limb of the $U$-tube, forming a solution which remains on the exterior of the small lumps.

After about one-quarter of an inch of candle has burnt away, blow out the candle, and, after a few seconds, turn off the tap. When the apparatus has cooled to the temperature of the room disconnect the aspirator, and again weigh the portion weighed before.

It will be found that, although some of the candle has disappeared, and has been, in everyday speech, "destroyed", the weight has increased. This increase in weight is due to the oxygen which has entered into combination with the constituents of the candle. While it exists as free oxygen in the atmosphere, it affects both pans of the balance equally, but when combined with carbon and hydrogen, and absorbed by the potash and calcium chloride, its weight is readily obtained.

157. The question next arises " Since the products of combustion weigh more than the original substance, 
does any creation of new matter take place?" To answer this question, the balance is again brought into use, but this time the whole of the apparatus is weighed.

Experiment 91. Cut off under water a small piece of yellow phosphorus. Remove it from the water, and dry it carefully with filter-paper. Drop it gently into a stout round-bottomed flask, or bolthead, $F$, of from 1.5 to 2 litres capacity. Push in a tight-fitting rubber stopper, $R$, and wire the stopper in firmly with copper wire. Weigh the flask and its contents carefully.

Holding the neck with a duster, bring the part of the flask where the phosphorus is, into a small Bunsen flame. The flask should be held at arm's length with the neck pointing toward the experimenter. As soon as the

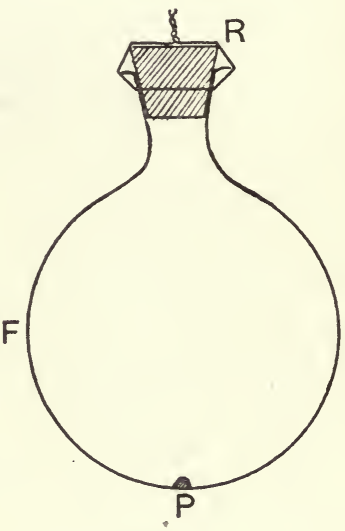

Fig. 73. phosphorus ignites, withdraw the flask from the flame, and hold it steadily away from the body till the phosphorus ceases burning. Allow the flask to cool down, and when it has regained the temperature of the room, weigh it carefully. It will be found that its weight is unaltered.

(Note. The flask may burst on account of the increased internal pressure due to the rise of temperature produced by the burning of the phosphorus, but no damage will follow such an occurrence if the operation be carried out exactly as described.)

Thus, although some or, it may be, all of the phosphorus has disappeared, and an entirely new body, the 
white pentoxide of phosphorus, has been produced, there has been no complete destruction nor any creation of matter. The phosphorus still exists, no longer in the free state, but combined with oxygen; some of the latter has also changed from the free to the combined state. To show that the oxygen, or some part of it, has been removed from the air, remove the wire from the cork, and open the mouth of the flask under water. A certain amount of the latter will pass into the flask. (Compare Experiments 79 and 80.) Recork the flask under water, remove it from the water and shake it well. When the oxide of phosphorus has dissolved, remove the cork, and insert a lighted taper. The gas left in the flask will not support combustion, if sufficient phosphorus has been used to combine with all (or most of) the oxygen originally present in the flask.

If, in any other experiment, the total quantity of stuff, whether gaseous, liquid, or solid, taking part in the experiment, together with all the apparatus used, be weighed under similar conditions before and after the performance of the experiment, it will be found that the original weight is unaltered.

Hence, although we may readily bring about the transformation of one kind of matter into another, we can neither destroy nor create matter. This last statement is known as "the Principle of the Indestructibility of Matter", and is one of the most important principles of science.

158. Since the processes of combustion and animal respiration both produce carbon dioxide and watervapour, and are both continually going on in the world around us, it seems likely that the atmosphere will contain some carbon dioxide and some water-vapour. 
Experiment 92. Expose a little clear lime-water on a watch-glass for about twenty minutes. At the end of that time the surface will be covered with a thin bluish-white skin. This consists of precipitated chalk, the particles of which are too fine to break through the water-surface and fall to the bottom of the glass.

The formation of this skin is evidence of the existence of carbon dioxide in the air which has come in contact with the surface of the lime-water.

159. A more pronounced result may be obtained by aspirating a large quantity of air through limewater.

ExPERIMENT 93. Fit a tall gas-jar, containing a little clear lime-water, $L$, with a well-fitting cork, $C$, having two holes bored through it. The tubes $I$ and $O$ pass through the holes and fit them closely.

The tube $O$ is connected with an aspirator (see Fig. 72) or a water-pump. By running water from the aspirator (or through the pump) the air is exhausted from $G$, and the pressure inside the jar thereby lessened. The atmospheric pressure is not altered, and hence air from the room is forced down

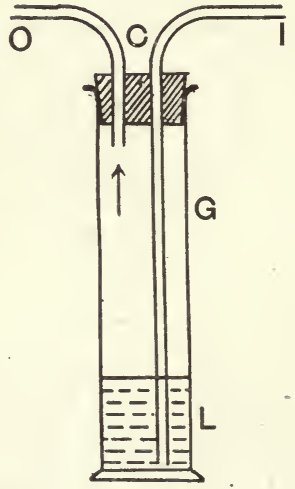

Fig. 74 . tube $I$ and bubbles up through the lime-water. By passing sufficient air through the liquid, a considerable quantity of the white precipitate of calcium carbonate may be obtained.

It will be found that the air must be aspirated through the lime-water for some 20 to 30 minutes in order to produce this result, showing that the proportion 
of carbon dioxide contained in ordinary air is extremely small. As an interesting corollary to the above experiment, perform

EXPERIMent 94. Remove the connection between tube $O$ and the aspirator. Uncork the jar and pour out the lime-water. To clean the inside of the jar from any particles of chalk thàt may adhere to it, pour in a little dilute hydrochloric acid, shake the jar, and pour away the acid. Rinse the jar with several quantities of water, and then pour in a fresh supply of lime-water. Insert the cork and delivery tubes. Blow air from the lungs for a few seconds through tube $I$. The prompt production of a white precipitate affords evidence that the proportion of carbon dioxide in expired air is much greater than that existing in ordinary air.

The observation made in the last experiment explains why the proportion of carbon dioxide in the air of a living-room which is insufficiently ventilated soon becomes considerably higher than that contained in ordinary air.

160. To show the presence of water-vapour in the air we may either cause some of the vapour to condense and hence become visible as water, or may cause some substance which has a strong attraction for water to remove some of the latter from the air.

EXPERIMENT 95. Nearly fill a small clean dry beaker with sodium thiosulphate crystals. (This is the substance known to the photographer as " hypo" from its earlier name "sodium hyposulphite".)

Pour in sufficient water to just cover the crystals. Stir the crystals and water well together with a glass rod for about a minute, and then examine the outside of the beaker.

The moisture which will be found on the exterior 
has been deposited from the air. The sodium thiosulphate in dissolving absorbs heat. Since no heat is specially supplied to the apparatus, the heat absorbed is taken from that originally present in the beaker and its contents, and thus the temperature of the solution and of the vessel is considerably lowered. Hence the air in contact with the exterior of the beaker is also cooled. Now this cooled air cannot retain as much water in the form of vapour as it held before cooling, and as a result the excess of water-vapour is condensed, appearing as small drops on the outside of the beaker.

(Note. The absorption of heat by the solution of the sodium thiosulphate, i.e. by its passage from the solid to the liquid state, should be compared with the heat-absorption which attends the conversion of ice into water, as considered in Chapter $\mathrm{x}$.)

Experiment 96. Expose on a watch-glass a few small lumps of granulated calcium chloride. Examine the chloride at the end of an hour, and compare the appearance of its exterior with that of some which has not been exposed. Put the watch-glass in an exposed position, and examine its contents once more at the end of 24 hours, and again after it has been left for a week.

Substances like calcium chloride, which freely absorb moisture, are termed "hygroscopic". The most hygroscopic substance known is phosphorus pentoxide. (Refer to your observations in Experiment 82 on the behaviour of this substance when left in the open air.) It is hence the best absorbent of water which we possess. To show its great attraction for water, try

EXPERIMENT 97. Remove a small quantity of phosphorus pentoxide from a bottle of the substance, closing the bottle immediately after its removal. 
Drop the pentoxide quickly into a beaker containing a little water. The combination of the pentoxide with the water is attended with so much heat that a little water is converted into steam, and a sharp hissing noise is heard.

The next experiment affords another striking example of hygroscopy.

EXPERIMENT 98. Make a weak solution of cobalt chloride. Write your name on a clean sheet of paper, using a clean nib and the cobalt chloride solution as ink. Allow the writing to dry, and it will be found that the characters will at best be but very faintly visible. Now warm the paper carefully over a small Bunsen flame. The writing will gradually appear in bright blue characters. Dry cobalt chloride has a bright blue colour; when this substance is moistened, it has only a very faint pinkish tinge. Allow the blue writing to remain in the open air, and examine it from time to time. Account for any change which occurs.

Cobalt chloride solution was one of the earliest of the so-called "invisible inks" to be discovered.

161. The foregoing experiments have shown clearly that carbon dioxide and water-vapour exist in the atmosphere, and also that they are continually being produced by the process of combustion and animal respiration. Water-vapour is further being continually given off from the surfaces of all exposed sheets of water under ordinary circumstances, and it is from this source that the major portion of the aqueous vapour present in the air is obtained.

Experiment 99. Nearly fill a small beaker with water. Weigh the beaker and its contents carefully and mark the level of the water by gumming a strip of paper vertically on the outside of the beaker, and 
drawing a short pencilled line on the paper at the water-level. Leave the beaker exposed to the air for a day in a part of the room as free from draughts as possible. At the end of that time again mark the water-level and weigh the beaker and water. Repeat this daily for several days.

An instructive method of recording the observations made so that they may be readily compared, is that of

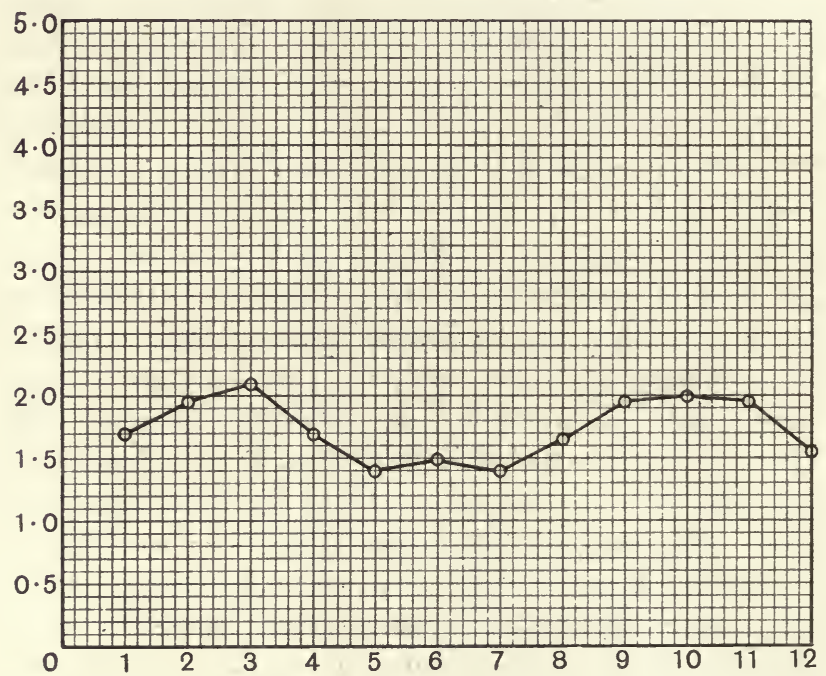

Fig. 75 .

plotting the values on squared paper. The plotting is performed as follows :

On a sheet of squared paper draw horizontal and vertical lines intersecting at $O$, as shown in Fig. 75, Along the horizontal line mark off a scale of time, starting from $O$, the consecutive numbers on this scale representing the numbers of days since commencing 
the experiment. Starting again from $O$, mark out similarly a scale of weights on the vertical line, the numbers indicating grams and fractions of a gram. To obtain the point corresponding to the first pair of observations taken, note the point on the vertical scale which represents the loss of weight experienced by the water during the first day. From this point draw a faint horizontal line to meet the vertical line from the figure " 1 " on the time scale. Make a small dot at the intersection of these lines and surround it by a small circle. Each successive point is obtained in similar fashion, the horizontal line from the point on the scale of grams which indicates the loss of weight during any given day being drawn to meet the vertical line from the number representing the corresponding number of days that have elapsed since the beginning of the experiment. Join the various points thus found by short straight lines as indicated in the figure.

(Note. The results obtained will be more strictly comparable if sufficient water be added each day to that remaining in the vessel to compensate exactly for the loss of weight noted.)

This experiment may well be performed in conjunction with

Expertment 100. Fig. 76 represents an instrument called a "wet and dry bulb hygrometer" (moisture-measurer).

One of the thermometers is an ordinary thermometer mounted on

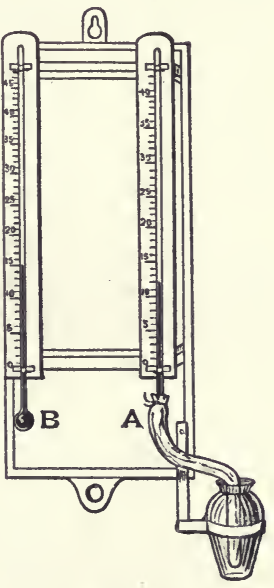

Fig. 76 . a wooden frame, and the other is an exactly similar 
thermometer; the bulb of which is surrounded by loose strands of lamp-wick, the other ends of which dip into a vessel which is kept nearly filled with water. By means of this arrangement the bulb of the latter. instrument remains continually moist.

Take the readings of both at as nearly as possible the same instant. It will be found that the temperature recorded by the dry bulb thermometer is higher than that shown by the other. The explanation of this difference is as follows :

Water is always evaporating from the threads in contact with the bulb of the latter at a rate which depends on the dryness of the air around the bulb. We have already shown in Chapter $x$ that when evaporation occurs, heat is absorbed. In the case of the hygrometer, there is no supply of heat from an outside source which can compensate for the heat thus absorbed, and hence the heat necessary is supplied by the objects in contact with the water which is being vaporised, i.e. by the bulb of the thermometer. As a result, the latter is cooled below the temperature of the room, and hence the wet bulb thermometer registers a lower temperature than its fellow.

On a very dry day, this difference may be considerable, while if the atmosphere be very moist, it may amount to less than $1^{\circ} \mathrm{C}$.

A series of readings of the wet and dry bulb thermometers should be taken daily at the same time as the weighing of the beaker of water in Experiment 99 . The average differences of temperature during the intervals between the times of reading may be assumed to be approximately the mean of each pair of consecutive difference readings.

On the same sheet of squared paper as used in 
Experiment 99, plot the values obtained showing the relation between these average differences of temperature and the time, using the scale of time adopted in the previous experiment. The sort of weather experienced between each pair of consecutive readings should also be noted, i.e. whether fine, cloudy, wet, foggy, or otherwise.

\section{Exercise for Student.}

Try to trace, and to account for, any relation between the average temperature differences shown by the hygrometer and the loss of weight sustained by the water during the same interval.

162. Tables have been constructed by reference to which it is possible to obtain, for any given pair of simultaneous readings of the wet and dry bulb thermometers, an idea of the actual percentage of watervapour present in the atmosphere in the neighbourhood of the instrument at the time of observation. As it is unlikely that the student will have access to such a set of tables, we will next describe a chemical method of determining the weight of water-vapour present in a given quantity of air.

Experiment 101. Fit up the apparatus shown in Fig. 77. Before commencing the experiment, weigh each U-tube with its contents separately, and then quickly fix them in position. See that all joints and corks fit closely.

Turn the stopcock so that a slow stream of water is produced. Continue this till at least 10 litres of water have run from the aspirator. Catch the water as it leaves the tap in a large measuring vessel. When the latter is filled to its topmost graduation, turn off the tap, quickly pour out the water from the measuring 
vessel, replace the latter, and at once re-start the outflow of water. Should the aspirator be of less than 10 litres capacity, disconnect it when empty, rapidly refill it, and replace it. Repeat this refilling till the requisite quantity has been run out.

The volume of water collected is equal to the volume

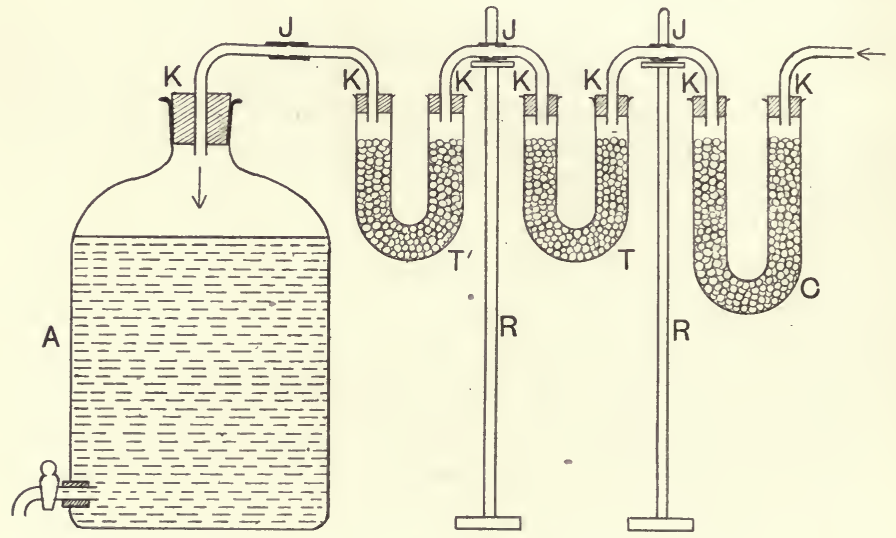

Fig. 77.

$A=$ large aspirator fitter with stopcock.

$C=$ large $\mathrm{U}$-tube filled with pumice-stone moistened with pure sulphuric acid.

$T, T^{\prime}=$ small $U$-tubes filled similarly to $C$.

$K, K \ldots=$ tight-fitting rubber stoppers.

$R, R=$ retort stands.

$J, J, J=$ close-fitting rubber joints.

of air which has passed through the drying-tubes. Disconnect the latter, and quickly re-weigh each separately.

The increase in weight of $C$ is the weight of the moisture present in the volume of air passed through it. Any increase in the weight of $T$ beyond, say, $.005 \mathrm{~g}$., 
shows that the air passage has been too rapid, and that all the moisture in the air has not been absorbed in $C$. $T^{\prime}$ should also show no appreciable gain in weight. Its function is to prevent backward diffusion of the moistureladen air from the aspirator into either $T$ or $C$.

It will be found that, even when 10 litres of air have been passed through the tubes, the gain in weight is small, and hence every care must be taken in order to obtain a good result.

Further, it must be clearly understood that, by this method, only the average amount of moisture present in the air around the apparatus during the whole time of the experiment can be ascertained, and not the actual amount present at any given instant.

163. Besides the substances already mentioned there are numerous other bodies found in very small quantities in the air, especially in the neighbourhood of towns and cities. The table below gives the percentage composition by volume of what may be considered an average sample of ordinary air.

$$
\text { Per cent. }
$$

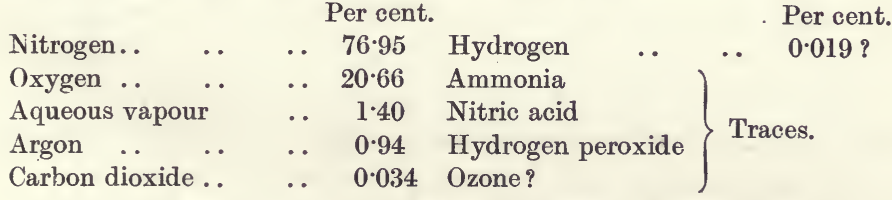

In addition to the above, traces of sulphur compounds, such as sulphur dioxide and sulphuretted hydrogen, are found in the air of towns where coal and coal-gas, both of which contain a little sulphur, are used. Further, ordinary air is never entirely free from small solid impurities, such as dust and soot particles, and small living organisms known as bacteria.

164. Since carbon dioxide is being continually formed by the respiration of animals and by the burning of fuel, H. D. S. 
the total quantity produced during the period since animals first came into existence and fire was discovered must be very great. A corresponding amount of oxygen must have been removed from the air in order to combine with the carbon and thus form the carbon dioxide. It would therefore seem probable that the percentage of carbon dioxide in the air would increase, and that of oxygen would diminish, in the course of ages. We believe, however, that these percentages do not appreciably vary, and two factors are concerned in this unchangeableness of the amounts of the two gases to be found in the atmosphere. Firstly, the carbon dioxide forméd at any spot is rapidly diffused through the surrounding air, and the total mass of the Earth's atmosphere is so enormous that it would take an exceedingly long time for the proportion of carbon dioxide found in an ordinary sample of "fresh" air to be altered to a sufficient extent for the change in amount to be detected even by the most delicate methods at our disposal. Secondly, the carbon dioxide -a waste product of the animal organism-provides food-material for plants. Their structure is such that they are capable of absorbing carbon dioxide from the air under suitable conditions, using the carbon to build up their tissues, and returning oxygen to the air.

Experiment 102. Fit up the apparatus shown in the annexed figure. The flask should have a capacity of about two litres and should be entirely filled with ordinary tap-water. The common water-weeds are the best plant material.to place in the water as shown. Put the whole apparatus in bright sun-light with the test-tube, filled with water, fixed in the position indicated in the figure. Bubbles of a gas will be slowly disengaged from the surface of the leaves 
and, in time, sufficient of this gas will collect in the upper end of the test-tube for its nature to be tested. Remove the tube, placing the thumb under its mouth to prevent the entrance of air, invert it, and apply a glowing splinter of wood to the mouth of the tube. The wood will burst into flame - a result which we learnt in Experiment 44 to be characteristic of the gas oxygen.

The plant has acted upon the carbon dioxide dissolved in the tapwater and has produced oxygen as a waste-product. To emphasise the part played by the dissolved carbon dioxide the experiment may be repeated, the flask being filled with (a) tap-water which has been boiled for twenty minutes and then quickly cooled in a flask of which the mouth is lightly stoppered with a plug of

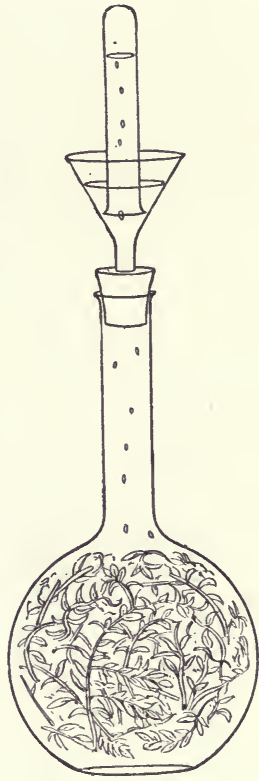

Fig. 78 . cotton-wool; $(b)$ some water through which carbon dioxide has been allowed to bubble for several minutes.

\section{Exercise for Student.}

Account for the varying behaviour of the water-weed under the different conditions suggested above.

165. It must not be inferred that plants under all circumstances evolve oxygen. Carbon dioxide is produced by the activity of a living plant in certain conditions. As an example of this, the following experiment is instructive. 
Experiment 103. Place a layer of moistened sawdust at the bottom of a gas-jar. On this drop some French or haricot beans, or other large seeds. Cork up the mouth of the flask firmly and store it in a warm place in the dark for some days. Examine the jar from time to time, and when the seeds have germinated, allow them to grow for two or three days. At the end of that time, uncork the flask, and test the contents by inserting (a) a drop of lime-water on the end of a glass rod ; $(b)$ a lighted taper. The air of the jar will afford evidence of the presence of a considerable proportion of carbon dioxide when tested in this way.

The decay of all forms of plant life is of the nature of slow combustion and is attended by the formation of much carbon dioxide.

Expertment 104. Fit up two large jars as indicated in Fig. 74. Half fill one with moist decaying vegetable matter, such as dead leaves. Fit the cork and tubes to the jar and allow it to remain undisturbed in a warm place for about a day. At the end of that time connect the outlet tube with the inlet tube of the second jar, having previously poured a quantity of clear limewater into the latter vessel. Attach an aspirator or pump to the outlet of the second jar and cause a gentle stream of air to pass through the apparatus for a short time. The production of turbidity in the lime-water in a much shorter time than was the case in Experiment 93 affords evidence that the air in the jar containing the decaying matter is considerably richer than ordinary air in carbon dioxide.

166. The water-weed in Experiment 102 was said to obtain a supply of carbon dioxide from the water in the flask. In addition to carbon dioxide, the other constituents of the air also dissolve in water to some extent. 
Experiment 105. Fill a flask of about 1 litre in capacity with water. Push in, with a screwing motion, a tight-fitting rubber stopper, carrying a bent deliverytube as shown in Fig. 79. Pushing in the stopper should result in filling the delivery-tube with some of the water from the flask. The upper end of the tube should be flush with the bottom of the stopper. Insert the free end of the tube under the mouth of the graduated vessel, which may well be a 100 c.c. measuring tube or cylinder. Heat the water to boiling-point. Bubbles of gas will be formed as the liquid becomes hot,

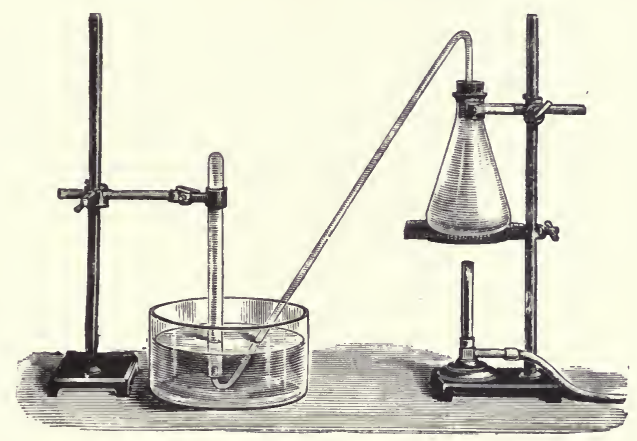

Fig. 79 .

and these will rise to the top of the flask, escaping later via the tube into the collecting vessel. Continue the heating for about ten minutes after the water has reached boiling-point. At this stage remove the measuring tube to another part of the trough and then turn out the burner. Observe carefully what happens as the water in the flask cools.

\section{Exercise for Student.}

Account for all observations made as the water cools. 
The nature of the gas which has been collected may be tested after allowing the water and gas in the measuring tube to cool. We already know that caustic potash solution readily absorbs carbon dioxide. After reading the volume of gas collected in the tube, with the water inside and outside the tube at the same level, insert a small stick of caustic potash under the mouth of the tube, close the mouth with the thumb and remove the tube from the water. Shake the tube till all the potash has been dissolved and then remove the thumb under water. Read off the volume of gas remaining. The difference between the two volumes will be the amount of carbon dioxide contained in the gas collected from the water. Part of this carbon dioxide was dissolved in the water as such, and part in the form of compounds which are decomposed by heating them to the temperature of boiling water. (See Part II, Hardness of Water.)

The remainder of the gas in the tube consists almost entirely of oxygen and nitrogen. To determine roughly their relative amounts, put about $0.1 \mathrm{~g}$. of pyrogallic acid-the "pyro" of the photographer-into a filterpaper, roll it up tightly, and insert it under the mouth of the tube. Close the tube as before with the thumb and remove it from the water. The pyrogallic acid will dissolve readily and, in the presence of the caustic potash already in solution, will quickly absorb the oxygen, if the tube and its contents be gently shaken. The liquid will assume a deep brown tint as a result of this absorption. After shaking for about five minutes remove the thumb under water and again read off the volume of gas which remains in the tube. This may be taken as representing the nitrogen in the gas originally collected, while the volume which 
has disappeared as the result of the treatment with the pyrogallic acid is that of the oxygen present. Compare the volume-ratio between the nitrogen and the oxygen, as thus obtained from water, with that in which they exist in the air.

\section{Exercise for Student.}

Attempt to account for any difference between the two volumeratios mentioned above.

We shall be able to make use of the observations recorded in this experiment in later work dealing with the nature of the two substances, air and water.

167. That many metals, more especially iron, rust when exposed to the action of water is a familiar everyday experience. We will next try to show the conditions under which rusting may or may not take place.

Experiment 106. Completely fill a jar with recently boiled and cooled distilled water. Into this drop a piece of clean bright iron. A new screw which has been well washed with soap and water and thoroughly rinsed will serve excellently. Allow the screw to remain in the tightly stoppered jar for several days and examine its appearance daily.

Cover the bottom of a second jar with a layer of small lumps of calcium chloride. Cork up the jar and put it on one side for a day. At the end of that interval hang a second screw similarly cleaned and then dried in the jar by means of a piece of string kept in position by the cork, in such a way that the screw does not touch the chloride layer. As before, examine the appearance of the screw daily for some days.

Drop enough tap-water into a third jar to cover the bottom to the depth of about one-tenth of an 
inch. A third screw is to be dropped into this jar, which is then securely stoppered. Examine the appearance of this screw at the same times as the others are examined.

From the observations made in the above experiment it will probably be inferred that neither pure water, free from dissolved gases, nor dry air, can alone cause the formation of rust. Exposure to the combined effects of moisture and air, however, readily brings about this change. The rust is of somewhat variable composition, being a mixture of the brown oxide of iron, called "ferric oxide", with a compound of this oxide with the elements of water, called "ferric hydroxide". It seems probable that the carbon dioxide of the air plays a part in the rusting, since the addition of a little solution of caustic potash to the water will prevent the formation of the layer of rust.

168. The gases of the air dissolved in water are of considerable importance to the maintenance of life. We have already noted the part played by the dissolved carbon dioxide in the vital processes of water-plants. The dissolved oxygen has an equally important function in that aquatic animals are dependent upon it for their supply of this gas, which is as necessary for their existence as for that of ourselves and all other land animals. The gills of a fish are composed of delicate membranes through which the air dissolved in the water is able to pass, the fish using the oxygen and returning the other gases and the carbon dioxide produced by the oxidation of its food to the water in which it lives. If placed in recently boiled and cooled water, a fish rises to the top of the water in a gasping condition and speedily dies from suffocation if it be not removed into some ordinary water. Again, if a number 
of fish be kept in a limited quantity of water, they soon die, since they quickly remove the dissolved oxygen and the water becomes too foul for their continued existence to be feasible, just as a closed room in which a number of people are living becomes unfit for habitation unless some method of supplying fresh air be adopted.

\section{QUESTIONS}

1. Suggest a method of determining roughly the percentage volume of carbon dioxide in respired air.

2. The air of a deep well or mine is sometimes tested before men go down by lowering a lighted candle at the end of a rope. What information concerning the state of the air is obtained by this means ?

3. How would you try to show that the air of a living-room contained a larger proportion of carbon dioxide than the outside air ?

4. The density of carbon dioxide is $1 \frac{3}{8}$ times, and that of nitrogen $\frac{7}{8}$, that of oxygen. Explain why the atmosphere is not composed of a layer of carbon dioxide near the Earth's surface, with consecutive layers of oxygen and nitrogen above.

5. Give an account of the part played by each of the various constituents of the air in plant and animal life.

6. Describe a process of estimating the proportion of oxygen in a sample of air which neither involves the direct oxidation of iron nor of phosphorus.

7. Name the five principal constituents of ordinary air, and describe experiments by means of which the presence of any two of them in air may be shown.

8. How can the presence of water-vapour in the air be accounted for ?

9. What do you understand by the statement that iron is an element? Classify the following bodies as elements or compounds: tin, water, chalk, nitrogen, argon, iron rust.

10. The specific gravity of nitrogen is $\frac{7}{8}$ that of oxygen. Using the percentage volumes given in Chapter XIII, find the percentage weights of nitrogen and oxygen in air. 
11. Describe how you would arrange an experiment to demonstrate that the products of combustion of ordinary coal-gas weigh more than the coal-gas burnt.

12. Cooking-salt frequently becomes very daimp when exposed to the air, while prepared table-salt under similar circumstances remains dry. Suggest reasons for this difference in behaviour.

13. One cubic centimetre of steam weighs $\cdot 000805 \mathrm{~g}$., and the same volume of air weighs $00129 \mathrm{~g}$. Calculate the weight of watervapour in 100 litres of air, if the air contains 1.5 per cent. by volume of water-vapour.

\section{CHAPTER XV}

\section{OXYGEN}

169. Nitrogen may be obtained from the air in a state of comparative purity by any of the several means described in Chapter XIII. No simple method has been devised whereby the oxygen may be directly separated in similar fashion. It is possible to show indirectly that this gas is actually present in the atmosphere by carrying out somewhat lengthy and troublesome experiments. The best known of these, ascribed to the famous French scientist, Lavoisier, and used by him to show that the ideas prevalent in his day concerning the nature of the changes involved in rusting and burning were entirely fallacious, deserves description.

In the bulb of the curiously shaped retort shown in the adjacent figure, Lavoisier poured a quantity of mercury, which had been carefully weighed. The end of the bent neck of the retort dipped under the surface of mercury in the trough, and the air in the retort and 
that in the bell-jar were in communication with one another via this neck. The retort was then heated cautiously by means of the furnace to a temperature just short of that at which mercury boils. After some hours the surface of the mercury became covered with reddish specks, and a simultaneous rise in the level of the mercury within the bell-jar took place. The heating was continued for a considerable time, and at length no more of the red substance seemed to be formed and the mercury rose no further in the bell-jar. To make quite certain that no more of the air could be caused to

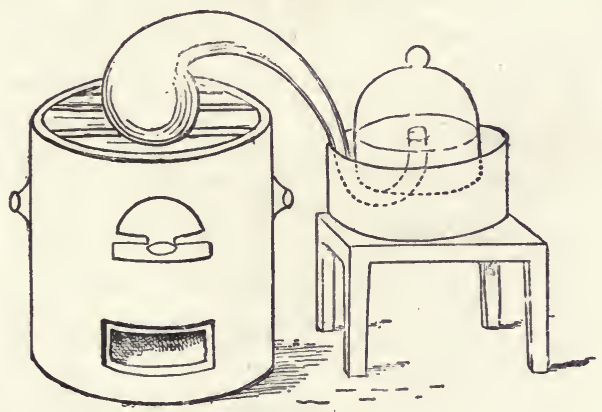

Fig. 80 .

disappear, the heating was kept up for over eleven days. The apparatus was then allowed to cool and the decrease in volume of the air contained in the bell-jar was measured as carefully as possible. All the red powder was next removed from the surface of the mercury in the retort, placed in a smaller retort, and heated strongly. A gas was evolved and this was collected in a graduated measuring jar. Mercury alone was left in the smaller retort as a result of this treatment and the volume of the gas given off was found to be exactly 
equal to the decrease in the volume of the air in the earlier part of the experiment. The gas left in the bell-jar was shown to be irrespirable and a non-supporter of combustion, while that obtained by heating the red powder gave evidence of possessing the opposite characteristics to a marked degree.

The repetition of the first part of Lavoisier's experiment cannot be readily carried out, but we may test the latter portion with ease by repeating Experiment 44, since the red oxide of mercury used in that experiment is no other than the red powder formed in Lavoisier's experiment. The change of the mercury into the red powder is expressed by the chemical equation:

Mercury + oxygen = mercury oxide.

The reverse change brought about in the later stage of the same experiment is written thus:

$$
\text { Mercury oxide = mercury }+ \text { oxygen. }
$$

A somewhat similar method is made use of to obtain large supplies of oxygen from the air by the manufacturing process known as "Brin's process". In this, barium oxide is heated in air under pressure, when it combines with oxygen to form barium dioxide. This substance is then heated at the same temperature under a very much reduced pressure, and the oxygen with which it combined previously is set free.

Equations :

Barium oxide + oxygen $=$ barium dioxide

Barium dioxide = barium oxide + oxygen.

170. Oxygen may also be obtained by the action of heat on many other oxides.

Experiment 107. Treat some red lead oxidecommonly known as red lead-in a similar fashion to 
that in which the mercury oxide was treated in Experiment 44. Test for the evolution of oxygen with a glowing splint. Examine the product left in the tube. It is known as lead oxide or litharge.

Red lead oxide $=$ yellow lead oxide + oxygen.

Experiment 108. Into a small tube made of hard glass-a test-tube will not stand the temperature necessary - put some black manganese dioxide. Heat this strongly with a powerful Bunsen flame, and test from time to time with a glowing splint. At a red heat oxygen may be recognised. Note any change in the powder after no more oxygen seems to be produced.

Manganese dioxide $=$ brown oxide of manganese + oxygen.

171. Many other substances besides oxides give off oxygen on being heated, especially nitrates and chlorates.

Experiment 109. Heat some nitre in a small hard tube. Observe the behaviour of the crystals and test for the evolution of oxygen. Continue the heating till no more gas is given off.

Potassium nitrate $=$ potassium nitrite + oxygen.

(nitre or saltpetre)

Experiment 110. Heat some potassium chlorate in a test-tube, taking note of the behaviour of the compound and of the amount of heating required to cause oxygen to come off. As before, continue heating till no more change occurs.

Potassium chlorate $=$ potassium chloride + oxygen .

172. When oxygen is required in quantity for laboratory work with the gas, a mixture of two of the bodies named above is in common use. 
ExPERIMENT 111. To a watch-glassful of potassium chlorate add about one-fifth its bulk of manganese dioxide. Mix the two substances well together and transfer the mixture to a test-tube. Fit the test-tube with a well-fitting cork, through which passes a doublybent delivery tube. Complete the fitting up of the apparatus as shown in Fig. 81. Heat the tube gently by making the Bunsen flame of such a height that the tip just touches the under surface of the test-tube. Start the heating at the end of the mixture nearer the

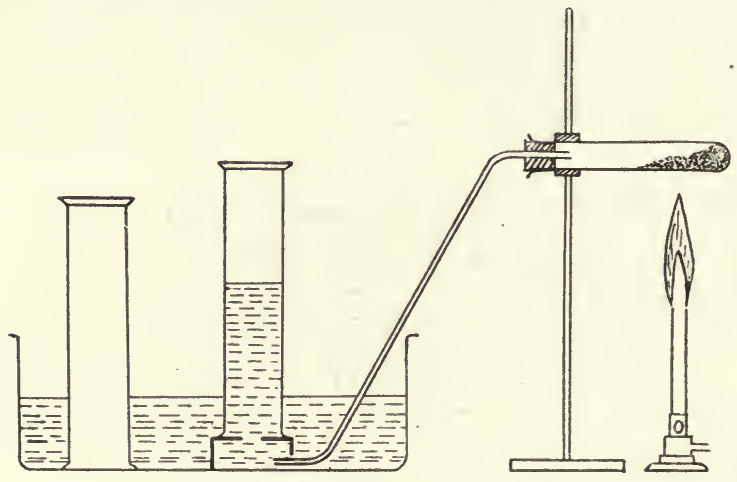

Fig. 81 .

mouth of the test-tube and, when all the oxygen has come off from this portion, gradually transfer the flame toward the sealed end of the test-tube. Oxygen will be quickly given off and several jars may be obtained from the quantity of mixture suggested for use.

173. On consideration of the temperature required to procure oxygen from this mixture, it will probably be noticed that it is given off much more readily from the mixture than from either of the two substances separately. Careful investigation of the action which 
occurs has shown that the oxygen is supplied by the potassium chlorate and that the manganese dioxide is apparently unaltered during the experiment, being recoverable from the residue in the test-tube unchanged in quantity. Many similar reactions are known in which a substance causes the result of a reaction to be brought about more readily but itself appears to undergo no change. The learned name given to this phenomenon is " catalysis", which means "decomposition by contact". Because we have this name in our memories, however, we must not think that we know more about the peculiar nature of the action. Up to the present no perfectly satisfactory explanation of why the manganese dioxide should help the potassium chlorate to give up its oxygen has been put forward.

174. With the oxygen obtained as above, the following experiments should be performed.

EXPERIMENT 112. Insert a glowing splint of wood into one jar. Observe the brightness of the flame produced. Into the same jar, if all the oxygen be not used up, introduce a lighted candle on the end of a piece of copper wire. Observe the decrease in size and the gain in brightness of the flame.

\section{Exercise for Student.}

How do you explain the smaller size of the flame of the candle when it burns in oxygen?

Experiment 113. Burn coal-gas from a small glass jet in a second jar of oxygen by means of the arrangement indicated in the figure. Note any changes in the nature of the flame. Should the heat produced be sufficient to close the jet by melting the edges of the glass together, another tube may be prepared 
with a small roll of platinum foil fused into the end of the tube to form the jet.

Experiment 114. Place a piece of charcoal, about the size of a hazel-nut, in a deflagrating spoon, and lower the spoon into another jar of oxygen, after igniting one corner of the charcoal in the Bunsen flame. When all burning has ceased, remove the spoon, quickly pour in some water, and at once cover the mouth of the jar with a glass plate. Shake the jar and its contents well. Into the liquid thus obtained drop a little

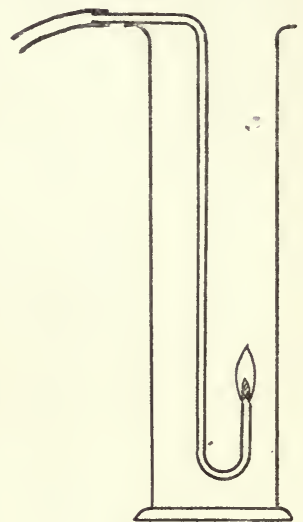

Fig. 82 . litmus solution. The bluish solution will become of a dull purplish-red colour.

By burning a second piece of charcoal in a fourth jar, it may be shown, by inserting a lighted taper and by shaking up a little lime-water in the jar, that the gas left in the jar, after the combustion has taken place, is carbon dioxide.

$$
\text { Carbon + oxygen }=\text { carbon dioxide. }
$$

Experiment 115. Burn a small piece of brimstone or sulphur in similar fashion in a fifth jar. Observe the production of white fumes, evidence that a certain amount of a substance called sulphur trioxide has been formed during the burning. The main product of the action, however, is a colourless gas, sulphur dioxide, and the presence of this may be detected by cautiously smelling the contents of the jar after combustion has ceased. Shake up with water and 
test with blue litmus solution as in the previous experiment.

ExPERIMENT 116. Burn a small piece of phosphorus in another jar, carefully observing all the precautions mentioned in Experiment 78 during the handling of ${ }^{\circ}$ the substance. After the jar has cooled, shake up with water and test with litmus as in the two last described experiments.

Equations :

Sulphur + oxygen $=$ sulphur dioxide.

$($ Sulphur + oxygen $=$ sulphur trioxide. $)$

Phosphorus + oxygen $=$ phosphorus pentoxide.

175. As a common result of the testing with blue litmus solution in Experiments 114, 115, and 116 it will have been noticed that the products of the combining of the three elements, carbon, sulphur, and phosphorus, with oxygen all possess, when dissolved in water, the power of turning blue litmus red. Similar observations were made by Lavoisier. Bodies which have this property are termed "acids", so he gave the name " oxygen" (from Greek, oxus, sharp or acid, and gennao, to produce) to the gas which he thought was a characteristic constituent of all acids. We now know that his idea is incorrect, but we retain the name oxygen for the gas.

EXPERIMENT 117. Sodium is a peculiar metal which is kept in bottles containing paraffin oil in order to protect the metal from the oxygen of the air. Remove a small piece, not larger than a pea, with $d r y$ crucible tongs from a bottle and place it on some dry filterpapers on a dry bench. Absorb the greater part of the adhering oil by gentle pressure with the papers and put the sodium into a dry deflagrating spoon. Avoid H. D. s. 
touching it with the fingers, unless these are quite dry. Start the burning of the scdium by heating the spoon in a Bunsen flame. (Ignore the flame first produced, since this is only the paraffin not removed by the filterpaper burning away.) Lower the spoon into a jar of oxygen and allow it to remain till all burning has ceased. Observe the energetic nature of the combination of the metal with the gas. When the whole apparatus is quite cold, shake up some water in the jar, and dip the end of the spoon into the water. A vigorous action will probably be noticed when this is done, accompanied by a hissing noise. No danger attends this, if the piece of sodium used be not larger than suggested. Test the action of the solution produced upon red and blue litmus papers. The latter will be unaffected, while the red paper, the colour of which has been produced by allowing a small quantity of acid to act upon the colouring-matter, is rendered of a bright blue tint. This result shows that Lavoisier was somewhat hasty in his conclusion that oxygen was typically an " acid"producer". The main product of the combustion of sodium is an oxide called sodium peroxide. When this acts upon water, part of the oxygen is set free, and the remainder of the substance behaves as if it were an oxide with a lesser proportion of oxygen, called sodium oxide.

Sodium + oxygen $=$ sodium peroxide.

176. The reactions of the various oxides upon water may be thus represented :

$$
\begin{gathered}
\left.\begin{array}{c}
\text { Carbon dioxide }+ \text { hydrogen oxide }=\begin{array}{c}
\text { hydrogen carhonate } \\
\text { (carhonic acid) }
\end{array} \\
\text { (water) } \\
\text { Sulphur dioxide + " }, " \quad \begin{array}{c}
\text { hydrogen sulphite } \\
\text { (sulphurous acid) }
\end{array} \\
\text { = hydrogen sulphate } \\
\text { (sulphuric acid) }
\end{array}\right\}
\end{gathered}
$$


Phosphorus pentoxide + hydrogen oxide $=$ hydrogen metaphosphate

(water) (metaphosphoric acid)

Sodium oxide $+\quad " \quad=\quad \begin{gathered}\text { sodium hydroxide } \\ \text { (caustic soda) }\end{gathered}$

Bodies like caustic soda, which, when dissolved in water, are capable of producing the reverse change in the colour of litmus to that effected by the action of acids, are termed "alkaline" (from the Arabic $a l$, the, and qali, the ashes of a plant, known as glasswort, from which soda was first obtained).

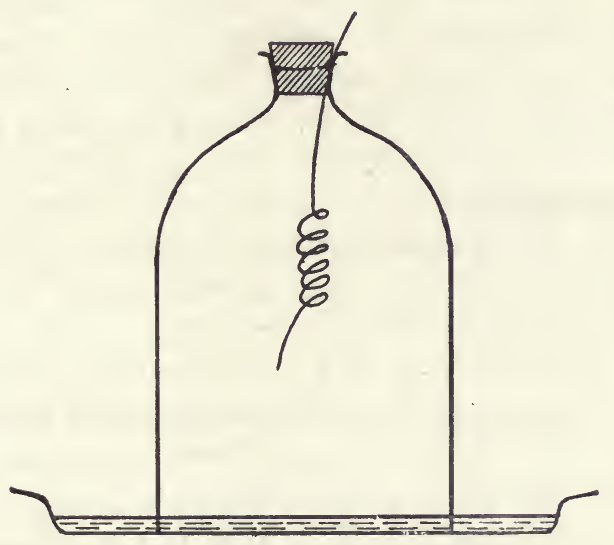

Fig. 83.

177. Substances which are not looked upon ordinarily as combustible may often be burnt in oxygen. Iron is used in the construction of fire-grates and for many other purposes where strength and resistance to the action of heat are desirable properties. That it would be no longer of service to man in this way if the atmosphere consisted of oxygen alone is demonstrated by

Experiment 118. To the end of a piece of watch or clock spring affix a short length of wax-taper by 
means of the fine iron wire used for tying up flowers. Wire the other end of the spring to a cork, which fits the neck of a bell-jar. Fill the bell-jar with oxygen, and stand it over water in a shallow plate. (The filling may be done well enough for all practical purposes if a rubber tube, attached to the end of the delivery tube of an apparatus in which oxygen is being generated, is allowed to dip to the bottom of the jar, while the latter is standing in position on the plate, and the gas passed in for about three minutes in a steady stream.) When the apparatus has been prepared as above, light the taper, and while it is still well alight, plunge taper and spring into the bell-jar. The great heat produced by the burning of the taper in oxygen will be sufficient to start the combustion of the iron. Vivid glowing of the molten ball of metal formed at the end of the spring, accompanied by showers of bright sparks, will be observed as the iron burns away. The sparks consist of a black oxide of iron, and they may be found at the bottom of the water after the completion of the burning. Pour the water from the plate into a jar and see that as much of the iron oxide as possible is transferred to the same jar. Shake the jar and its contents well and test whether the liquid left in the jar has any effect on either red or blue litmus. (Should any positive result of these tests be obtained, repeat the experiment with water previously tested with regard to its action on litmus and found to be neutral, i.e. neither acid nor alkaline.)

178. We have now learnt enough of the qualities of oxides to be able to classify them into certain groups. Thus we have acidic oxides-those which form acids when combined with the elements of water. Contrasted with these are the basic oxides of which we have 
sodium oxide and the black oxide of iron as examples. Should the basic oxide, when combined with the elements of water, form a hydroxide soluble in water, this solution is alkaline in reaction. Those hydroxides insoluble in water, such as ferric hydroxide, the substance formed when the brown oxide-the chief constituent of iron rust-is caused to combine indirectly with the elements of water, cannot, of course, show any action upon litmus, and hence such hydroxides are not alkalis. They are termed simply bases. Most of the metallic hydroxides are of this class. We shall return to this method of classification of the oxides later.

Water itself-hydrogen oxide--is neutral in reaction, and is hence known as a neutral oxide. Further, when two or more oxides of a metal are known, and the highest gives off oxygen under suitable conditions, this oxide is termed a peroxide (Latin, per, very).

179. Several examples of chemical reactions have now come under our notice, and we have had evidence that a considerable amount of energy must be contained within the substances taking part in the various changes studied. This form of energy is called "chemical" energy. During many of the actions chemical energy has been degraded into heat, e.g. large quantities of heat were produced during the combustions of the different substances burnt in oxygen. On the other hand, heat had to be supplied in order to maintain some of the changes, such as those in which mercuric oxide was made from its elements, and vice versa. Here heat energy is changed into chemical energy. Reactions of the first class, in which heat is evolved, are termed "exothermic", while those in which the continuous provision of heat is required for the completion of the change are called "endothermic" reactions. 
180. In our previous work we have considered the unit particle involved in questions dealing with energy to be the molecule. For the purpose of explaining the phenomena of chemistry, a new unit is needed. Molecules are supposed to consist of exceedingly small particles, termed "atoms" (Gr., $a$, without; tome, cutting), those substances the molecules of which have only one kind of atom being the elements, while those possessing different sorts of atoms in each molecule are what we have previously termed compounds. Chemical energy resides chiefly in the atoms. The formation of new combinations of these, resulting in the production of compounds, and the breaking up of combinations already existent, simpler compounds or elements being thus brought into being, cause changes in the quantity of chemical energy present before and after the actions are brought about. In the former kind of change, "combination" or "synthesis" is said to occur, while an action of the latter kind is termed a "decomposition" or " analysis".

181. The equations we have hitherto used to represent in brief the course of a chemical reaction are not, strictly speaking, "chemical " equations. In these latter, much more information is provided as to the probable nature of the chemical action concerned than is to be found in our previous equations. Thus the burning of carbon in air or oxygen is indicated by the equation

$$
\mathrm{C}+\mathrm{O}_{2}=\mathrm{CO}_{2} \text {. }
$$

Here $\mathrm{C}$ is a symbol signifying "one atom of the element carbon" ; $\mathrm{O}_{2}$ is a formula for "one molecule of oxygen composed of two atoms of that element"; $\mathrm{CO}_{2}$ is the formula for " one molecule of the compound 
carbon dioxide, consisting of one atom of carbon combined with two atoms of oxygen". Hence such an" equation would be read thus :

One atom of carbon combines with the oxygen in one molecule of oxygen to form one molecule of carbon dioxide.

Further, since we have means of determining the relative weights of the atoms of the various elements, the above equation also shows that 12 unit weights (grams, ounces, tons, ...) of carbon combine with 32 similar unit weights of oxygen to form 44 unit weights of carbon dioxide.

The complete discussion of this matter of chemical symbols, formulae, and equations, and their proper use, is beyond the scope of this book. Hence they will not be introduced in later chapters unless it is desired to lend special emphasis to some important consideration thereby.

The student is advised to abstain from the indiscriminate use of the various symbols and formulae as mere abbreviations for the names of the different substances, in order to avoid the confusion which frequently arises from using them wrongly.

\section{QUESTIONS}

1. Describe as fully as you can a method of obtaining a sample of oxygen from the air.

2. Discuss the suitability or otherwise of the name oxygen for that element.

3. Try to classify all the oxides already mentioned under one of the heads given in the latter part of the chapter.

4. How would you endeavour to find out whether magnesium forms an acidic or a basic oxide? Write out what you think would be the chemical equations representing any changes involved in your method. 
5. Make a list of all the properties of oxygen, both physical - and chemical, with which you are acquainted.

6. Devise a method of finding out if the solution of oxygen itself in water is acid or alkaline.

7. By what tests would you show the nature of the products formed in Experiment 113 ?

8. Classify as far as you can all the chemical reactions already mentioned in the text as $(a)$ cases of synthesis or analysis ; $(b)$ exothermic or endothermic. Try to draw a conclusion from the comparison of the groups into which the reactions fall when arranged under these four heads.

9. Oxygen supports combustion much more vigorously than air. What reason can you suggest for this ?

10. Before Lavoisier's day substances, such as zinc, when burnt, were supposed to give up a peculiar substance, termed "phlogiston", which was restored to them when they were caused to resume the metallic state by any suitable means. Suggest an experiment by which the accuracy of the former part of this statement mav be tested.

\section{CHAPTER XVI}

\section{PHYSICAL PROPERTIES OF WATER}

182. A certain amount of information concerning the nature of water may be obtained from our previous work on that substance. We already know that it is a colourless liquid, with a density of 1 g. per c.c., freezing at $0^{\circ} \mathrm{C}$., and boiling at $100^{\circ} \mathrm{C}$. under an atmospheric pressure of $760 \mathrm{~mm}$. We have, further, made use of its power as a solvent for salt and similar bodies. In the state in which it naturally occurs, water is never quite pure, and the water which we ordinarily make use of for washing and drinking purposes contains small quantities of other substances dissolved in it. With 
the gases thus held in solution we have already dealt, and our next study will be the question of the solid matter similarly present and the methods in general use for its removal when required.

Experiment 119. Let six drops of water from the tap fall upon a carefully cleaned watch-glass. Place the glass upon a tripod stand, a piece of fine wire gauze being inserted under the glass. Heat the glass and its contents with a Bunsen flame of such a height that the tip of the flame just touches the under surface of the gauze. Continue the heating until the water has almost entirely disappeared, and at this stage remove the burner. The hot gauze will complete the evaporation of the last traces of the liquid. Examine the residue at the bottom of the watchglass. This consists of the

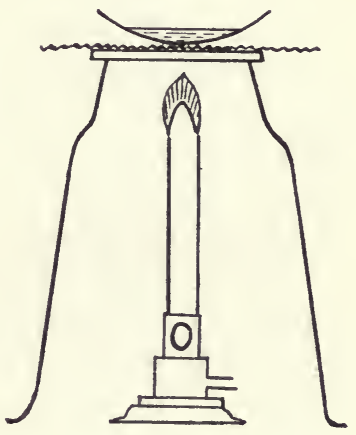

Fig. 84. solid matter previously in solution in the water. Preserve the glass for purposes of comparison with results obtained in later work.

183. To obtain a clearer idea of the relative quantity of solids thus dissolved in the tap-water, perform

Experiment 120. Weigh a clean dry porcelain dish to the nearest milligram. Into the dish introduce, by means of a pipette, 25 c.c. of tap-water. Place the dish upon a water-bath. (The usual laboratory apparatus may be replaced without loss of efficiency by a beaker, of which the mouth is of such a size that the dish will just rest upon it.) Heat the water in the 
bath to boiling. The heat evolved during the condensation of a portion of the steam on the bottom of the dish will cause the water in the dish to evaporate slowly. When it has all disappeared run in another 25 c.c. and again evaporate to dryness. Similarly treat two further quantities of 25 c.c. Allow the dish to cool and. weigh it once more. The increase in weight will be that of the solid matter dissolved in 100 c.c.-- for all practical purposes the weight of this may be taken as $100 \mathrm{~g}$.- of the tap-water.

Should other supplies of water be readily available, the percentage of dissolved solids may be found in similar fashion. River-water, spring-water and rainwater can usually be tested without much difficulty with regard to obtaining supplies of any one of them.

184. In cases where the quantity of dissolved solid matter in a water-supply is not excessive, and no deleterious substances are to be found among those dissolved, the removal of the solids in solution is not necessary. Where, however, the presence of a specially large amount, or of harmful bodies, is known, recourse is had to the process of distillation in order to get rid of the solids. In this process, advantage is taken of the observation already made - that the solids are not easily vaporisable and remain in the vessel in which water is boiled.

Experiment 121. Half fill the body of a tubulated (i.e. stoppered) retort with tap-water. Arrange the retort upon a stand with its neck dipping into a small flask, which floats on the surface of water in a large earthenware trough, as indicated in Fig. 85. Heat the water in the retort to boiling-point by means of a small Bunsen flame. Some of the steam produced will condense in the neck of the retort and in the flask. 
When about 2 c.c. have been collected in the latter, remove the burner, and rinse out the flask with this first portion of the distillate. Throw away the rinsings. Replace the flask in position and heat the retort once more. When about half the water in the retort has boiled away discontinue the heating and preserve the contents of the flask for further investigation.

Taste the distilled water after it has become cold and compare its taste with that of some of the tapwater. It will be found to be very insipid in comparison with the latter. Evaporate six drops on a watchglass by the method of Experiment 119. If the

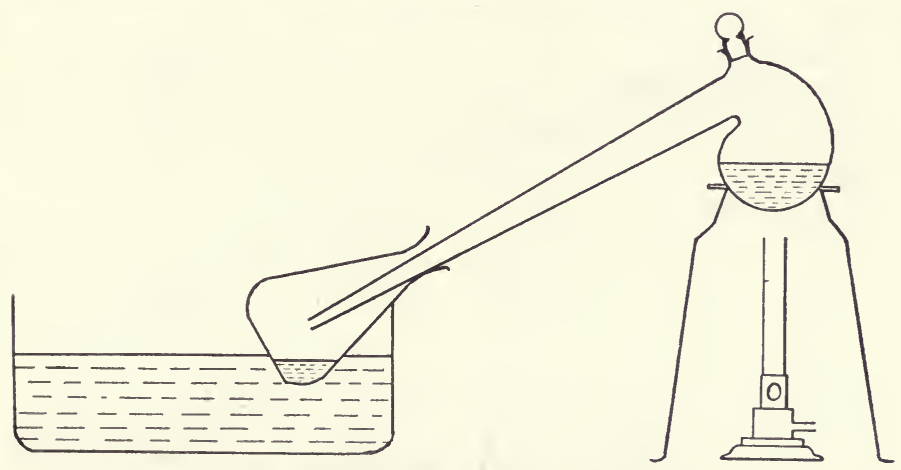

Fig. 85 .

distillation has been carefully carried out, the residue will be scarcely appreciable in amount. (Total absence of residue must not be looked for, since water at the boiling-point attacks glass to a slight extent, the attacked portion dissolving in the water, and being left behind when the water is evaporated to dryness.) Examine the appearance of the water left in the retort and that of the bottom and sides of the vessel which have been in contact with the boiling water. The 
remaining liquid will in general be somewhat turbid and the bottom of the retort will be found to be covered with a thin deposit which is not readily removed by shaking up the water in the retort. The significance of these observations will be considered in Part. II, when dealing with the question of the "hardness" of water.

185. In the experiment just described, no special precautions were taken to ensure the complete condensation of the steam produced by the boiling of the water. As we were only concerned with getting a sample of water for examination, the loss of part of

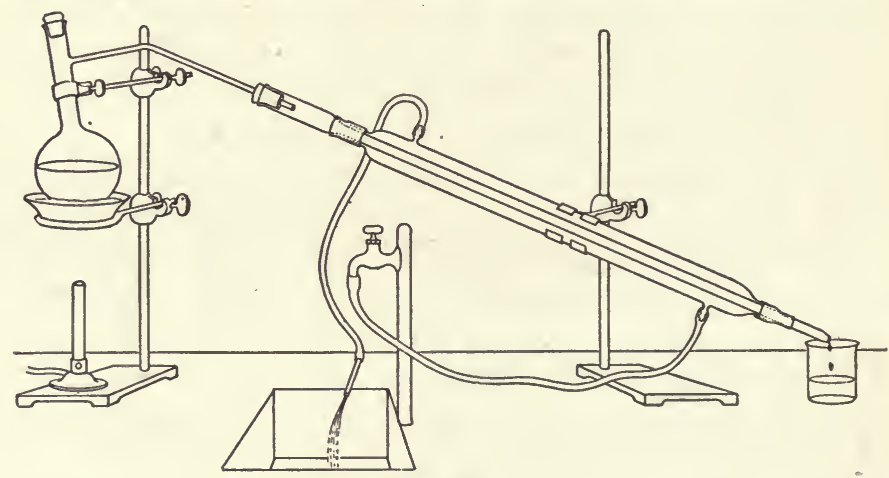

Fig. 86.

the distillate was of no special moment. Where distillation is carried on in order to procure a supply of water in fit condition for drinking from water from a tainted source, or from sea-water, the condensation of the entire quantity of steam becomes important.

Experiment 122. Dissolve about $3 \mathrm{~g}$. of common salt in 100 c.c. of water. This will represent sea-water, if a supply of this be not available. Pour this solution into the flask represented in Fig. 86. See that the 
stopper fits the neck of the flask closely. Pass the end of the side tube of the flask through the hole in the cork fitted into the upper end of the piece of apparatus shown, which is called a Liebig condenser. Arrange the remainder of the apparatus as indicated. The flask is placed upon a sand-bath-a shallow tin dish on which is spread a thin layer of dry sand. Note that the rubber tube from the tap is connected to the lower of the two small tubes projecting from the jacket of the condenser, while the tube leading from the upper end is placed near a sink. Fill the condenser with water by gently turning on the tap, and arrange the flow of water through the jacket so that a steady stream of water issues from the end of the tube placed in the sink. Boil the solution in the flask and, after rinsing the receiver with the earliest portion of the distillate, collect until about half the liquid has been turned into vapour. If the rate of flow of the condensing water is regulated properly, no escape of uncondensed steam should be observed at the lower opening of the condenser.

Test the degree of purification effected by evaporation of samples of the original solution and of the distillate in the manner already described. Similarly. treat a sample of the liquid left in the still and note the amount of solid residue as compared with that left by the original solution.

186. On the large scale, water is boiled in large metal vessels-frequently made of copper-and the steam passes through a long coil of tin pipe immersed in a large vessel through which cold water is circulated. A form of this apparatus is illustrated in Fig. 87. Tin is chosen as the material for the construction of the condenșing worm, since, among the metals in common 
use, it is least affected by the action of steam and pure water.

The nature of the substances to be found dissolved in a sample of ordinary water will receive full treatment in the section of Part II dealing with the domestic uses of. water.

187. On evaporating the various samples of water as above, the residues will have been observed as consisting of masses of indeterminate shape, the separate particles of which each is made up being indistinguishable from one another. By stopping the heating before dryness is reached it is possible to obtain larger

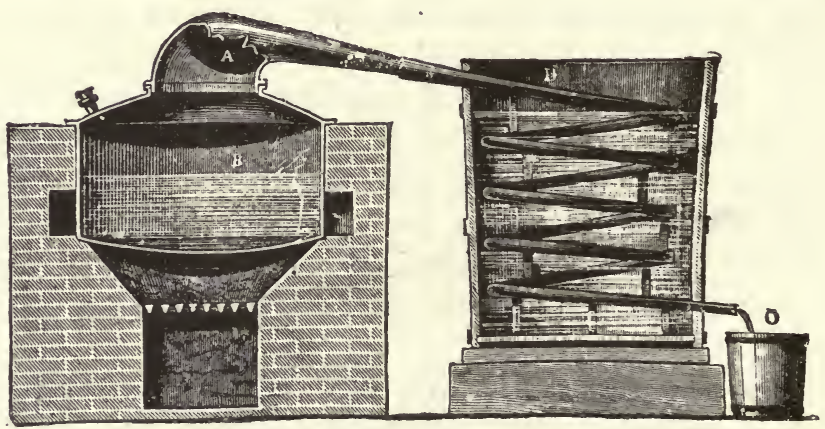

Fig. 87.

particles in many cases, the shapes of these being definite, and the substance previously held in solution may frequently be recognised by examination of the form of the separate particles deposited on partial evaporation.

Those substances which are soluble in water are not all dissolved to the same extent by equal quantities of water. Further, the degree of solubility of any given substance is found to depend upon the temperature of the solvent-in some cases to a very considerable extent. 
Some experiments will next be described in illustration of the three points just mentioned.

ExPERIMENT 123: Into a small beaker, half filled with distilled water, drop a little powdered alum. Stir the water and alum well with a glass rod. Should all the alum be caused to dissolve by this method, add a little more, and keep the mixture well stirred for several minutes after no more of the alum seems to disappear. Drop six drops of the solution thus obtained on a watch-glass and evaporate to dryness. Put the beaker on a piece of wire gauze on a tripod stand and heat it with a good Bunsen flame. Drop in small quantities of alum from time to time until no more will dissolve, even though the liquid be boiled vigorously for a full minute. Evaporate six drops of this solution as before. From the comparison of the amounts of residue obtained in the two cases, and by noting roughly the amounts of alum dropped into the cold and the hot water respectively, it will be seen that alum is very much more soluble in hot water than in cold. Allow the hot solution to cool. As it cools, small -particles of alum begin to appear in the liquid, chiefly at the bottom of the beaker. By the time the solution has reached the temperature of the surrounding air, most of that previously dissolved will have thus separated out. Examination of the alum at the bottom of the vessel may show that the particles are of regular shape, but usually they are too small to show this, when examined by the naked eye. To obtain larger particles the solution should be cooled very slowly or a single small particle should be immersed at the end of a thread attached to a glass rod, the latter resting on the mouth of a beaker containing the remainder of the solution poured off from the solid which has been 
deposited. Allow evaporation to take place slowly at the room temperature for a considerable time and the small piece of alum will be observed to gradually increase in size. After some days its shape should be easily recognisable, and is usually somewhat like that shown on the left hand in Fig. 88. Such regularlyshaped lumps are termed "crystals" and the process by which they are produced is termed "crystallisation".

188. When a liquid has dissolved as much of a given solid as possible at a given temperature, the solution is called a "saturated" solution. By raising the temperature of the liquid it may in general be caused to dissolve more solid, and unless more is thus
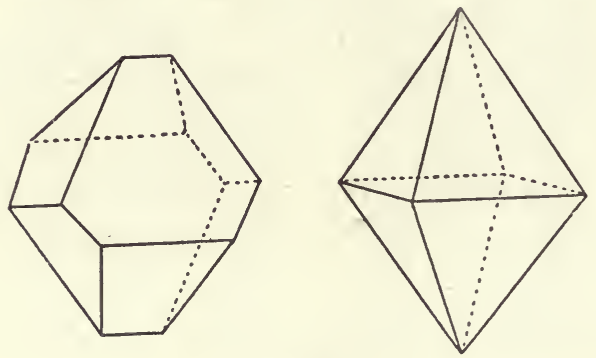

Fig. 88.

dissolved, the solution becomes unsaturated as the result of the rise in temperature. On the other hand, cooling the liquid causes it to become supersaturated and the quantity of solid in excess of that necessary to produce a saturated solution at the new temperature separates out. It occasionally happens that the beginning of crystallisation is delayed in the case of clear solutions in clean vessels, and it is thus feasible to prepare a solution containing a greater quantity of dissolved substance than would normally be the case. 
Experiment 124. Thoroughly clean a flask of about 500 c.c. capacity by rinsing it with tap-water several times, and following this by two or three rinsings with distilled water. Measure out 200 c.c. of distilled water into the clean flask. Weigh out $100 \mathrm{~g}$. of crystallised sodium sulphate-Glauber's salt-and place the crystals in the flask. Stand the flask in a water-bath, heating the latter gently so that the temperature of the water remains practically constant at $33^{\circ} \mathrm{C}$. Shake the flask and its contents frequently. When all the crystals have dissolved examine the liquid produced. This should be perfectly clear. Any crystals undissolved or any particles of other substances floating in the liquid may be removed by filtering (see Experiment 126), the liquid filtrate being received in another thoroughly cleaned flask standing in the water in the water-bath. When a clear solution has been obtained remove the flask from the water-bath, closing the mouth lightly with a wad of cotton-wool. Let the flask cool slowly down to the temperature of the laboratory. It now contains a much larger quantity of dissolved sulphate than is required to form a saturated solution at ordinary temperatures, but, if the experiment, has been carried out with sufficient care, no separation of crystals will occur. Wash the bulb of a thermometer with tap and distilled water and insert it in the sodium sulphate solution. Note the temperature recorded by the instrument. Should its introduction not have been followed by the immediate formation of crystals, as is sometimes the case, drop a very small piece of sodium sulphate into the solution. This will start the crystallisation, and the whole mass of liquid will apparently become solid in a few seconds. Read the thermometer as soon as this has happened.

H. D. S. 


\section{Exercise for Student.}

How do you explain the change of temperature noted as a result of the rapid crystallisation of the sodium sulphate? Test your explanation by finding whether any change of temperature occurs when $100 \mathrm{~g}$. of the sulphate are dissolved in 200 c.c. of water.

189. EXPERIMENT 125. Grind up some potassium chlorate with a clean pestle and mortar. Half fill a beaker with distilled water and place it in a water-bath partly filled with water. Drop some of the chlorate into the water and, by shaking and stirring, try to dissolve it. Should all this first portion dissolve, add more and shake and stir again, repeating the addition till part of the solid refuses to dissolve, in spite of agitation for several minutes. Allow the undissolved portion to settle. Weigh a clean porcelain dish and pipette 20 c.c. of the clear solution into this dish.

Weigh the dish and solution. Evaporate the liquid to dryness over a water-bath. When dry, cool and weigh the dish and contained residue. The difference between the weight of the dish and residue and that of the dish alone is the weight of solid dissolved in the weight of solution given by the subtraction of the weight of the empty dish from that of the dish and solution. By gently heating the water-bath, a second determination of the weight of dissolved substance in a known weight of solution may be made at a higher temperature, say $30^{\circ} \mathrm{C}$. Other determinations at different temperatures may be performed, care being taken to agitate

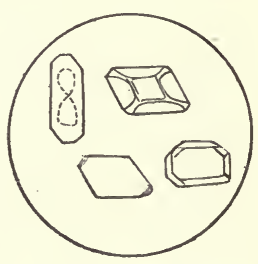

Fig. 89 . the solid thoroughly all the time it is being dissolved. By allowing one of the solutions made at a higher - temperature to evaporate slowly, beautiful crystals 
of similar shapes to those illustrated in Fig. 89 may be obtained.

190. Matter in suspension in water, i.e. not dissolved, but merely floating about, may be removed by filtration, a much simpler process than that of distillation. Porous materials, such as blotting-paper, filterpaper, unglazed porcelain, and layers of sand, are among the filtering materials in common use. To show the applicability of the process to a simple case, try
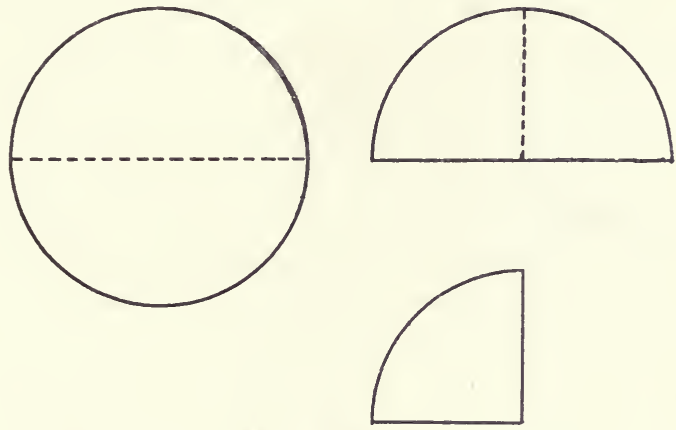

Fig. 90.

Experiment 126. Weigh out $10 \mathrm{~g}$. of whiting and $10 \mathrm{~g}$. of common salt. Thoroughly mix these by grinding them together in a mortar. Test the efficiency of your mixing as follows: Weigh out $5 \mathrm{~g}$. of the mixture and put this weight into a beaker with about 100 c.c. of distilled water. Heat this till the water reaches boiling-point. While the heating is proceeding fold a circular sheet of filter-paper exactly in half, fold the doubled paper again into half, and then open out the quarter-circle of paper into the shape of a cone by inserting the finger so that three thicknesses of the paper are on one side of the finger and one thickness on the other. The stages of folding are illustrated in 
Fig. 90. The filter-paper thus prepared will exactly fit a funnel, if the sloping sides of the latter make an angle of $60^{\circ}$ with each other at all points. Common funnels are not usually so well made as this, hence, to make the conical filter fit the funnel, it is frequently necessary to make the second fold in such a way that the semi-circle is not exactly bisected. The paper is then opened out so that a cone with broader apex or otherwise, as required, may be obtained, according to the bluntness or sharpness of the angle of the funnel. Wet the filter with a few drops of distilled water and press it firmly against the interior of the funnel. Pour the warm liquid from the beaker through the funnel, supporting the latter on the ring of a retort stand, and allowing the liquid which passes through - the filtrateto run into a clean beaker placed underneath the funnel. The filtrate should be quite clear. When almost all the water has been poured through the filter add about 20 c.c. of distilled water to the residue left in the beaker, heat it to boiling and again filter. Repeat the addition of water and filtering at least twice. Test whether any soluble matter is left in the filtrate passing through by evaporating a drop to dryness on a watch-glass and observing whether any residue remains. When the production of no appreciable quantity of residue as a result of evaporation occurs, complete the draining, allowing the liquid adhering to the filter-paper to drain into the beaker, and then transfer the contents of the latter to a weighed porcelain dish. This transference may be done in two or more operations, if the quantity of filtrate is more than enough to fill the dish. Wash out the beaker with small quantities of distilled water and add the washings to the solution in the porcelain dish. Evaporate the contents of the dish 
to dryness and, when cool, weigh the dish again. The gain in weight should be $2.5 \mathrm{~g}$., if the mixing was thoroughly done.

A check on this result may be performed if the filter-paper before use be weighed after drying for a quarter of an hour in a steam-oven. In this case, all the undissolved portion must be washed out from the beaker in which the dissolving took place into the filter, washed several times by pouring small quantities of distilled water through the paper, and the filter and its contents weighed after drying in a steam-oven for a quarter of an hour. To make sure that all water has been removed, the paper should be put back into the oven for five minutes and again weighed. Loss in weight means that more water has been evaporated, and further drying should be resorted to until the results of two consecutive weighings agree. The weight of dry filter and residue, less that of the filter, is that of the quantity of undissolved matter.

191. Should the substance held in suspension consist of large or heavy particles, an almost complete separation of the liquid and the suspended substance may often be made by "decantation". The vessel containing the liquid is allowed to stand in a quiet place for some time so that the suspended matter settles at the bottom of the vessel. The liquid is then poured, care being taken to agitate it as little as possible, into another vessel. A glass rod held against the edge of the pouring vessel at the point where the liquid is escaping serves as a guide for the direction of the liquid into the lower vessel and prevents it from running back down the side of the pouring vessel.

192. To discover whether a given substance is or is not soluble in water is a simple matter. 
Experiment 127. Suppose that we wish to find out if marble is at all soluble in water. Put about $1 \mathrm{~g}$. of marble in a beaker containing at least 100 c.c. of distilled water. Heat the water to boiling-point and continue the heating for about five minutes after the water starts boiling. Filter, if suspended particles render this operation necessary, and put six drops of the filtrate, or, if filtration be not resorted to, of the clear liquid above the marble, into a watch-glass and evaporate to dryness. Similarly treat six drops of the distilled water used in making the solution and compare the quantities of residue left in the two cases. Should no difference in amount be perceptible, the marble is insoluble, and vice versa.

Test the solubility of each of the following substances, and group them according as your results indicate them to be readily soluble, slightly soluble, or insoluble :

Plaster of Paris, red lead, copper oxide, slaked lime, bronze (a penny will do for this, if cleaned before use), powdered glass, calcium chloride, mustard, and cocoa.

193. Liquids resemble gases and solids in exhibiting varying degrees of solubility in water. Some, such as alcohol, dissolve in all proportions and are said to be perfectly miscible with water; others, of which ether is an example, will dissolve to a limited extent, and are termed partially miscible ; while many liquids, especially those of a fatty nature, such as paraffin and olive oils, are almost insoluble in water and are said to be immiscible with that liquid.

Expertment 128. Into each of three 200 c.c. graduated cylinders, provided with close-fitting stoppers, pour 100 c.c. of water. To the water in the first add 20 c.c. of alcohol (spirits of wine), to that in the second 
20 c.c. of ether, and into the third pour 20 c.c. of olive oil. Securely stopper each vessel and shake them well. Allow each, after shaking, to remain undisturbed for some time. Note whether any separation of the liquid mixture into two layers occurs. Read off the number of e.c. in the upper layer, should this happen. Subtracting the values thus obtained from 20 c.c.- - the volume originally used-will provide a rough idea of the amount of liquid dissolved by 100 c.c. of water in each case.

\section{QUESTIONS}

1. Make a list of all the properties of water dealt with in Chapters I to XVI.

2. What methods would you employ to obtain.(a) a sample of clear water; $(b)$ a sample of pure water, from some muddy riverwater? How would you make certain that the latter sample was really pure water ?

3. One kilogram of water from the Irish Sea was shown by analysis to contain $26.43 \mathrm{~g}$. of common salt. The specific gravity of the water at $15^{\circ}$ was 1.02484 . What weight of salt would be contained in the residue left on evaporating 100 litres of the water ?

4. $20 \mathrm{~g}$. of a substance were shaken for 30 minutes with $100 \mathrm{~g}$. of distilled water. The resulting liquid was filtered and $20 \mathrm{~g}$. of the filtrate on evaporation to dryness left a residue weighing $2 \cdot 74 \mathrm{~g}$. Find the percentage solubility of the substance, i.e. the weight of it which will dissolve in $100 \mathrm{~g}$. of water.

5. Suggest possible reasons for the direction in the text that the first portion of each distillate should not be preserved.

6. Devise a method of finding out whether sodium thiosulphate ("hypo") will form a supersaturated solution.

7. Describe in full the method you would adopt in order to obtain large crystals of washing-soda. 


\section{CHAPTER XVII}

\section{THE CHEMICAL RELATIONS OF WATER}

194. In Chapter XIII oxygen was shown to be present in air to the extent of some $20 \%$ of that substance. This element is also contained in water, eight-ninths of the latter by weight consisting of oxygen. The remainder of the water consists of hydrogen, an element known in the free state as a colourless gas.

Experiment 129. Drop a very small piece of sodium (see Experiment 117) on the surface of a large quantity of water contained in an earthenware trough. The sodium floats, moving about on the top of the water as a small molten ball, and making a hissing noise as it does so. The ball slowly gets smaller, and finally the sodium entirely disappears. We have already discovered that sodium combines with oxygen with great vigour. During its wanderings on the water surface it has been combining with the oxygen of the water and part of the hydrogen, the remainder of the latter escaping into the air. Sodium hydroxide is formed as a result of the combination, and its presence in solution may be shown by dipping a red litmus paper into the water after all action has ceased.

The evolution of the hydrogen may be rendered more readily apparent if the free movement of the sodium be prevented by dropping it on a sheet of filter-paper floating on the water. The heat produced by the chemical action is thus concentrated in one place, and the temperature produced is sufficient to 
ignite the hydrogen, which burns above the sodium - with a vivid yellow flame. (The colour is due to a small quantity of sodium vapour being burnt together with the hydrogen, and is not that of the flame of burning hydrogen, as will be seen in later experiments.) When the burning has finished, a small glassy ball of molten sodium hydroxide may be seen floating on the water. This is not in actual contact with the water at first, but, as it cools, a point is reached at which contact occurs, when the hot substance reacts with the water with some violence. Splashes of hot solution of the hydroxide may be thrown to a short distance, hence the observer should not stand too close to the trough during this stage of the experiment. The student will now realise the need for the precautions taken in Experiment 117 with regard to avoiding the contact of the sodium there used with wet bodies.

Equation :

Sodium + hydrogen hydroxide $=$ hydrogen + sodium hydroxide (water) (caustic soda)

195. A few other metals, e.g. potassium and calcium, also possess the power of turning out part of the hydrogen from cold water. Others, such as magnesium and iron, will deprive steam of its oxygen, while. a third group, of which copper and silver are examples, do not act upon either water or steam.

Experiment 130. Fig. 91 represents a long tube, which may be of iron or porcelain, the interior of which is loosely filled with clean iron filings, or with the common small iron tacks, or with small clean screws. The large flask contains water and is supported upon a sand-bath over a Fletcher burner. The long tube rests in an iron trough placed above a furnace consisting of 
a number of powerful Bunsen burners. (In the diagram the tube is shown above the iron trough, but should lie in contact with it while in use.) A bent glass tube connects the flask with the long tube, and a glass delivery tube leads into the water in an earthenware trough. Having fitted up the apparatus as figured and described, light the burners under the flask and tube, the flask being temporarily disconnected from the tube. When the bed of the furnace is at a dull red heat, and the water has reached boiling-point, attach the flask once more and allow the steam to pass through the tube. After allowing an interval of about two minutes for the

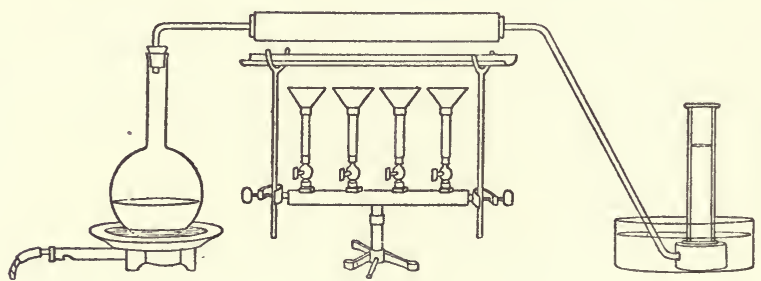

Fig. 91.

escape of most of the air from the apparatus, place an inverted gas-jar full of water over the hole in the beehive cell. Bubbles of gas rise to the top of the jar, which is rapidly filled. Collect another jar in the same way. To show that hydrogen is contained in the jars, put a lighted taper to the mouth of one, held in an inverted position, and the gas will burn at the mouth of the jar. Place the other jar on the bench, remove the glass plate, and leave the jar with its mouth open and directed upwards, for 30 seconds. A lighted taper then plunged into the jar will not cause any sign of ignition of hydrogen to be noted. As we shall show 
later, the gas is much less dense than air and has entirely escaped from the jar.

If the iron used was quite clean and bright at the beginning of the experiment, it may be examined after the tube has cooled, when it will be observed that it is now covered with a film of a blackish substance. This is an oxide of iron called "triferric tetroxide", or magnetic oxide of iron.

Iron + hydrogen oxide $=$ triferric tetroxide + hydrogen.

196. Hydrogen is less chemically active than oxygen and no simple and convenient method is available whereby the hydrogen of water may be readily removed, leaving the oxygen in the free state. To obtain a supply of oxygen from water, recourse may be had to a method whereby electrical energy is transformed into chemical energy with the final result that water is separated into its two elements.

Experiment 131. The apparatus shown in the figure is known as Hofmann's voltameter. The two side tubes are filled with water, to which a little sulphuric acid has been added, by pouring this liquid into the bulbed reservoir, the taps at the top of each side tube being left open while this is done. When enough liquid has been poured in to fill the tubes exactly, the taps are closed. The wires from a battery of two or more Daniell or Bunsen cells, or, if it be available, from the electric light supply, are then connected to the loops of platinum wire near the bottom of each side tube. (If a supply from the electric mains is used, a lamp resistance should be inserted between the loops and the wall plug, as indicated in the figure.) As soon as the attachment is made, bubbles of gas are to be seen ascending from each of the two platinum plates 
contained within the side tubes. At the same time the liquid rises in the centre tube. Allow the action to go on until one of the tubes is filled with gas. Disconnect the supply of electricity. It will be noted that almost exactly half the other tube is filled with gas. The nature of the gas produced in larger quantity may be ascertained by partly opening the tap at the top of the tube and applying a lighted taper to the jet.

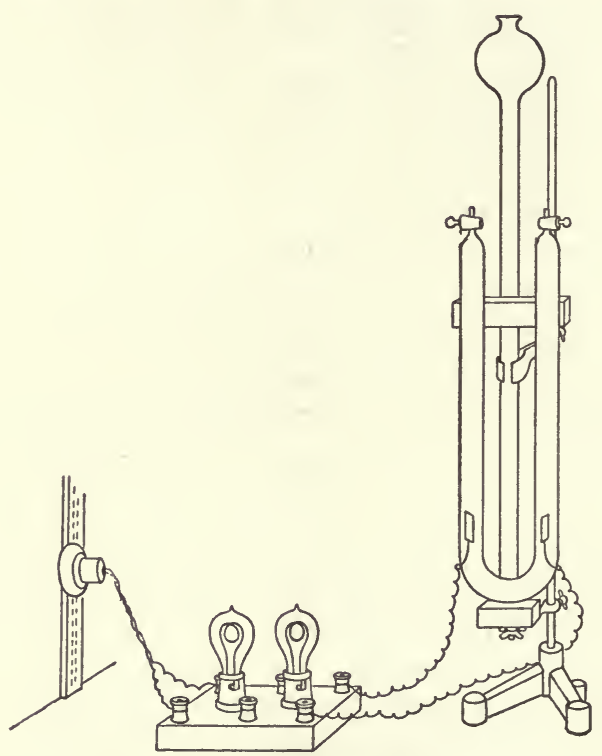

Fig. 92 .

An almost colourless flame- - that of burning hydrogenis seen. Turn off the tap as soon as the liquid reaches the top of the tube. Next partially open the tap of the other tube and hold a glowing splint over the end of the jet. The issuing gas will at once reignite the splint. This we know to be a characteristic property of oxygen. 
The decomposition of the water effected in the above experiment was first discovered by Nicholson and Carlisle in 1801, and their experiment was the forerunner of many other interesting investigations regarding the change of electrical into chemical energy. The operation is known as "electrolysis" and the liquid is called an "electrolyte". The two plates which form the gates, as it were, by which the electric current enters the solution, are termed " electrodes" (Gr. odos, a way).

Incidentally this experiment has shown that two volumes of hydrogen are contained in water for each volume of oxygen. This volume-ratio will be confirmed by the results of later work.

197. In order to obtain a supply of hydrogen for examination of its properties, a different method is generally employed.

Experiment 132. Fit up a flask as shown in Fig. 93. The thistle funnel should be long enough to dip to the bottom of the flask, and the end of the delivery tube should be inserted under a beehive cell standing in water in a trough. The bottom of the flask is covered with a layer of zinc-preferably in the granulated form-and hydrochloric acid, made by diluting

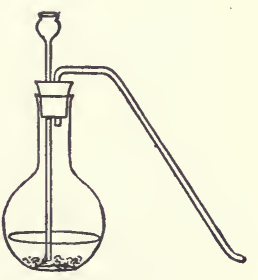

Fig. 93. the strong commercial product with an equal bulk of water, is poured down the funnel. A brisk bubbling of the liquid at once occurs, and gas-bubbles make their appearance at the end of the delivery tube. After waiting a short interval to allow the air in the apparatus to be displaced, collect a test-tube full of the gas issuing from the hole in the top of the beehive cell. When full, close 
the mouth of the tube with the thumb and bring it, mouth downwards, near a flame. Remove the thumb and place the open end of the tube close to the flame. Should a squeaky explosion be heard, collect another sample and again test in the same manner. When the gas burns quietly, a number of gas-jars may be filled for use in the performance of the following tests.

The acid is a solution of a compound of hydrogen with another element, chlorine, and the zinc is able to displace the hydrogen from combination, forming a compound with the chlorine called zinc chloride. The action may be represented thus:

Zinc + hydrogen chloride $=$ zinc chloride + hydrogen .

The zinc chloride remains in solution in the liquid at the bottom of the flask, and the hydrogen, being only slightly soluble in water, is obtained in the free state.

\section{Exercise for Student.}

Devise and carry out a method of obtaining a specimen of zine chloride in the solid state from the solution in the flask.

Show that the gas in the jars corresponds in properties to that collected in Experiment 130 by repeating the tests there suggested. The extreme lightness of the gas may be emphasised by filling a balloon with it.

Experiment 133. The flask in Fig. 94 contains zinc and the acid may be poured as before down the thistle funnel. $T$ is a drying-tube containing small lumps of calcium chloride to prevent the moisture which always escapes with the gas from entering the light collodion balloon $B$. This balloon should not be attached until all the air has been displaced from the flask by hydrogen by allowing the action of the acid 
upon the metal to go on for about two minutes. Before attachment, it should be deflated as far as possible by gentle pressure between the hands. If the gas does not exert sufficient pressure to inflate the balloon properly, cover the mouth of the funnel with the ball of the thumb. The increased pressure thus produced will be generally found to effect the filling without further trouble. When full, release the balloon, which will quickly rise to. the ceiling. After some time, the hydrogen will slowly escape and it will descend and may be preserved for future use.

198. Other metals may replace the zinc in the preparation of hydrogen, and other acids may be used instead of the hydrochloric acid. (Nitric acid is unsuitable, however.) This may be tested by putting

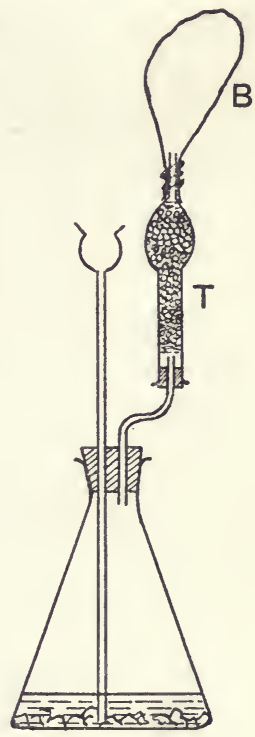

Fig. 94. small quantities of iron, magnesium, and tin at the bottom of separate test-tubes, and pouring various acids, such as sulphuric, hydrochloric, and acetic, upon samples of each of them. In most cases, vigorous effervescence of the liquid indicates the escape of the hydrogen, but where this does not occur, gently warming the tube, or substituting a weaker or stronger sample of acid, will cause the hydrogen to be set free.

Magnesium + hydrogen chloride

$$
=\text { magnesium chloride }+ \text { hydrogen } \text {. }
$$

Iron + hydrogen sulphate

$$
=\text { iron sulphate }+ \text { hydrogen. }
$$




\section{Domestic Science}

Exercise for Student.

Make up the equations for the reactions which occur in the cases of each of the other acids and metals used. Acetic acid is known as hydrogen acetate.

199. Our next investigation will be concerned with the nature of the product formed by the burning of hydrogen. We already know that, in burning, the oxygen of the air combines with the substance burnt to form a new compound, called an oxide. This

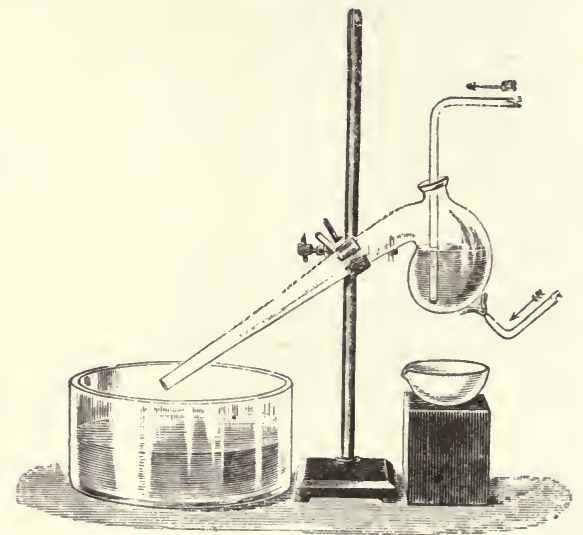

Fig. 95.

is also what happens when hydrogen burns, and the product-hydrogen oxide-is the familiar substance, water.

Experiment 134. Arrange an apparatus for the preparation of hydrogen, drying the gas by means of a calcium chloride tube. To the end of the deliverytube a platinum jet is attached. This is necessary, since the temperature of the flame of burning hydrogen is extremely high, and the edges of a glass jet would 
soon be fused together. Allow the gas to pass through the jet for about a minute and then collect a sample of it by placing a small ignition tube over the jet, the tube being held in an upright position. The hydrogen rises to the top of the tube and the air is gradually displaced downward. This method of collecting a light gas is called "collection by downward displacement of air". When the tube has been in position for several seconds, test the freedom of the gas collected from admixture with air by removing it and lighting it. If it burns quietly, the gas issuing from the jet may be safely lit. Place the jet beneath the bottom of a retort through which cold water is continually circulated from the tap. (See Fig. 95.) The water produced at the high temperature of the flame is naturally in the form of steam. The cold surface of the retort causes part of this steam to be condensed as small drops of water on the exterior of the retort. By continued burning, enough water will be thus condensed to form a large drop at the lowest point of the retort and this may be caught, when it drops, in a clean porcelain dish. To obtain enough water by this means for the performance of several tests is a lengthy process. The simplest plan is to collect about 10 drops in the dish and to apply a test which is applicable to water alone. Dried copper sulphate is a powder of a white or faintly bluish-white colour. Drop a small quantity of this substance into the liquid in the dish. The production of an immediate blue coloration is sufficient evidence that water is contained in the liquid formed by the combustion of the hydrogen. To prove that it is pure water requires further tests to be done.

Hydrogen + oxygen $=$ hydrogen oxide.

H. D. S. 
200. When preparing hydrogen in Experiment 134, the gas was not collected in quantity until a small sample burnt without explosion. The occurrence of an explosion showed that the gas collected was a mixture of hydrogen and air, and not pure, nor nearly pure, hydrogen. The explosion caused by the ignition of this mixture is due to the hydrogen in the test-tube all combining at practically the same instant with the oxygen contained in the air mixed with it. As already mentioned, the combination of these two gases is attended by the liberation of a large quantity of heat, and the enormous expansion caused by the high temperature produced by the heat liberated makes the gases within the test-tube rush out with great violence, and some of the energy generated becomes apparent to our sense of hearing by being transformed into that form of energy which we call sound. The vigorous nature of the explosion may be shown, with perfect safety if the directions are faithfully carried out, by performing

Experiment 135. Drop a few pieces of granulated zinc into a sodawater bottle of the shape shown in Fig. 96. Fit the bottle with a cork through the centre of which a hole of about $\frac{1}{4}$ " diameter has been bored. Remove the cork, pour in a little hydrochloric acid, and replace the cork firmly in the neck of the bottle. Hold the bottle with a duster wrapped around the hand, and bring the opening in the cork close

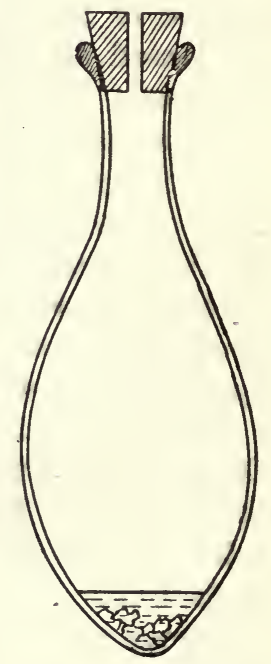

Fig. 96. 
to the edge of a Bunsen flame. See that the mouth of the bottle is pointing towards a wall, and not in the direction of any person in the neighbourhood. When the mixture of gases within the bottle reaches the composition required for explosion, this will occur, with the result that the cork will be ejected to a considerable distance, and the flame of the Bunsen burner will be extinguished.

A mixture of hydrogen and oxygen affords a sharper and more forcible explosion than the above. In this case it is best to use one of the thick-walled glass tubes sold for the purpose, and to dispense with the cork.

201. A simple chemical test was given for the detection of the presence of water in the liquid formed by the combination of hydrogen and oxygen. To prove that a sample thus produced consists entirely of pure water, the following points should be observed :

The liquid must be colourless, tasteless, and odourless. It must leave no residue on evaporation to dryness. It must be of unit specific gravity. Its freezing-point must be $0^{\circ} \mathrm{C}$. and its boiling-point, under a pressure of $760 \mathrm{~mm}$., must be $100^{\circ} \mathrm{C}$. It must not affect the colour of either red or blue litmus paper. It must turn white copper sulphate blue.

202. A distinctive test, in addition to those above enumerated, is to be found in the peculiar behaviour of water when heated from the freezing-point upward.

Experiment 136. Obtain a piece of lead piping"compo" piping, as it is called by plumbers, will do excellently-about $2 \mathrm{ft}$. long. Seal one end of the piping by hammering the edges together. Coil the 
pipe into the form of a flat spiral, with the open end projecting at right angles as indicated in the figure. Fit a length of glass tubing of somewhat smaller bore than the metal piping to the open end by means of a small cork or a short piece of stout rubber tubing. Cool this piece of apparatus by immersion in a vessel containing melting ice. After it has been in the ice for about five minutes, remove the glass tube and fill the metal tubing with ice-cold water. Replace the glass tube, and by pushing it into the metal tube, cause the water to rise some three or four inches in the glass

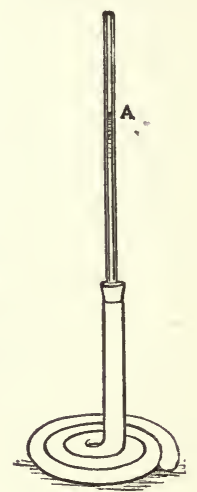

Fig. 97. tube. Leave the leaden tube immersed in the ice for another five minutes, and, at the end of that interval, mark the level of the liquid in the glass tube. Assist the melting of the ice by surrounding the vessel containing it with a.water-bath. The water in the bath should be gently heated and its temperature should not exceed $30^{\circ} \mathrm{C}$. Place a thermometer in the vessel containing the melting ice, and stir from time to time. Should the water in the glass tube fall below the marked position, note the lowest point reached and mark this as before. When all the ice has melted, the temperature of the water formed will gradually rise. At this stage remove the source of heat. As the temperature of the ice-water slowly rises, the water in the glass tube will be seen to sink. This indicates that this water, while its temperature is being raised, is contracting, thus behaving in the reverse manner to that previously observed as common to other substances. The decrease in volume will continue until a temperature of about 
$4^{\circ} \mathrm{C}$. is reached, after which the slow rise of the waterlevel in the glass tube will indicate that the water above this temperature is behaving in the normal fashion. From this point, the expansion is continuous until the water begins to boil.

This temperature, $4^{\circ} \mathrm{C}$., is known as the temperature of the "maximum density of water". The anomalous behaviour of water at $0^{\circ}$, when heated, is, of course, paralleled by the reverse effect being produced when water at $4^{\circ}$ is cooled. This peculiarity is of great importance as a natural agency in the preservation of animal life in shallow waters in winter, and in maintaining a temperate climate over portions of the globe which would otherwise be regions with a very low mean annual temperature. When water in a lake is cooled by contact with the cold air above it, the surface water, contracting, becomes denser, and convection currents are set up whereby the warmer water is constantly moving towards the surface and the cooled water is descending. When the temperature of the water at the surface has fallen to $4^{\circ}$, further cooling causes expansion and it no longer sinks, since its density is now lessening. Continued exposure to cold causes the surface water to lose heat until its temperature has reached freezing-point. 'Ice crystals then begin to form slowly at the top of the water, and float on account of their density being less than that of water. A continuous sheet of ice forms as a result of the agglomeration of the crystals. This forms a protective coating for the water beneath, ice being a bad conductor of heat. Thus the thickening of the ice-sheet proceeds slowly, and the water, except that in immediate contact with the ice, is at the uniform temperature of $4^{\circ} \mathrm{C}$. This is warm enough 
to permit fish to remain alive, and, unless the cold weather be excessively prolonged in duration, the water in lakes and ponds, except where they are extremely shallow, does not all become solid.

\section{Exercise for Student.}

From what has been already stated, investigate what would happen in very cold weather, if water did not possess a point of maximum density.

Thus, if a liquid be pure water, it must, in addition to complying with the requirements of the tests given in the list above, exhibit the property of abnormal expansion between the temperatures $0^{\circ}$ and $4^{\circ}$.

203. The question of the exact composition of water has been the subject of many careful investigations. To determine the relation by weight between the quantities of hydrogen and oxygen present in a given amount of water, we may synthesise that compound as described in the next experiment.

Experiment 137. A steady gentle stream of hydrogen is required in this experiment. This may best be obtained by the electrolysis of water as previously described. An alternative method, in which the hydrogen produced is of a lesser degree of purity, is afforded by the use of a "Kipp's generator". This is illustrated in Fig. 98. The middle bulb of the apparatus should be filled about one-third full with scraps of the purest zinc foil obtainable. Open the tap at the side of this bulb and pour down the opening of the topmost bulb a mixture of pure hydrochloric acid and water in equal proportions. When the lowest bulb is full of the liquid, close the tap and then pour in more acid until the top bulb is about one-third full. The apparatus is now ready for use. Open the 
tap. The pressure of the liquid in the centre tube and in the top bulb forces the air out of the middle bulb through the tap. The acid from the bottom bulb rises through the neck between that and the middle bulb and thus comes in contact with the zinc. Hydrogen is immediately evolved and issues from the tube connected with the tap. Allow the action to continue

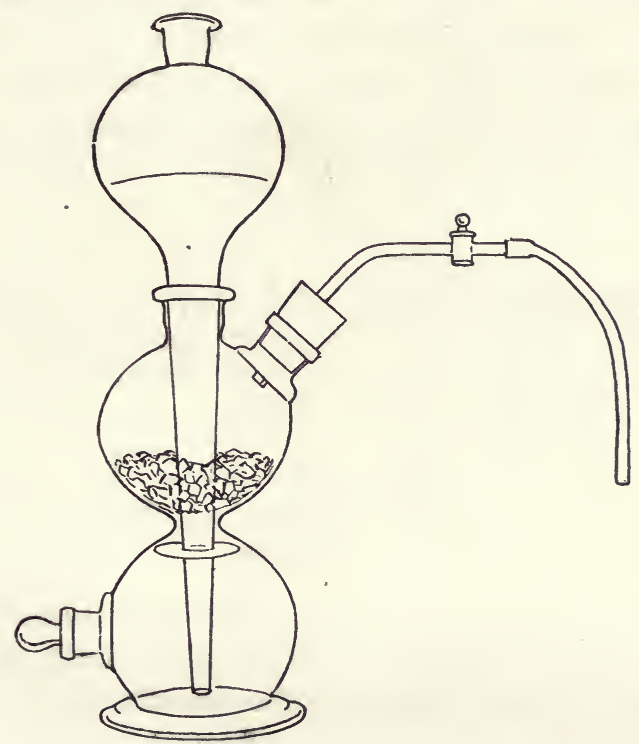

Fig. 98.

for a short time in order to expel as much air as possible from the middle bulb. Close the tap. Hydrogen is still generated by the action of the acid remaining in contact with the zinc, but can no longer escape. Hence the pressure of the gas in the middle bulb increases and, as a result, the acid is forced out of the middle bulb and returns to that at the top, the action ceasing 
when no more acid is left on the zinc. A further supply of the gas may be at once obtained by simply opening the tap.

To determine the composition of water by weight, the delivery tube of the hydrogen generator is connected to the tube marked with an arrow in Fig. 99. The first drying-tube contains a small quantity of the strongest sulphuric acid, while the second is nearly filled with granulated calcium chloride. The bulbed tube and its content of copper oxide is weighed carefully before the commencement of the experiment. Both tube and copper oxide should be dried in a hot air oven

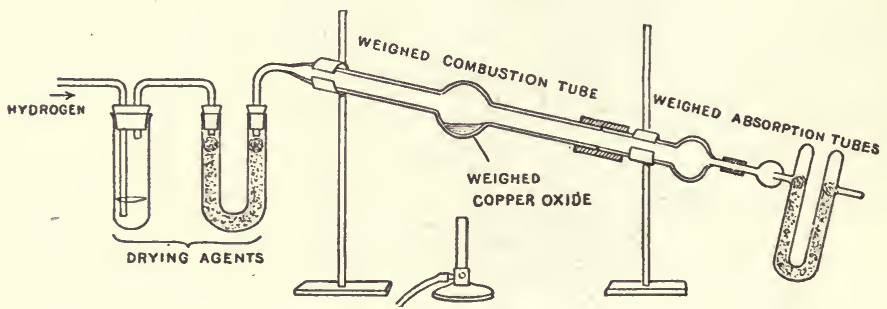

Fig. 99.

for 15 minutes at a temperature of about $110^{\circ} \mathrm{C}$. before being weighed. The smaller bulb and the attached calcium chloride tube are for the collection and absorption of the water formed, and should be weighed before starting. When the apparatus has been set up, it should be tested to see whether it is gas-tight. Attach a glass tube to the end of the last absorbent tube, and let the end of this tube dip beneath the surface of water in a beaker to a depth of three inches. Allow a little hydrogen to pass through the apparatus: This should cause immediate bubbling in the water and, when the stream of hydrogen is 
discontinued, the water should not rise in the glass tube. Having secured a satisfactory result, remove the glass tube. Pass hydrogen through the apparatus slowly so that the bubbles rise in the sulphuric acid at the rate of about two per second. Collect a sample of the gas issuing from the calcium chloride tube and test its character by lighting it. As soon as the sample burns quietly, light the Bunsen burner underneath the hard glass bulb. The oxide should be raised to a dull red heat. Continue the passage of the gas until the oxide has assumed a brownish-red tint and an appreciable quantity of water has collected in the smaller bulb. Turn out the burner, and let the apparatus cool. In exact work, a stream of dry air is passed through the apparatus by disconnecting the hydrogen generator, and replacing it by a gas-holder containing air, the air being sent through for two or three minutes at about the same rate as in the case of the hydrogen.

\section{Exercise for Student.}

Why is this passage of dry air necessary for an exact determination to be effected?

When quite cool, the portions previously weighed are again weighed separately. The gain in weight of the absorbent portion is that of the water formed as the result of the action of the hydrogen on the hot oxide. The loss of weight of the combustion tube and oxide is the weight of oxygen which combined with the hydrogen to form this water. The weight of the hydrogen may be obtained by finding the difference between the weight of water formed and the weight of oxygen contained in it.

The value of the ratio $\frac{\text { weight of oxygen }}{\text { weight of hydrogen }}$ should be 
calculated and should approximately be 8 . As the mean result of the best determinations, the value 7.94 is now generally accepted. The student must not expect to obtain a very close approximation to this value at the first attempt, but a fair degree of accuracy may be attained by careful attention to the directions supplied.

Hydrogen + copper oxide = copper + hydrogen oxide.

Exercise for Student.

Having given that the specific gravity of hydrogen is 0.00008989 , and that of oxygen 0.0011054 , find what volume of hydrogen will combine with 1 c.c. of oxygen.

204. It is interesting to note that the oxide of iron produced in Experiment 130 may be once more reduced to the metallic state if a hydrogen generator be substituted for the flask and the jar be replaced by a flask floating on cold water, the end of the delivery tube being inserted into the mouth of the flask. Pass a steady stream of hydrogen from the generator through the tube containing the iron oxide for three minutes. Next heat the tube to dull redness, allowing the gas to pass continuously. In a short time small drops of water will fall into the flask, and, by sufficiently prolonging the experiment, the whole of the oxygen may be removed from the oxide in this way. Hydrogen + triferric tetroxide $=$ iron + hydrogen oxide.

On examining this equation, it will be seen that it is the reverse of that given at the end of Experiment 130. Many other cases are known of similar reactions, which may take place in opposite ways according to the conditions of the experiment. In that just described the steam produced is carried away by that part of the stream of hydrogen which is unaffected and thus 
prevented from again oxidising the iron. In Experiment 130 , the steam was in excess, and the hydrogen. formed was removed from the tube by the pressure of that portion of the steam which escaped without reacting on the iron. Such reactions as this are termed "reversible" reactions, and the equations representing them are written thus:

Iron + hydrogen oxide

$\rightleftarrows$ hydrogen + triferric tetroxide.

The two arrows with their points oppositely directed denote that the reaction will take place in the forward or backward direction according to the conditions under which the action is carried out.

\section{Exercise for Student.}

Try to find other cases of reversible reactions in the work already performed. Write the equations representing any that may be discovered so as to show their reversible nature.

205. The relation between the volumes of hydrogen and oxygen which combine with one another may be determined by means of an instrument known as the $\mathrm{U}$-tube eudiometer. This is shown in the annexed figure. A measured volume of oxygen is introduced into the closed graduated limb, the volume being read off after adjusting the quantity of mercury until it stands at the same level in both limbs. Hydrogen is next introduced, usually in considerable excess of the amount needed to combine with the oxygen

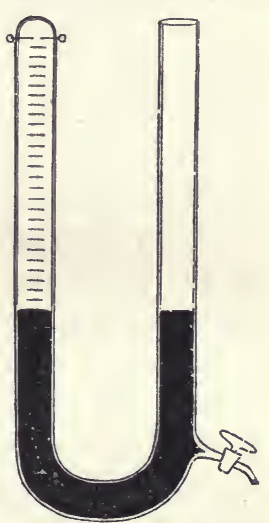

Fig. 100. previously passed in. The combined volume of the two 
gases is then read off as before. A further quantity of mercury is then poured into the open limb so as to fill it within 6 " of the top and this limb is closed with a tightfitting rubber stopper. An electric spark is then caused to pass between the ends of the wires fused into the upper end of the closed limb. This is effected by connecting the outer ends of the wires to the wires from a small induction coil, and momentarily switching on the current. The passage of the spark causes explosive combination to occur, and on removing the stopper from the open limb, a contraction in volume of the gases in the other limb is seen to have taken place. On reading off the enclosed volume after levelling the surfaces of the mercury in the two limbs, the decrease of volume is found to be three times that of the oxygen used. The residual gas, on being tested, may be shown to consist entirely of hydrogen. Two volumes of hydrogen have therefore combined with one volume of oxygen to form steam, and, since the volume of water formed from this steam has an almost inappreciable volume as compared with that of the steam (see Chapter $\mathrm{x}$, par. 114), the decrease in volume of the gases in the closed limb of the eudiometer amounts to thrice the volume of the oxygen.

If we desire to know the relation between the volumes of the gases and that of the steam produced by their combination, the closed limb must be surrounded by a jacket, as in Fig. 101. Through this jacket is passed, during the experiment, the vapour of some liquid which boils at a temperature considerably higher than the boiling-point of water. Amyl alcohol is sometimes used, its boiling-point being $132^{\circ} \mathrm{C}$. A condenser is attached to the jacket in order that the alcohol may be recovered for further use and that its fumes, which 
are injurious when breathed, may not escape into the air. When the above experiment is performed under these new conditions the contraction in volume which occurs after the explosion is found to equal the volume of the oxygen introduced, a further contraction of twice this amount being effected by allowing the apparatus to cool. From these observations it would appear that the volume of steam formed by the combination of two volumes of hydrogen with one of oxygen itself occupies a volume equal to that of the hydrogen.

206. A great many experiments have been performed with the object of finding the

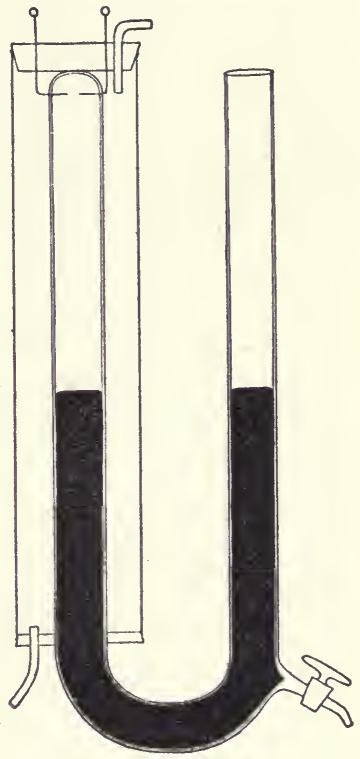

Fig. 101. composition of many other bodies besides water, both by volume and by weight, and one general result of these may be summed up as follows:

In every chemical compound the ratios between the weights of the elements contained in the compound are constant.

This statement is known as the "Law of Constant Composition" and is entirely the outcome of experimental work. No theory of the constitution of matter is involved in any way in the evidence for the validity of the law.

As examples of what is implied by this law we will 
consider the composition of water and of chalk. Pure water, from whatever source obtained, however prepared, and by whomsoever examined, is always found to contain 7.94 parts by weight of oxygen combined with 1 part of hydrogen, provided the examination be carried out with sufficient care. Small variations from these figures may be noted, but none greater than can be accounted as errors of experiment. Again, the percentage composition of pure chalk is always found to be : calcium, $40 \%$; carbon, $12 \%$; oxygen, $48 \%$; or, put in another way, 1 part by weight of carbon is always found in combination with 4 parts of oxygen and 3.33 parts of calcium in every sample of calcium carbonate, of which compound chalk is one of the naturally occurring forms.

207. The composition of water by volume was shown to be of a simple character, two volumes of oxygen combining with one volume of hydrogen to form two volumes of steam. Similar regularity exists between the volumes of other reacting gases. Thus, two volumes of acetylene combine with five volumes of oxygen to form four volumes of carbon dioxide and two volumes of steam. In the form of a general statement, this is known as Gay-Lussac's "Law of Gaseous Combination", and may be put thus:

When gases combine, the volumes of the reacting gases and those of any gaseous products bear simple ratios to one another.

Further reference will be made to both the above laws in later work.

208. In concluding this chapter, the question of the differences between a mixture of substances and a chemical compound will be considered, with special 
reference to air and water as examples of each class respectively.

Air is a mixture because it may be made by simply passing volumes of nitrogen and oxygen, which bear the same relation to one another as do the volumes of these gases found in air, into an empty vessel. The mixture behaves in every way like air. On the other hand, if two volumes of hydrogen and one volume of oxygen be mixed, no water is produced by the mixing, and the mixture behaves neither like water nor steam, since the insertion of a flame causes an explosion.

In the formation of air by the mixing of nitrogen and oxygen, no heat is evolved. The evolution of heat is a common occurrence during chemical combination, and the great quantity of heat produced when the constituent elements of water are caused to unite to form water offers a striking contrast to the absence of heat production in the case of air.

Again, no change of volume follows the admixture of nitrogen and oxygen, while, as we have seen, the steam formed by the combination of hydrogen and oxygen only occupies two-thirds of the combined volumes of the mixed gases. Change of volume is another frequent concomitant of chemical action.

The physical properties of air are the mean of those of the gases nitrogen and oxygen. Thus its specific gravity may be calculated from the ratio by volume in which these two gases occur, when the specific gravities of the gases are known. The specific gravity of steam is not the mean of those of hydrogen and oxygen, as calculated, but is considerably greater. Similarly, the other physical properties of water cannot be foretold from a consideration of those of its constituents. 
The nitrogen and oxygen of air may be separated to some extent by such simple physical operations as diffusion and solution in water (see Chapter xIv, Expt. 105), each gas exercising its own powers quite independently of the other. No such simple means suffice for the separation of the two gases contained in water. A striking example of the independence of the oxygen and nitrogen in this respect is afforded by a manufacturing process for obtaining oxygen from air. Liquid air, produced by exposing air to a low temperature and allowing it to cool itself by rushing through a very small hole under very great pressure, is caused to evaporate. The boiling-point of liquid oxygen is $182.5^{\circ} \mathrm{C}$., while that of the liquid nitrogen is $13^{\circ}$ lower. As a consequence, the latter boils away first, and, by careful manipulation of the conditions of the experiment, the nitrogen may be separated from the oxygen so completely that practically pure oxygen is left. Compare this result with the behaviour of water on boiling.

The small variations noted in the composition of different samples of air are larger than the limits of experiment, hence it does not obey the Law of Constant Composition formulated for chemical compounds.

No less than six distinct compounds of nitrogen and oxygen are known to chemists, and these all obey the same laws as other chemical compounds. This may be taken as indirect evidence in favour of the idea that air is a mixture, and not a compound, of the two gases which are its chief constituents.

Other points of difference exist, but those already mentioned will be sufficient to demonstrate the great differences which exist between a mixture and a chemical compound. 


\section{QUESTIONS}

1. A jar of gas is supplied to you. By what tests would you attempt to find out whether the gas was one of the following: hydrogen, carbon dioxide, oxygen ?

2. A reducing agent may be briefly defined as a substance which readily combines with the oxygen of a compound, leaving another substance with a lesser proportion of oxygen. Give reasons for considering hydrogen to be a reducing agent.

3. Describe any experiments you know which illustrate the energetic nature of the combination of hydrogen and oxygen.

4. Briefly describe the experiments necessary to distinguish clearly between distilled water, river water, and sea water.

5. How may the composition of water by weight be deter-

\section{ERRATUM}

p. 304 , line 14 from top, for $182 \cdot 5^{\circ} \mathrm{C}$ read $-182 \cdot 5^{\circ} \mathrm{C}$.

8. Describe a method of obtaining oxygen from the air by a process not involving chemical change.

9. $15 \cdot 6$ c.c. of oxygen are mixed with $8 \cdot 75$ c.c. of hydrogen in the tube of the eudiometer, and a spark is passed through the mixture. What volume of gas is left, and what is its nature?

10. What alterations of volume occur during the change of a quantity of ice at $-5^{\circ}$ into steam at $105^{\circ}$ ?

11. For what reason is it necessary to dry the hydrogen used in Experiment 134 ?

12. Describe all that is seen when a lighted taper is thrust upward into a dry jar of hydrogen.

13. What is a reversible reaction ? Describe experiments which show that the oxidation of mercury is of this character. Specify clearly the conditions requisite for each phase of the action to take place.

H. D. S. 
The nitrogen and oxygen of air may be separated to some extent by such simple physical operations as diffusion and solution in water (see Chapter xIv, Expt. 105), each gas exercising its own powers quite independently of the other. No such simple means suffice for the separation of the two gases contained in water. A striking example of the independence of the oxygen and nitrogen in this respect is afforded by a manufacturing process for obtaining oxygen from air. Liquid air, produced by exposing air to a low temperature and allowing it to cool itself by rushing through a very small hole under very great pressure, is caused to evaporate. The boiling-point of liquid oxygen is $182.5^{\circ} \mathrm{C}$., while that of the lionid nitrooen is $13^{\circ}$ Inxrar

The small variations noted in the composition of different samples of air are larger than the limits of experiment, hence it does not obey the Law of Constant Composition formulated for chemical compounds.

No less than six distinct compounds of nitrogen and oxygen are known to chemists, and these all obey the same laws as other chemical compounds. This may be taken as indirect evidence in favour of the idea that air is a mixture, and not a compound, of the two gases which are its chief constituents.

Other points of difference exist, but those already mentioned will be sufficient to demonstrate the great differences which exist between a mixture and a chemical compound. 


\section{QUESTIONS}

1. A jar of gas is supplied to you. By what tests would you attempt to find out whether the gas was one of the following: hydrogen, carbon dioxide, oxygen ?

2. A reducing agent may be briefly defined as a substance which readily combines with the oxygen of a compound, leaving another substance with a lesser proportion of oxygen. Give reasons for considering hydrogen to be a reducing agent.

3. Describe any experiments you know which illustrate the energetic nature of the combination of hydrogen and oxygen.

4. Briefly describe the experiments necessary to distinguish clearly between distilled water, river water, and sea water.

5. How may the composition of water by weight be determined ? What weight of water would contain $67.9 \mathrm{~g}$. of oxygen ?

6. Why do lakes and rivers not become frozen solid during long spells of frosty weather?

7. A certain liquid leaves no residue on evaporation to dryness. What further evidence would you require to be presented before deciding whether it is water ?

8. Describe a method of obtaining oxygen from the air by a process not involving chemical change.

9. 15.6 c.c. of oxygen are mixed with 8.75 c.c. of hydrogen in the tube of the eudiometer, and a spark is passed through the mixture. What volume of gas is left, and what is its nature?

10. What alterations of volume occur during the change of a quantity of ice at $-5^{\circ}$ into steam at $105^{\circ}$ ?

11. For what reason is it necessary to dry the hydrogen used in Experiment 134 ?

12. Describe all that is seen when a lighted taper is thrust upward into a dry jar of hydrogen.

13. What is a reversible reaction? Describe experiments which show that the oxidation of mercury is of this character. Specify clearly the conditions requisite for each phase of the action to take place. 
14. How may the volumetric composition of water be determined by (a) a synthetic method; (b) an analytical method ?

15. The weight of 1 c.c. of hydrogen at $0^{\circ} \mathrm{C}$. and $760 \mathrm{~mm}$. bar. is $0.00009 \mathrm{~g}$. What weight of hydrogen would be needed to fill a spherical balloon, 6 metres in diameter, the gas being at a temperature of $12^{\circ} \mathrm{C}$. and under a pressure of $800 \mathrm{~mm}$. bar. ?

16. Devise an experiment in which it is shown by direct weighing that hydrogen is much lighter than air.

17. How may it be demonstrated that water, in cooling from $4^{\circ}$ to $0^{\circ}$, increases in volume?

18. In a determination of the composition of water by the method of Experiment 137, the following observations were made:

Wt. of bulb and oxide before heating _. . $40.63 \mathrm{~g}$.

Wt. of bulb and copper after heating _. . $35.78 \mathrm{~g}$.

Wt. of small bulb and drying-tube before expt. .. 65.34 g.

Wt. of small bulb and drying-tube after expt. $\quad .70 \cdot 81 \mathrm{~g}$.

Find the weight of oxygen combined with $2 \mathrm{~g}$. of hydrogen.

19. Why should the combustion tube and the copper oxide used in Experiment 137 be carefully dried before weighing ?

20. What volume of steam will be formed by the burning of a cubic foot of hydrogen ? What volume of oxygen will be removed from the air as a result of this combustion?

\section{CHAPTER XVIII}

\section{CHEMICAL CLASSIFICATION}

209. The division of substances into the two groups known as elements and compounds has been already dealt with. In this chapter some further subdivisions of these two main classes will receive consideration. 
210. It is usual to divide the elements into metallic and non-metallic sections. To the former belong the majority of the elements, all the common metals being included in this class. The distinction between a metal and a non-metal is not a hard and fast one. In general, a metal possesses the following properties, although every metal does not of necessity exhibit all of them.

(1) Metals are usually of high density.

(2) They possess a characteristic lustre, and their surfaces may be made to take a high polish.

(3) They are ductile, i.e. may be readily drawn into wire.

(4) They are malleable, i.e. may be hammered out into thin sheets without fracture. electricity.

(5) They are good conductors of heat and of

(6) They usually exist at ordinary temperatures in the solid state.

(7) They form oxides which are generally insoluble in water. Those which dissolve produce alkaline solutions.

\section{Exercise for Student.}

Classify the metals already mentioned in the text according as they exhibit or otherwise each of the characteristics given above, as far as your knowledge of the properties of each permits.

To the class of non-metals belong the gases, oxygen, nitrogen and hydrogen already studied, together with the following: carbon, sulphur, chlorine, bromine, iodine, silicon, boron, phosphorus and argon. A few other elements of less common occurrence also belong to the non-metallic group. 
Exercise for Student.

As far as your knowledge serves, show that the substances stated to be non-metallic elements do not agree with the metals in their qualities.

211. The classification of compounds is not so simple a matter as that of elements. Some 200,000 distinct compounds are known to chemists, and the methods of classification adopted are arranged to suit the requirements of various special properties or groups of properties according to the purpose of the classification. It was formerly supposed that one great natural line of demarcation could be drawn between bodies produced by animals and plants as the results of vital processes and those bodies naturally belonging to the world of "dead" things. Thus compounds were classified as "organic" or "inorganic", and this arrangement presents some advantages even at the present day. Organic bodies all contain carbon. The combining powers of this element are somewhat peculiar as compared with those of the remaining elements, a much larger number of compounds containing carbon being known than is the case with any other element. Thus organic chemistry, as it was termed, has now become the chemistry of the carbon compounds. That the intervention of the energies of living matter is a necessary factor in the production of organic substances is, however, now proved to be a fallacious idea. The discussion of the properties and composition of some important organic bodies will be found in Part II of this book.

212. Another method of grouping compounds was based on the number of elements contained in a molecule of each compound. Compounds containing two elements only were called "binary" compounds, and the 
termination of the chemical name of such a compound ends in "-ide". To this class belong the oxides, chlorides, bromides, sulphides, and carbides. When three elements were present in a compound, it was termed a "ternary" compound ; the inclusion of four elements produced a "quaternary" compound, and so on. Those acids which contain oxygen and the metallic compounds derived from these acids, are in general members of the ternary group of compounds. Their characteristic termination is "ate". Thus we have the sulphates, nitrates, phosphates, chlorates, carbonates, and silicates.

213. A very important method of arranging inorganic substances has been indicated in a previous chapter. Certain oxides were termed acidic, while others were said to be basic. The bodies formed by the addition of the elements of water to the acidic oxides are known as acids, while those produced similarly from basic oxides are the bases. The phrase "addition of the elements of water" should be noted. Solution in water is not necessarily intended by this, for we already know that some basic oxides, e.g. iron oxide, do not dissolve in water. This is also the case with a few acidic oxides. Silicon dioxide, or silica, a common form of which is sand, is quite insoluble, yet we are able to obtain it combined with a certain quantity of hydrogen and oxygen, these elements entering into combination in the same ratio by weight as that in which they exist in water, the combination being effected by indirect means. The substance produced is known as hydrogen silicate, or silicic acid, and behaves, in most respects, just like those acids we have already obtained by the solution of a nonmetallic oxide in water (see Chapter xv). 
214. Bases react with acids to form a new series of bodies, termed "salts", water being always simultaneously produced. Thus, if some sodium hydroxide be added to sulphuric acid until the reaction of the resulting liquid to litmus is neutral, and the liquid be evaporated, a white solid, the salt sodium sulphate, is obtained.

Sodium hydroxide + hydrogen sulphate

= sodium sulphate + hydrogen hydroxide.

215. The characteristic properties of these three groups of compounds, viz. acids, bases, and salts, will next be considered.

ExperIment 138. Test hydrochloric, sulphuric, nitric, and acetic acids by performing each of the following simple experiments, using in all cases, except where otherwise mentioned, a mixture of the strong acid with about four volumes of water.

(a) Drop 10 drops of the acid into a beaker half full of water, shake the mixture, and cautiously taste it.

(b) Put a drop of the acid, taken from the bottle on the end of a glass rod, on a blue litmus paper.

(c) Into a test-tube, one-quarter filled with the acid, drop a small piece of granulated zinc. Note all that occurs before the action stops entirely. Warm the tube if necessary.

(d) One-quarter fill a test-tube with acid and drop in about half a gram of powdered copper oxide. Shake well, and hasten the completion of the reaction which occurs by gently heating the tube.

(e) Similarly treat about half a gram of sodium carbonate. In this case heat will not need to be applied.

(f) Put a drop of the strongest form of each acid on a filter-paper and allow it to remain for a quarter 
of an hour. Put a drop of the weaker acid on another paper and, after putting it aside for a day, examine both papers. The effects in each case may be obtained more readily, if required, by gently heating the papers over a small Bunsen flame.

Compare the results obtained for the several acids, and,from your observations, prepare a general statement of the properties of acids.

EXPERIMENT 139. The properties of soluble bases, or alkalis, may be similarly studied. Use solutions of sodium hydroxide, potassium hydroxide, and calcium hydroxide (lime-water) in the performance of each of the following tests :

(a) Taste very weak solutions of each alkali.

(b) Rub a little stronger solution between the fingers.

(c) Put a drop on a red litmus paper.

(d) Invert in a trough of alkali a jar of carbon dioxide, obtained as in Experiment 114, or by the method described in Part II.

(e) Drop strong and weak solutions on filterpaper as in $(f)$ above, and note the action after some time has elapsed.

Formulate a general statement of the properties of alkalis from the results of your observations.

ExPERIMENT 140. Study the action of solutions of the following salts-sodium chloride (common salt), sodium carbonate (washing-soda), and copper sulphate (blue-stone)-when tested as suggested below.

(a) Taste a very weak solution.

(b) Drop a little of the solution upon red and upon blue litmus paper.

(c) Put a crystal of washing-soda in the solution and gently warm. 
(d) Pour a little sodium hydroxide solution into the solution of the salt. the solution.

(e) Pour a small quantity of sulphuric acid into

Tabulate your results and note that the behaviour of salts is less uniform than that of acids or of alkalis.

In general, salts in solution will give a neutral reaction with litmus, but some salts, such as the sodium carbonate in the last experiment, have an alkaline reaction, while others, e.g. copper sulphate, produce the opposite effect. These exceptions are known as " salts with an alkaline reaction" and "salts with an acid reaction " respectively. They must not be confounded with " acid" salts and "basic" salts (see later portion of this chapter).

\section{Exercise for Student.}

Test the reaction of as many salt solutions as you can, classifying them as of neutral, acid, or alkaline reaction, according to the results of your tests.

216. We have previously discussed the suitability of Lavoisier's name for the element oxygen, and it should be noted that all acids formed by the combination of an acidic oxide with the elements of water do contain this element. They are known as the "oxy-acids". Sulphuric, nitric, and carbonic acids fall into this group. Such an acid as hydrochloric acid does not contain oxygen, being formed by dissolving a compound of the elements hydrogen and chlorinehydrogen chloride - in water (see Part II). The water does not combine with the hydrogen chloride, as may be shown by merely boiling the solution. According as this be strong or weak, hydrogen chloride will be evolved, making the strong solution weaker, or water 
will boil away, leaving a stronger solution. The element hydrogen is contained in all acids, however, and it is by the replacement of the hydrogen of an acid by a metal that a salt is formed. This replacement may be effected in various ways.

EXPERIMENT 141. Drop small pieces of magnesium ribbon into a small beaker, one-third filled with hydrochloric acid. Add more ribbon when that first used is dissolved, and continue the addition till no more action takes place. Evaporate the solution obtained to a small bulk, and allow it to cool. If the evaporation has been sufficient, a mass of small crystals of magnesium chloride will form on cooling.

Experiment 142. One-third fill a small beaker with dilute nitric acid. Heat this to boiling and drop in small quantities of lead oxide until no more will dissolve. Evaporate and crystals of lead nitrate will be obtained.

Experiment 143. Add crystals of washing-soda to some hydrochloric acid until the portion last added refuses to dissolve. Evaporate to a small bulk, allow to crystallise, and taste one of the crystals formed.

Experiment 144. Place 20 c.c. of potassium hydroxide solution in a beaker and add a few drops of litmus solution. Fill a burette with diluted sulphuric acid and clamp the burette to a retort stand. Place the beaker containing the alkaline solution under the burette so that the tap of the latter is immediately over the middle of the beaker. Run in the acid, 1 c.c. at a time, into the beaker, stirring the liquid in the beaker, after each addition, with a glass rod. When the last portion added causes the colour of the solution to change to red, cease running in the acid, and note the amount which has been added. The 
change of colour may be seen with greater ease if a small white tile be placed under the beaker during the operation. Throw away the contents of the beaker, rinse it out two or three times, and then pipette into it a further 20 c.c. of the alkali. Colour this with litmus solution. Run in 1 c.c. less than the total quantity used in the previous operation, and then let the acid drop in, one drop at a time, stirring after each drop. As soon as the colour change occurs, stop the addition of acid, and read from the burette the exact volume used. This is the volume required to "neutralise" 20 c.c. of the potassium hydroxide solution, and the operation is termed "neutralisation". If a third determination be made as directed in the second case, the reading obtained should agree very closely-within 0.05 c.c.- - with that noted in the second operation.

Pipette 20 c.c. of the alkali into a beaker, and add from the burette the amount of acid which has been found to neutralise it. Evaporate this solution and crystals of potassium sulphate will be obtained.

Experiment 145. Put 20 c.c. of the potassium hydroxide used in the previous experiment into a beaker. Add twice the volume required for neutralisation of the same acid as used before. Again evaporate and crystallise out. If crystals of sufficient size be obtained, compare the shape of those formed in Experiment 144 with that of those now obtained. They will
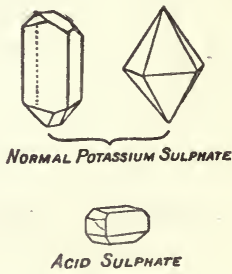

Fig. 102. be found to bear little resemblance to one another. The usual shapes of each kind of crystal are illustrated in Fig. 102. We have thus prepared two substances of 
different form from the same materials, and further investigation of the properties and composition of the two sorts of crystals would show that they are, in fact, two totally different substances. The crystals last obtained are those of a substance called potassium hydrogen sulphate.

Equations :

Magnesium + hydrogen chloride

$$
\text { = magnesium chloride }+ \text { hydrogen. }
$$

Lead oxide + hydrogen nitrate

$$
=\text { lead nitrate }+ \text { hydrogen oxide. }
$$

Sodium carbonate + hydrogen chloride

= sodium chloride + hydrogen oxide + carbon dioxide. Potassium hydroxide + hydrogen sulphate

$=(1)$ potassium sulphate + hydrogen oxide.

$=(2)$ potassium hydrogen sulphate + hydrogen oxide.

217. Potassium hydrogen sulphate belongs to a class of substances termed "acid" salts. This term bears no reference to their reaction, when in solution, with litmus, but is intended to emphasise the presence of some of the hydrogen - the typical element of acidsin the salt, this hydrogen being derived from the acid used in preparing the salt, and being capable of replacement by a metal, just as was the hydrogen already so displaced. Other names for such an acid salt as potassium hydrogen sulphate are potassium acid sulphate and potassium bisulphate. A familiar example of an acid salt is afforded by baking-soda, or sodium bicarbonate.

\section{Exercise for Student.}

Write down other names for baking-soda. Test the reaction upon litmus of solutions of baking-soda and potassium bisulphate.

218. Another class of salts is known, in which a portion of the base remains in combination with the 
salt formed by the neutralisation of an acid by another portion of the base. They are termed "basic" salts. White lead is an example. It consists of lead carbonate combined with lead hydroxide, and is called basic carbonate of lead.

\section{QUESTIONS}

1. Give reasons for considering carbon to be a non-metal.

2. Make a list of all the oxides you know which are soluble in water. What inference may be drawn from the behaviour of the solutions thus formed towards litmus as to the nature of the substance which has entered into combination with oxygen to form the oxide?

3. Why is sodium bicarbonate called an acid salt? Is alum (potassium aluminium sulphate) an acid salt? If not, what would you call it, since its solution turns blue litmus red?

4. Suggest methods for the preparation of the following: sodium chloride; lead chloride; copper nitrate; iron sulphate; potassium nitrate; sodium carbonate.

5. Classify the following substances as binary, ternary, or quaternary compounds: tin oxide; sulphur trioxide; sodium nitrate; potassium bisulphate; copper carbonate; lead iodide; water.

6. Write down the equations for as many of the reactions which occur in Experiments 138, 139, and 140 as you can.

7. Make a list of all the methods of making salts that have been mentioned in the text.

8. If you were supplied with some caustic soda solution and some sulphuric acid, name three substances you could prepare from these materials. Give a full description of the method you would adopt in preparing each of the bodies you mention.

9. 26.4 c.c. of hydrochloric acid are found to neutralise 20 c.c. of a solution of caustic soda. How many c.c. of the alkali will neutralise one litre of the acid?

10. $8 \mathrm{~g}$. of salt are dissolved in $100 \mathrm{~g}$. of water. What weight of salt is contained in $25 \mathrm{~g}$. of the solution ? 


\section{ANSWERS TO QUESTIONS INVOLVING CALCULATIONS}

\section{Chapter I. Page 20.}
1. $1.5 \mathrm{~mm}$.
2. $9 \cdot 9$ in.
3. $69.5 \mathrm{~m}$. ; 42.5 times.
4. 2522 hours; 3668 times.
5. $28 \cdot 8 \mathrm{ft}$.

Chapter II. Page 42.
2. 13 sq. ft.
4. 9 pieces.
5. £5. $4 s .0 d$.
6. $6 s .0 d$.
7. 25,600 .
8. 416,6401 .
9. 1989 tons.
10. $3 \cdot 15$ tons.

Chapter III. Page 62.
1. $106 \frac{2}{3} \mathrm{lb}$; $5 \frac{1}{3} \mathrm{lb}$.
2. 0.75 in.
3. $4 \mathrm{ft}$.
4. $211 \cdot 7$ oz. ; $50,803 \mathrm{~g}$.
5. $6.58 \mathrm{lb}$.
6. $4 \cdot 11$ in.
7. $1,865 \mathrm{~g}$.
9. $30.9 \mathrm{in}$. from centre.

Chapter IV. Page 69.
1. The aluminium cube.
2. $53 \cdot 1 \mathrm{oz}$.
3. $73 \cdot 5$ c.c. $; 787 \cdot 4$ c.c.
4. $0 \cdot 94$.
5. $1,0688 \mathrm{~g}$.
6. $25 \cdot 2 \mathrm{~cm} . \times 12 \cdot 6 \mathrm{~cm} . \times 12 \cdot 6 \mathrm{~cm}$.
7. 16,473 c.c.
8. 2,250 gals.
9. 8,179 c.c. $; 7,500$ g.

Chapter VI. Page 107.

2. $1033 \cdot 6$ g. per sq. cm.; 2,116 lb. per sq. ft.
4. $1 \cdot 024 \mathrm{~g}$. per c.c.
5. $1 \cdot 052$.
6. $24 \cdot 7 \mathrm{~g}$.
7. $2 \cdot 59 ; 1 \cdot 50$.
8. $66 \cdot 8 \mathrm{cu}$. ft.

Chapter VIII. Page 134.

1. $100 \mathrm{ft} .-1 b$. 
Chapter IX. Page 158.

4. $36.9^{\circ} \mathrm{C}$.; $29 \cdot 5^{\circ} \mathrm{R}$.

5. $674 \cdot 6^{\circ} \mathrm{F} . ;-313 \cdot 6^{\circ} \mathrm{F}$.

10. $70 \cdot 7^{\circ} \mathrm{C}$; $-95 \cdot 7^{\circ} \mathrm{C}$.

11. $288 \cdot 3$ c.c. (at $20^{\circ}$ C.).

12. $15 \cdot 681$.; $5 \cdot 861$.

Chapter X. Page 181.

1. $97 \cdot 1^{\circ} \mathrm{F}$,

3. The mercury; the water.

4. $28 \cdot 4^{\circ} \mathrm{C}$.

6. 40,392 cal. ; $40 \cdot 4^{\circ} \mathrm{C}$.

8. $20 \cdot 6 \mathrm{~g}$.

10. $57 \cdot 8^{\circ} \mathrm{C}$.
2. $6.71 \mathrm{Kg}$.

5. $0 \cdot 031$.

9. 25 .

12. 297,108 cal.

Chapter XII. Page 206.

1. $124 \cdot 6 \mathrm{ft}$.

3. 25,929 cal.

5. $18 \cdot 685 \mathrm{Kg}$.
2. $13,275 \cdot 3 \mathrm{Kg} .-\mathrm{m}$.

4. $455 \cdot 6 \mathrm{~m}$.

4. 984 c.c.

Chapter XIII. Page 222.

Chapter XIV. Page 249.

10. $76 \cdot 74 \%$ nitrogen; $23 \cdot 26 \%$ oxygen.

13. $1 \cdot 2075 \mathrm{~g}$.

Chapter XVI. Page 279.

3. $2,708 \mathrm{~g}$.

4. $15 \cdot 88 \mathrm{~g}$.

Chapter XVII. Page 305.

5. $76 \cdot 45 \mathrm{~g}$.

15. $10 \cdot 27 \mathrm{Kg}$.

9. $11 \cdot 22$ c.c. oxygen.

20. 1 cu. ft. steam; 0.5 cu. ft. oxygen.

Chapter XVIII. Page 316.

9. $757 \cdot 5$ c.c.

10. $1.85 \mathrm{~g}$. 


\section{INDEX}

Absolute scale of temperature, 156 ; zero, 156

Absorption of heat, 195

Accuracy of results, 11

Acidic oxides, 260, 309

Acid salts, 315

Acids, action on carbonates, 310, metals, 285, 287, oxides, 310 ; characteristic properties, 310 ; corrosive action of, 310 ; formation of, 309

Actual weight of a body, 95

Air a bad conductor of heat, 186

Air, action on copper, 218, 220; iron, 215; magnesium, 217; phosphorus, 211, 213, 216

Air a mixture, 303

Air, animal life dependent on, 209; aqueous vapour in, 101, 233 ; combustion dependent on, 207 ; composition of, 213,215 , $220,222,241$

Air dissolved in water, 144, 246

Air, part played in chemical change, 219, combustion, 209; presence of carbon dioxide in, 232; specific gravity of, 176

Alkali, derivation of name, 259; characteristic properties of, 311 ; corrosive action of, 311 ; reaction with carbon dioxide, 311

Ammonia in air, 241

Amyl alcohol, 300

Analysis, 262

Aneroid barometer, 100

Apparatus, diagrams of, 10

Aqueous vapour in air, 233

Are, 23

Area, British units, 23; Metric units, 23; determination with balance, 56
Argon, 222 ; isolation of, 218; volume contained in air, 223

Arrestment of balance, 51

Atmosphere, aqueous vapour in, 101, 233

Atmospheric pressure, 96; standard, 107

Atom, 262

Bacteria in air, 241

Baking-soda, 315

Balance, adjustment of, 55; care of, 55; chemical, 50; method of using, 54; use in determining areas, 56

Barium dioxide, action of heat on, 252

Barometer, aneroid, 100; hall, 100 ; measurement of height with, 101 ; simple, 96 ; siphon, 99 ; standard, 100 ; use as weather-gauge, 101

Base, 261

Base, properties of, 311 ; formation of, 309

Basic oxides, 260, 309; salts, 316

Beam of balance, 60

Binary compounds, 308

Body, definition of, 43

Body temperature, mäintenance of, 227

Boiler, cause of bursting of, 175

Boiling-point, effect of pressure on, 144 ; determination of, 143

Boiling-point of solutions, 178

Bolthead, 230

Boyle's experiment, 102; Law, 104, application of, 106

Brin's process, 252

British System, relation of units, 53 ; unit of length, 2, power, 121, work, 119 
Bromine, care in handling, 74

Bunsen cell, 283

Burette, 39; testing graduation of, 59 ; use of, 313

Calcium, action on water, 281

Calcium carbonate, 225; chloride, as drying agent, 229, 234, 286, 288,296 ; oxide, 225

Calipers, use of, 16,17

Calorie, 161; relation to gramcentimetre, 201

Calorimeter, 173

Candle, constituents of, 224

Capacity, units of, 34

Carbon, combustion in oxygen, 256

Carbon dioxide, action on water, 258; dissolved in water, 243, 246 ; composition of, 263 ; specific gravity of, 177; food of plants, 242; formation from elements, 256; presence in air of, 232,242 ; in air, sources of, 253 ; in expired air, 233 ; product of combustion, 22, decay, 244 , plant life, 243 , respiration, 226

Carbonic acid, 256, 258

Carbon present in food, 227

Catalysis, 255

Caustic potash, absorbent for carbon dioxide, 229, 246

Caustic soda, 259

Celsius scale of temperature, 147

Centimetre, 3

Centre of gravity, 109; of plane figure, 109

Centrifugal force, 47

Chalk, 225; composition of, 302

Change of colour produced by heat, 131; state produced by heat, 130

Charles's Law, 154, 156

Chemical action, signs of, 303; change, 133, 217

Chemical change, produced by heat, 131

Chemical classification, 306 ; combination, 217,262 , heat produced during, 218; decomposition, 262; energy, 261, 262; equations, 217, 262; formulae, 262; nomenclature, 309 ; symbols, 262

Circle, area by squared paper, 31 ; ratio of circumference to diameter, 15

Classification, function of, 81 ; of compounds, 308; of elements, 307

Clinical thermometer, 149

Clothes, choice of, 197

Coal, energy stored in, 202

Cobalt chloride, use of, 235

Cohesion, 73, 77

Collection of gases by downward displacement of air, 289

Combustion, dependent on air, 207 ; increase of weight during, 227

Compound, 224; distinction from mixture, 302

Compounds, binary, 308; classification of, 308; quaternary, 309; ternary, 309

Condenser, Liebig, 269; worm, 269

Conduction of heat, 184, 185

Conductors of heat, good and bad, 185

Cone, volume of, 37

Constant Composition, Law of, 301

Convection currents in gases, 189 ; in liquids, 187

Copper, action of air on, 218; sulphate, anhydrous, 289

Cotton-wool, poor heat conductor, 169,171

$\mathrm{CO}_{2}$, meaning of formula, 262

Counterpoising, method of, 57

Critical pressure, 82 ; state, 82 ; temperature, 82

Crystallisation, 271; heat evolved during, 273

Crystals, 314; growth of, 271

Cube, 33

Cubical expansion by heat, 126

Cubic centimetre, comparison with cubic inch, 34

Cubic inch, relation to pint, 34

Curved lines, measurement of, 13, 15

Curve plotting, 236

Cylinder, volume of, 37 
Dalton's Law, 154, 156

Daniell cell, 283

Decametre, 3

Decantation, 277

Decay; 244

Decimal system of numeration, 3

Decimetre, 3

Definitions, function of, 81

Deflagrating spoon, 210

Degradation of energy, 202, 261

Density, definition of, 64

Density, distinction from specific gravity, 65; flask, 67 ; of a gas, 63 ; of liquids, 66

Diffusion, 78; of gases, 74, in atmosphere, 242 ; of liquids, 75 ; of solids, 76 ; through porous media, 79

Distillation of water, 266

Distilled water, action on glass, 267 ; properties of, 267

Dividers, use of, 13

Downward displacement, collection by, 289

Dry steam, 172

Dust in air, 241

Ebullition, 144

Electrical energy, transformation of, 285

Electrode, 285

Electrolysis, 285

Electrolyte, 285

Element, 224

Elements, classification of, 307

Endothermic reactions, 261

Energy, 121; chemical, 261; convertibility of, 124, 201, 261, 290 ; degradation of, 202,261 ; examples of kinetic, 123, potential, 122 ; forms of, 124 ; indestructibility of, 123; kinetic, 122 ; potential, 122

Entry of experiments, 8, 12

Equilibrium, neutral, 115; relation to position of centre of gravity, 115; stable, 114; unstable, 114

Ether, heat absorbed during vaporisation, 173; nature of, 191 ; precautions in using, 173; wave motion in, 192
Ether-waves, magnitude of, 192

Eudiometer, 299

Evaporation, 144, 235; to dryness, 265

Exothermic reactions, 261

Expansion by heat, cubical, 126; linear, 125; of gases, 128; of liquids, 127

Explosion, nature of, 290

Fahrenheit scale of temperature, 147

Ferric oxide, 248

Filter paper, method of folding, 275

Filtering materials, 275

Filtration, 275

Fire as ventilating agent, 208

Fish, respiration of, 248, 249

Fletcher burner, 281

Fluid pressure, calculation of, 85 ; equality in all directions, 87; experimental inferences, 86 ; nature of, 83

Fluids, 73

Foot-pound, 119

Force, definition of, 44; due to expansion by heat, 133; of gravity, 44, 45, measurement of, 45 , relation to mass, 46

Formula, chemical, 262

Freezing mixtures, 180; use of, 166

Freezing-point, effect of dissolved substances on, 180

Fuels, constituents of, 226; products of combustion of, 226

Fulcrum, 49

Fusion, heat absorbed during, 165

Gaseous Combination, Law of, 302

Gases, action of heat on, 204; diffusion of, 74; dissolved in water, 244,247 , importance of, 243,248 ; expansion by heat, 128; properties of, 73 ; relation of volume to temperature, 152; specific gravity of, 177

Gay-Lussac's Law, 302

Glauber's salt, 273

H. D. S. 
Graduated cylinder, 40; vessels, method of reading, 40

Graduation of thermometer, 141

Gram-centimetre, relation to calorie, 201

Gravesande's ball and ring, 126

Gravitation, 44

Gravity, centre of. 109

Gravity, universal action of, 44

Grinding glass surfaces, 84

Heat absorbed during liquefaction, $165,169,180$; solution, 234 ; vaporisation, 167

Heat, absorption of, 195; action on gases, 204

Heat a form of energy, 125, 133, 200,204 ; a mode of motion, 204; and temperature, distinction between, 159; as a quantity, calculations involving, 162

Heat, change of colour by, 131, state by, 130; chemical change produced by, 131

Heat evolved during combination, 218, condensation, 172, solidification, 166

Heat, expansion by, 125; interchange of, 148; latent, 166; mechanical equivalent of, 201

Heat rays, comparison with light rays, 194

Heat, reflection of, 196

Hectare, 23

Hectometre, 3

Hexagon, area of, 27

Hofmann's voltameter, 283

Horse-power, 121

Hot water system of heating, 188

Hydraulic press, 87

Hydrochloric acid, action of heat on, 312 ; care in handling, 75 ; for gas preparation, 285

Hydrogen, action on copper oxide, 297, iron oxide, 298; combustion in air, 282, 284; contained in acids, 313,315 ; explosion with oxygen, 291,300 ; explosive mixture with air, 286,290 ; laboratory prepara- tion of, 285; lighter than air, 282,286

Hydrogen peroxide in air, 241

Hydrogen, preparation from steam, 282 , water, 280,283 ; present in air, 241, foods, 227 ; a product of combustion, 288 ; temperature of flame of, 288

Hydrometer, 92; convenient ranges for, 93; method of reading, 93; nature of graduation of, 92 ; testing accuracy of, 94; use of, 93

Hygrometer, wet and dry bulb, 273

Hygroscopic substances, 234

Hypothesis, function of, 76

Ice and salt as freezing mixture, 166,180 ; and water, relation between volumes of, 174 ; contraction during fusion, 174

Indestructibility of Energy, 123; of Matter, 230, 231

Ingenhaus's apparatus, 184

Invisible ink, 235

Iron, action of air on, 215 ; action on steam, 281 ; black oxide of, 260,283 ; combustion in oxygen, 259; reduced, 221

Irregular figure, area by squared paper, 28; rectilineal figure, area of, 27; solids, volume by displacement of liquids, 40,41

Kilogram-metre, 119

Kilogram, relation to pound, 53, unit of length, 53 ; standard, 53

Kilometre, 3

Kilowatt, 121

Kinetic energy, 123; examples of, 123 ; transformation from potential, 123; transformation into heat, 124

Kinetic Hypothesis, 77, 191; recapitulation, 202; applied to change of state, 203, expansion by heat, 203, pressure changes, 205

Kipp's generator, 177, 294

Knife-edges, 51 
Lactometer, 94

Lampblack as absorbent of heat, 196

Lamp resistance, use of, 283

Latent heat, 166

Latent heat of fusion, 166, determination of, 169 ; of vaporisation, 168, determination of, 171

Latent heats, table of, 168

Lavoisier and oxygen, 257

Lavoisier, volume of oxygen in air, 250

Law of Constant Composition, 301; of Gaseous Combination, 302

Lead carbonate, basic, 316 ; nitrate, preparation of, 313 ; oxide, action of heat on, 253

Length, British standard unit, 2; Metric standard unit, 3

Lengths, estimation of small, 7

Liebig condenser, 269

Light, combination accompanied by, 218

Light rays similar to heat rays, 194

Lime, 225

Lime-water as test for carbon dioxide, 223

Linear expansion by heat, 125

Liquefaction, heat a bsorbed during, 165

Liquid air, 304

Liquids, diffusion of, 75 ; expansion by heat, 127; need for lateral support, 73; properties of, 71

Litre, 34

Lowering of freezing-point by dissolved matter, 180

Magnesium, action of air on, 217; action on steam, 281

Magnesium chloride, preparation of, 313 ; nitride, 218 ; oxide, 218

Manganese dioxide, action of heat on, 253; as catalytic agent, 255

Manipulation of glass tubing, 138

Mariotte's Law, 105

Mass, constancy of, 44; definition of, 44 ; equality of, 47 ; re- lation to weight, 46 ; standard units of, 54

Matter, definition of, 43

Maximum thermometer, 151

Measure, unit, 1

Measuring vessels, 39

Mechanical equivalent of heat, 201

Meniscus, 41

Mercury, action of heat on, 251

Metals, good conductors of heat, 185; general properties of, 307

Metaphosphoric acid, 257, 259

Metre, multiples of, 3; standard, 3 ; submultiples of, 3

Metric System, advantages of, 4, $24,35,53$; conversion of units, 4 ; measures of area, 23 , length, 3 , volume, 33 ; origin of, 3 ; prefixes used in, 4; relation to British, 9, 10; relation between units, 34, 53; unit of heat, 161, power, 121, work, 119

Millimetre, 3

Minimum thermometer, 150

Miscibility of liquids, 278

Mixture distinguished from compound, 302

Molecule, 77

Molecules, freedom of motion of, 77 ; kinds of, 262; relative size of, 79

Moment, 50

Natural waters, 264

Neutral equilibrium, 115

Neutralisation, 310,313

Neutral oxide, 261

Newton, Sir Isaac, 44

Nicholson and Carlisle, 285

Nitre, action of heat on, 253

Nitric acid in air, 241

Nitrogen, action on magnesium, 218; boiling-point of, 304 ; dissolved in water, 246 ; separation from air, 211, 213, 215, 220; volume in air, 222; weight in air, 222

Non-metals, properties of, 307

Organic compounds, nature of, 308

Ounce, use as fluid measure, 40

Oxidation, 221 
Oxidation attended by gain in weight, 229; of food, 227

Oxides, acidic, 309; basic, 309; neutral, 261

Oxy-acids, 312

Oxygen, action on iron, 259, phosphorus, 230, 257, sodium, 257; as acid producer, 257, 258 ; as supporter of combus. tion, 255, 284; boiling-point of, 304 ; derivation of name, 257 ; dissolved in water, 246,248 ; from air, 250, 252, barium peroxide, 252, red lead, 253, liquid air, 304, manganese dioxide, 253, mercuric oxide, 131, oxygen mixture, 254, potassium chlorate, 253 , potassium nitrate, 253, water, 283 ; present in air, 215, 242; waste product of plant life, 242

Ozone, presence in air, 241

Parallelogram, area of, 25

Pascal, experiments on atmospheric pressure, 97

Peroxide, 261

Phenomenon, 76

Phlogiston, 264

Phosphorus, combustion of, 210 , 230, 257; slow oxidation of, 216

Phosphorus pentoxide, 216, 224, 234,257

Phosphorus, precautions in using, 210

Physical change, 132

$\pi$, determination of value of, 15,31

Pint measure, graduation of, 40

Pint, relation to cubic inch, 34

Pipette, 39 ; method of using, 57

Pipette, testing accuracy of, 57

Plants, absorption of carbon dioxide by, 242; excretion of oxygen by, 242

Plasticine, use of, 35

Platinum jet, 256, 288

Potassium acid sulphate, preparation of, 314

Potassium, action on water, 281

Potassium bisulphate, 314; carbonate, 229 ; chlorate, action of heat on, 253, crystallisation of,
274 , solubility of, 274 ; chloride, 253; hydrogen sulphate, 314; hydroxide, action on carbon dioxide, 229; nitrite, 253 ; nitrate, action of heat on, 253 ; sulphate, preparation of, 313

Potential energy, 122; examples of, 122; transformation into kinetic, 123

Pound, distinction between weight and mass, 54; relation to kilogram, 53; standard, 52

Power, 121; British unit of, 121 ; Metric unit of, 121

Precipitate, 225

Pressure, atmospheric, 96; critical, 82 ; measurement of, 83 ; standard atmospheric, 107

Principle of Archimedes, 90; applied to gases, 94 ; verification of, 89 ; use of, 90,91

Principle of floating bodies, 92

Principle of Moments, 50

Purification of water, 266, 268

Pyknometer, 67

Pyramid, volume of, 37

Pyrogallic acid, use of, 246

Quaternary compound, 309

Radiant heat, 194

Radiation, 194

Radiation from hot solid, 193

Radium, energy stored in, 202

Raising of boiling-point by dissolved substance, 178

Ramsay burner, 125

Ramsay, Sir William, 218

Reading graduated vessels, 40

Réaumur's thermometer scale, 147

Rectangle, area of, 24

Rectangular parallelopiped, 36; prism, volume of, 36 ; slab, volume of, 36

Red lead, action of heat on, 253

Reduced iron, 221

Reducing agent, 305

Reflection of heat rays, 196

Regular figures, areas of, 24; solids, formulae for volumes of, 38 , volumes of, 36 
Relative density, definition of, 65

Respiration of fish, 248

Respired air, carbon dioxide in, 232

Results, degree of accuracy of, 11; method of checking, 7

Retort, tubulated, 266

Reversible reactions, 298

Rusting, conditions of, 247, 248; oxygen absorbed in, 215; part played by carbon dioxide, 248

Rust, nature of, 217,248

Rutherford's thermometers, 150 , 151

Saltpetre, action of heat on, 253

Salts, acid, 315 ; basic, 316 ; formation of, 310,313 ; reactions of, 311,312

Sand-bath, 269

Saturated solution, 272; preparation of, 271

Scale of temperature, absolute, 156; Fahrenheit, 147; Centigrade, 147; Réaumur, 147

Scales, 5

Scale, use of, 6

Silica, 309

Silicic acid, 309

Silicon dioxide, 309

Siphon barometer, 99

Six's thermometer, 151

Snow, poor conductor of heat, 186

Sodium, action of water on, 280

Sodium bicarbonate, 315 ; chloride, preparation, 313

Sodium, colour of flame of, 281 ; combustion in oxygen, 257; hydroxide, 258, 259, 281; hyposulphite, 233; peroxide, 258; precautions in using, 257 ; sulphate, 273 ; thiosulphate, 233

Solidification, heat evolved during, 166,273

Solids, diffusion of, 76 ; properties of, 70,71

Solubility, effect of temperature on, 271; method of testing, 278

Solubility of liquids, 278

Soluble and insoluble substances, method of separation, 275

Solution, heat absorbed during, 180,233 ; preparation of satu- rated, 271; removal of gases from, 245; saturated, 272; supersaturated, 272

Solutions, boiling-point of, 178; freezing-point of, 180

Soot in air, 241

Specific gravities, table of, 68 ; gravity, definition of, 65, distinction from density, 65

Specific gravity of irregular solids, 90 ; liquids, 91 ; milk, 94

Specific heat, 162; calculations involving, 163

Specific heats, table of, 163

Sphere, volume of, 37

Spring-balance, $\mathbf{4 5}$

Square centimetre, relation to square inch, 27 ; decimetre, com parison with square foot, 22

Squared paper, testing accuracy of, 31 ; use in curve plotting, 236; use in estimating areas, 28

Stability, degrees of, 114; relation to the centre of gravity, 113

Stable equilibrium, 114

Standard units, 2

States of matter, intermediate forms, 81

Steam, action on iron, 281, magnesium, 281; composition of, 300 ; constant temperature of, 168,179 ; pressure when confined, 175

Steam-trap, 171

Steam, volume compared with that of water, 61

Steelyard, method of graduation, 61 ; principle of, 61 ; weights for, 61

Still, 270

Straight lines, measurement of, 6

Substance, definition of, 43

Sulphur, combustion in oxygen, 256

Sulphur dioxide, action on water, 258; preparation of, 256 ; presence in air, 241

Sulphuretted hydrogen, presence in air, 241

Sulphuric acid, 258; use as drying agent, 240,296

Sulphurous acid, 256, 258 
Sulphur trioxide, 256

Supersaturated solution, 272, 273

Symbol, 262

Synthesis, 262

Table of latent heats, 168; specific gravities, 68; specific heats, 163

Tape-measure, disadvantages of, 5 Tea-cosy, reason for use of, 186

Temperature, absolute scale of, 156; distinction from heat, 159; critical, 82; definition of, 148; maintenance of body, 227

Ternary compounds, 309

Thermal capacity, 162

Thermometer, 136; Clinical, 149; construction of, 137; determination of fixed points on, 146; graduation of, 141 ; maximum, 151; method of filling, 139, sealing, 140; minimum, 150,151 ; readings, change of scale of, 147; rise of zero, 142; scales used for, 147; Six's, 151

Thermometers, checking laboratory, 142; Rutherford's, 150, 151

Thermos flask, 186

Torricelli, 98

Torricellian vacuum, 99

Transference of heat, conditions of, 148

Transformations of energy, 123, $261,285,290$

Transmission of heat through fluids, 187; solids, 183; space, 192

Triangle, area of, 25

Unit of heat, 161

Unit measure, 1

Units, standard, 2

Unstable equilibrium, 114

Upthrust, 85, 86

U-tube barometer, 99; eudiometer, 299

Vacuum a bad conductor of heat, 186

Vaporisation, effect of air-currents on, 173; heat absorbed during, 167,237 ; latent heat of, 168

Vapour, definition of, 106

Ventilation of coal-mines, 190; of living-room, 190

Vernier, principle of, 19; simple, 18

Vision, stimulation by ether waves, 192

Volume, British units, 33; Metric units, 33; relation between weight and, 63

Volumes of gases, calculations involving, 155

Walls, method of strengthening, 133

Water, a bad conductor of heat, 185; a compound, 303 ; action on calcium, 281, copper, 281, glass, 267, iron, 247, 281, magnesium, 281, potassium, 281, sodium, 280; analysis of, 283; and ice, relation between vol. umes of, 174; and steam, relation between volumes of, 175; as a standard substance, 65; -bath, 265; behaviour when cooled, 293, heated, 143; characteristic properties of, 291, 294; chemical test for, 289 ; composition by volume, 285, 298, 299, weight, 294, 298; distinction from its elements, 224; dissolved gases in, 144; distillation of, 266 ; -equivalent, 164; expansion during solidification, 174; high specific heat of, 161 ; anomalous expansion of, 292; importance of dissolved gases in, 243 ; maximum density of, 293 ; -pipes, cause of bursting, 174; product of combustion, 223,288 , of neutralisation, 310 , of respiration, 226; solids dissolved in, 265; solubility of liquids in, 278; synthesis of, 294; vapour in air, 233, measurement of, 239, sources of, 235

Weighing in a vacuum, 95 in water, 89 
Weighing-machine, 60

Weight a measure of mass, 48

Weight, British standard unit, 52 ; causes of variation of, 47 ; definition of, 46; Metric standard unit of, 53; actual, 95 ; relation between mass and, 46 , volume and, 63

Weights, British system, 53; equality of, 47; Metric table of, 53 ; usual contents of box of, 54

Wet and dry bulb hygrometer, 237

White lead, 316
Work, British unit of, 119

Work done against force, 120; by force, 119

Work, measurement of, 118; Metric unit of, 119

Worm-condenser, 269

Yard, legal, 2; multiples of, 2; submultiples of, 2 .

Zero, use before fractions of units, 12

Zinc chloride, preparation of, 286

CAMBRIDGE : PRINTED BY JOHN CLAY, M.A. AT THE UNIVERSITY PRESS 



\section{THE CAMBRIDGE TECHNICAL SERIES \\ General Editor: P. ABBOTT, B.A., \\ HEAD OF THE MATHEMATICAL DEPARTMENT, THE POLYTECHNIC, REGENT STREET, LONDON}

\section{Now ready}

A Manual of Mechanical Drawing. By J. H. DaI.Es, C.E. 3 s net; or in two parts, 2 s net each.

Electrical Engineering. Vol. I. Introductory. By T. C. Barllie, D.Sc., A.M.I.E.E. 5s net.

Architectural and Building Construction Plates. By

Walter R. Jaggard, F.R.I.B.A. PART I. 30 Plates. $6 \mathrm{~s}$ net.

PART II. 30 Plates. 6s net.

Domestic Science. Part I. By C. W. Hale.

\section{In the press}

Applied Mechanics. By Ewart S. ANdrews, B.Sc.

Alternating Currents. By W. H. N. JAmes, A.R.C.Sc. (Lond.), A.M.I.E.E.

History of English Building Construction. By C. F. INNOCENT.

Sculpture in relation to Architecture. By T. P. Bennet, A.R.I.B.A.

Naval Architecture. By J. E. Steele, B.Sc., A.M.I.N.A.

\section{In preparation}

Architectural Building Construction. In three volumes. By W. R. JAGGard and F. E. DruRy.

Electrical Engineering. Vol. II. By T. C. Baillie, D.Sc.

Domestic Science. Part II. By C. W. Hale.

Automobile Engineering. By A. Graham Clark, M.I.A.E., A.M.I.Mech.E.

Electro-Technical Measurements. By A. E. Moore. A.M.I.E.E.

Chemistry and Technology of Oils and Fats. By F. E. Weston, B.Sc., and P. J. Fryer.

Paper, its Uses and Testing. By Sheldon Leicester.

Mining Geology. By Professor G. Knox and S. RatcliffeE.LLIS.

Textile Calculations-Materials, Yarns and Fabrics. By A. Mrtchell Bell.

Laboratory Note Book for Applied Mechanics and Heat Engines. By F. Boulden, B.Sc.

Elements of Applied Optics. By W. R. Bower, B.Sc.

Physics for Engineers. By J. PALEy Yorke.

Electric Installations. By C. W. HiLl, A.M.I.C.E., M.I.E.E. Accounting. By John B. Wardhaugh, C.A.

Chemistry for Textile Students. By BARker North, A.R.C.S., and Norman Bland, B.Sc.

Chemistry of Dyeing. By Dr L. Lloyd and Morris Fort, B.Sc. Dyeing and Cleaning. By FrANKLIN W. WALKER. 


\section{THE CAMBRIDGE UNIVERSITY PRESS}

Domestic Economy in Theory and Practice. A Text book for Teachers and Students in Training, by MARION GREENwOoD BIDDER, of Newnham and Girton Colleges, and FloRencE BADDELEY, Organizing Secretary of the Gloucestershire School of Cookery and Domestic Economy. Crown 8vo. With several diagrams. $4 \mathrm{~s} 6 \mathrm{~d}$.

"It supplies an intelligent exposition of such scientific knowledge as is required to the proper understanding of the subject, and a detailed explanation of what should be done in practical teaching... The result is a volume which stands in a class by itself as a valuable exposition of domestic economy in theory and practice."-Educational News

Experimental Science. By S. E. Brown, M.A. (Cantab.), B.A., B.Sc. (Lond.), Headmaster of Liverpool Collegiate School. Crown 8vo. In 2 parts. Part I. Physics. 3s 6d. Part II. Chemistry. 2s. The two parts may be obtained bound in one volume, or may be purchased together for 5s. Part I may also be obtained in four sections: I, Measurement; II, Hydrostatics; III, Mechanics; IV, Heat. Is each.

"The nearest approach to the ideal laboratory and taxt-book that we have yet seen."-Secondary School Journal.

Outlines of Chemistry. With practical work. By H. J. H. Fenton, M.A., Sc.D., F.R.S. Part I. Second (enlarged) edition. Demy 8vo. 9s net.

Physical Chemistry for Schools. By H. J. H. Fenton, Sc.D. [In preparation Inorganic Chemistry. By E. I. LewIS, B.A. (Cantab.), B.Sc. (Lond.), Headmaster of the Methodist College, Belfast. Second edition. Demy 8 vo. In one volume. $5 \mathrm{~s}$. Or in two parts, 3s each.

Notes on Elementary Inorganic Chemistry. By F. H. JEFFERY, M.A. Demy 8vo. 2s $6 \mathrm{~d}$ net. With blank pages interleaved. 3 s net.

A Manual of School Hygiene. Written for the guidance of Teachers in Day-schools by EDWARD W. HopE; M.D., EDGAR A. Browne, F.R.C.S.E., and C.S. Sherrington, M.D., F.R.S. New and revised edition with six additional chapters on Physiology. Crown 8vo. With several diagrams. 4s $6 \mathrm{~d}$.

Air-Currents and the Laws of Ventilation. By W. N. SHaw, Sc.D., F.R.S. Demy 8 vo. 3s net.

House-Flies and how they spread disease. By C. G. HewitT, D.Sc. Royal I6mo. With 20 illustrations. Cloth, Is net; leather, 2s 6d net. Cambridge Manuals Series.

The Story of a Loaf of Bread. By T. B. Wood, M.A. Royal I6mo. With $\mathrm{I} 7$ illustrations. Cloth, is net; leather, $2 \mathrm{~s} 6 \mathrm{~d}$ net. Cambridge Manuals Series.

\section{Cambridge University Press}

C. F. Clay, Manager: Fetter Lane, London 



\section{DAY USE \\ RETURN TO DESK FROM WHICH BORROWED LOAN DEPT.}

This book is due on the last date stamped below, or on the date to which renewed.

Renewed books are subject to immediate recall.

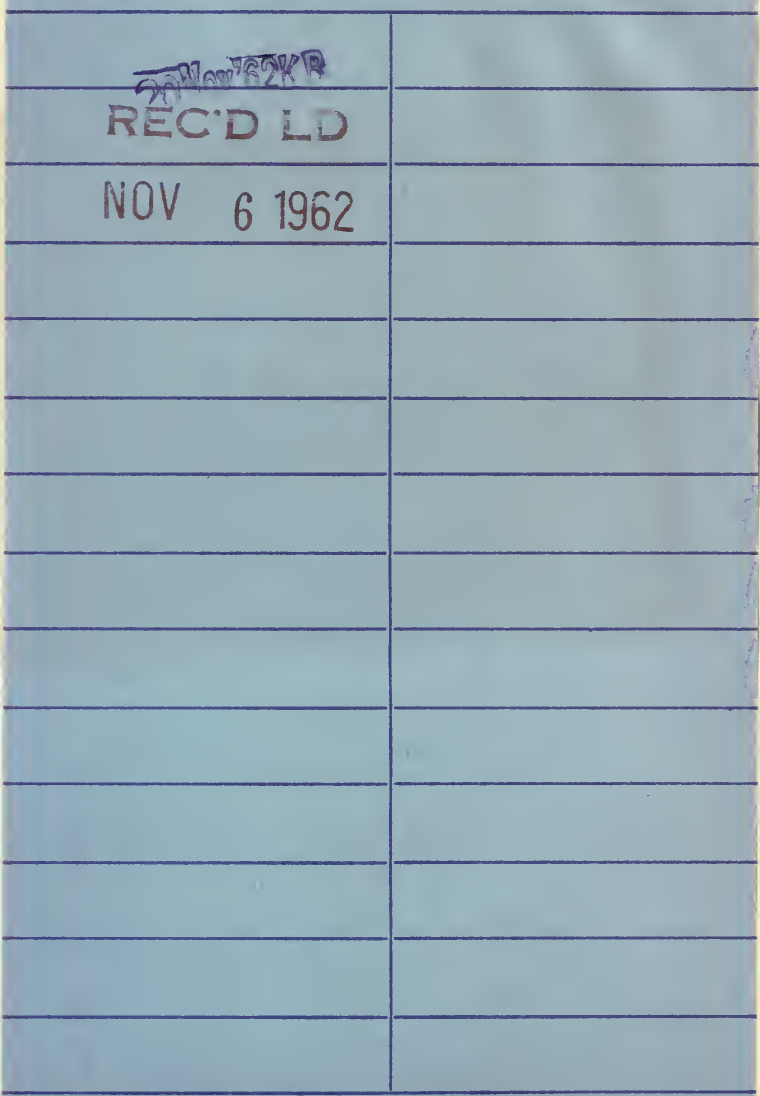




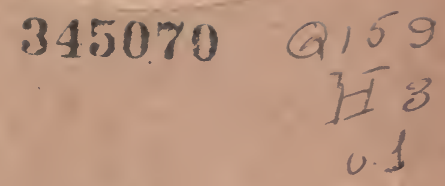

Hale

UNIVERSITY OF CALIFORNIA LIBRARY 


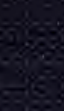

